\title{
Die Totalsynthese von Chlorotonil A
}

\author{
Von der Naturwissenschaftlichen Fakultät \\ der Gottfried Wilhelm Leibniz Universität Hannover
}

zur Erlangung des Grades

Doktor der Naturwissenschaften

- Dr. rer. nat. -

genehmigte Dissertation

von

Diplom-Chemikerin Nicola Rahn

geboren am 10.08.1979

in Hildesheim 
Hiermit versichere ich an Eides statt, die vorliegende Dissertation selbständig durchgeführt und keine unerlaubte Hilfe in Anspruch genommen zu haben. Die aus fremden Quellen übernommenen Gedanken sind als solche kenntlich gemacht.

Diese Arbeit wurde weder zuvor veröffentlicht, als Dissertation, Diplomarbeit oder ähnliche Prüfungsleistung verwendet noch an einer anderen Fakultät eingereicht.

Hannover, im April 2007

Referent: $\quad$ Prof. Dr. Markus Kalesse

Koreferent: Prof. Dr. Andreas Kirschning

Tag der Prüfung: 18.05.2007 


\section{Kurzfassung}

Rahn, Nicola

\section{Die Totalsynthese von Chlorotonil A}

Schlagworte: Totalsynthesen, Naturstoffe, Chlorotonil A

Chlorotonil A ist ein tricyclischer Naturstoff, der sich aus einem Decalin-System und einem 14-gliedrigen Macrolacton zusammensetzt. Er wurde von Mitarbeitern des HZI, K. Gerth und R. Jansen, im Jahre 2004 aus dem Myxobakterium Sorangium cellulosum isoliert und weist antibiotische Eigenschaften auf. Im Rahmen dieser Dissertation konnte die erste Totalsynthese von Chlorotonil A erfolgreich abgeschlossen werden. Die hier beschriebene Synthese umfasst 21 lineare Stufen bei einer Gesamtausbeute von 1.5\%. Der Schlüsselschritt ist eine Lewis-Säure katalysierte Kaskadenreaktion, bei der eine PMB-Schutzgruppe entfernt wird sowie eine Macrolactonisierung und Diels-Alder Reaktion stattfinden. Das Resultat ist ein Decalin-System mit vier tertiären Stereozentren, die auf diese Weise effizient und selektiv generiert werden. Der Aufbau des Z,E-Diens von Chlorotonil A erfolgt durch eine vinyloge Still-Gennari Reaktion und kann damit in einem Schritt dargestellt werden. Die Tricarbonyl-Struktureinheit wird durch Addition von Ethyl-2methylacetoacetat an einen Methylester eingeführt. Die dabei entstehenden Diastereomere können nach Lewis-Säure katalysierter Macrolactonisierung durch die Epimerisierung des Stereozentrums an $\mathrm{C}(2)$ in eine diastereomerenreine Verbindung, das gewünschte Macrolacton, überführt werden. Die anschließende selektive Chlorierung ergibt das Zielmolekül. 


\begin{abstract}
Rahn, Nicola

\section{The Total Synthesis of Chlorotonil A}

Keywords: Total synthesis, natural products, chlorotonil A

Chlorotonil A is a tricyclic natural product consisting of a decalin system and a 14membered macrolactone. It was isolated by coworkers of the $\mathrm{HZI}, \mathrm{K}$. Gerth and R. Jansen, in 2004 from myxobacteria Sorangium cellulosum and shows antibiotic properties. The first total synthesis of chlorotonil $A$ is described. This convergent synthesis has 21 linear steps and $1.5 \%$ overall yield. The key step is a Lewis-acid catalyzed domino reaction of PMB-deprotection, lactonization and Diels-Alder reaction that allows the construction of the decalin system and its four tertiary stereocentres at a time. Moreover the E/Z-diene can be introduced via a vinylogous Still-Gennari reaction in one step. The tricarbonyl system is generated by the addition of ethyl-2-methylacetoacetate to a methyl ester. The resulting diastereomers can be transformed into a single product, the desired macrolactone, after Lewis-acid catalyzed macrolactonization and through epimerization of the $\mathrm{C}(2)$ stereocenter. The selective chlorination of the macrolactone finally gives the target molecule.
\end{abstract}




\section{Danksagung}

Die vorliegende Arbeit wurde im Zeitraum vom Juli 2004 bis zum April 2007 unter der Leitung von Prof. Dr. Markus Kalesse am Institut für Organische Chemie an der Universität Hannover angefertigt.

Für die Überlassung des Themas, die Betreuung während der Doktorarbeit und die ständig vorhandene Diskussionsbereitschaft möchte ich mich bei meinem Doktorvater Prof. Dr. Markus Kalesse herzlich bedanken.

Prof. Dr. Andreas Kirschning danke ich für die Übernahme des Koreferates und die gute Zusammenarbeit zwischen den Arbeitskreisen Kirschning und Kalesse.

Meinen Laborkollegen Romy Schäckel, Svetlana Zimmermann und Jorma Hassfeld danke ich für die angenehme Arbeitsatmosphäre im Labor sowie dem ganzen Arbeitskreis Kalesse und Boysen für die schöne Zeit inner- und außerhalb des Labors.

Den Mitarbeitern der Spektroskopie, Dr. Albert, Monika Rettstadt und Dagmar Körtje danke ich für ihre Hilfsbereitschaft.

Mike Boysen, Gunnar Ehrlich, Romy Schäckel, Claudia Noll, Serkan Simsek und Dominic Jansen danke ich für das Korrekturlesen der Arbeit.

Mein ganz besonderer Dank gilt meinem Mann Kjell Patrick Rahn für seine Unterstützung und sein Verständnis für die Überstunden im Labor während der letzten Jahre. 


\section{Inhaltsverzeichnis}

1. Einleitung 1

1.1 Morphologie der Myxobakterien 1

1.2 Myxobakterien als Produzenten biologisch aktiver Sekundärmetabolite 4

$\begin{array}{lll}1.3 & \text { Die Bedeutung der Naturstoffsynthese } & 7\end{array}$

$\begin{array}{ll}\text { 2. Aufgabenstellung } & 8\end{array}$

3. Synthetischer Teil 9

3.1 Retrosynthetische Analyse von Chlorotonil A 9

3.2 Synthese des Vinylboronsäureesters 12

3.3 Synthese des Dibromolefins 14

3.4 Suzuki Kupplung von Vinylboronsäureester und Dibromolefin 16

3.5 Intramolekulare Diels-Alder Reaktion 18

3.6 Aufbau der Dien-Seitenkette 28

3.7 Aufbau der Triketon-Seitenkette 33

3.8 Macrolactonisierung und abschließende Halogenierung 39

3.9 Dehalogenierung von Chlorotonil A 42

3.10 Zusammenfassung und Ausblick $\quad 44$

4. Experimenteller Teil 46

4.1 Allgemeine Bemerkungen 46

$\begin{array}{lll}4.2 & \text { Beschreibung der Versuche } & 48\end{array}$

$\begin{array}{ll}\text { 5. Literaturverzeichnis } & 103\end{array}$

\section{Anhang}




\section{Abkürzungsverzeichnis}

Ac

Acetyl

$\mathrm{Bn}$

Benzyl

$\mathrm{Bu}$

Butyl

DDQ

2,3-Dichlor-5,6-dicyano-1,4-benzochinon

DiBAl-H

Diisobutylaluminiumhydrid

DIPEA

Diisopropylethylamin

DMAP

$N, N-4$-Dimethylaminopyridin

DMF

$N, N$ - Dimethylformamid

DMSO

Dimethylsulfoxid

eq

Equivalent

Et

Ethyl

EtOAc

Essigsäureethylester

$\mathrm{HZI}$

Helmholtz Zentrum für Infektionsforschung

HPLC

High performance liquid chromatography

HRMS

High resolution mass spectroscopy

IMDA

Intramolekulare Diels-Alder Reaktion

$i \mathrm{Pr}$

Isopropyl

KHMDS

Kaliumhexamethyldisilazid

$\mathrm{Me}$

Methyl

MTBE

tert-Butylmethylether

NMO

N-Methylmorpholin-N-oxid

NMR

Nuclear magnetic resonance, Kernresonanz

$\mathrm{Ph}$

Phenyl

PMB

para-Methoxybenzyl

RT

TADA

Raumtemperatur

TBDPS

Transannulare Diels-Alder Reaktion

TBS

tert-Butyldiphenylsilyl

THF

tert-Butyldimethylsilyl

TMS

Tetrahydrofuran

Trimethylsilyl

TPAP

Tetra- $n$-propyl-ammoniumperruthenat 



\section{Einleitung}

Als Quelle für neue biologisch aktive Verbindungen dienen neben Pflanzen vor allem Mikroorganismen. ${ }^{[1]}$ Viele der Stoffe mikrobiellen Ursprungs finden unter anderem Anwendung als Therapeutika in der Human- und Veterinärmedizin sowie in der Landwirtschaft als Futtermittelzusätze oder für den Pflanzenschutz. Die Produktion von Sekundärmetaboliten durch Mikroorganismen ist damit nicht nur von akademischem Interesse, sondern besitzt auch große wirtschaftliche Bedeutung. Die heute etwa 10.000 bekannten biologisch aktiven Verbindungen aus Mikroorganismen wurden aus einer verhältnismäßig kleinen Gruppe von Bakterien und Pilzen isoliert. Eine Möglichkeit, neue Substanzen zu finden, ist das systematische Screening neuer potentieller Produzenten. In diesem Rahmen werden am HZI (Helmholtz Zentrum für Infektionsforschung) in Braunschweig seit 1975 verschiedene Stämme von Myxobakterien kultiviert und auf die Produktion biologisch aktiver Verbindungen hin untersucht. Myxobakterien waren bis dahin in dieser Hinsicht praktisch völlig unerforscht, da sie als schwierig zu isolieren und zu kultivieren galten.

\subsection{Morphologie der Myxobakterien}

Myxobakterien besitzen das am weitesten entwickelte Sozialverhalten und die komplexesten Lebenszyklen unter allen bekannten prokaryotischen Organismen. ${ }^{[2]}$ Myxobakterien sind in der Natur sehr verbreitet: Man findet sie in großer Anzahl zum Beispiel in der Erde, auf verrottetem Pflanzenmaterial, im Dung von Pflanzenfressern und auf Baumrinden. Hinsichtlich ihrer Ernährung lassen sich zwei Gruppen unterscheiden. Die meisten Arten weisen einen bakteriolytischen Stoffwechsel auf. Sie sind durch die Exkretion von Exoenzymen in der Lage, Biomakromoleküle abzubauen und können sogar ganze Zellen anderer Mikroorganismen, insbesondere von Bakterien und Hefen, auflösen und die freigesetzten Produkte als Nahrung verwenden. Nur die Vertreter der Gattung Sorangium sind in der Lage, Cellulose abzubauen (cellulolytischer Stoffwechsel).

Die vegetativen Zellen der Myxobakterien sind mit $0.7 \mu \mathrm{m}$ Breite und 3 bis $12 \mu \mathrm{m}$ Länge relativ groß und werden in zwei Typen unterteilt. ${ }^{[3]}$ Die Zellen sind entweder schlank, biegsam und spindelförmig oder stäbchenförmig mit abgerundeten Enden, 
wie zum Beispiel die von Sorangium cellulosum (Abbildung 1). Diese zwei Zelltypen repräsentieren zwei taxonomische Untergruppen der Myxobakterien.

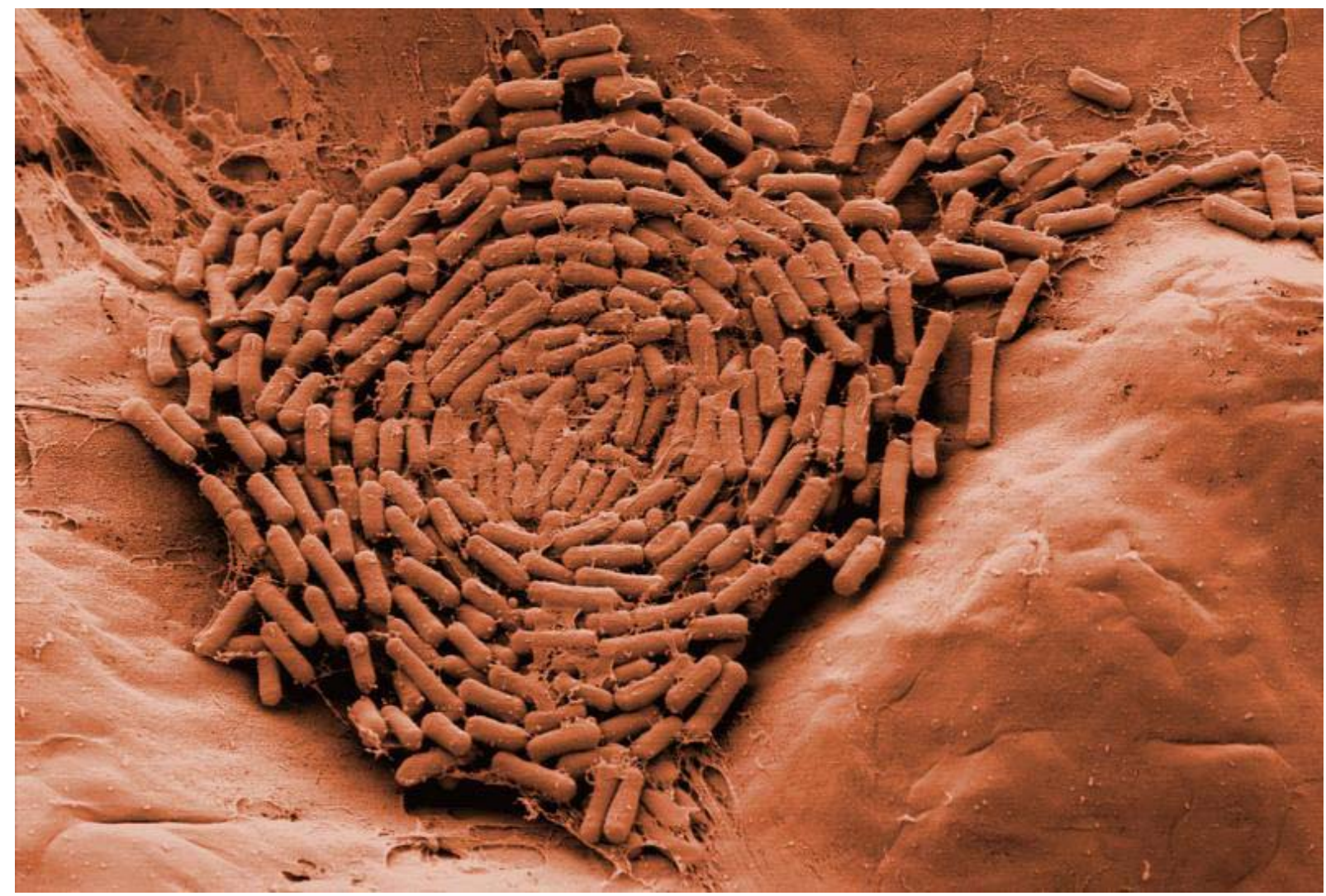

Abbildung 1 Vegetative Zellen von Myxobakterien (Foto: Heinrich Lünsdorf, HZI).

Myxobakterien besitzen die Fähigkeit, sich auf geeigneten Oberflächen durch Gleiten fortzubewegen, sodass man auf festen Medien eine rasche Ausbreitung der Kolonien beobachten kann.

Der erstaunlichste Aspekt der Myxobakterien ist die durch Nahrungsknappheit induzierte Bildung von Fruchtkörpern. Diese Eigenschaft unterscheidet Myxobakterien von allen anderen Prokaryoten und erfordert ein hoch entwickeltes interzelluläres Kommunikationssystem. Unter Mangelbedingungen, zum Beispiel Trockenheit, wandern die vegetativen Zellen an bestimmten Stellen innerhalb der Kolonie aufeinander zu und differenzieren sich zu Fruchtkörpern. Ein einzelner Fruchtkörper kann aus $10^{6}$ oder mehr Zellen bestehen, hat eine Größe von 10 bis $1000 \mu \mathrm{m}$ und ist mit bloßem Auge zu erkennen. Die Fruchtkörper können je nach Gattung ganz unterschiedlich in Form, Größe und Pigmentierung gestaltet sein. Im einfachsten Fall handelt es sich um eine kugelförmige, von Schleim umgebene 
Masse, die sich direkt auf dem Substrat befindet, bis hin zu bizarren Formen, bei denen ein Stiel einen Kopfteil mit fingerartigen Ausstülpungen trägt (Abbildung 2). Bei den meisten Myxobakterien bildet sich um die zusammen gelagerten Zellen herum eine robuste Wand, man spricht dann von Sporangiolen. Die Fruchtkörper der Gattung Sorangium bestehen aus einer mehr oder weniger großen Anzahl von winzigen Sporangiolen, die in dicht gepackten Haufen auf oder in dem Substrat liegen.

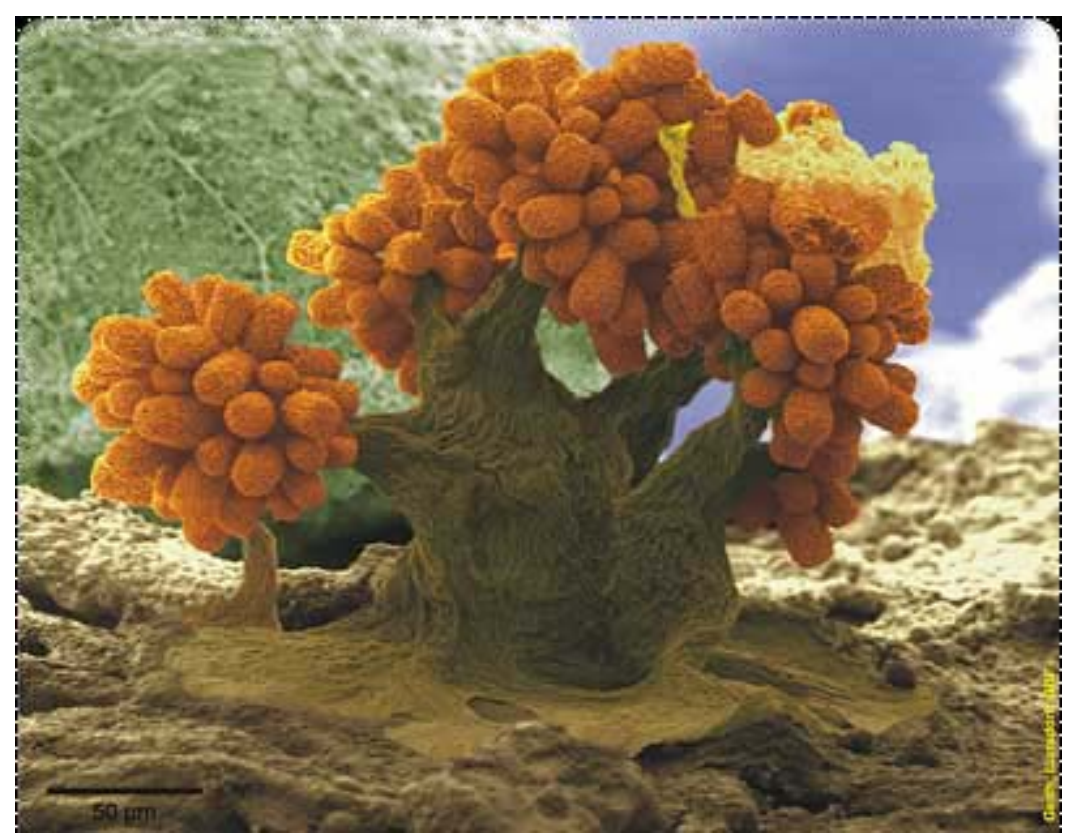

Abbildung 2 Fruchtkörper von Myxobakterien (Foto: Heinrich Lünsdorf, HZI).

Während der Reife unterliegen die vegetativen Zellen im Innern der Fruchtkörper einer Morphogenese zu Ruhezellen, den so genannten Myxosporen. Letztere zeigen eine im Vergleich zu den vegetativen Zellen höhere Resistenz gegen Trockenheit, Hitze und UV-Strahlung. Die Bildung von Myxosporen ermöglicht dem Organismus das Überleben unter ungünstigen Umweltbedingungen. Verbessert sich die Nahrungs- und Umweltsituation, keimen die Myxosporen und gehen wieder in vegetative Zellen über.

Die außergewöhnliche und differenzierte Morphologie erlaubt die Identifizierung von Gattungen und Arten fast gänzlich aufgrund der Charakteristika der vegetativen Zellen, der Myxosporen und Fruchtkörper. ${ }^{[4]}$ 


\subsection{Myxobakterien als Produzenten biologisch aktiver Sekundärmetabolite}

Während sich das Interesse in der Vergangenheit vor allem auf taxonomische und entwicklungsbiologische Aspekte der Myxobakterien konzentriert hatte, wurden sie in den siebziger Jahren als neue Produzentengruppe biologisch aktiver Sekundärmetabolite entdeckt. ${ }^{[1]} 1977$ wurde erstmals die chemische Struktur eines von Myxobakterien produzierten Antibiotikums aufgeklärt: die von dem Stamm Sorangium cellulosum gebildete antifungische Verbindung Ambruticin (Abbildung 3).

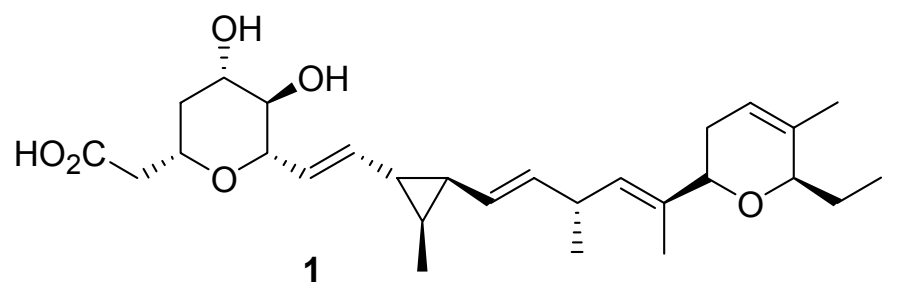

Abbildung 3 Struktur von Ambruticin (1).

Myxobakterien erwiesen sich in den folgenden Jahren als ausgesprochen ergiebige Quelle neuer, biologisch aktiver Verbindungen. In dem seit 1975 am HZI unter der Leitung von H. Reichenbach und G. Höfle durchgeführten Screening-Programm wurden rund 60 neue Grundstrukturen mit 300 Strukturvarianten aufgeklärt, von denen nur drei bereits in der Literatur beschrieben waren und von bereits bekannten Mikroorganismen produziert werden. Die von Höfle et al. gefundenen Substanzen lassen sich ganz unterschiedlichen chemischen Klassen zuordnen. Das Spektrum reicht von macrocyclischen Lactamen und Lactonen über Polyene, Polyether, Aromaten, Chinone und Alkaloide bis hin zu Heterocyclen und Peptiden. Die Fähigkeit, eine bestimmte Verbindung zu produzieren, ist stammspezifisch und keine Eigenschaft der Art. Dies ist ein wichtiger Aspekt angesichts der Tatsache, dass es nur 40 Arten von Myxobakterien gibt, hingegen aber praktisch eine unbegrenzte Anzahl von Stämmen, die ein weites Forschungsfeld bereithalten.

1985 wurde am $\mathrm{HZI}$ aus einer Bodenprobe, die am Ufer des Zambesi-Stromes im Süden Afrikas gesammelt wurde, der Sorangium cellulosum Stamm So ce90 isoliert. ${ }^{[1]}$ Die Kulturbrühe dieses Myxobakteriums zeigte in entsprechenden Screening-Tests eine außergewöhnliche Wirkung und Selektivität gegen Brust- und Dickdarm- Tumorzellinien. Die hierfür verantwortlichen Naturstoffe sind die Epothilone (2a) und (2b) (Abbildung 4). Sie hemmen die Proliferation von 
Tumorzellen. Der Wirkungsmechanismus ist dem von Paclitaxel (Taxol) ähnlich, d. h. es erfolgt ebenfalls eine Hemmung des Abbaus der Mikrotubuli, wodurch nicht funktionsfähige, atypische Mikrotubuli entstehen und sowohl Bildung als auch Funktion der Mitosespindelfaser gestört werden. Die Konsequenz ist, dass Kern- und Zellteilung nicht mehr stattfinden können. Die pharmakologischen Vorteile von Epothilon (2) gegenüber Taxol bestehen in der Wirksamkeit gegen multiresistente Tumorzellen und der wesentlich besseren Wasserlöslichkeit. Dadurch ist eine parentale Applikation ohne Verwendung von Lösungsvermittlern möglich (damit ist eine wesentlich bessere Verträglichkeit verbunden). Taxol wird aus dem Rohextrakt der Rinde der pazifischen Eibe (Taxus brevifolia) gewonnen und ist deshalb nur in begrenztem Maße verfügbar. Die gute synthetische Zugänglichkeit der Epothilone (2) würde die Produktion in großem Maßstab möglich machen und damit eine ausreichende Versorgung von erkrankten Personen sicherstellen.

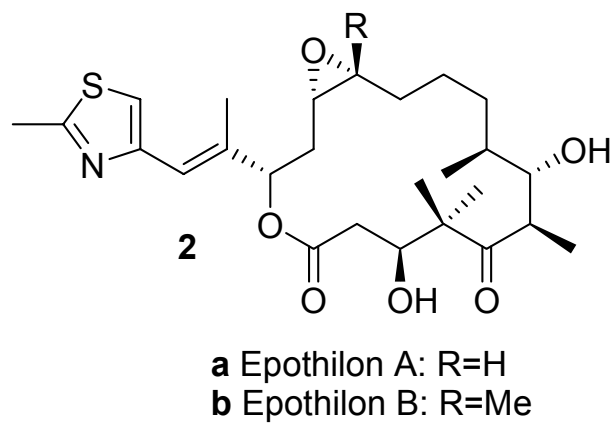

Abbildung 4 Epothilon $A$ (2a) und $B(2 b)$.

Chlorotonil A (3) ist ein tricyclischer Naturstoff, der sich aus einem Decalin-System und einem 14-gliedrigen Macrolacton zusammensetzt (Abbildung 5). Er wurde von Mitarbeitern des HZI, K. Gerth und R. Jansen, im Jahre 2004 aus dem Myxobakterium Sorangium cellulosum isoliert. Sie waren auch maßgeblich an der Aufklärung der Struktur und der absoluten Konfiguration durch Röntgenstrukturanalyse und NMR-Studien beteiligt. ${ }^{[5]}$

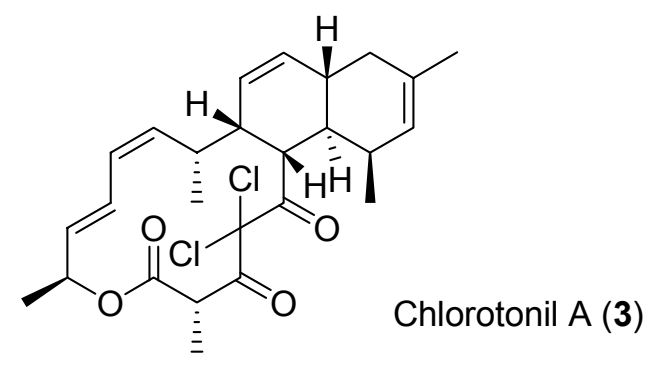

Abbildung 5 Chlorotonil $A$ (3). 
Chlorotonil A (3) weist antibiotische Eigenschaften auf. Wegen der geringen Wasserlöslichkeit ist es im Moment noch nicht möglich, das biologische Potential hinreichend zu erfassen. Dieses Problem soll durch die Darstellung von wasserlöslicheren Derivaten behoben werden (Kapitel 3.10). Aufgrund der Komplexität des Naturstoffs kann davon ausgegangen werden, dass ihm eine wichtige Rolle im Sekundärmetabolismus zukommt und er daher für die Wirkstoffforschung von großem Interesse ist. 


\subsection{Die Bedeutung der Naturstoffsynthese}

Das große wirtschaftliche Interesse an Wirkstoffen verlangt die Produktion der pharmakologisch aktiven Substanzen in großem Maßstab. Oftmals gestaltet sich sowohl die Kultivierung der Naturstoffproduzenten als auch die Extraktion größerer Mengen des Naturstoffes als schwierig. Daher kommt der Totalsynthese von Naturstoffen in der Organischen Chemie eine zentrale Rolle zu. Ziel ist es, durch eine möglichst effiziente Synthese größere Mengen eines viel versprechenden Wirkstoff-Kandidaten zu gewinnen. Dieser kann dann in Rezeptoressays oder durch den Test an speziellen Zelllinien auf einen möglichen therapeutischen Nutzen überprüft werden.

Auch in Fällen, in denen die Gewinnung des Naturstoffes in ausreichenden Mengen über die Kultivierung des Produzenten möglich ist, stellt die Totalsynthese dennoch ein unentbehrliches Werkzeug zur gezielten Modifikation der Leitstruktur und damit zur Darstellung von Analoga dar. Hinzu kommt, dass auf diese Weise eine größere Vielfalt an Derivaten erzeugt werden kann als durch nachträgliche Veränderung des isolierten Naturstoffes. Eine Vielzahl an Analoga ist hilfreich für die Untersuchung von Strukturaktivitätsbeziehungen über biologische Essays. Auf diese Weise können Verbindungen identifiziert werden, die der Leitstruktur in ihrem physikalischen und pharmakologischen Verhalten überlegen sind. ${ }^{[6]}$ 


\section{Aufgabenstellung}

Im Rahmen dieser Dissertation sollte eine möglichst konvergente und effiziente stereoselektive Synthese für den Naturstoff Chlorotonil A (3) ausgearbeitet werden. An die Synthesestrategie wurde außerdem die Anforderung gestellt, dass sie einen einfachen Zugang zu Derivaten ermöglicht, sodass im Anschluss an diese Arbeit insbesondere Analoga mit einer höheren Wasserlöslichkeit dargestellt werden können.

Eine Herausforderung bei der Synthese war der selektive Aufbau der vier tertiären Stereozentren am zentralen Cyclohexenring (Abbildung 6 a). Eine Reaktion, die sich zum Aufbau dieses Strukturmotivs eignet, ist die intramolekulare Diels-Alder Reaktion. Wichtig war in diesem Fall, dass eine Möglichkeit gefunden wird, selektiv das gewünschte von den insgesamt vier möglichen Diastereomeren zu generieren.

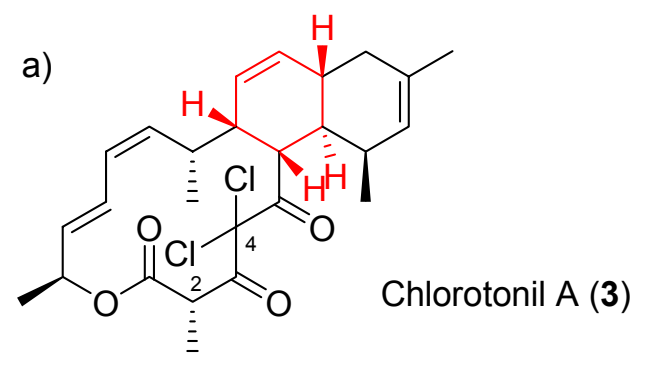

b)<smiles>[R]OC(=O)C(C)C(=O)C(Cl)(Cl)C([R])=O</smiles>

4

\section{Abbildung 6}

a) Chlorotonil $A$ (3), markiert sind die vier tertiären Stereozentren.

b) Chlorierte 1,3-Dicarbonyl-5-ester-Struktureinheit aus Chlorotonil A (3).

Strukturell sehr auffällig bei Chlorotonil A (3) ist die chlorierte 1,3-Dicarbonyl-5-esterStruktureinheit (Abbildung 6 b). Um dieses Strukturelement darstellen zu können, musste nicht nur eine Methode etabliert werden, um das epimerisierungsgefährdete Stereozentrum an $\mathrm{C}(2)$ aufzubauen, sondern auch um selektiv die beiden ChlorSubstituenten an C(4) einzuführen. 


\section{Synthetischer Teil}

\subsection{Retrosynthetische Analyse von Chlorotonil A}

Die effiziente Synthese eines Naturstoffes setzt eine konvergente Strategie voraus. Daher war für Chlorotonil A (3) der folgende retrosynthetische Ansatz nahe liegend: Chlorotonil A (3) kann aus dem Macrolacton 5 durch eine transannulare Diels-Alder Reaktion (TADA) ${ }^{[7]}$ generiert werden (Abbildung 7). Das Macrolacton 5 lässt sich aus vielen kleinen Fragmenten aufbauen. Damit wäre sowohl der Forderung nach einer konvergenten als auch einer leicht zu modifizierende Synthesestrategie Rechnung getragen.

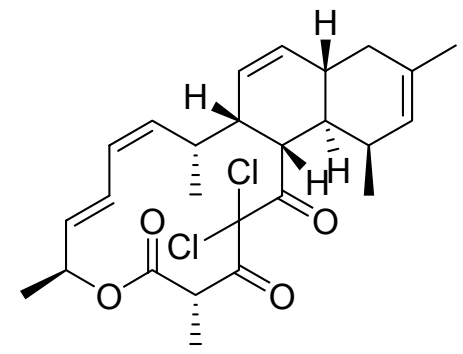

Chlorotonil A (3)
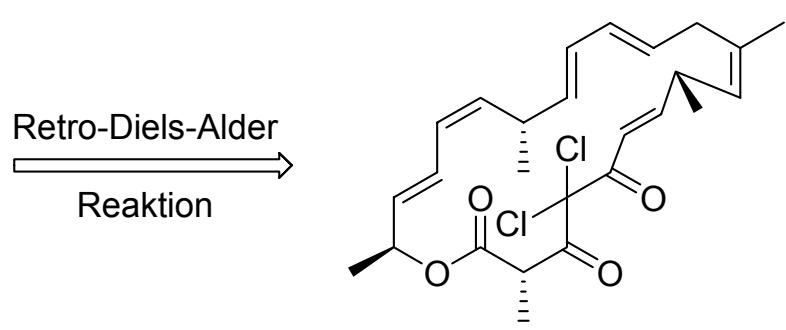

5

Abbildung 7 Diels-Alder Reaktion als Schlüssel- und gleichzeitig finaler Schritt bei der Synthese von Chlorotonil A (3).

Aufgrund der Komplexität des Macrolactons $\mathbf{5}$ ist allerdings kaum vorhersagbar, wie die bevorzugte Konformation im Übergangszustand der Diels-Alder Reaktion aussieht. Man kann nur vermuten, welche Einflüsse sterischer und stereoelektronischer Art den größten Einfluss haben. Entsprechend sind Annahmen zu der Produktverteilung der vier möglichen Diastereomere schwierig. Falls sich herausstellt, dass die Reaktion unselektiv ist oder eines der unerwünschten Diastereomere das Hauptprodukt darstellt, muss diese Strategie verworfen werden. Die Diels-Alder Reaktion ist die letzte Stufe in der Synthese. Daher ist das Risiko groß, dass im Vorfeld viel Zeit in die Synthese des Macrolactons 5 investiert wird und die Darstellung von Chlorotonil A(3) am letzten Schritt scheitert.

Deshalb sollte die Diels-Alder Reaktion zu einem früheren Zeitpunkt der Synthese durchgeführt werden. Möglicherweise sind bei einer weniger komplexen Verbindung die Selektivitäten einfacher zu kontrollieren. Diese Überlegungen führten zur Aufstellung eines modifizierten retrosynthetischen Ansatzes (Abbildung 8): 
Chlorotonil A (3) entsteht aus der Säure 6 durch Macrolactonisierung und anschließender Chlorierung zwischen den Carbonylgruppen. Die Verbindung 6 kann aus der Säure 7 generiert werden.
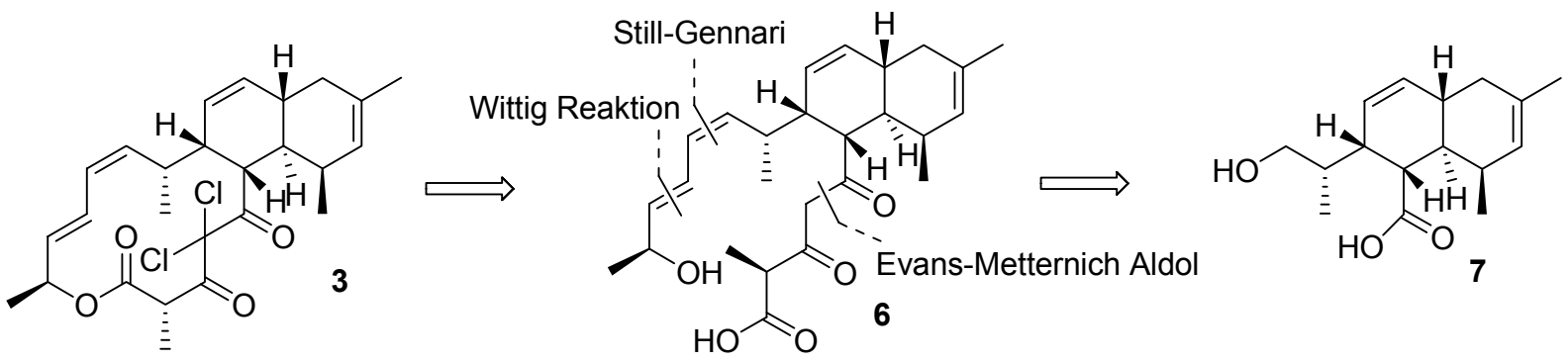
HO 6

Wittig Reaktion
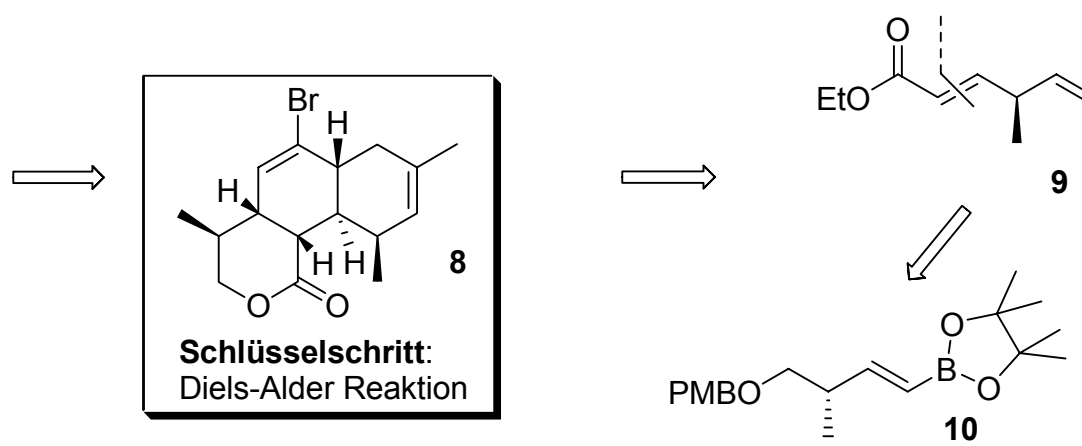

10
Suzuki Kupplung

9

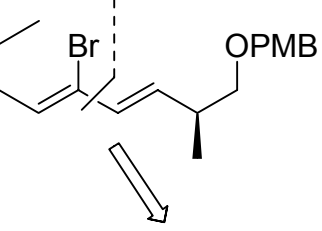<smiles></smiles>

Abbildung 8 Retrosynthese von Chlorotonil A (3).

Dabei wird die Polyen-Seitenkette durch eine Still-Gennari Reaktion ${ }^{[11]}$ und anschließende Wittig Kupplung ${ }^{[12]}$ aufgebaut und die Polyketon-Seitenkette mit Hilfe einer Evans-Metternich-Aldol Reaktion eingeführt. ${ }^{[13]}$ Die Säure 7 entsteht durch die Öffnung des Lactons 8. Das tricyclische System 8 wird durch eine Diels-Alder Reaktion direkt aus dem Ester 9 oder der zuvor cyclisierten Verbindung dargestellt. Der Ester 9 kann durch eine Suzuki Kupplung ${ }^{[14]}$ des Vinylboronsäureesters 10 und des Dibromolefins 11 und anschließende Wittig Reaktion generiert werden. Sowohl der Vinylboronsäureester 10 als auch das Dibromolefin 11 können durch wenige einfache Transformationen aus $\beta$-Hydroxyisobuttersäuremethylester hergestellt werden.

Der Schlüsselschritt der Synthese ist die TADA Reaktion mit dem Makrolacton 12 (Abbildung 9). Möglich ist auch eine intramolekulare Diels-Alder Reaktion (IMDA) mit dem Ester 9. In beiden Fällen sind vier verschiedene Übergangszustände möglich, zwei endo und zwei exo Übergangszustände. Dementsprechend können auch vier verschiedene Diastereomere bei dieser Reaktion gebildet werden (Abbildung 9). 
Es ist anzunehmen, dass die endo Übergangszustände 13 und 14 aufgrund von sekundären Orbitalweches/wirkungen energetisch günstiger sind als die exo Übergangszustände 15 und 16. Daher kann angenommen werden, dass die Bildung der Produkte 17 und 18 gegenüber der von 19 und 20 bevorzugt ist.

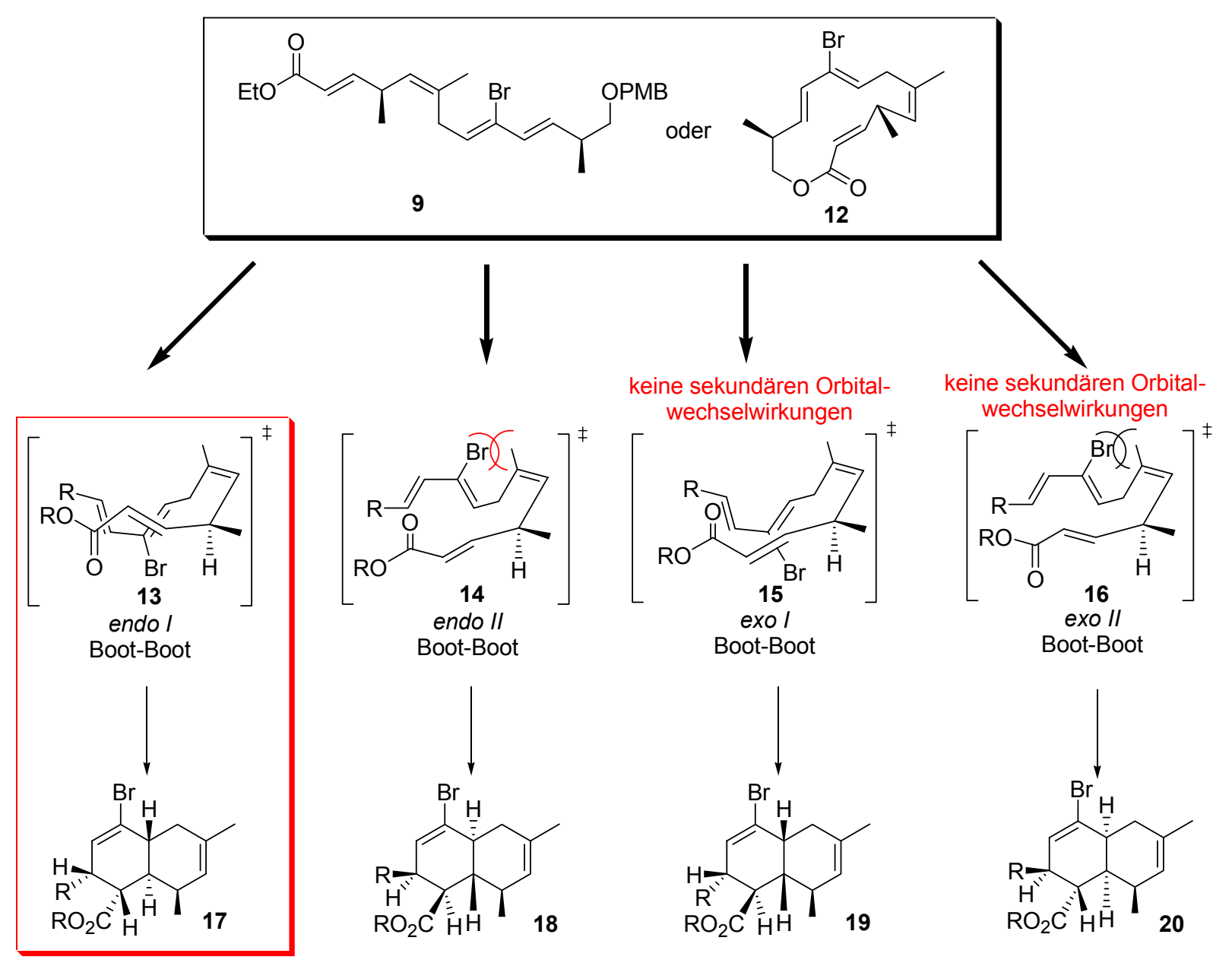

Abbildung 9 Mögliche Übergangszustände der IMDA Reaktion des Esters 9 und des Lactons 12.

Vergleicht man die Struktur der Übergangszustände 13 und 14 miteinander, so stellt man fest, dass bei dem endo-II Übergangszustand 14 sterische Wechelwirkungen zwischen dem Brom-Substituenten und dem Methyl-Substituenten der Z-Doppelbindung auftreten. Daraus kann man schließen, dass der endo-I Übergangszustand energetisch günstiger ist und daher hauptsächlich das gewünschte Produkt 17 gebildet wird. Der Brom-Substituent wurde zu dem Zweck eingeführt, die Produktbildung zu steuern, sodass selektiv die Verbindung 17 entsteht und die Bildung von 18 unterdrückt wird. 


\subsection{Synthese des Vinylboronsäureesters}

Die Synthese des Vinylboronsäureesters 10 ging von D-(-)- $\beta$ Hydroxyisobuttersäuremethylester (21) aus, der zunächst mit einer $p$ Methoxybenzylschutzgruppe (PMB) versehen wurde (Schema 1). Bevorzugt wird diese Schützungsreaktion mit PMB-Trichloracetimidat und Camphersulfonsäure, d.h. säurekatalysiert, durchgeführt. Auf diese Weise werden unerwünschte Retroaldolprozesse von (21) vermieden, die unter Standardbedingungen (z.B. NaH, $\mathrm{PMBCl}$ ) durch Deprotonierung der $\beta$-Hydroxygruppe mit einer starken Base auftreten. Danach wurde der Ester 22 zuerst mit DiBAl-H zum Alkohol 23 reduziert und anschließend durch eine Swern Oxidation zum Aldehyd 24 oxidiert. ${ }^{[15]}$ Beide Stufen sind in hohen Ausbeuten durchführbar (Schema 1).

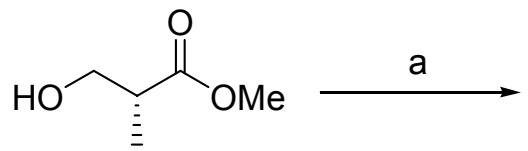

21

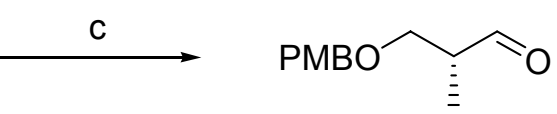

24

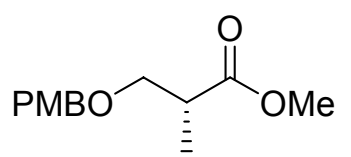

22

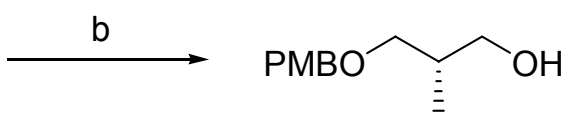

23
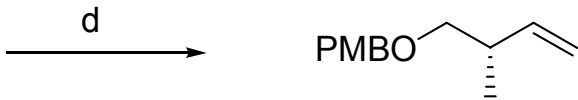

25

Schema 1 Synthese des Alkens 25.

Reaktionsbedingungen: a) (4-Methoxybenzyl)-trichloracetimidat, $\mathrm{CSA}, \mathrm{CH}_{2} \mathrm{Cl}_{2}, \mathrm{RT}, 97 \%$; b) DiBAl-H, $\mathrm{CH}_{2} \mathrm{Cl}_{2},-78{ }^{\circ} \mathrm{C}, 97 \%$; c) Oxalylchlorid, DMSO, $\mathrm{Et}_{3} \mathrm{~N},-78{ }^{\circ} \mathrm{C} \rightarrow \mathrm{RT}, 86 \%$; d) $n$-BuLi, $\left(\mathrm{Ph}_{3} \mathrm{PCH}_{3}\right)^{+} \mathrm{Br}^{-}$, $-78{ }^{\circ} \mathrm{C} \rightarrow \mathrm{RT}, 87 \%$.

Die Oxidation zum Aldehyd 24 ist mit verschiedenen Oxidationsmitteln, wie zum Beispiel Dess-Martin Periodinan oder TPAP/NMO, möglich. Jedoch hat sich die Swern Oxidation gerade für Reaktionen in großem Maßstab als am kostengünstigsten erwiesen. Durch eine Wittig Reaktion mit Methyltriphenylphosphoniumbromid und $n$-Butyllithium wurde dann aus dem Aldehyd 24 das Alken 25 dargestellt.

Damit war das erste Fragment für die Kreuzmetathese generiert, die zum Aufbau des Vinylboronsäureesters 10 (Schema 3) herangezogen werden sollte. Das zweite Fragment, der Boronsäureester 29, wurde synthetisiert, indem die Organomagnesium-Verbindung 26 mit Trimethylborat umgesetzt wurde und die 
entstandene Boronsäure 28 ohne weitere Reinigungsschritte mit Pinakol versetzt wurde. Damit konnte der Boronsäureester 29 in einer Ausbeute von 85\% über drei Schritte erhalten werden. ${ }^{[16]}$

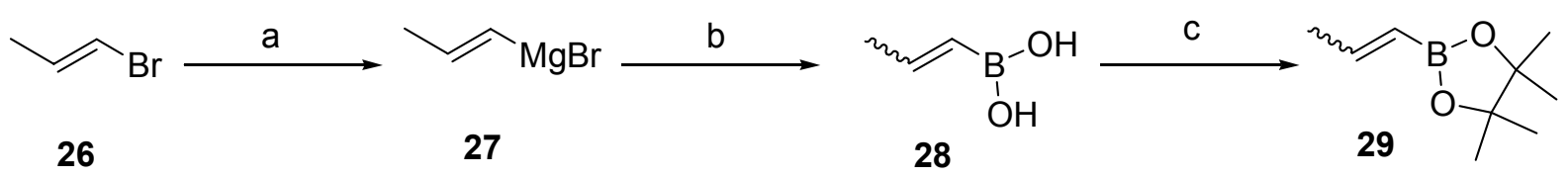

Schema 2 Synthese des Boronsäureesters 29.

Reaktionsbedingungen: a) Mg, THF; b) $\mathrm{B}(\mathrm{OMe})_{3},-78{ }^{\circ} \mathrm{C} \rightarrow 0{ }^{\circ} \mathrm{C}, \mathrm{HCl}$; c) Pinakol, MS $4 \AA$, $85 \%$ über drei Schritte.

Das Alken 25 wurde anschließend mit dem Boronsäureester 29 in einer Kreuzmetathese ${ }^{[16]}$ mit Grubbs Katalysator zweiter Generation zum Vinylboronsäureester 10 umgesetzt, den man in guter Ausbeute und ausgezeichneter E/Z-Selektivität von 50:1 erhielt (Schema 3).

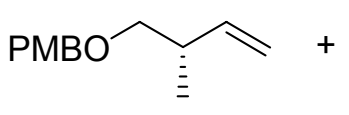

25<smiles>CC=CB1OC(C)(C)C(C)(C)O1</smiles>

29

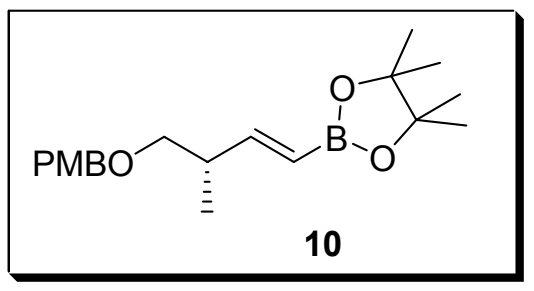

Schema 3 Metathese zur Darstellung des Borosäureesters 10.

Reaktionsbedingungen: a) Grubbs: zweite Generation, $\mathrm{CH}_{2} \mathrm{Cl}_{2}, 16 \mathrm{~h}$ unter Rückfluss, E/Z > 50:1, $81 \%$.

Diese Art der Kreuzmetathese wurde von Grubbs ${ }^{[16]} 2003$ zum ersten Mal beschriebenen. Sie stellt eine allgemeine Methode zur Herstellung von funktionalisierten Vinylboronsäureestern aus verschieden substituierten Alkenen und 1-Propenyl-pinacolboronsäureester 29 dar. Auf diese Weise lassen sich Bausteine für eine Suzuki-Kupplung effizient generieren. 


\subsection{Synthese des Dibromolefins}

Die Synthese des Dibromolefins 11 ging von L-(+)- $\beta$-Hydroxyisobuttersäuremethylester (30) aus, der zunächst mit TBS-Chlorid zum entsprechenden Silylether 31 umgesetzt und anschließend zum Alkohol 32 reduziert wurde (Schema 4).

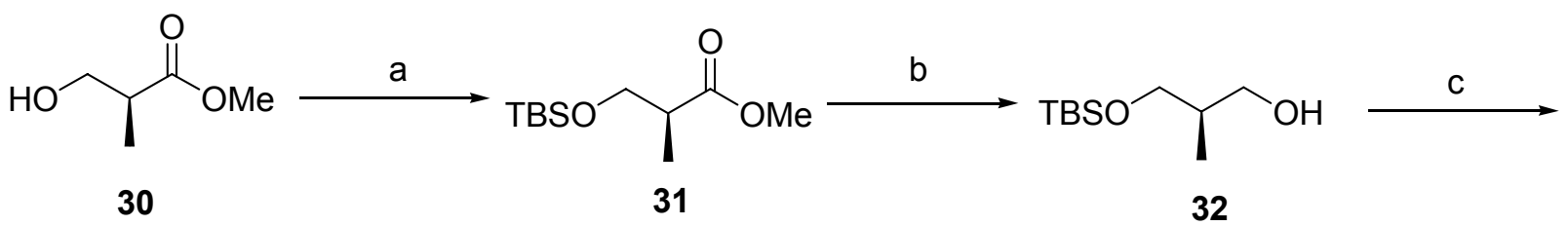<smiles>COC(=O)/C(C)=C\C(C)CO[R15](C)(C)C</smiles>

Schema 4 Synthese des Alkohols 36.

Reaktionsbedingungen: a) TBSCI, DIPEA, DMAP, $\mathrm{CH}_{2} \mathrm{Cl}_{2}, \mathrm{RT}, 96 \%$; b) DiBAI- $\mathrm{H}, \mathrm{CH}_{2} \mathrm{Cl}_{2},-78{ }^{\circ} \mathrm{C}, 91 \%$; c) Oxalylchlorid, DMSO, $\mathrm{Et}_{3} \mathrm{~N},-78{ }^{\circ} \mathrm{C} \rightarrow \mathrm{RT}, 94 \%$; d) $\left(\mathrm{F}_{3} \mathrm{CH}_{2} \mathrm{CO}\right)_{2} \mathrm{P}(\mathrm{O}) \mathrm{CH}\left(\mathrm{CH}_{3}\right) \mathrm{COOCH}_{3} \mathbf{3 5}$, 18-Krone-6, KHMDS, THF, $-40{ }^{\circ} \mathrm{C} \rightarrow-78{ }^{\circ} \mathrm{C}, 85 \%$; e) DiBAI-H, THF, $-50{ }^{\circ} \mathrm{C}, 87 \%$.

Im Anschluss daran wurde der Alkohol 32 durch eine Swern Oxidation zum Aldehyd 33 oxidiert $^{[18]}$ und dann durch die Still-Gennari Variante der Horner-WadsworthEmmons Reaktion ${ }^{[11]}$ in den $\alpha, \beta$-ungesättigten Ester 34 transformiert. Man erhielt in diesem Fall ein Z/E-Verhältnis von 20:1. Der Ester 34 wurde dann mit DiBAl-H reduziert und man konnte den Allylalkohol 36 in guten Ausbeuten isolieren (Schema 4).<smiles>C/C(=C/[C@@H](C)C/C(C)=C\[C@H](C)CO[R15](C)(C)C)CO</smiles>

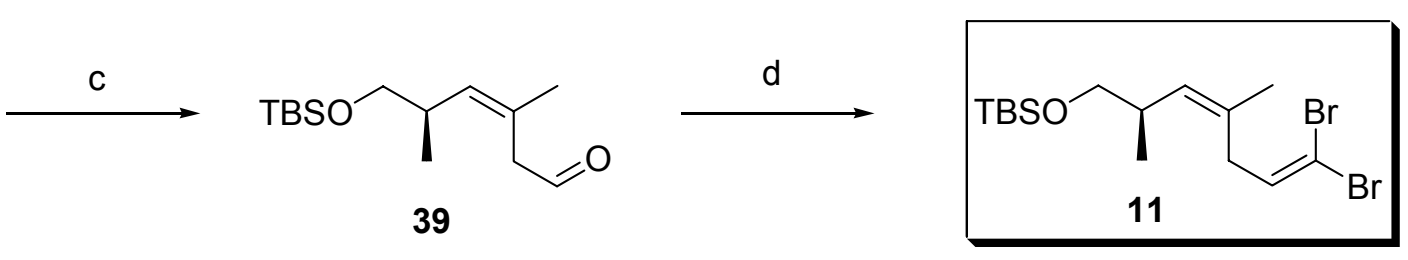

Schema 5 Synthese von Dibromolefin 11.

Reaktionsbedingungen: a) $\mathrm{LiCl}, 2,6$-Lutidin, $\mathrm{CF}_{3} \mathrm{SO}_{2} \mathrm{Cl}$, DMF, 79\%; b) NaCN, DMF, $0{ }^{\circ} \mathrm{C}, 96 \%$ borsm; c) DiBAl-H, $\mathrm{CH}_{2} \mathrm{Cl}_{2}, 81 \%$; d) $\mathrm{CBr}_{4}, \mathrm{PPh}_{3}, \mathrm{Zn}, \mathrm{CH}_{2} \mathrm{Cl}_{2}, \mathrm{RT}, 91 \%$. 
Anschließend wurde der Allylalkohol 36 mit Trifluormethansulfonylchlorid, 2,6-Lutidin und Lithiumchlorid zum Allylchlorid 37 umgesetzt. ${ }^{[19]}$ Mit Natriumcyanid wurde dann das Nitril 38 generiert, das durch die Zugabe von DiBAl-H in den Aldehyd 39 transformiert wurde. Nach einer Corey-Fuchs Reaktion ${ }^{[17]}$ erhielt man schließlich das Dibromolefin 11. 


\subsection{Suzuki Kupplung von Vinylboronsäureester und Dibromolefin}

Die Suzuki Kupplung des Dibromolefins 11 mit dem Vinylboronsäureester 10 wurde nach einer Thalliumethoxid vermittelten Variante von Roush et al. ${ }^{[14,20,21]}$ in einer Ausbeute von $76 \%$ und ausschließlicher Selektivität für die Kupplung mit dem Eständigen Bromid durchgeführt. Die Vorschrift von Roush eignet sich vor allem für 1,1-Dibromolefine und 1-lod-1-trimethylsilylalkene, bei denen als Nebenreaktion häufig die baseninduzierte Eliminierung von Brom- bzw. lodwasserstoff auftritt. Bereits Kishi et al. ${ }^{[22]}$ hatten 1987 festgestellt, dass Thalliumhydroxid verglichen mit anderen häufig eingesetzten Basen, wie zum Beispiel Natriummethanolat oder Kaliumcarbonat, zu einer ca. 1000-fach höheren Reaktionsgeschwindigkeit führt. Roush konnte dieses auch bei Thalliumethoxid feststellen, das im wässrigen Milieu lediglich die Vorstufe von dem in situ gebildeten Thalliumhydroxid ist. Da Thalliumhydroxid sehr licht- und oxidationsempfindlich ist, stellt das wesentlich stabilere Thalliumethoxid eine häufig verwendete Alternative dar. Bei der Suzuki Reaktion mit Thalliumethoxid als Base verlaufen die oben genannten Nebenreaktionen viel langsamer als die Kupplung, sodass in guten Ausbeuten das gewünschte Produkt gebildet wird.

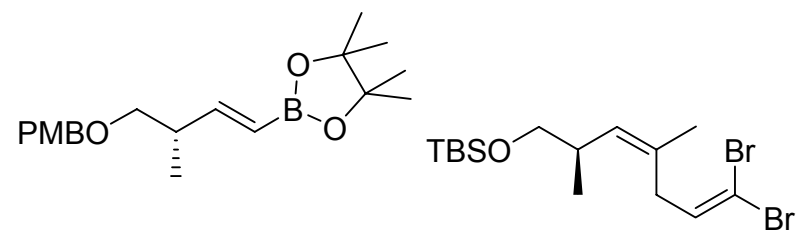

10

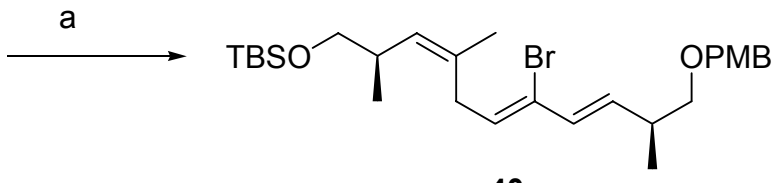

40

Schema 6 Suzuki Kupplung zur Darstellung von Trien 40.

Reaktionsbedingungen: a) $\mathrm{Pd}\left(\mathrm{PPh}_{3}\right)_{4}, \mathrm{TIOEt}, \mathrm{H}_{2} \mathrm{O} / \mathrm{THF}, \mathrm{RT}, 76 \%$.

Die höhere Reaktionsgeschwindigkeit beim Einsatz von Thalliumbasen beruht vermutlich auf der Schwerlöslichkeit der entstehenden Thalliumsalze, wie zum Beispiel Thalliumbromid oder Thalliumiodid. Diese fallen schon bei der Zugabe der Base aus dem THF/Wasser-Gemisch aus. Dadurch wird der geschwindigkeitsbestimmende Schritt der Reaktion, nämlich die Bildung der Organopalladium-Hydroxid Spezies, beschleunigt. Beim Einsatz von Natriummethanolat oder Kaliumcarbonat lösen sich die entstehenden Salze in dem Lösungsmittelgemisch sehr gut, sodass in diesen Fällen keine Gleichgewichtsverschiebung auftritt und die Reaktion wesentlich langsamer abläuft. 
Eine weitere mögliche Nebenreaktion ist bei 1,1-Dibromolefinen die zweifache Kupplung mit der Boronsäure-Spezies. In der Regel verläuft die Reaktion der E-Verknüpfung des Kohlenstoffgerüstes aber wesentlich schneller als die Z-Verknüpfung, sodass bei rechtzeitigem Abbruch der Reaktion nur das gewünschte Produkt $\mathbf{4 0}$ isoliert wird. Es gibt aber auch Fälle, wie zum Beispiel die Synthese von (-)-FR182877 von Evans et al. ${ }^{[23]}$, in denen trotzdem größere Mengen an doppelt gekuppeltem Produkt entstanden sind. Durch das Verwenden einer anderen Base, in diesem Fall Thalliumcarbonat konnte die Nebenreaktion unterdrückt werden. Die Art der Base hat in der Suzuki Reaktion folglich einen großen Effekt auf das Ergebnis der Reaktion und muss auf jede neue Reaktion abgestimmt werden. 


\subsection{Intramolekulare Diels-Alder Reaktion}

Im Anschluss an die Suzuki Kupplung wurde die TBS-Schutzgruppe des Triens 40 mit HF*Pyridin entfernt und der entstandene Alkohol 41 mit Dess-Martin Periodinan zu dem entsprechenden Aldehyd $\mathbf{4 2}$ oxidiert. Eine Wittig Reaktion mit (Triphenylphosphoranyliden)essigsäureethylester führte in guten Ausbeuten und einer E/Z-Selektivität von 50:1 zum a,ß-ungesättigten Ester 9 (Schema 7).<smiles>[R15]OC/C(C)=C\C(Br)=C\C=C\[C@H](C)CO[R5]</smiles>

40<smiles>CC(=O)C[C@H](C)/C=C/C(Br)=C/C/C(C)=C\[C@H](C)C=O</smiles>

42<smiles>[Z14]COC[C@H](C)/C=C/C(Br)=C/C/C(C)=C\[C@H](C)CO</smiles><smiles>CCOC(=O)/C=C/[C@@H](C)/C=C(/C)C/C=C(Br)/C=C/[C@H](C)CO[Mg]</smiles>

9

Schema 7 Synthese des Esters 9.

Reaktionsbedingungen: a) $\mathrm{HF}^{*}$ Pyridin, THF/Pyridin = 1:1, 96\%; b) Dess-Martin Periodinan, $\mathrm{CH}_{2} \mathrm{Cl}_{2}$, $87 \%$; c) $\mathrm{PPh}_{3}=\mathrm{CHCOOEt}, \mathrm{CH}_{2} \mathrm{Cl}_{2}, 89 \%$.

Zur Generierung des Lactons 12 wurde die PMB-Schutzgruppe von 9 mit DDQ entfernt und der entstandene Hydroxyester 43 mit Lithiumhydroxid verseift. Eine anschließend durchgeführte Yamaguchi Macrolactonisierung ${ }^{[8]}$ ergab das gewünschte Zielmolekül 12 (Schema 8).<smiles>CCOC(=O)C=C/C(C)=C\C/C(C)=C/C(C)/C=C/C(C)/C=C/C(=O)OCC</smiles>

9<smiles>CC(C=C[C@@H](C)CO)=CCC(C)=CC(C)C=CC(=O)O</smiles>

44
43<smiles>CC1=CC=CC(C)COC(=O)C(C)=CC(C)=CC=C1</smiles>

12

\section{Schema 8 Synthese des Lactons 12.}

Reaktionsbedingungen: a) DDQ, $\mathrm{CH}_{2} \mathrm{Cl}_{2} / \mathrm{H}_{2} \mathrm{O}=18: 1, \mathrm{RT}, 97 \%$; b) $\mathrm{LiOH}, \mathrm{THF} / \mathrm{H}_{2} \mathrm{O} / \mathrm{MeOH}=3: 1: 1$;

c) TCBC, DMAP, $\mathrm{Et}_{3} \mathrm{~N}, \mathrm{RT}, 56 \%$. 
Der Ester 9 und das Lacton 12 wurden nun beide in der geplanten Diels-Alder Reaktion umgesetzt. $^{[7]}$ Den Literaturbeispielen ${ }^{[7,23,24,25]}$ entsprechend wurde für die TADA Reaktion des Lactons 12 das tricyclische Lacton 8 erwartet. Für die IMDA Reaktion des offenkettigen Ethylesters 9 das bicyclische Produkt 45 (Schema 9).<smiles>CCOCC(C)C=CC(Br)=CCC(C)=CC(C)C=CC(=O)OCC</smiles><smiles>CC1=CC(C)C2C(Br)=CC3[C@H](C)COC(=O)[C@@H]3[C@H]2C1</smiles>

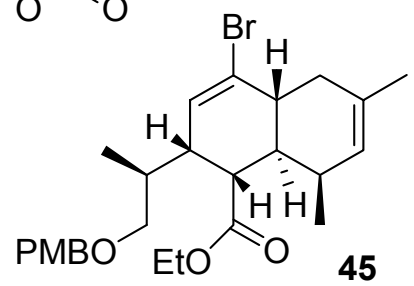

Schema 9 Erwartete Ergebnisse für die Diels-Alder Reaktion von Lacton 12 und Ester 9.

Für diese Umsetzungen wurde der Ester 9 bzw. das Lacton 12 in Toluol gelöst, mit $\mathrm{BF}_{3}{ }^{*} \mathrm{OEt}_{2}$ versetzt und anschließend für drei Stunden auf $85{ }^{\circ} \mathrm{C}$ in einem geschlossenen Gefäß ohne Druckausgleich erhitzt. Es wurde festgestellt, dass man unabhängig vom eingesetzten Vorläufer Lacton 8 als Hauptprodukt erhält (Schema 10).<smiles>CC(C)=CCC=C(C)CC=CC(=O)OCC(C)C</smiles>

12

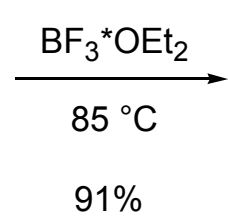

$91 \%$

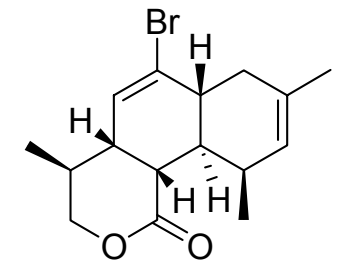

8<smiles>CC1=CC(C)[C@]2(C)C(C=CC3[C@@H]2C(=O)OC[C@@H]3C)C1</smiles>

46

Diastereomerenverhältnis 12:1

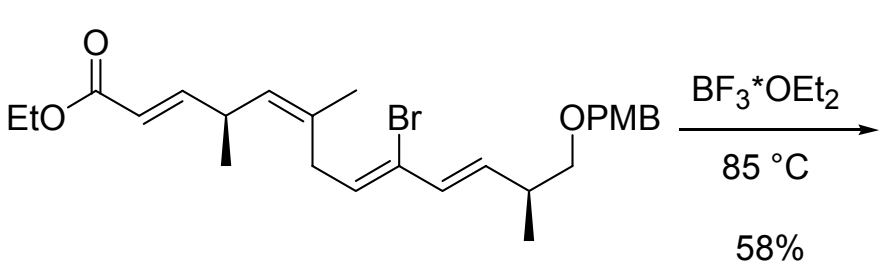

9

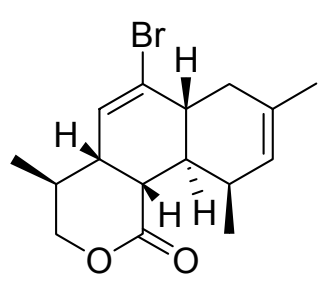

8<smiles>CC1=CC(C)[C@]2(C)C(C1)C(Br)=CC1C2C(=O)OC[C@@H]1C</smiles>

46

Diastereomerenverhältnis 13:1

Schema 10 Tatsächliche Ergebnisse der Diels-Alder Reaktion des Lactons 12 und des Esters 9. 
Dieses Ergebnis zeigt, dass die PMB-Schutzgruppe des Esters 9 durch die LewisSäure entfernt wurde. Durch nachfolgende Umesterung und Diels-Alder Reaktion entsteht schließlich das Lacton 8. Die Reihenfolge der beiden letzten Schritte ist nicht geklärt. Daher kann es sich bei der Diels-Alder Reaktion sowohl um die transannulare Variante handeln als auch um die Diels-Alder Reaktion, die von dem offenkettigen System ausgeht.

In beiden Fällen (Schema 10) wurde das gewünschte Lacton 8 (endo-I Produkt) mit guten Diastereoselektivitäten von $\mathbf{8 : 4 6}=12: 1$ bzw. 13:1 erhalten. Durch Röntgenstrukturananlyse konnte die absolute Konfiguration des Hauptproduktes 8 und auch des diastereomeren Nebenproduktes 46 (endo-Il Produkt) bestätigt werden (Abbildung 10).
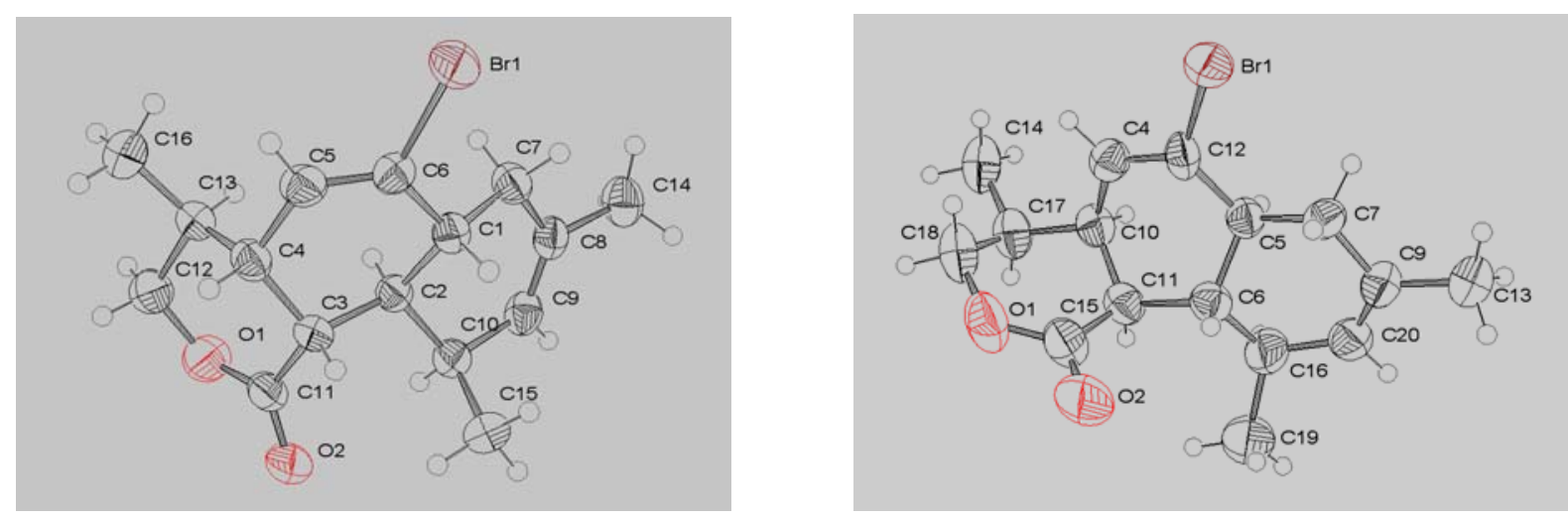

Abbildung 10 ORTEP Darstellung der Röntgenstrukuren der Diels-Alder Produkte.

Links: das Hauptprodukt 8

Rechts: das Nebenprodukt 46

Die Synthese des tricyclischen Lactons 8 über die Kaskadenreaktion aus dem offenkettigen Ester 9 ist trotz der mäßigen Ausbeute von 58\% sehr elegant. Verglichen mit dem Aufbau des Lactons 12 vor der Diels-Alder Reaktion können drei Stufen eingespart werden.

Bei der Diels-Alder Reaktion von $\mathbf{9}$ ist die Temperaturabhängigkeit des Diastereomerenverhältnisses bemerkenswert. Es konnte festgestellt werden, dass der Anteil des endo-II Produktes 46 bei tieferen Temperaturen zunimmt und bei Temperaturen unterhalb von $25{ }^{\circ} \mathrm{C} 46$ Hauptprodukt wird (Tabelle 1). Daraus kann geschlossen werden, dass das endo-I Produkt 8 das thermodynamische Produkt darstellt. Das Diastereomernverhältnis der Reaktionsprodukte kann sehr leicht anhand des ${ }^{1}$ HNMR bestimmt werden (Abbildung 11). 
Tabelle 1 Temperaturabhängigkeit des Diastereomerenverhältnisses bei der DielsAlder Reaktion von Ester 9.

\begin{tabular}{|c|c|c|c|}
\hline Temperatur $\left[{ }^{\circ} \mathrm{C}\right]$ & Diastereomerenverhältnis 8:46 & Zeit [h] & Ausbeute [\%] \\
\hline 85 & $13: 1$ & 3 & 58 \\
\hline 65 & $5: 1$ & 5 & 47 \\
\hline 25 & $1: 1$ & 10 & 34 \\
\hline-40 & $1: 4$ & 24 & 13 \\
\hline
\end{tabular}
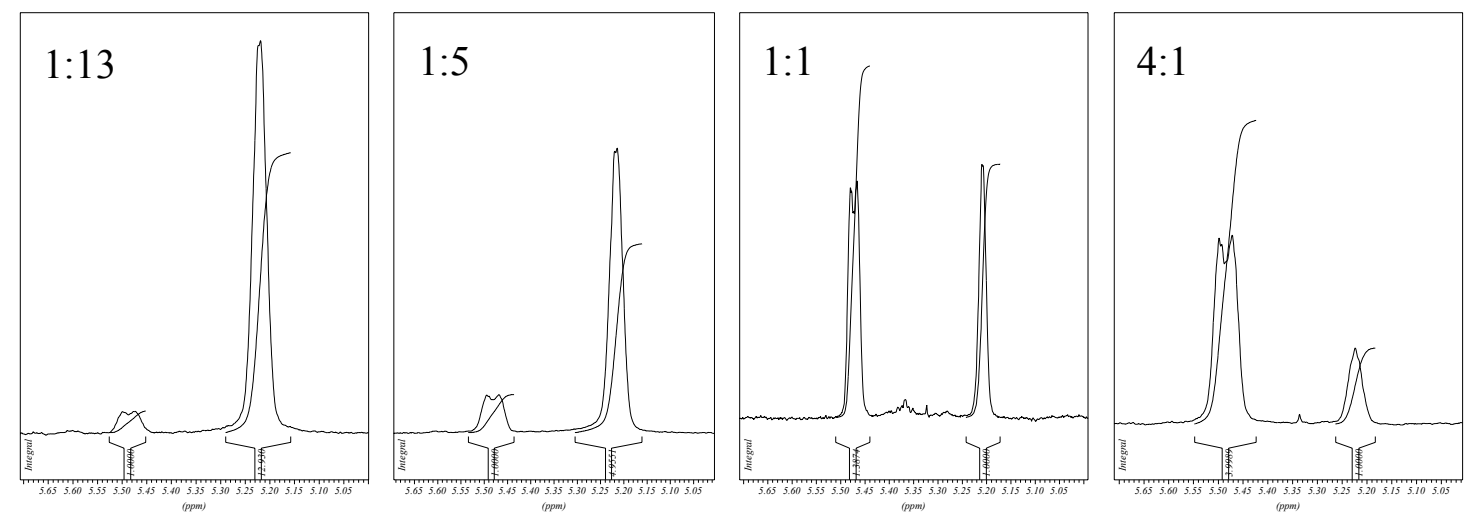

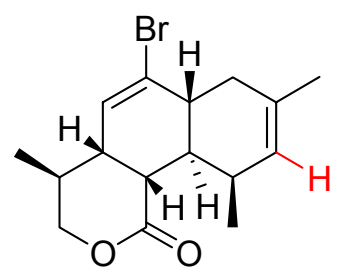

16-endo-I

${ }^{1}$ HNMR: 5.2 ppm<smiles>CC1=CC[C@H](C)[C@]2(C)C(=O)OC[C@H](C)[C@H]2C=C1Br</smiles>

17-endo-II

${ }^{1}$ HNMR: 5.48 ppm

Abbildung 11 Bestimmung des Diastereomerenverhältnisses von 8 und 46 über ${ }^{1}$ HNMR-Analyse durch die Integration des in der Zeichnung rot markierten Protons.

Die Strategie für die Diels-Alder Reaktion zur Steigerung der Selektivitäten war, als stereodirigierende Gruppe einen Brom-Substituenten einzuführen. Damit sollte bevorzugt das endo-I Produkt 8 gebildet werden, da die Übergangszustände für die anderen möglichen Produkte energetisch angehoben werden. Dieses Konzept wurde von Wilson et al. ${ }^{[26]}$ als erstes angewandt. Er konnte zeigen, dass an $\mathrm{C}(8)$ methylsubstituierte Systeme, wie zum Beispiel das Trien 47, bevorzugt das trans verknüpfte Produkt 48 bilden. Demgegenüber verläuft die Reaktion bei Trienen ohne Methyl-Substituenten unselektiv (Schema 11). Diese Beobachtung erklärte er 
anhand der vermuteten Übergangszustände. Die Diels-Alder Reaktion des an C(8) methylsubstituierten Triens 47 verläuft bevorzugt über den Übergangszustand 50transoid. In dem alternativen Übergangszustand 49-cissoid führen abstoßende sterische Wechselwirkungen zwischen der Methylgruppe und einem pseudo axialen Wasserstoffatom dazu, dass er energetisch höher liegt. Daher ist Verbindung 48, die aus dem Überganszustand 50-transoid resultiert, das Hauptprodukt der Reaktion (Schema 11).

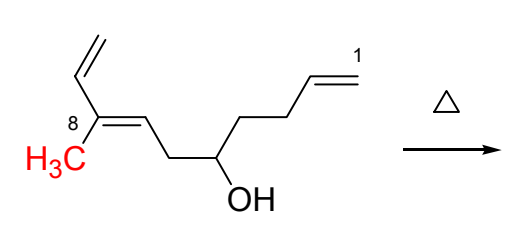

47

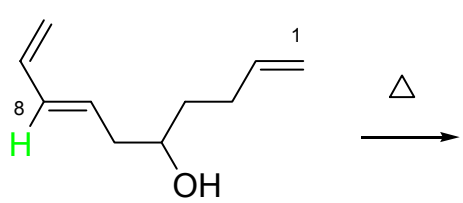

51

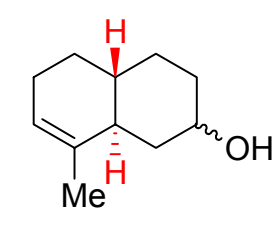

48

$>95 \%$ trans verknüpft<smiles>O[C@H]1CC[C@@H]2CCC=CC2C1</smiles>

52
Übergangszustände

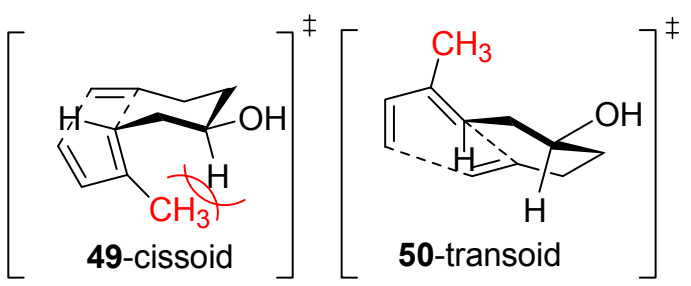

55:45 trans:cis

Schema 11 Konzept der stereodirigierenden Gruppe von Wilson.

Das Konzept von Wilson wurde unter anderem von Roush et al. ${ }^{[27]}$ weiter ausgearbeitet und bei einigen Naturstoffsynthesen, u.a. bei der Synthese von Chlorothricolid, erfolgreich eingesetzt. Roush untersuchte ausführlich das TrienSystem 53 (Abbildung 12). Wird dieses in Toluol erhitzt, so erhält man ein Gemisch aller vier möglichen Produkte, wobei für keine Verbindung eine gute Selektivität gegeben ist. Nach der Einführung eines Brom-Substituenten am Diensystem erreicht man eine gute Selektivität für das endo-I Produkt 55 (Schema 12).

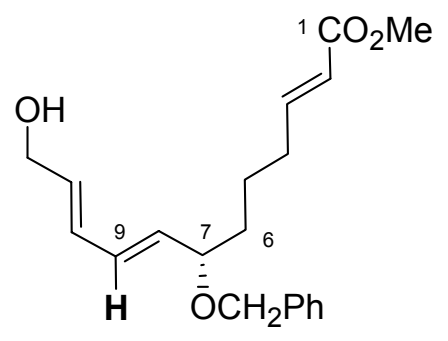

53

Abbildung 12 Von Roush et al. untersuchtes Trien 53. 


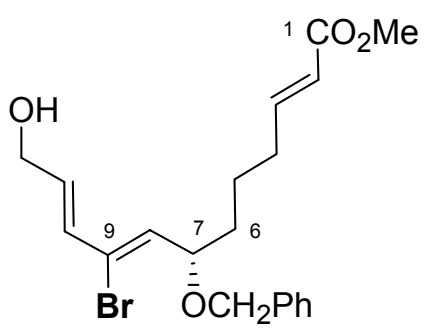

54
Toluol, $180^{\circ} \mathrm{C}$

$79 \%$

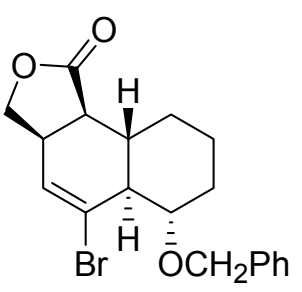

55

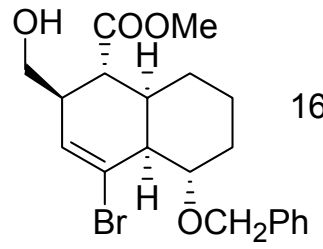

57
$70: 11$<smiles>O=C1OC[C@H]2C=C(Br)[C@@H]3[C@@H](CCC[C@H]3O)[C@H]12</smiles>

56

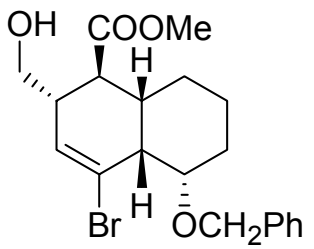

58

Schema 12 Beispiel für das Konzept der stereodirigierenden Gruppe von Roush. ${ }^{\text {[7] }}$

Roush formulierte als Argumentationshilfe die Übergangszustände der vier möglichen Produkte (Abbildung 13). Im endo-II Übergangszustand 60 treten abstoßende Wechselwirkungen zwischen dem Brom-Substituenten an C(9) und der $\mathrm{OCH}_{2} \mathrm{Ph}-G r u p p e$ an $\mathrm{C}(7)$ auf. Die exo Übergangszustände 61 und 62 sind durch Wechselwirkungen zwischen Brom-Substituent und einem axial stehenden Wasserstoffatom destabilisiert. Deshalb ist der endo-I Übergangszustand 59 favorisiert und das Lacton $\mathbf{5 5}$ das Hauptprodukt (Abbildung 13).

endo Übergangszustände

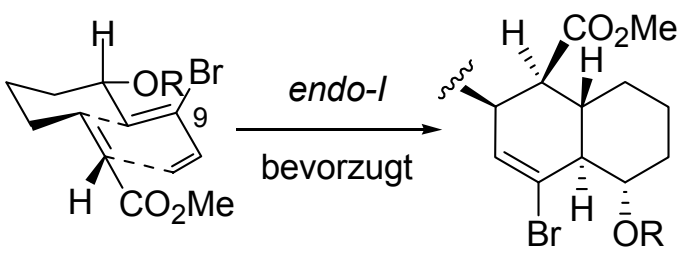

59

55 exo Übergangszustände

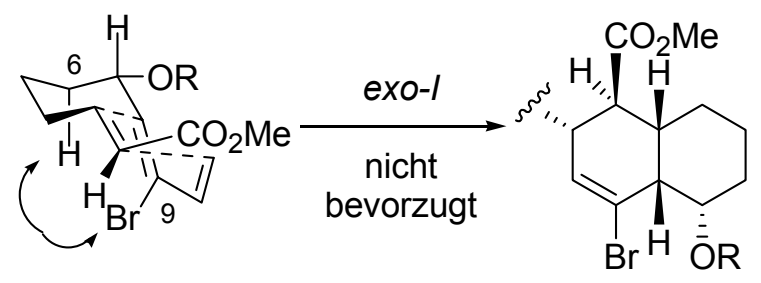

58

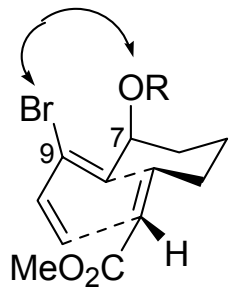

60

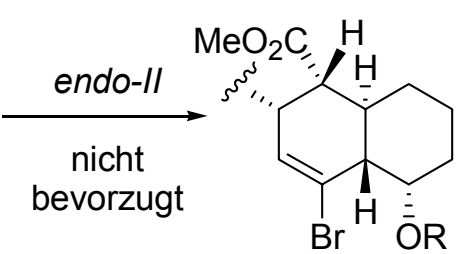

56

$$
\mathrm{R}=\mathrm{CH}_{2} \mathrm{Ph}
$$

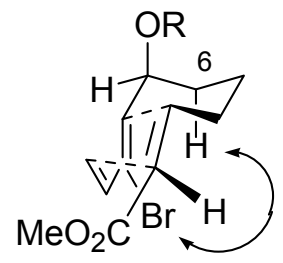

62

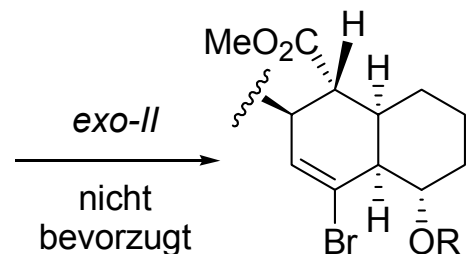

57

Abbildung 13 Von Roush formulierte Übergangszustände. ${ }^{[27]}$ 
Bei den retrosynthetischen Erläuterungen zu Chlorotonil A (Kapitel 3.1) wurden im Sinne des Konzeptes der stereodirigierenden Gruppe Voraussagen zu dem Verlauf der Diels-Alder Reaktion gemacht. Die experimentellen Ergebnisse der Diels-Alder Reaktion mit Ester 9 und Lacton 12 sprechen auf den ersten Blick dafür, dass das Konzept der stereodirigierenden Gruppe eine hinreichende Erklärungen liefert. Als Hauptprodukt wird das gewünschte endo-I Produkt 8 erhalten. Allerdings steht die Tatsache, dass sich das Produktverhältnis endo-I $8 \mathrm{zu}$ endo-Il 46 bei tieferen Temperaturen umkehrt, im Widerspruch zu den Vermutungen, dass der endo-I Übergangszustand 13 den energetisch günstigsten darstellt. Trotz der angenommenen Wechselwirkungen zwischen Brom-Substituent und Methylgruppe am Z-Alken (s. Kapitel 3.1, Abbildung 9) scheint der endo-Il Übergangszustand 14 energetisch bevorzugt zu sein. Offenbar handelt es sich bei dem endo-I Produkt 8 um das thermodynamische Produkt, da es bei hohen Temperaturen erhalten wird.

Ein weiteres Beispiel für thermodynamische Reaktionskontrolle bei einer Diels-Alder Reaktion ist die Synthese von (-)-Oblongolid (64) von Shing und Yang. ${ }^{[2]}$ Sie generierten ein ähnliches tricyclisches System wie Lacton 8. Allerdings wird bei Shing und Yang das endo-II Produkt (64) unter thermodynamischer Reaktionskontrolle gebildet.

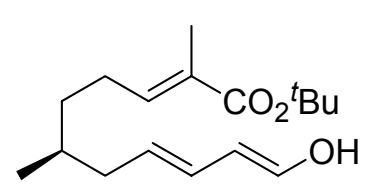

63

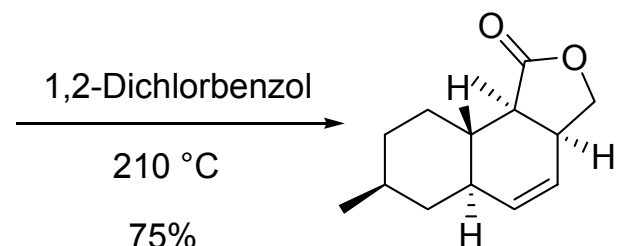

64

Schema 13 Diels-Alder Reaktion bei der Synthese von (-)-Oblongolid (64).

Im Unterschied zu der Synthese von Chlorotonil A (3) wird keine Lewis-Säure verwendet, woraus die unterschiedlichen Ergebnisse resultieren können. Von Marshall et al. ${ }^{[29]}$ konnte gezeigt werden, dass das Produktverhältnis bei einer DielsAlder Reaktion stark variiert, wenn unterschiedliche Lewis-Säuren eingesetzt werden. Ähnliche Beobachtungen bezüglich der Selektivität werden auch gemacht, wenn Lewis-Säure katalysierte Diels-Alder Reaktionen mit unkatalysierten verglichen werden. Bei der Umsetzung von Ester 9 bzw. Lacton 12 erreicht man ohne das 
Hinzufügen einer Lewis-Säure kaum Umsatz. Daher sind Untersuchungen wie die von Marshall et al. angestellten in diesem Fall nicht möglich.

Eine andere Erklärung für die unterschiedliche Produktverteilung nach den DielsAlder Reaktion von Ester 9 und Ester 63 könnte die unterschiedliche Funktionalisierung der beiden Verbindungen sein. Ester 9 weist neben dem Diensystem und der Doppelbindung, die als Dienophil reagiert, eine weitere Doppelbindung auf. Diese verhindert wahrscheinlich einen optimalen sesselförmigen Übergangszustand, während er bei dem Ester 63 vermutlich möglich ist. Auch Unterschiede im Substitutionsmuster können sich mitunter auf die Diastereomerenverteilung auswirken.

Um die Notwendigkeit des Brom-Substituenten am Diensystem bei der Diels-Alder Reaktion von Ester $9 \mathrm{zu}$ überprüfen, wurde der Brom-Substituent mit NatriumQuecksilberamalgam entfernt ${ }^{[27]}$ und der daraus resultierende Ester 65 unter den Bedingungen der intramolekularen Diels-Alder Reaktion eingesetzt. Das Ergebnis ist ein 3:1:1 Verhältnis von endo-I Produkt 66 : endo-II Produkt 67 : exo-II Produkt 68 (Schema 14). Nicht nur die Ausbeute ging bei der Verwendung des Esters 65 statt des Esters 9 zurück, sondern auch die Diastereoselektivität. Verglichen mit dem Produktverhältnis, das aus der Diels-Alder Reaktion mit dem Ester 9 resultierte, nahm die Bildung des unerwünschten endo-Il Produktes $67 \mathrm{zu}$ und zusätzlich wurde das unerwünschte exo-II Produkt 68 gebildet.<smiles>CCOC[C@@H](C)/C=C/C=C/C/C(C)=C\[C@@H](C)/C=C/C(=O)OCC</smiles>
65

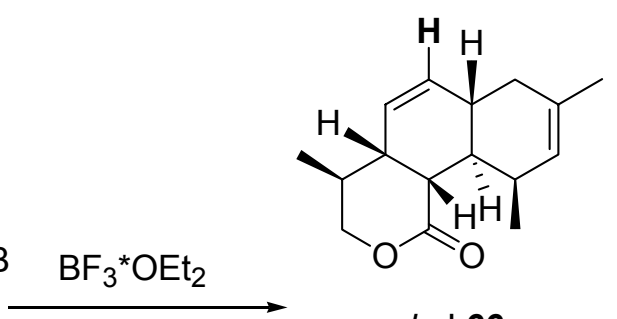

endo-l 66<smiles>CC1=C[C@H](C)[C@H]2[C@H](C=C[C@@H]3CCOC(=O)[C@@]32C)C1</smiles>

exo-II 68 $49 \%$

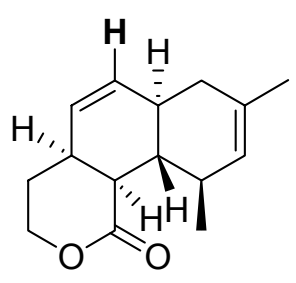

endo-II 67

Diastereomerenverhältnis:

$66: 67: 68=3: 1: 1$

Schema 14 Diels-Alder Reaktion ohne Brom-Substituent am Diensystem. 
Anscheinend konnte die Bildung des exo-II Produktes durch den Brom-Substituenten am Diensystem des Esters 9 verhindert werden. Diese Beobachtung entspricht denen von Roush et al. gemachten (Schema 12). Allerdings sind die von ihm vorgestellten Übergangszustände (Abbildung 13) nicht ohne weiteres auf das System 9 übertragbar. Wegen der zusätzlichen Doppelbindung im Ester 9 können keine optimalen sesselförmigen Übergangszustände ausgebildet werden. Wahrscheinlicher ist, dass bootförmige Übergangszustände durchlaufen werden (Abbildung 9). Aus den experimentellen Ergebnissen der Reaktionen mit den Estern 9 und 65 konnte geschlossen werden, dass bei der Diels-Alder Reaktion mit dem Brom-Substituenten am Dien-System in den exo Übergangszuständen entweder sterische Wechselwirkungen oder elektronische Effekte auftreten, die für die höhere Energie dieser Übergangszustände verantwortlich sind. Diese sterischen oder elektronischen Effekte scheinen bei der Diels-Alder Reaktion ohne Brom-Substituent am DienSystem nicht vorhanden zu sein, sodass die Reaktion unselektiver wird.

Bei dem Lacton 8 handelt es sich vermutlich um das thermodynamische Produkt. Wenn die Diels-Alder Reaktion reversibel sein sollte, könnte man durch Verlängerung der Reaktionszeit oder Erhöhung der Reaktionstemperatur aus dem Ester $\mathbf{9}$ das Lacton $\mathbf{8}$ diastereomerenrein gewinnen. In der Synthese von (-)-Oblongolid (64) von Shing und Yang konnte eine solche Reversibilität gezeigt werden. Daher wurde das Lacton 46 (endo-II Produkt) in die IMDA eingesetzt (Schema 15). Man erhielt unter den identischen Reaktionsbedingungen wie bei der Umsetzung von Ester 9 ein 8:1 Gemisch des endo-I Produktes 8 zum endo-II Produkt 46. Damit konnte für diese IMDA Reaktion die Reversibilität bewiesen werden. Allerdings kann die Reaktionsdauer nicht beliebig verlängert oder die Reaktionstemperatur nicht beliebig erhöht werden, da sonst Zersetzungsprozessen auftreten.

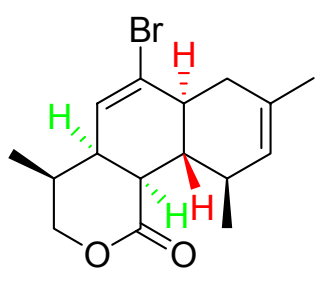

endo-II 46

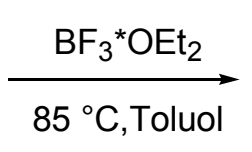

$85^{\circ} \mathrm{C}$ Toluo

Schema 15 Reversibilität der Diels-Alder Reaktion. 
Zusammenfassend kann man bei den Betrachtungen zu der Diels-Alder Reaktion sagen, dass die Voraussage der Produktverteilung schwierig bleibt, vor allem wenn das vorliegende System noch nicht genauer untersucht wurde. Das Ergebnis kann durch sehr viele Faktoren, wie z.B. sterische oder elektronische Effekte und die Art der Durchführung (mit/ohne Lewis-Säure, Temperatur, Lösungsmitteleffekte), beeinflusst werden. 


\subsection{Aufbau der Dien-Seitenkette}

Nach der erfolgreich durchgeführten IMDA Reaktion sollte ausgehend vom Lacton 8 die Dien-Seitenkette aufgebaut werden. Deshalb wurde am Diels-Alder Produkt 8 der Brom-Substituent entfernt. Dieses war mit Natrium-Quecksilber Amalgam in guten Ausbeuten möglich (Schema 16). ${ }^{[27]}$ Im Anschluss daran sollte das Lacton 66 geöffnet werden. Dabei stellte sich heraus, dass Lacton 66 mit $\mathrm{LiOH}$ oder $\mathrm{KOH}$ verseift werden kann, aber schon während der Aufarbeitung das Lacton 66 zurückgebildet wird. Die Ursache hierfür ist die räumliche Nähe der Säure- und Alkoholfunktion. Selbst wenn in situ die Säure zum Methylester umgesetzt wurde, bildete sich nach dem Isolieren nach kurzer Zeit das Lacton 66 zurück. Daher wurde schließlich eine Methode gewählt, bei der das Lacton 66 geöffnet und die Alkoholfunktion in situ geschützt wurde. ${ }^{[30]}$ Die Säure 69 konnte dann mit DiBAl-H reduziert werden. Der entstandene Alkohol $\mathbf{7 0}$ wurde mit TBDPSCI in den Silylether 71 überführt. Im Anschluss daran musste die Benzylschutzgruppe wieder entfernt werden, was mit $\mathrm{BCl}_{3}{ }^{*} \mathrm{SMe}_{2}$ gelang. ${ }^{[31]}$

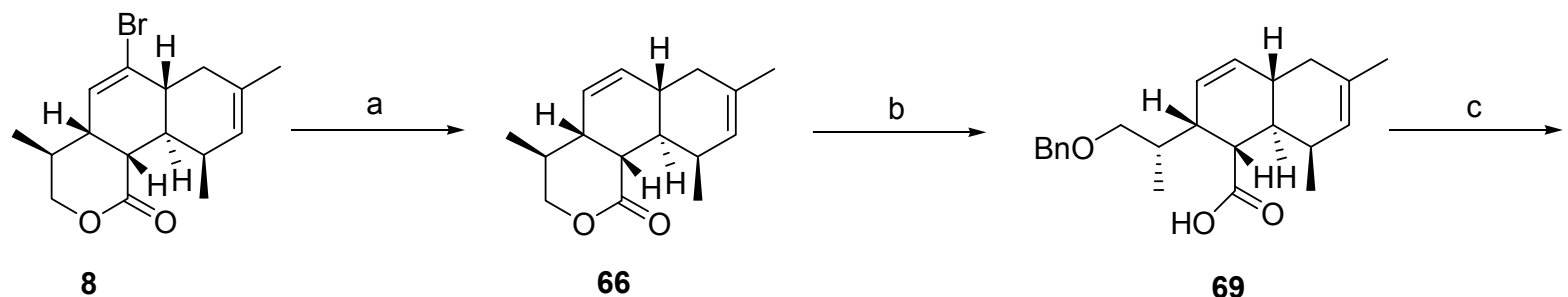

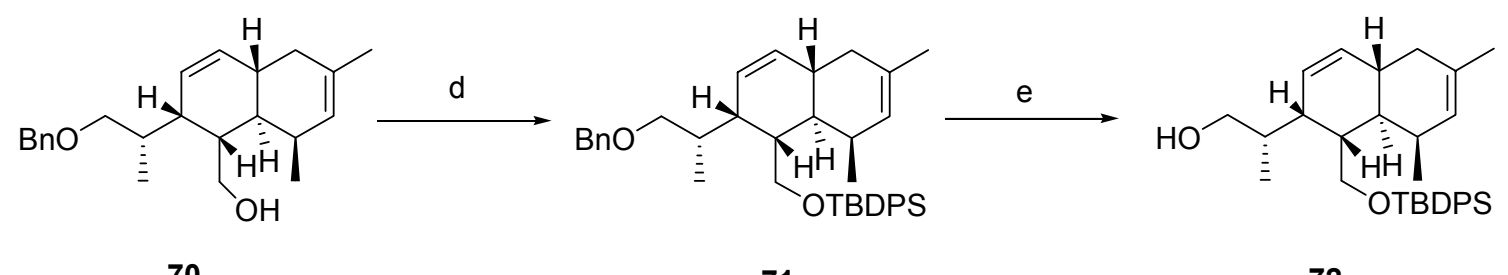

70

71

72

\section{Schema 16 Synthese des Alkohols 72.}

Reaktionsbedingungen: a) Na/Hg, $\mathrm{MeOH}, 92 \%$; b) $\mathrm{KOH}, \mathrm{BnCl}$, Toluol, Rückfluss, 76\%; c) DiBAl-H, $\mathrm{CH}_{2} \mathrm{Cl}_{2},-7{ }^{\circ} \mathrm{C}, 66 \%$; d) TBDPSCl, Imidazol, $\mathrm{CH}_{2} \mathrm{Cl}_{2}, \mathrm{RT}, 99 \%$; e) $\mathrm{BCl}_{3}{ }^{\star} \mathrm{SMe}_{2}, \mathrm{CH}_{2} \mathrm{Cl}_{2}, \mathrm{RT}, 97 \%$.

Zum Aufbau der Seitenkette wurde der Alkohol 72 einer Swern Oxidation ${ }^{[32]}$ unterworfen. Mit Hilfe der Still-Gennari Variante ${ }^{[11]}$ der Horner-Wadsworth-Emmons Reaktion konnte dann die Z-Doppelbindung des $\alpha$. $\beta$-ungesättigten Methylesters 74 selektiv $(Z / E=20: 1)$ aufgebaut werden. Nach der Reduktion des Methylesters 74 
mit DiBAl-H zum entsprechenden Allylalkohol 75 wurde dieser durch eine Appel Reaktion $^{[33]}$ in das Allylbromid 76 überführt. Mit Tributylphosphin fand dann die Umsetzung zum Phosphoniumsalz 77 statt (Schema 17).
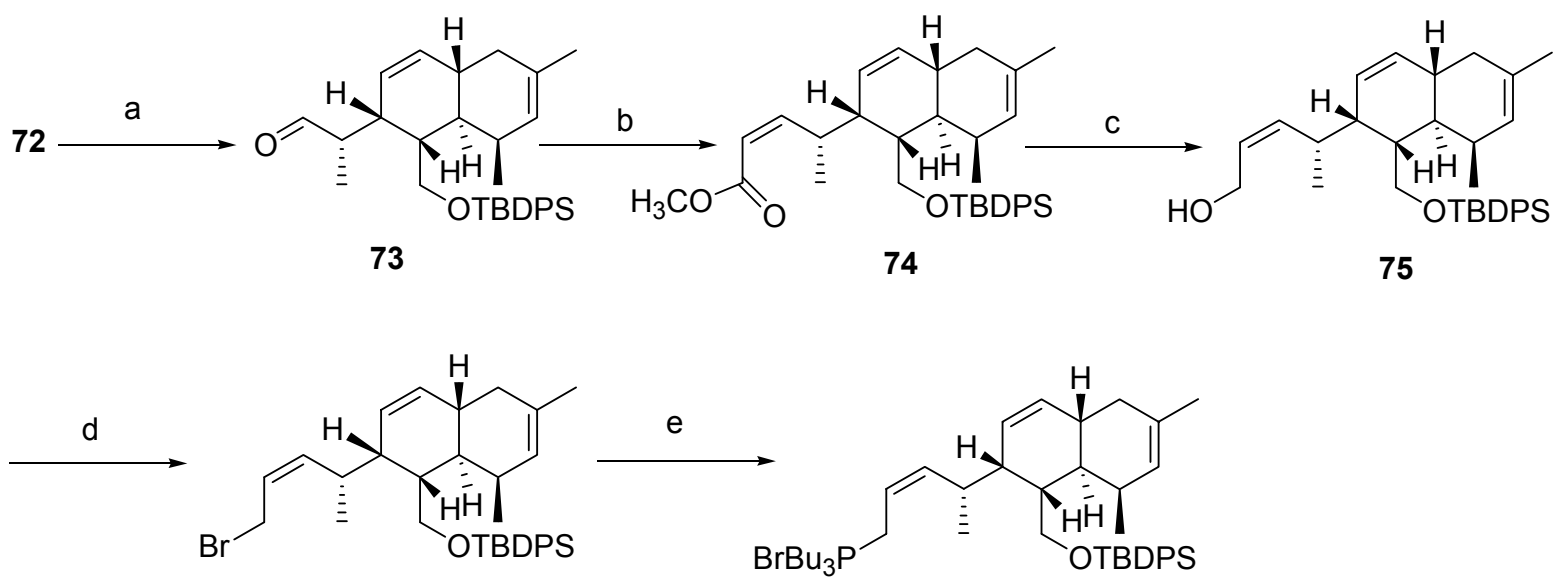

76

77

Schema 17 Darstellung des Phosphoniumsalzes 77.

Reaktionsbedingungen: a) Oxalylchlorid, DMSO, $\mathrm{Et}_{3} \mathrm{~N}, \mathrm{CH}_{2} \mathrm{Cl}_{2},-78{ }^{\circ} \mathrm{C} \rightarrow \mathrm{RT}, 81 \%$; b) $\mathrm{KHMDS}$, 18-Krone-6, $\left(\mathrm{CF}_{3} \mathrm{CH}_{2} \mathrm{O}\right)_{2} \mathrm{P}(\mathrm{O}) \mathrm{CH}_{2} \mathrm{COOCH}_{3}$, THF, $-40{ }^{\circ} \mathrm{C} \rightarrow-78{ }^{\circ} \mathrm{C}, 76 \%$; c) DiBAl-H, THF, $-50{ }^{\circ} \mathrm{C}$, $86 \%$; d) $\mathrm{CBr}_{4}, \mathrm{PPh}_{3}, \mathrm{MeCN}, \mathrm{RT}, 91 \%$; e) $\mathrm{PBu}_{3}, \mathrm{MeCN}, \mathrm{RT}$.

Der Kupplungspartner des Phosphoniumsalzes 77 für die Wittig Kupplung ${ }^{[12]}$ ist der Aldehyd 81. Dieser konnte über drei Schritte aus dem Milchsäureetylester (78) hergestellt werden (Schema 18). Zunächst wurde die Alkoholfunktion des Milchsäureesters (78) mit PMB-Trichloracetimidat unter Säurekatalyse geschützt und der resultierende Ester $\mathbf{7 9}$ mit DiBAl-H zum Alkohol $\mathbf{8 0}$ reduziert. Nach einer Swern Oxidation $^{[32]}$ erhielt man den Aldehyd 81.

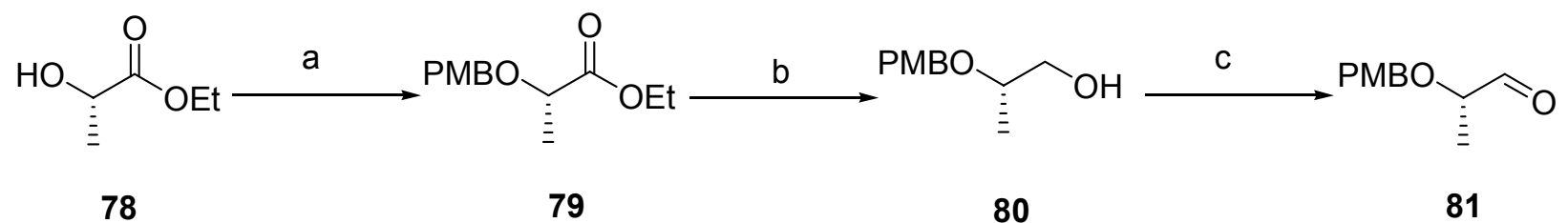

Schema 18 Darstellung des Aldehyds 81 für die Wittig Kupplung.

Reaktionsbedingungen: a) (4-Methoxybenzyl)-trichloracetimidat, $\mathrm{CSA}, \mathrm{CH}_{2} \mathrm{Cl}_{2}, \mathrm{RT}, 97 \%$; b) DiBAl-H, $\mathrm{CH}_{2} \mathrm{Cl}_{2},-78{ }^{\circ} \mathrm{C}, 86 \%$; c) Oxalylchlorid, DMSO, $\mathrm{Et}_{3} \mathrm{~N},-78^{\circ} \mathrm{C} \rightarrow \mathrm{RT}, 97 \%$.

Mit KOtBu wurde dann aus dem Phosphoniumsalz 77 in situ das Ylid generiert und anschließend mit dem Aldehyd 81 zur Reaktion gebracht. Dabei konnte die EDoppelbindung des Polyens 82 selektiv aufgebaut werden (E/Z-Selektivität > 20:1). 


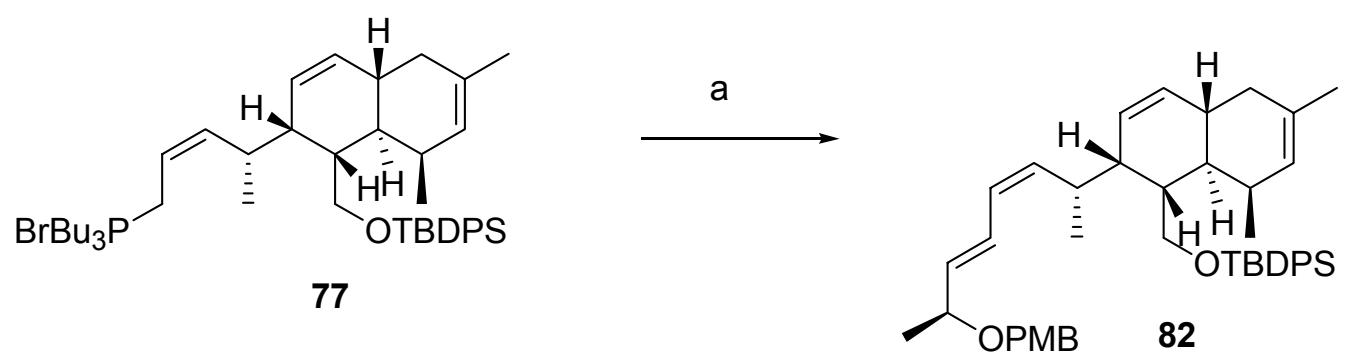

Schema 19 Wittig Kupplung von Aldehyd 81 und dem Phosphoniumsalz 77.

Reaktionsbedingungen: a) $81, \mathrm{KO}^{t} \mathrm{Bu}, \mathrm{THF} / \mathrm{Toluol}, \mathrm{RT}, 69 \%$.

Ingesamt sind zehn lineare Stufen notwendig, um ausgehend von Verbindung $\mathbf{6 6}$ das Dien-System aufzubauen. Die Synthesesequenz ist zwar insgesamt praktikabel, aber dennoch mit einem sehr hohen präparativen Aufwand verbunden. Daher wurde nach einer konvergenteren Strategie für diesen Abschnitt der Synthese gesucht.

Es gibt einige wenige Beispiele für vinyloge Still-Gennari Reaktionen von Roush ${ }^{[34]}$ und Evans ${ }^{[35]}$ (Schema 20). Diese Reaktion, angewendet auf die Synthese von Chlorotonil A, würde es ermöglichen, das E/Z-Dien in einem Schritt zu generieren.

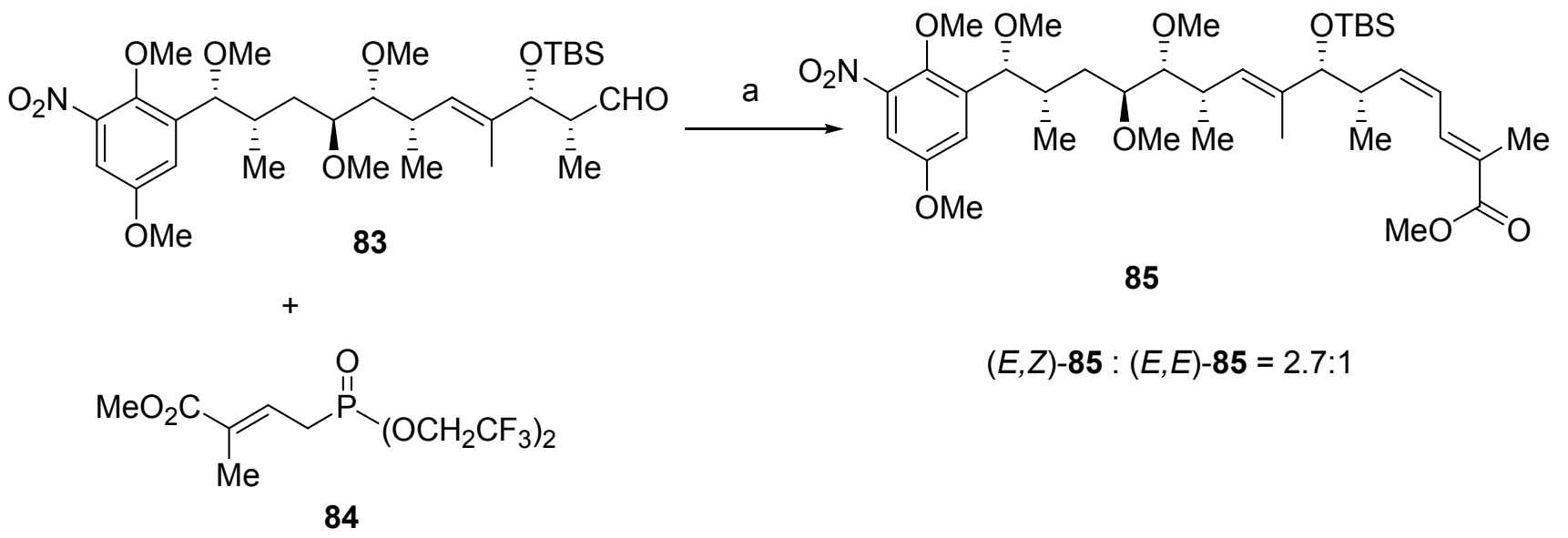

Schema 20 Vinyloge Still-Gennari Reaktion in der Totalsynthese von (+)-Macbecin von Evans.

Reaktionsbedingungen: a) $n$-BuLi, $\mathrm{E}_{2} \mathrm{O}, 70 \%$ des E/Z-Isomers.

Um diesen Weg zu erproben, wurde das für die vinyloge Still-Gennari Reaktion notwendige Phosphonat 89 dargestellt (Schema 21). 
<smiles>C=CCBr</smiles>

86<smiles>CCCCCC(C)OC=O</smiles>

81

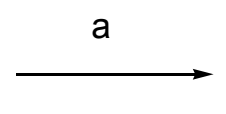

b

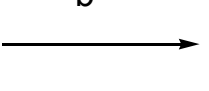

C

$87+88$<smiles>C=CC[PH](=O)CCCCC(F)(F)F</smiles>

87<smiles></smiles>

88

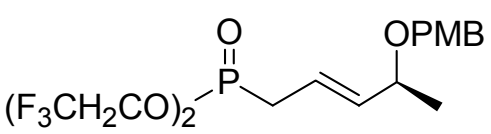

89

Schema 21 Darstellung des Phosphonates 89 für die vinyloge Still-Gennari Reaktion.

Reaktionsbedingungen: a) $\mathrm{P}\left(\mathrm{OCH}_{2} \mathrm{CF}_{3}\right)_{3}$, cat. $\mathrm{Bu}_{4} \mathrm{NI}, 170{ }^{\circ} \mathrm{C}, 61 \%$; b) $n$ - $\mathrm{BuLi},\left(\mathrm{Ph}_{3} \mathrm{PCH}_{3}\right)^{+} \mathrm{Br}^{-},-78^{\circ} \mathrm{C} \rightarrow$ RT, $73 \%$; c) Grubbs: zweiteGeneration, $\mathrm{CH}_{2} \mathrm{Cl}_{2}, 61 \%$.

Allylbromid (86) wurde mit Tris-(2,2,2-trifluorethyl)phosphit in einer Arbusov Reaktion $^{[36]}$ zum Phosphonat 87 umgesetzt. Der Aldehyd 81 konnte durch eine Wittig Reaktion in das Alken 88 überführt werden. Zusammen mit dem Phosphonat 87 erhielt man durch eine Kreuzmetathese das gewünschte Phosphonat 89 (Schema 21). Dieses weist an $C(4)$ ein Stereozentrum auf, das in Gegenwart von Base epimerisieren könnte. Bei der Wahl der Reaktionsbedingungen für die vinyloge StillGennari Reaktion musste dieses berücksichtigt werden (tiefe Temperaturen).

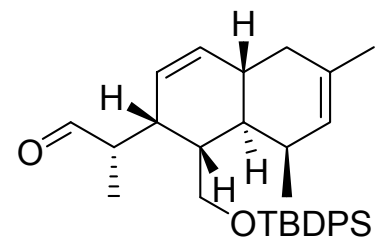

73 $\stackrel{\text { Reaktionsbedingungen }}{\longrightarrow}$

s. Tabelle 2

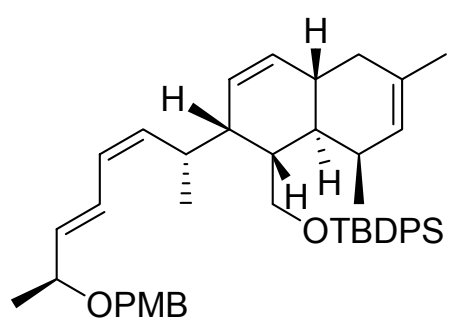

82<smiles>CC(C)=CC=CC[PH](=O)CCCCC(F)(F)F</smiles>

89

Schema 22 Vinyloge Still-Gennari Reaktion mit Phosphonat 89 und Aldehyd 73. 
Tabelle 2 Test verschiedener Reaktionsbedingungen bei der vinylogen Still-Gennari Reaktion.

\begin{tabular}{|c|c|c|c|}
\hline Eintrag & Reaktionsbedingungen & Z/E : E/E-Verhältnis & Ausbeute \\
\hline 1 & $\begin{array}{c}10 \text { eq } 89, \mathrm{KHMDS}, 18-\text { Krone-6, } \\
-40^{\circ} \mathrm{C}-\left(-78{ }^{\circ} \mathrm{C}\right), \mathrm{THF}\end{array}$ & $1: 2$ & $71 \%$ \\
\hline 2 & $\begin{array}{c}10 \text { eq } 89, \text { KHMDS, } \\
-78{ }^{\circ} \mathrm{C}, \mathrm{THF}\end{array}$ & $2: 1$ & $55 \%$ \\
\hline 3 & $\begin{array}{c}10 \text { eq } 89, \text { KHMDS, } \\
-80^{\circ} \mathrm{C}, \mathrm{Et}_{2} \mathrm{O}\end{array}$ & $3: 1$ & $55 \%$ \\
\hline 4 & $\begin{array}{c}10 \text { eq } 89, n \text {-BuLi, } \\
-78^{\circ} \mathrm{C}, \mathrm{Et}_{2} \mathrm{O}\end{array}$ & - & $\begin{array}{l}\text { Zersetzung des } \\
\text { Phosphonates }\end{array}$ \\
\hline
\end{tabular}

Es wurden verschiedene Reaktionsbedingungen bei der vinylogen Still-Gennari Reaktion getestet (Tabelle 2). Letztendlich wurden Bedingungen gefunden, unter denen das gewünschte Produkt $\mathbf{8 2}$ in mäßiger Ausbeute und Z-Selektivität dargestellt werden kann. Verglichen mit der schrittweisen Synthesestrategie wurden vier Stufen eingespart. 


\subsection{Aufbau der Triketon-Seitenkette}

Nach der Dien-Seitenkette sollte die Triketon-Seitenkette eingeführt werden. Dazu wurde die TBDPS-Schutzgruppe des Polyens 82 mit HF*Pyridin entfernt und der resultierende Alkohol $\mathbf{9 0}$ wurde mit Dess-Martin Periodinan zum Aldehyd 91 oxidiert. Anschließend war geplant durch eine Evans-Metternich-Aldol Reaktion, ${ }^{[13]}$ anschließende Oxidation des entstandenen Alkohols sowie Entfernung des EvansAuxilliars die Säure 93 zu generieren. Diese sollte nach Entfernung der PMBSchutzgruppe der Vorläufer des Macrolactons 94 sein (Schema 23).

82

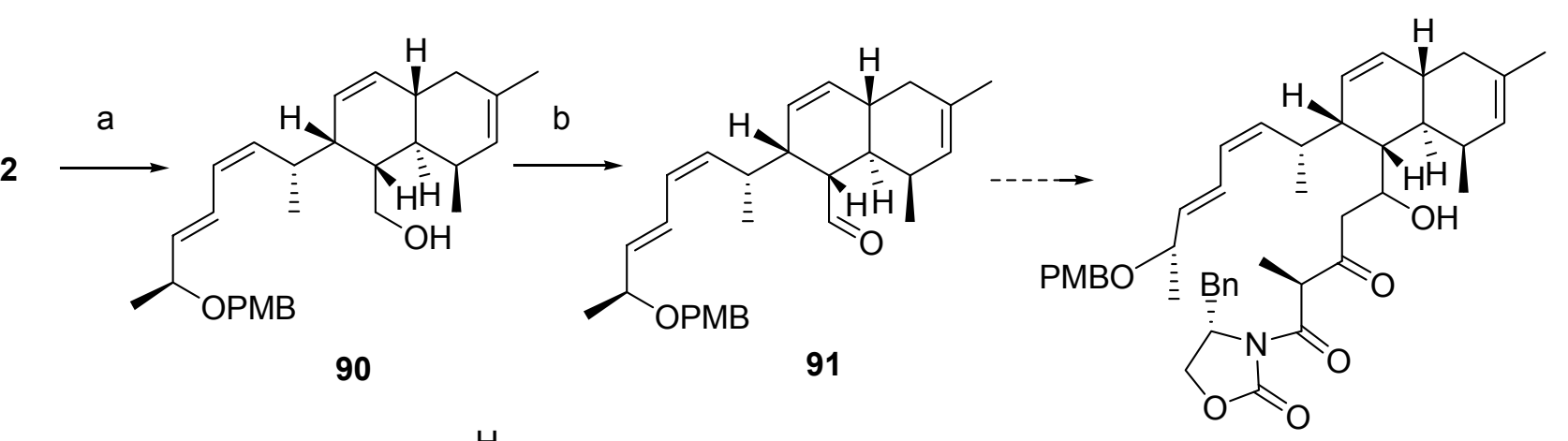

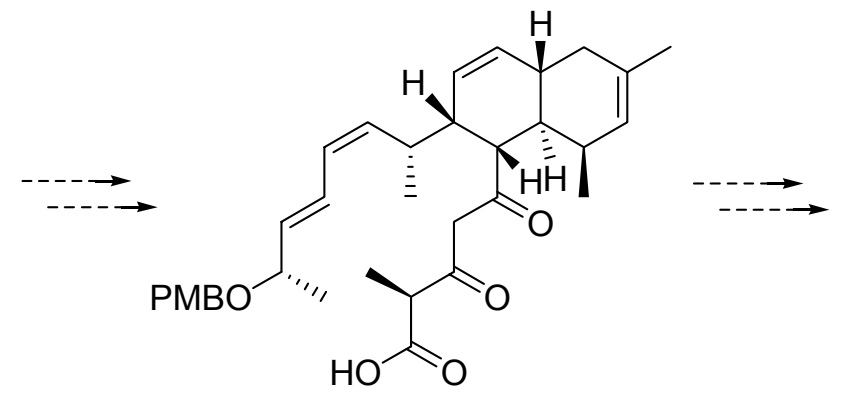

93

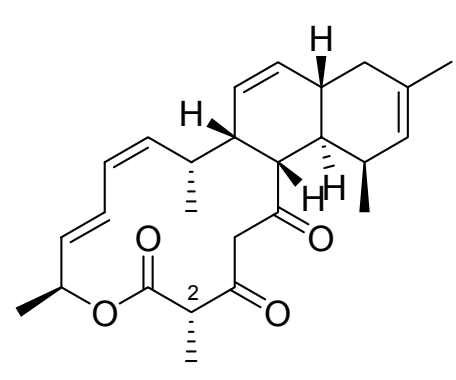

94

Schema 23 Aufbau des Tricarbonyl-Systems durch eine Evans-Metternich Aldol Reaktion.

Reaktionsbedingungen: a) HF*Pyridin, THF/Pyridin = 1:1, 63\%; b) Dess-Martin Periodinan, $\mathrm{CH}_{2} \mathrm{Cl}_{2}$, $58 \%$.

Die Evans-Metternich-Aldol Reaktion ermöglicht es, ein $\beta$-Keto-Imid mit einem Stereozentrum an $\mathrm{C}(2)$ ohne Epimerisierung an einen Aldehyd zu addieren. Je nach Wahl der Lewis-Säure kann ein all-syn oder ein anti-syn Produkt generiert werden (Schema 24). 


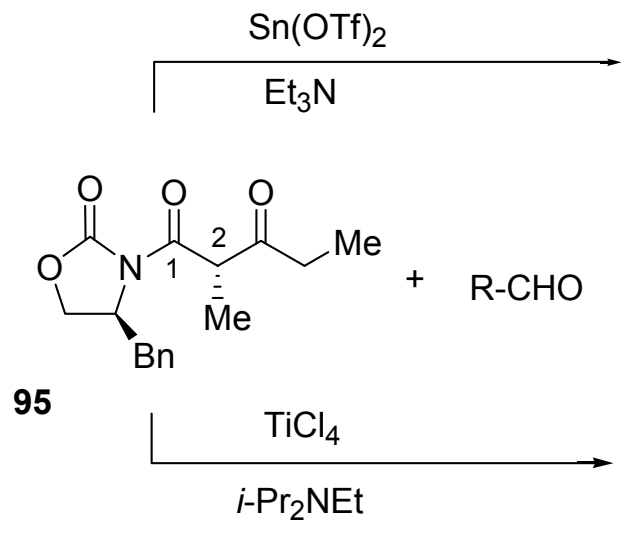<smiles>[R]C(O)[C@H](C)C(=O)[C@H](C)C(=O)N1C(=O)OC[C@@H]1Br</smiles>

$$
96 \quad \mathrm{R}=\text { Alkyl }
$$

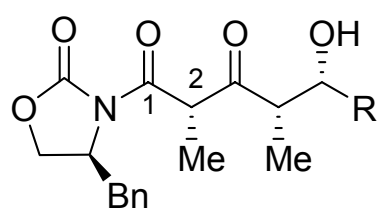

97

Schema 24 Evans-Metternich-Aldol Reaktion. ${ }^{[13]}$

Die Stabilität des Stereozentrums bzw. die geringe kinetische Acidität des Protons an $\mathrm{C}(2)$ resultiert aus einem Konformationseffekt, der das Wasserstoffatom an $\mathrm{C}(2)$ so ausrichtet, dass keine Überlappung mit der C(1)-Carbonyleinheit auftritt. Damit würde die bei einer Deprotonierung an $\mathrm{C}(2)$ entstehende negative Ladung nicht durch Resonanz stabilisiert werden. Die Ursache für den Konformationseffekt ist die Allylspannung zwischen der Methylgruppe an $\mathrm{C}(2)$ und dem sterisch anspruchsvollen Evans Auxilliar (Abbildung 14).

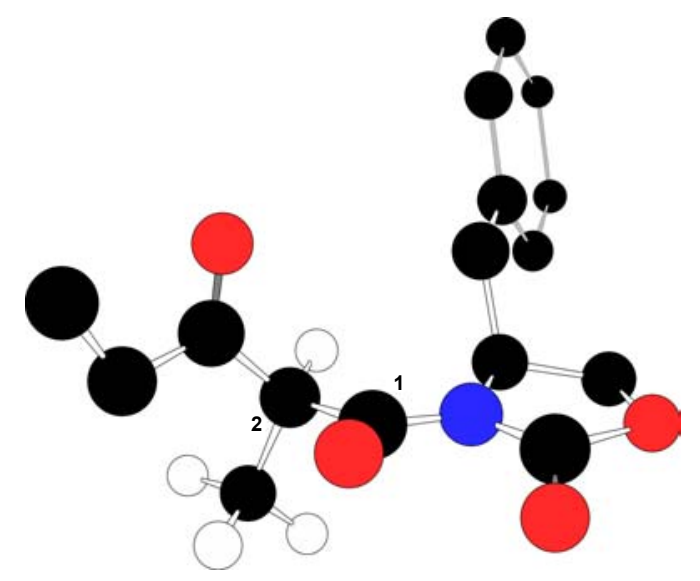

Abbildung 14 Dreidimensionale Darstellung des $\beta$-Ketoimids 95.

Für die Evans-Metternich-Aldol Reaktion musste zunächst das $\beta$-Ketoimid 100 hergestellt werden. ${ }^{[37]}$ Im ersten Schritt wurde Acetaldehyd mit Propionyloxazolidinon (+)-98 in einer Aldol Rektion umgesetzt. Das Ergebnis war das syn-Aldol Produkt 99. Nach einer Parikh-Döring Oxidation konnte das $\beta$-Ketoimid 100 isoliert werden (Schema 25). 
<smiles>CCC(=O)N1C(=O)OC[C@H]1Br</smiles>

98<smiles>C[C@H](C(=O)N1C(=O)OC[C@@H]1Br)[C@@H](C)O</smiles>

99

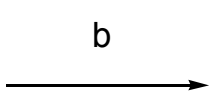<smiles>CC(=O)[C@H](C)C(=O)N1C(=O)OC[C@@H]1Br</smiles>

100

Schema 25 Darstellung von $\beta$-Ketoimid 100.

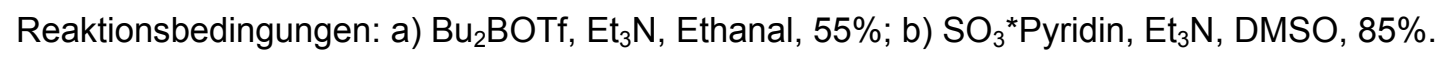

Die anschließende Umsetzung des $\beta$-Ketoimids 100 mit dem Aldehyd 91 in der Evans-Metternich-Aldol Reaktion war nicht erfolgreich. Die Reaktion wurde mit den Lewis-Säuren Zinntriflat und Titantetrachlorid getestet. Allerdings fand die Bildung von 92 unter den gewählten Reaktionsbedingungen nicht statt (Schema 26).<smiles>CC(=O)[C@H](C)C(=O)N1C(=O)OC[C@@H]1Br</smiles>

100<smiles>CC(C)=C(C)C</smiles>

91

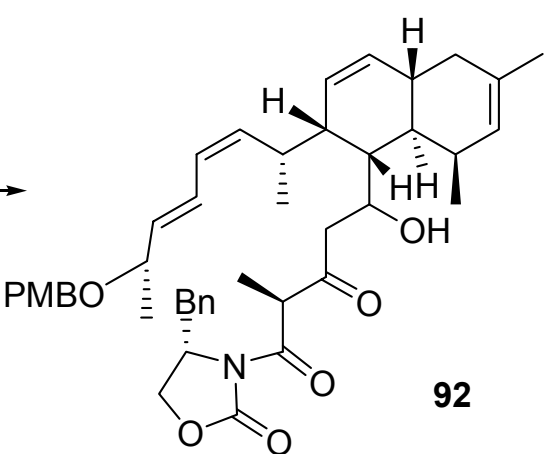

Schema 26 Evans-Metternich-Aldol Reaktion von $\beta$-Ketoimid 100 und Aldehyd 91.

Reaktionsbedingungen: a) 108, $\mathrm{TiCl}_{4},{ }^{i} \mathrm{Pr}_{2} \mathrm{NEt}$ oder $\mathrm{Sn}(\mathrm{OTf})_{2}, \mathrm{Et}_{3} \mathrm{~N} \mathrm{CH}_{2} \mathrm{Cl}_{2}$, kein Umsatz.

Der eleganteste Weg wäre, das Macrolacton zunächst als C(2)-Epimere 94 und Epi94 aufzubauen. Das Diastereomerengemisch soll nachträglich durch Epimerisierung des Stereozentrums an C(2) in die diastereomerenreine, gewünschte Verbindung 94 überführt werden (Abbildung 15).

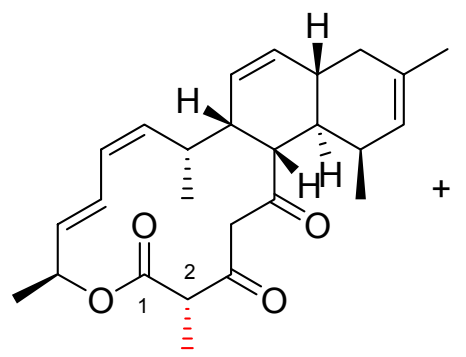

94

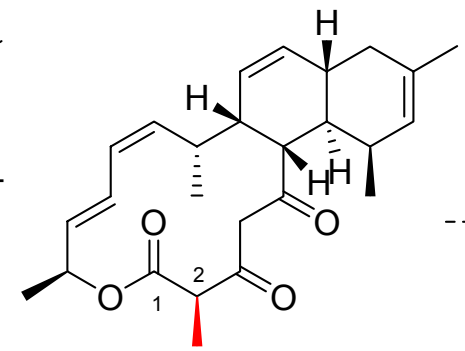

Epi-94

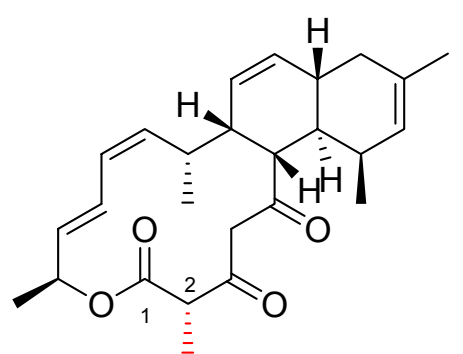

94

Abbildung 15 Diastereomerengemisch aus 94 und Epi-94. 
Mit der Kraftfeld Methode MM2* nach Allinger ${ }^{[38]}$ wurde für das Macrolacton 94 und Epi-94 eine Geometrieoptimierung durchgeführt. Dabei zeigte sich, dass 94 sowohl im Vakuum als auch in Toluol um $5 \mathrm{kcal} / \mathrm{mol}$ energieärmer ist als Epi-94. Das Macrolacton 94 sollte also das thermodynamische Produkt sein. Des Weiteren kann man erkennen, dass das Proton an $\mathrm{C}(2)$ bei 94 annähernd in einer Ebene mit der Carbonylgruppe der Esterfunktion liegt (Abbildung 16). Daher wird dieses Proton eine geringe kinetische Acidtiät zeigen. Bei Epi-94 hingegen steht das Proton an $\mathrm{C}(2)$ ungefähr senkrecht zur Carbonylgruppe der Esterfunktion und wird daher eine deutliche kinetische Acidität aufweisen. Daher sollte es möglich sein, Epi-94 unter basischen oder Lewis-sauren Bedingungen in das thermodynamisch günstigere Produkt 94 zu überführen.

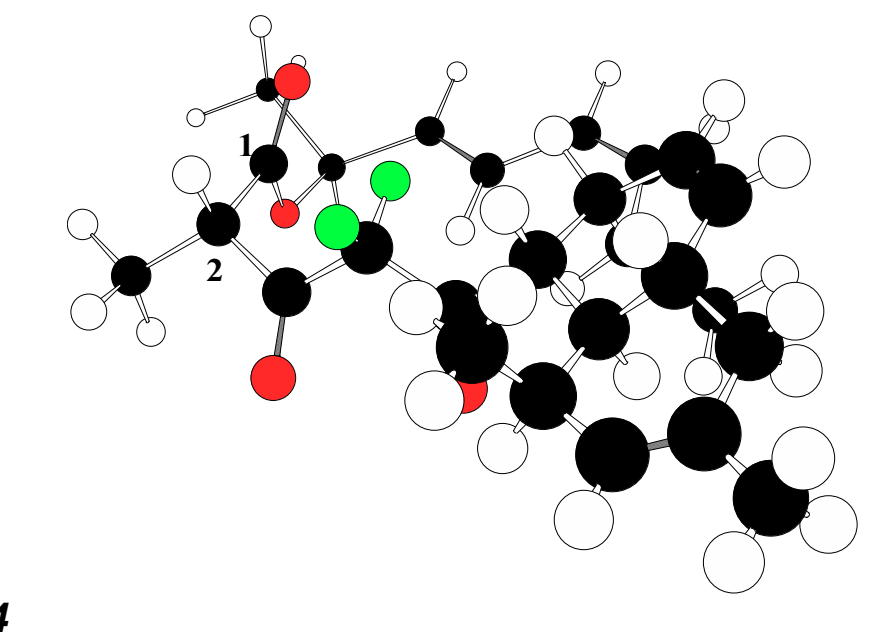

a) Macrolacton 94

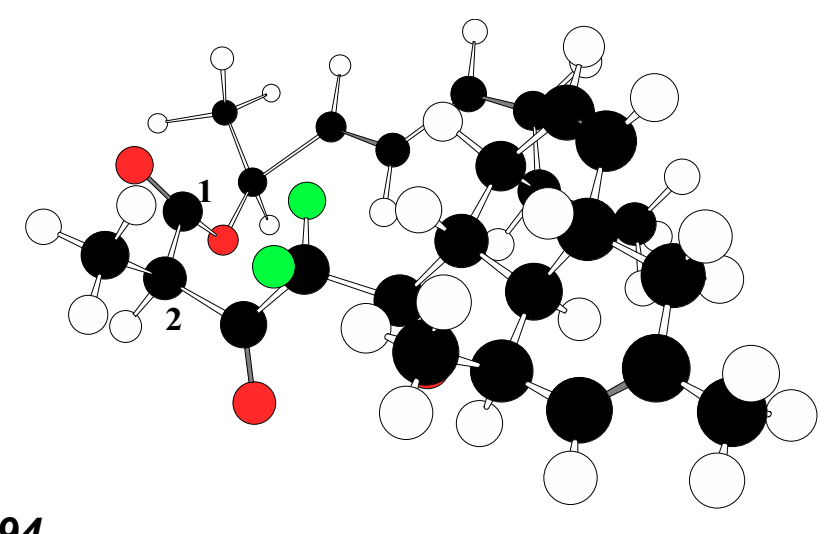

b) Macrolacton Epi-94

Abbildung 16 Geometrieoptimierung der Macrolactone 94 und Epi-94 mit MM2*.

Der Vorteil dieser Strategie war, dass eine größere Anzahl von Methoden zum Aufbau der Polyketon-Seitenkette in Frage kam. 
Eine Möglichkeit war die Roskamp Reaktion, ${ }^{[39]}$ bei der ein Aldehyd unter Katalyse von Zinn(II)chlorid mit Ethyldiazoacetat zu einem $\beta$-Ketoester umgesetzt wird (Schema 27).

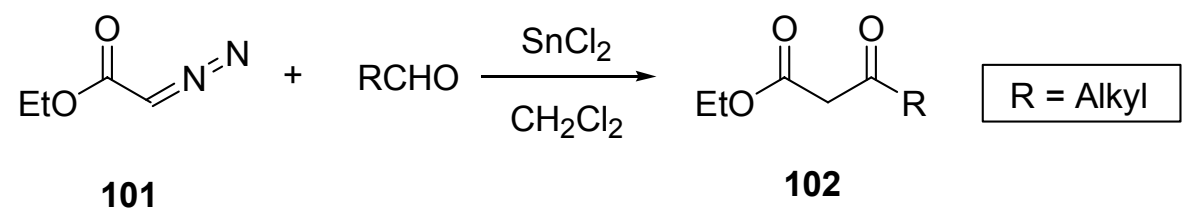

Schema 27 Die Roskamp Reaktion zum direkten Aufbau von $\beta$-Ketoestern aus Aldehyden mit Ethyldiazoacetat.

Um durch die Roskamp Reaktion eine Tricarbonylverbindung generieren zu können, musste der Diazoketoester 106 zusammen mit dem Aldehyd 91 umgesetzt werden (Schema 28). Der Diazoketoester 106 wurde in drei Stufen aus Diethylmethylmalonat (103) synthetisiert (Schema 28). Die anschließende Roskamp Reaktion war nicht erfolgreich. Unter den gewählten Reaktionsbedingungen konnte kein Produkt 107 isoliert werden. Die Verbindung 106 zersetzt sich unter Stickstofffreisetzung.<smiles>CCOC(=O)C(C)C(=O)OCC</smiles><smiles>[R]COC(=O)C(C)C(=O)CC([R])=O</smiles>

Schema 28 Roskamp Reaktion zur Darstellung der Tricarbonylverbindung 107.

Reaktionsbedingungen: a) $\mathrm{KOH}$, $\mathrm{EtOH}$ (abs.), $\mathrm{HCl}, 95 \%$; b) $\mathrm{SOCl}_{2}, 80{ }^{\circ} \mathrm{C}, 98 \%$; c) Diazomethan, Diethylether, 84\%; d) $\mathrm{SnCl}_{2}, \mathrm{CH}_{2} \mathrm{Cl}_{2}$, RT, Zersetzung.

Die nächste Reaktion, die zum Aufbau des Tricarbonylsystems in Betracht gezogen wurde, war die Aldolreaktion des Aldehyds 91 mit dem Dianion von Ethyl-2methylacetoacetat (109), das durch Deprotonieren mit NaH und $n$-BuLi generiert wurde. ${ }^{[40]}$ 
In verschiedenen Testansätzen wurde die Durchführbarkeit dieser Reaktion überprüft. Dabei konnte nicht nur der Aldehyd 108 erfolgreich umgesetzt werden, sondern auch der entsprechende Methylester 111. Der Vorteil bei der Addition des Dianions von (109) an den Methylester 111 war, dass das erhaltene Produkt bereits die gewünschte zweite Oxo-Funktion enthält und so der sonst noch notwendige Oxidationschritt entfällt (Schema 29).<smiles>CC1=C[C@H](C)[C@]2(C)C(C=O)[C@H]([C@@H](C)COc3ccccc3)C=C[C@H]2C1</smiles>

108<smiles>COC(=O)C1C([C@H](C)COCc2ccccc2)C=C[C@H]2CC(C)=C[C@H](C)[C@H]12</smiles>

111
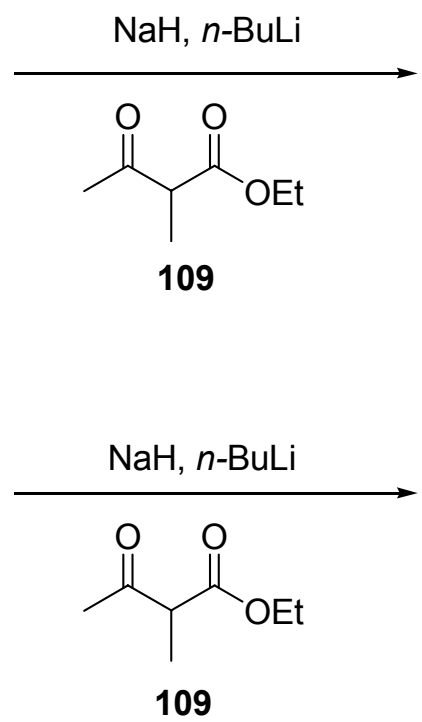

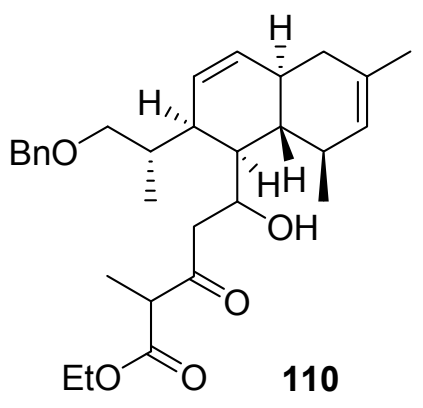

$65 \%$<smiles>[CH][C@@]12C(C(=O)CC(=O)C(C)C(=O)OCC)C([C@H](C)COCc3ccccc3)C=C[C@H]1CC(C)=C[C@@H]2C</smiles>

Schema 29 Ethyl-2-methylacetoacetat als Synthesebaustein für den Aufbau der Tricarbonylstruktur.

Die Syntheseroute zu 107 (Schema 30) könnte wesentlich effizienter gestaltet werden, wenn man nach der Öffnung von 66 die entstehende Säure 7 nicht reduziert und mit einer Schutzgruppe versieht, sondern die Säure direkt in den Methylester überführt. Frühere Versuche hatten gezeigt, dass es problematisch ist, den Methylester zu isolieren, da stets das Lacton 66 zurückgebildet wurde. Daher wurde die Durchführung so modifiziert, dass nach der Öffnung des Lactons 66 die Säurefunktion in situ in den Methylester überführt und die Alkoholfunktion in situ oxidiert wurde. Damit war die Bildung von Lacton 66 nicht mehr möglich und man konnte den Aldehyd 113 isolieren, der in die vinylogen Still-Gennari Reaktion eingesetzt wurde. Die Addition des Dianions von (109) an den Methylester 114 konnte erfolgreich durchgeführt werden (Schema 30). Allerdings war eine Isolierung von 107 nicht möglich, da Ethyl-2-methylacetoacetat (109) in 10-20-fachem Überschuss eingesetzt wurde und (109) sowie das Reaktionsprodukt 107 einen 
annährend gleichen $R_{f}$-Wert aufweisen. Da die Mischung selbst per HPLC nicht getrennt werden konnte, wurde sie ohne weitere Reinigung in der nächsten Reaktion eingesetzt.<smiles>COC(=O)[C@]1(C)[C@@H](C)C=C[C@H]2CC(C)=C[C@H](C)[C@H]2[C@H]1C=O</smiles>

66

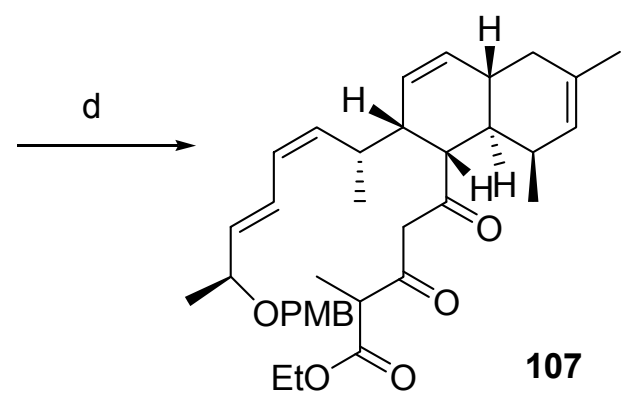

113

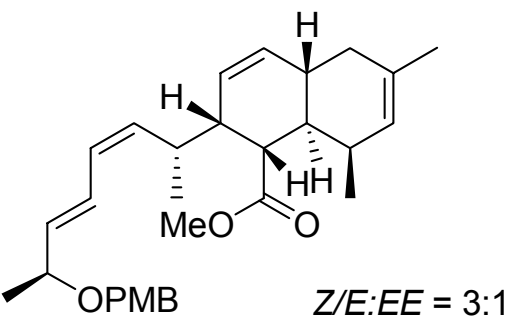

114

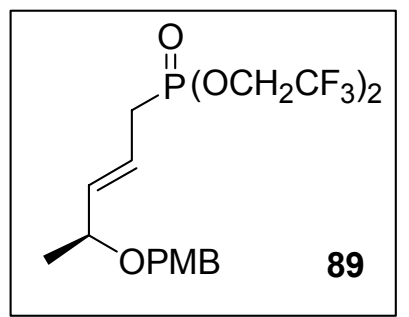

Schema 30 Stark verkürzte Route zur Synthese von 107.

Reaktionsbedingungen: a) $\mathrm{KOH}, \mathrm{MeOH}$; dann Diazomethan; b )Dess-Martin-Periodinan, $77 \%$ über drei Stufen; c) KHMDS, 89, 55\%; d) NaH, $n$-BuLi, Ethyl-2-methylacetoacetat. 


\subsection{Macrolactonisierung und abschließende Halogenierung}

Zur Bildung des Macrolactons 94 aus 107 musste zunächst die PMB-Schutzgruppe entfernt werden. Dieses ist prinzipiell mit Oxidationsmitteln oder mit Lewis-Säuren möglich. Zuerst wurde die Reaktion mit DDQ getestet, was zur Zersetzung von 107 führte (Schema 31).

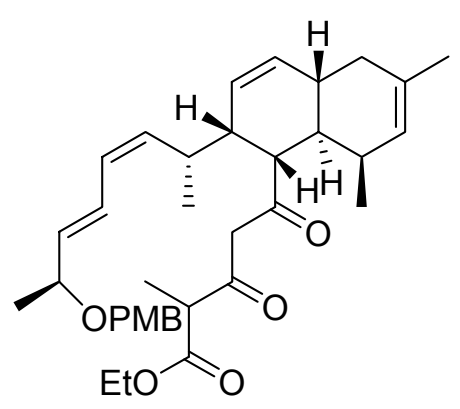

107

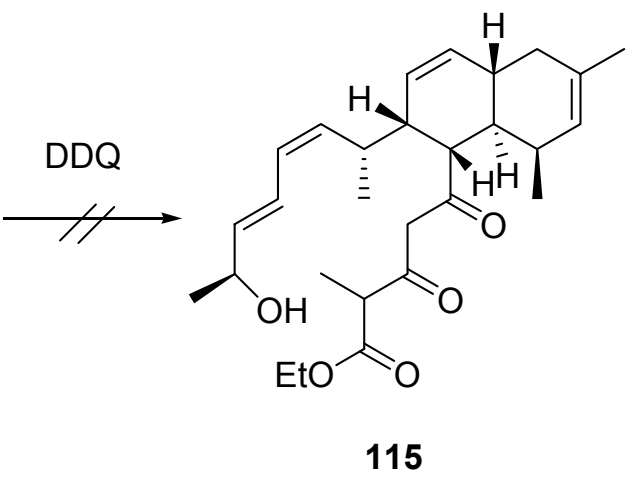

115

\section{Schema 31 Entfernung der PMB-Schutzgruppe.}

Das Gemisch aus PMB-Ether 107 und Ethyl-2-methylacetoacetat (109) wurde deshalb in Toluol gelöst und dann mit wenigen Tropfen $\mathrm{BF}_{3}{ }^{*} \mathrm{OEt}_{2}$ versetzt. Unter diesen Bedingungen war nach wenigen Minuten das gesamte Startmaterial verbraucht. Die Analyse des gereinigten Reaktionsprodukts zeigte, dass unter Einfluss der Lewis-Säure $\mathrm{BF}_{3}{ }^{*} \mathrm{OEt}_{2}$ nicht nur die Schutzgruppe abgespalten wurde, sondern auch die Macrolactonisierung von 107 erfolgt war. Es wurde nur das Produkt 94 isoliert. Die Epimerisierung von Epi-94 zu 94 fand also ebenfalls unter den Lewissauren Bedingungen statt. Aus dem Diastereonerengemisch hatte sich, vermutlich durch den Einfluss von $\mathrm{BF}_{3}{ }^{*} \mathrm{OEt}_{2}$ über Keto-Enol-Tautomerie, das thermodynamisch stabilere Produkt 94 gebildet. (Schema 32).

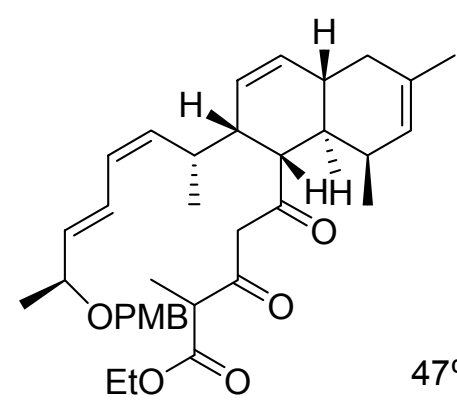

107<smiles>CC1=C[C@H](C)[C@@H]2[C@@H](C)C=C[C@H]([C@@H](C)/C=C\C=C/C(C)OC(=O)[C@H](C)C(=O)CC(=O)[C@H]2C)[C@H]1C</smiles>

94

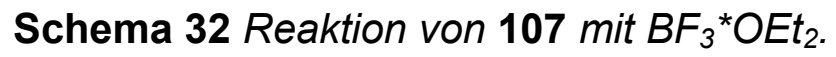


Der letzte Schritt, die Einführung der beiden geminalen Chlor-Substituenten im Macrolacton 94 wurde mit N-Chlorsuccinimid unter basischen Bedingungen erzielt. ${ }^{[42]}$ Damit konnte die Synthese von Chlorotonil A erfolgreich abgeschlossen werden (Schema 33).

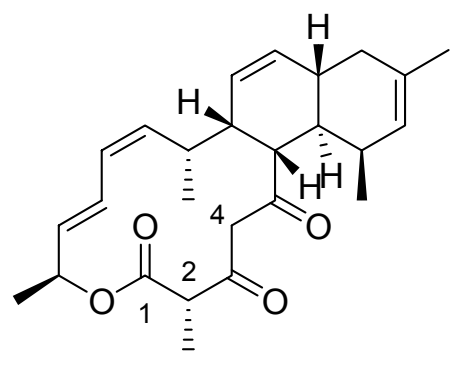

94
NCS, 2,6-Lutidin

$65 \%$

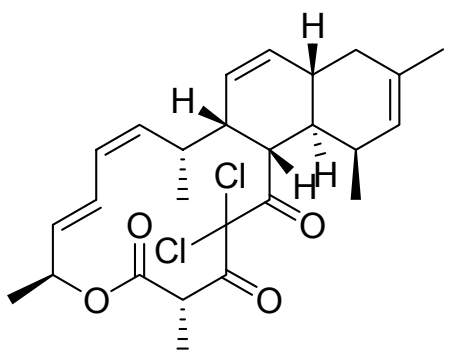

Chlorotonil A (3)

Schema 33 Chlorierung des Macrolactons 94.

Darüber hinaus konnte mit N-Bromsuccinimid die entsprechende bromierte Verbindung 116 hergestellt werden - Bromotonil (Schema 34).

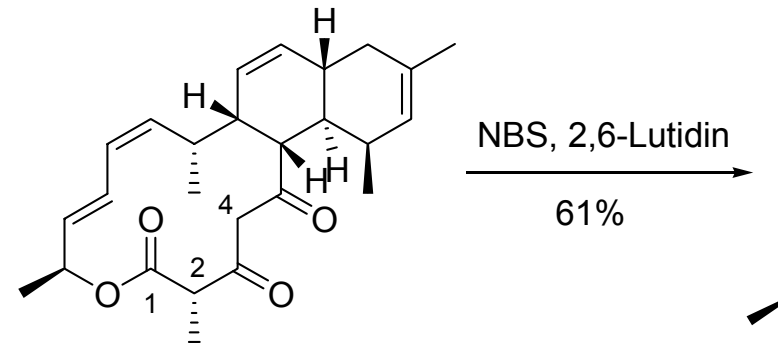

94

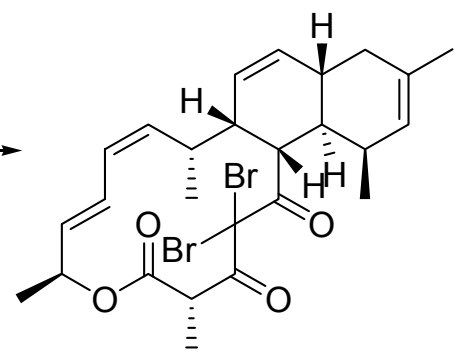

116

Schema 34 Darstellung von Bromotonil 116.

In beiden Fällen konnte beobachtet werden, dass Nebenprodukte auftreten, bei denen es sich wahrscheinlich um dreifach halogenierte Verbindungen handelte, da das Proton an $\mathrm{C}(2)$ ebenfalls leicht durch ein Halogenatom ausgetauscht werden kann. Wahrscheinlich ist die Enolform die Spezies, die halogeniert wird. Im ${ }^{1} \mathrm{HNMR}$ Spektrum erkennt man deutlich, dass die Diketo-Einheit von 94 in Lösung vollständig enolisiert vorliegt während die Säure-Einheit nicht dazu neigt. Daher ist zu erwarten, dass zuerst die Positionen an $\mathrm{C}(4)$ halogeniert werden und bei einem Überschuss von NCS oder NBS die Position an C(2). 


\subsection{Dehalogenierung von Chlorotonil A}

Vor dem eigentlichen Beginn der Synthese von Chlorotonil A wurde getestet, ob der geplante letzte Schritt der Synthese, die Chlorierung des Macrolactons 94, möglich ist. Dazu wurde isoliertes Chlorotonil A (3) mit Samariumdiiodid behandelt (Schema 35). ${ }^{[41]}$ Unter diesen Reaktionsbedingungen konnten die Chlor-Substituenten aus dem Molekül entfernt werden.

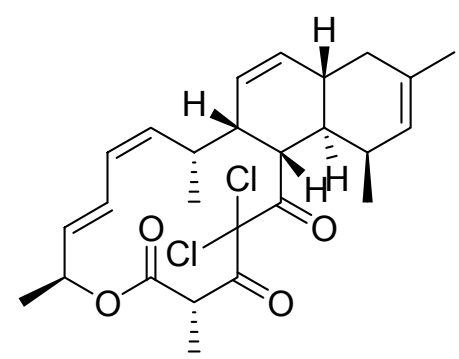

Chlorotonil A (3)

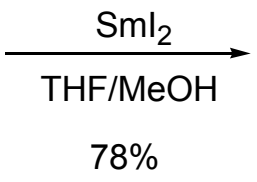

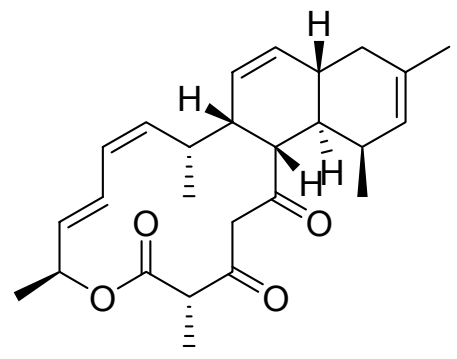

94

Schema 35 Dehalogenierung von Chlorotonil A (3).

Die Reaktion verläuft über einen radikalischen Mechanismus (Schema 36), wobei pro Chlor-Substituent zwei Äquivalente Samariumdiiodid notwendig sind. Bei einem Überschuss an Samariumdiiodid werden die Carbonylgruppen reduziert. Die Esterfunktion ist unter den gegebenen Reaktionsbedingungen stabil und wird nur bei zusätzlicher Zugabe von HMPA reduziert.<smiles>[R]C(=O)C(Cl)(Cl)C(=O)[R]([H])=CO</smiles>
117<smiles>[R]C(=O)C(Cl)=C([R])O</smiles>

120<smiles>[R]C(=O)C(Cl)(Cl)C([R])O</smiles>

118

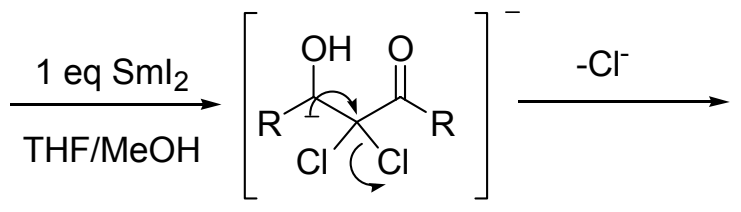

119

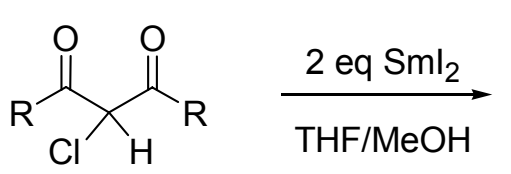

121<smiles>[R]C(=O)C([2H])C([R])=O</smiles>

122

$$
\mathrm{R}=\mathrm{Alkyl}
$$

Schema 36 Mechanismus der Samariumdiiodid vermittelten Abspaltung der ChlorSubstituenten. 
Mit dem hergestellten Macrolacton 94 wurden dann verschiedene Methoden zur Chlorierung getestet, wobei sich NCS mit 2,6-Lutidin als die beste herausstellte (Kapitel 3.8). Damit war sichergestellt, dass die Synthese nicht am letzten Schritt scheitern würde. Außerdem konnte das nach der Macrolactonisierung mit $\mathrm{BF}_{3}{ }^{*} \mathrm{OEt}_{2}$ entstandene Produkt durch den Vergleich der ${ }^{1} \mathrm{HNMR}$ als Lacton 94 und vor allem als das gewünschte Diastereomer identifiziert werden. 


\subsection{Zusammenfassung und Ausblick}

Chlorotonil A (3) konnte erfolgreich durch eine stereoselektive Synthese mit 21 linearen Stufen und einer Gesamtausbeute von 1.5\% dargestellt werden. Des Weiteren konnte die absolute Konfiguration, welche zuvor über Röntgenstrukturanalyse bestimmt wurde, bestätigt werden. Zugleich wurden einige interessante Reaktionen ausgearbeitet, die auch in andere Naturstoffsynthesen Anwendung finden können, wie z.B. die Kaskadenreation zur Bildung des Diels-Alder Produktes und die Überführung eines Diastereomerengemisches in die thermodynamisch günstigste diastereomerenreine Verbindung durch Keto-EnolTautomerie.

Da der Naturstoff Chlorotonil A(3) eine schlechte Wasserlöslichkeit aufweist, ist sein pharmakologisches Profil für einen Wirkstoffkandidaten wenig geeignet. Wichtig wäre es daher, von Chlorotonil A (3) Derivate zu erzeugen, die wasserlöslicher sind als der Naturstoff selber. Eine Möglichkeit ist die Einführung von Hydroxyfunktionen. Dabei bietet es sich an, die Doppelbindungen teilweise zu dihydroxylieren oder zu hydroborieren/oxidieren. Auch durch Epoxidierung und anschließende Epoxidöffnung oder durch Reduktion der Carbonylfunktionen könnte man ausgehend von Chlorotonil A (3) leicht an wasserlöslichere Derivate gelangen. Allerdings könnte die Selektivität dieser Reaktionen ein Problem darstellen. Da es sowohl mehrere Doppelbindungen als auch Carbonylgruppen im Molekül gibt, sind diese Methoden nur geeignet, wenn selektiv ein Produkt erzeugt werden kann oder zumindest die Produktgemische getrennt werden können.

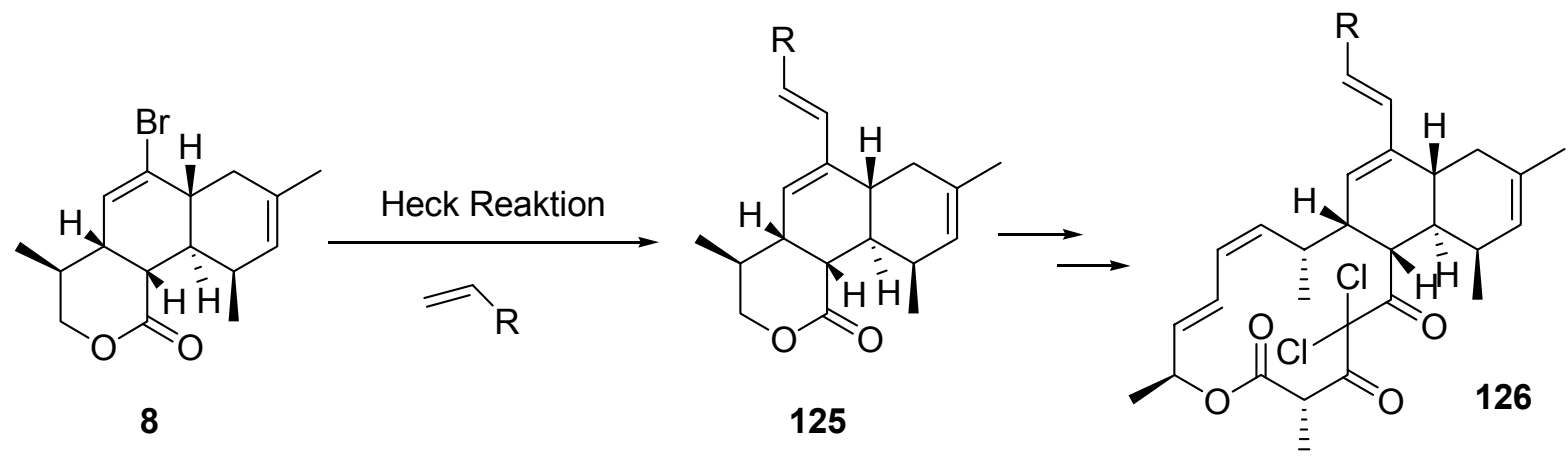

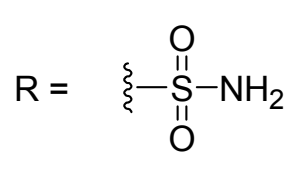

123<smiles>CCO[C@H]1O[C@H](COC(C)=O)[C@@H](OC2CC2)[C@H](OC(C)=O)[C@@H]1OC(C)=O</smiles>

124

Schema 37a Heck Reaktion zur Darstellung wasserlöslicherer Derivate. 
Ein anderer Ansatz ist, Derivate über Totalsynthese darzustellen. Zum Beispiel könnte das Diels-Alder Produkt 8 über den Brom-Substituenten durch eine Heck Reaktion $^{[44,45,46]}$ mit verschiedenen Alkenen gekuppelt werden, die polare funktionelle Gruppen aufweisen. ${ }^{[43,47]}$ Anschließend könnte die Synthese, wie in dieser Arbeit beschrieben, bis zum Ende durchgeführt werden (Schema 37a).

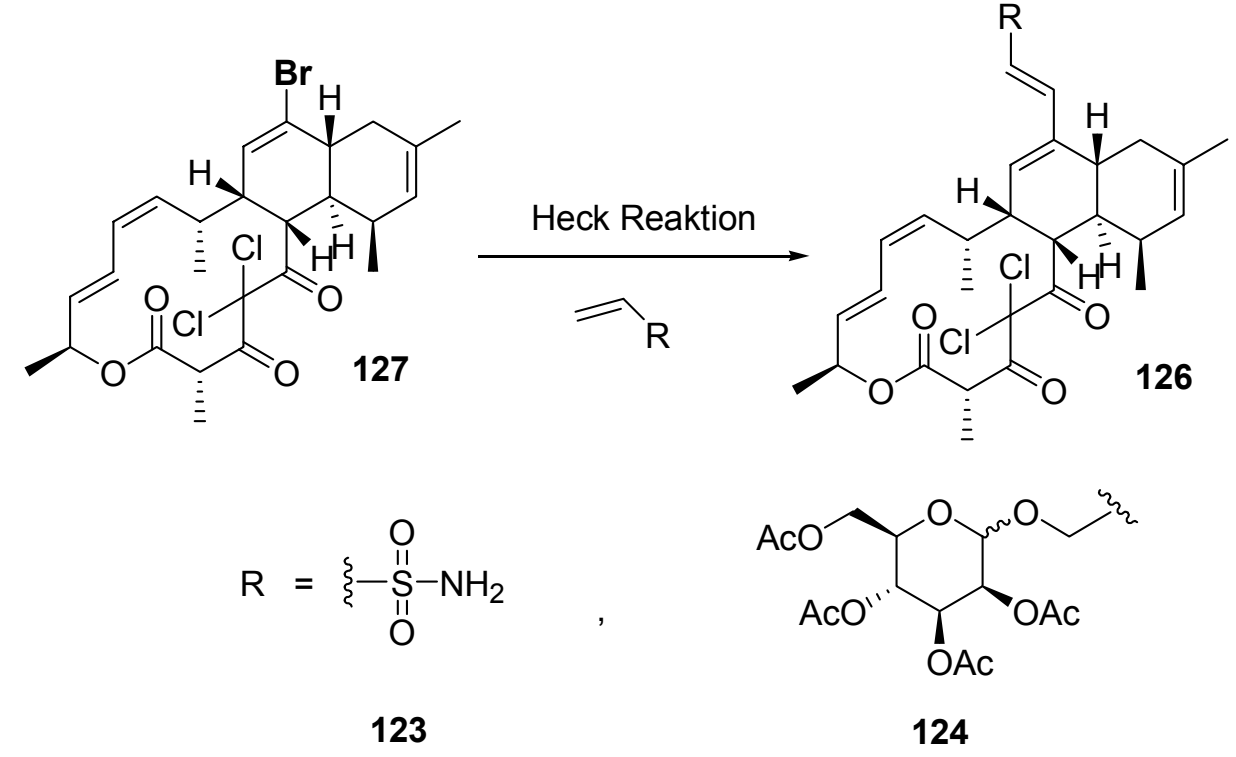

Schema 37b Heck Reaktion zur Darstellung wasserlöslicherer Derivate.

Eine andere, vielleicht einfachere Möglichkeit ist, den Brom-Substituenten nicht zu entfernen und für eine Derivatisierung durch eine Heck Reaktion aufzuheben. Im Schema $37 \mathrm{~b}$ sind zwei Alkene mit polaren funktionellen Gruppen aufgeführt. Während der Heck Reaktion müssen die $\mathrm{OH}-F u n k t i o n e n$ des Zuckers geschützt sein. Am Ende der Synthese können sie jedoch durch entsprechende Reagenzien frei gesetzt werden.

Mit Hilfe der erzeugten Derivate könnten dann Struktur-Aktivitätsbeziehungen aufgeklärt werden. Erste Ergebnisse liegen bereits für die vorhandenen Analoga 94 und 116 vor. Die geminalen Chloride haben keinen Einfluss auf die Aktivität. Sowohl der dehalogenierte als auch der bromierte Naturstoff weist antibiotische Aktivität auf. 


\section{Experimenteller Teil}

\subsection{Allgemeine Bemerkungen}

1H-NMR-Spektren wurden mit den Geräten WP-200 SY und AM-400 der Firma Bruker gemessen. Als Lösungsmittel wurde, sofern nicht anders angegeben, Deuterochloroform $\left(\mathrm{CDCl}_{3}\right)$ verwendet. Die chemischen Verschiebungen sind in ppm auf der $\delta$-Skala angegeben. Als interner Standard diente dabei das $\mathrm{CHCl}_{3}$-Signal bei $\delta=7.24$ ppm. Die Kopplungskonstanten sind in Hertz $(\mathrm{Hz})$ aufgeführt. Die Signalmultiplizitäten sind wie folgt gekennzeichnet:

$\mathrm{s}=$ Singulett, $\mathrm{d}=$ Dublett, $\mathrm{t}=$ Triplett, $\mathrm{q}=$ Quartett, qui $=$ Quintett, $\mathrm{m}=$ Multiplett, $\mathrm{dd}=$ Doppeldublett, $\mathrm{dt}=$ Doppeltriplett, $\mathrm{dq}=$ Doppelquartett, $\mathrm{br}=$ breit.

13C-NMR-Spektren wurden bei 100 MHz mit dem AM-400 der Firma Bruker und dem $\mathrm{CDCl}_{3}$-Signal bei $\delta=77.0 \mathrm{ppm}$ als internem Standard gemessen. Als Lösungsmittel diente, sofern nicht anders angegeben, $\mathrm{CDCl}_{3}$. Die Spektren sind nach dem APT oder DEPT - Verfahren erstellt worden.

Massenspektren (MS, HRMS) wurden mit dem Gerät Autospec von VG bei einem lonisierungspotential von $70 \mathrm{eV}$ aufgenommen.

Drehwerte $[\alpha]$ wurden mit dem Polarimeter Perkin-Elmer 341 gemessen. Die verwendete Wellenlänge, die Temperatur, das Lösungsmittel und die Konzentration (in $10 \mathrm{mg} / \mathrm{ml}$ ) der Meßsubstanz sind angegeben.

Gaschromatogramme sind mit einem HP 6890-II der Fa. Hewlett-Packard mit einer SE-54-Kapillarsäule (25 m, Fa. Macherey-Nagel) und Flammenionisator gemessen worden, wobei Stickstoff als Trägergas diente. Chirale Gaschromatogramme wurden mit einem HP 5890-II der Fa. Hewlett-Packard und einer chiralen Säule (Lipodex E Nr. 723368, Oktakis-(2,6-di-O-pentyl-O-butyryl)- $\gamma$-cyclodextrin als stationäre Phase) der Fa. Macherey-Nagel aufgenommen. 
Säulenchromatographie wurde unter Verwendung von Silicagel (Korngröße 40-60 $\mu \mathrm{m}$, Porendurchmesser 60Å) der Firma J.T. Baker bei leichtem Überdruck durchgeführt.

Analytische Dünnschichtchromatographie erfolgte auf mit Kieselgel beschichteten Aluminiumfolien $60 \mathrm{~F}_{254}$ (Schichtdicke $0.2 \mathrm{~mm}$ ) der Firma Merck. Als Färbereagentien wurden Vanillin-, Kaliumpermanganat- oder Cer-Lösungen verwendet.

Lösungsmittel sind nur destilliert eingesetzt worden. Die absoluten Lösungsmittel Dichlormethan, Tetrahydrofuran, Diethylether, N,N-Dimethylformamid und Toluol wurden einem Solvent Purification System (MB SPS) der Firma Braun entnommen.

Triethylamin und Diisopropylethylamin wurden über Calciumhydrid refluxiert und abdestilliert.

Acetonitril, Methanol, Ethanol und Pyridin wurden von Acros oder Aldrich kommerziell erworben und wie erhalten eingesetzt.

Reaktionen wurden unter Argonatmosphäre durchgeführt. Bei allen Experimenten wurde, sofern nicht anders angegeben, ein Magnetrührer verwendet. 


\subsection{Beschreibung der Versuche}

Darstellung von Methylester $31^{[18]}$

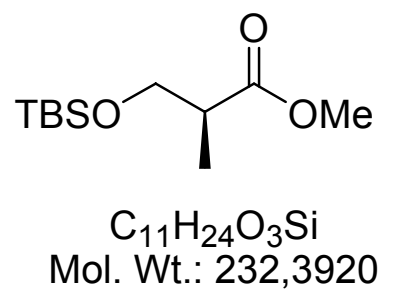

31

Bei Raumtemperatur wird zu (+)-Methyl-L- $\beta$-hydroxyisobutyrat (30) (100 mg, $0.84 \mathrm{mmol})$ in $\mathrm{CH}_{2} \mathrm{Cl}_{2}(1.5 \mathrm{~mL})$ nacheinander DIPEA (164 mg, $\left.1.27 \mathrm{mmol}\right)$, DMAP (80 mg, $0.66 \mathrm{mmol})$ und TBSCl (166 mg, $1.10 \mathrm{mmol}$ ) hinzugefügt. Nach eineinhalb Stunden wird die Reaktionsmischung durch Zugabe von Hexan (10 mL) gequencht. Dann wird mit Phosphatpuffer pH $7(2 \times 5 \mathrm{~mL})$ gewaschen und mit Hexan $(2 \times 5 \mathrm{~mL})$ extrahiert. Die gesammelten organischen Phasen wäscht man erneut mit Phosphatpuffer pH 7 (2 x $5 \mathrm{~mL})$ und anschließend mit gesättigter, wässriger $\mathrm{NaCl}$ Lösung ( 1 x $5 \mathrm{~mL})$. Dann werden sie mit $\mathrm{MgSO}_{4}$ getrocknet. Der Feststoff wird abfiltriert und das Lösungsmittel anschließend am Rotationsverdampfer entfernt. Nach säulenchromatographischer Reinigung (Hexan/EtOAc $=10: 1$ ) erhält man das gewünschte Produkt 31 (188 mg, $0.81 \mathrm{mmol}, 96 \%)$ als farbloses Öl: ${ }^{1} \mathrm{H}-\mathrm{NMR}$ $\left(400 \mathrm{MHz}, \mathrm{CDCl}_{3}\right) \delta 3.77$ (dd, $\left.J=9.7,6.9 \mathrm{~Hz}, 1 \mathrm{H}\right), 3.67(\mathrm{~s}, 3 \mathrm{H}), 3.64$ (dd, $J=9.7$, $6.0 \mathrm{~Hz}, 1 \mathrm{H}), 2.69-2.59(\mathrm{~m}, 1 \mathrm{H}), 1.13(\mathrm{~d}, J=7.0 \mathrm{~Hz}, 3 \mathrm{H}), 0.86(\mathrm{~s}, 9 \mathrm{H}), 0.03(\mathrm{~s}, 3 \mathrm{H})$, 0.027 (s, 3H); ${ }^{13} \mathrm{C}-\mathrm{NMR}\left(100 \mathrm{MHz}, \mathrm{CDCl}_{3}\right) \quad \delta$ 175.46, 65.23, 51.48, 42.52, 25.76, 18.19, 13.44, -5.52; HRMS berechnet für $\mathrm{C}_{7} \mathrm{H}_{15} \mathrm{O}_{3} \mathrm{Si}_{1}\left(\mathrm{C}_{11} \mathrm{H}_{24} \mathrm{O}_{3} \mathrm{Si}_{1}\right.$ minus $t$-Butyl): 175.1495, gefunden 175.1495. 


\section{Darstellung von Alkohol 32 $^{[18]}$}

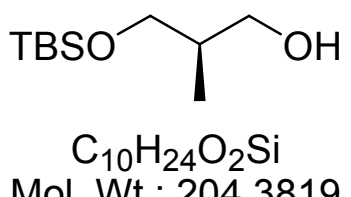

32

Zu einer Lösung $\left(-78{ }^{\circ} \mathrm{C}\right)$ des Methylesters 31 (200 mg, $\left.0.86 \mathrm{mmol}\right)$ in $\mathrm{CH}_{2} \mathrm{Cl}_{2}(5 \mathrm{~mL})$ wird DiBAl-H (1.2 M in Toluol, $2.2 \mathrm{~mL}, 2.67 \mathrm{mmol}$ ) gegeben. Die Lösung wird für eineinhalb Stunden bei dieser Temperatur gerührt, mit MTBE (6.6 mL) verdünnt und auf Raumtemperatur erwärmt. Nach der Zugabe von Wasser $(0.22 \mathrm{~mL})$ wird die Mischung stark gerührt, bis ein weißes Gel entstanden ist. Zu diesem Gel wird $4 \mathrm{M}$ $\mathrm{NaOH}(0.22 \mathrm{~mL})$ und Wasser $(0.44 \mathrm{~mL})$ gegeben. Man lässt die Reaktionsmischung so lange rühren, bis ein weißer Feststoff entstanden ist und trocknet das Reaktionsgemisch dann mit $\mathrm{MgSO}_{4}$. Anschließend wird der Feststoff abfiltriert und das Lösungsmittel im Vakuum entfernt. Nach säulenchromatographischer Reinigung (Hexan/EtOAc $=10: 2)$ erhält man das gewünschte Produkt $32(160 \mathrm{mg}, 0.78 \mathrm{mmol}$, 91\%) als farbloses Öl: [a] ${ }^{20}{ }_{\mathrm{D}}+10.4$ (c 1.00, $\left.\mathrm{CHCl}_{3}\right) ;{ }^{1} \mathrm{H}-\mathrm{NMR}\left(400 \mathrm{MHz}, \mathrm{CDCl}_{3}\right) \delta 3.73$ (dd, $J=9.9,4.4 \mathrm{~Hz}, 1 \mathrm{H}$ ), m (3.68-3.57, 2H), 3.54 (dd, J = 9.9, 7.9 Hz, 1H), 2.83 (br s, $1 \mathrm{H}), 1.99-1.87(\mathrm{~m}, 1 \mathrm{H}), 0.89(\mathrm{~s}, 9 \mathrm{H}), 0.83(\mathrm{~d}, J=6.8 \mathrm{~Hz}, 3 \mathrm{H}), 0.07(\mathrm{~s}, 6 \mathrm{H}) ;{ }^{13} \mathrm{C}-\mathrm{NMR}$ $\left(100 \mathrm{MHz}, \mathrm{CDCl}_{3}\right) \quad \delta 68.75,68.29,37.07,25.86,18.19,13.10,-5.55,-5.60$; HRMS berechnet für $\mathrm{C}_{6} \mathrm{H}_{15} \mathrm{O}_{2} \mathrm{Si}_{1}\left(\mathrm{C}_{10} \mathrm{H}_{24} \mathrm{O}_{2} \mathrm{Si}_{1}\right.$ minus $t$-Butyl): 147.0841, gefunden 147.0842. 


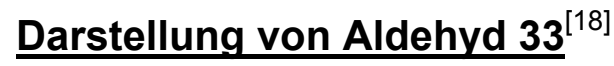

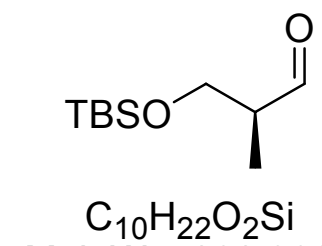

Mol. Wt.: 202,3660

33

$\mathrm{Zu}$ einer Lösung des Alkohols $32(77 \mathrm{mg}, 0.38 \mathrm{mmol})$ in $\mathrm{CH}_{2} \mathrm{Cl}_{2}(2.4 \mathrm{~mL})$ wird bei $0{ }^{\circ} \mathrm{C}$ Dess-Martin Periodinan (191 mg, $0.45 \mathrm{mmol}$ ) gegeben. Die Lösung wird auf Raumtemperatur erwärmt und für eine Stunde gerührt. Die Reaktion wird durch die Zugabe einer Lösung von $\mathrm{Na}_{2} \mathrm{~S}_{2} \mathrm{O}_{3}{ }^{*} 5 \mathrm{H}_{2} \mathrm{O}(0.12 \mathrm{~g})$ in gesättigter, wässriger $\mathrm{NaHCO}_{3}$ Lösung (1.2 mL) gequencht und stark gerührt, bis eine klare Lösung entsteht. Die wässrige Phase wird mit $\mathrm{CH}_{2} \mathrm{Cl}_{2}(2 \times 1.2 \mathrm{~mL})$ extrahiert. Die gesammelten organischen Phasen werden mit $\mathrm{MgSO}_{4}$ getrocknet, der Feststoff wird abfiltriert und das Lösungsmittel im Vakuum entfernt. Nach säulenchromatographischer Reinigung (Hexan/EtOAc $=10: 1)$ erhält man das gewünschte Produkt $33(71 \mathrm{mg}, 0.35$ mmol, 94\%) als farbloses Öl: HRMS berechnet für $\mathrm{C}_{6} \mathrm{H}_{13} \mathrm{O}_{2} \mathrm{Si}_{1} \quad\left(\mathrm{C}_{10} \mathrm{H}_{22} \mathrm{O}_{2} \mathrm{Si}_{1}\right.$-t-Butyl): 145.1389, gefunden 145.1388.

Der Aldehyd 33 wird sofort in die nächste Stufe eingebracht. 


\section{Darstellung von Ethylphosphonsäurebis-(2,2,2-trifluorethyl)ester}

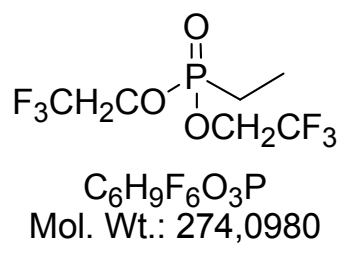

Zu einer Lösung von Trifluorethanol $(7.9 \mathrm{~mL}, 0.11 \mathrm{~mol})$ und Triethylamin $(16.7 \mathrm{~mL}$, $0.12 \mathrm{~mol})$ in THF $(81 \mathrm{~mL})$ wird bei $0{ }^{\circ} \mathrm{C}$ eine Lösung von Ethylphosphonodichlorid (5.8 mL, $0.055 \mathrm{~mol})$ in THF $(11 \mathrm{~mL})$ tropfenweise zugegeben. Danach wird die Reaktionsmischung auf Raumtemperatur erwärmt und für weitere zwei Stunden gerührt. Anschließend wird das $\mathrm{Et}_{3} \mathrm{~N}^{*} \mathrm{HCl}$-Salz abfiltriert und mit THF gewaschen. Das Lösungsmittel wird im Vakuum entfernt. Das zurückbleibende Öl wird destilliert. Nach destillativer Reinigung (20 mbar, Siedetemperatur: $79{ }^{\circ} \mathrm{C}$ ) erhält man das gewünschte Produkt Ethylphosphonsäurebis-(2,2,2-trifluorethyl)ester (13.13 g, $0.048 \mathrm{~mol}, 88 \%)$ als farbloses Öl: ${ }^{1} \mathrm{H}-\mathrm{NMR}\left(400 \mathrm{MHz}, \mathrm{CDCl}_{3}\right) \delta 4.50-4.30(\mathrm{~m}, 4 \mathrm{H})$, 1.95 (dq, $J=18.8,7.7 \mathrm{~Hz}, 2 \mathrm{H}), 1.24(\mathrm{dt}, J=21.6,7.7 \mathrm{~Hz}, 3 \mathrm{H}) ;{ }^{13} \mathrm{C}-\mathrm{NMR}(100 \mathrm{MHz}$, $\left.\mathrm{CDCl}_{3}\right) \delta 122.62(\mathrm{qd}, J=277.5,6.3 \mathrm{~Hz}), 61.90(\mathrm{qd}, J=37.6,6.1 \mathrm{~Hz}), 19.00(\mathrm{~d}$, $J=143.6 \mathrm{~Hz}$ ), $5.93\left(\mathrm{~d}, J=7.1 \mathrm{~Hz}\right.$ ); HRMS berechnet für $\mathrm{C}_{6} \mathrm{H}_{9} \mathrm{O}_{3} \mathrm{P}_{1} \mathrm{~F}_{6}:$ 274.0194, gefunden 274.0193 . 


\section{Darstellung von Phosphonoester 35}

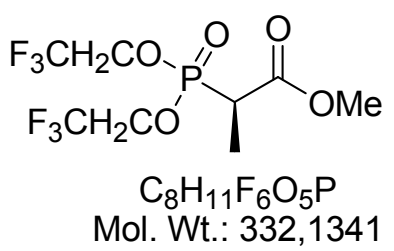

35

Zu einer Lösung von $n$-BuLi (33.5 mL, 2.5 M Lösung in Hexan, $83.69 \mathrm{mmol}$ ) wird bei $-20^{\circ} \mathrm{C}$ eine Lösung von HMDS (19.3 mL, $\left.91.66 \mathrm{~mol}\right)$ in THF (67 mL) gegeben. Nach 20 Minuten wird das Reaktionsgemisch auf $-78{ }^{\circ} \mathrm{C}$ abgekühlt. Dann wird eine Mischung aus Ethylphosphonsäurebis-(2,2,2-trifluorethyl)ester (10.92 g, $39.85 \mathrm{mmol}$ ), Chlorameisensäuremethylester (3.24 mL, $41.85 \mathrm{mmol}$ ) und THF (133 $\mathrm{mL}$ ) hinzugegeben. Anschließend wird die Reaktionsmischung auf $0{ }^{\circ} \mathrm{C}$ erwärmt und durch Zugabe von $1 \mathrm{M}$ wässriger $\mathrm{HCl}(108 \mathrm{~mL}$ ) gequencht (tropfenweise Zugabe und $\mathrm{pH}$-Wert Kontrolle, sobald der pH-Wert $<7$ ist, wird die Zugabe beendet). Dann wird das Reaktionsgemisch auf Raumtemperatur erwärmt. Die Phasen werden getrennt, die wässrige wird mit $\mathrm{CH}_{2} \mathrm{Cl}_{2}$ extrahiert und die gesammelten organischen Phasen

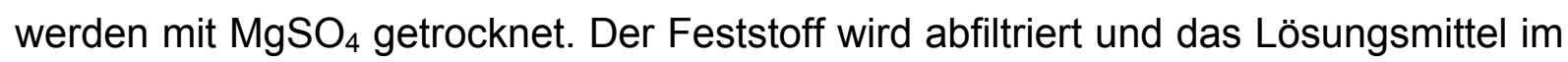
Vakuum entfernt. Das zurückbleibende Öl wird destilliert. (20 mbar, Siedetemperatur: $\left.79{ }^{\circ} \mathrm{C}\right)$. Man erhält das gewünschte Produkt $35(13.13 \mathrm{~g}, 28.30 \mathrm{mmol}, 71 \%)$ als farbloses Öl: $[\alpha]^{20}{ }_{D}+47.8$ (c 1.00, $\mathrm{CHCl}_{3}$ ); ${ }^{1} \mathrm{H}-\mathrm{NMR}\left(400 \mathrm{MHz}, \mathrm{CDCl}_{3}\right) \delta$ 4.55-4.35 (m, $4 \mathrm{H}), 3.80(\mathrm{~s}, 3 \mathrm{H}), 3.22(\mathrm{dq}, J=22.7,7.4 \mathrm{~Hz}, 1 \mathrm{H}), 1.53(\mathrm{dd}, J=19.3,7.4 \mathrm{~Hz}, 3 \mathrm{H})$; ${ }^{13} \mathrm{C}-\mathrm{NMR}\left(100 \mathrm{MHz}, \mathrm{CDCl}_{3}\right) \delta 168.92(\mathrm{~d}, J=3.1 \mathrm{~Hz}), 122.46$ (qd, $J=276.7,7.8 \mathrm{~Hz}$ ), 122.44 (qd, $J=276.7,7.8 \mathrm{~Hz}$ ), 62.60 (qd, $J=37.7,6.0 \mathrm{~Hz}$ ), 62.58 (qd, $J=37.7$, $6.0 \mathrm{~Hz}$ ), 52.98, 39.33 (d, $J=39.3 \mathrm{~Hz}), 11.57$ (d, $J=6.3 \mathrm{~Hz}$ ); HRMS berechnet für $\mathrm{C}_{8} \mathrm{H}_{11} \mathrm{O}_{5} \mathrm{P}_{1} \mathrm{~F}_{6}$ : 332.0248, gefunden 332.0246. 


\section{Darstellung von Methylester 34}

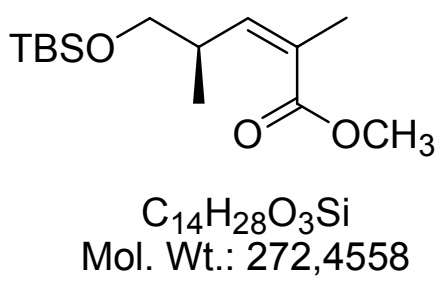

34

Zu einer Lösung von 18-Krone-6 (136 mg, $0.51 \mathrm{mmol})$ in THF $(1 \mathrm{~mL})$ wird bei $-40{ }^{\circ} \mathrm{C}$ tropfenweise der Phosphonoester $35(111 \mathrm{mg}, 0.33 \mathrm{mmol})$ in THF $(0.3 \mathrm{~mL})$ gegeben. Dann wird die Reaktionsmischung auf $-78{ }^{\circ} \mathrm{C}$ abgekühlt und tropfenweise mit KHMDS (0.5 M in Toluol, $0.62 \mathrm{~mL}, 0.31 \mathrm{mmol}$ ) versetzt. Nach 15 Minuten wird der Aldehyd 33 (52 mg, 0.2576 mmol) gelöst in THF (1 mL) zugegeben. Nach eineinhalb Stunden ist die Reaktion beendet und wird durch Zugabe von gesättigter, wässriger $\mathrm{NaHCO}_{3}$-Lösung (1.2 mL) gequencht und auf Raumtemperatur erwärmt. Die Phasen werden getrennt, die wässrige wird mit MTBE extrahiert $(5 \times 20 \mathrm{~mL})$. Die gesammelten organischen Phasen werden mit $\mathrm{MgSO}_{4}$ getrocknet. Der Feststoff wird abfiltriert und das Lösungsmittel im Vakuum entfernt. Nach säulenchromatographischer Reinigung (Hexan/EtOAc = 10:1) erhält man das gewünschte Produkt 34 (69.5 mg, $0.22 \mathrm{mmol}, 85 \%$ ) als farbloses Öl: $[\alpha]^{20} \mathrm{D}-29.8$ (c $1.00, \mathrm{CHCl}_{3}$ ); ${ }^{1} \mathrm{H}-\mathrm{NMR}$ $\left(400 \mathrm{MHz}, \mathrm{CDCl}_{3}\right) \delta 5.75(\mathrm{dd}, J=9.7,1.5 \mathrm{~Hz}, 1 \mathrm{H}), 3.72(\mathrm{~s}, 3 \mathrm{H}), 3.50$ (dd, $J=9.7,6.0$ $\mathrm{Hz}, 1 \mathrm{H}), 3.45(\mathrm{dd}, J=9.7,5.9 \mathrm{~Hz}, 1 \mathrm{H}), 3.33-3.22(\mathrm{~m}, 1 \mathrm{H}), 1.90(\mathrm{~d}, J=1.37 \mathrm{~Hz}, 3 \mathrm{H})$, $0.99(\mathrm{~d}, J=6.49 \mathrm{~Hz}, 3 \mathrm{H}), 0.88(\mathrm{~s}, 9 \mathrm{H}), 0.026(\mathrm{~s}, 3 \mathrm{H}), 0.021(\mathrm{~s}, 3 \mathrm{H}) ;{ }^{13} \mathrm{C}-\mathrm{NMR}$ $\left(100 \mathrm{MHz}_{\mathrm{CDCl}}\right) \quad \delta 168.46,145.53,126.88,67.57,51.24,36.29,25.88,20.84$, 18.30, 16.82, -5.37, -5.41; HRMS berechnet für $\mathrm{C}_{10} \mathrm{H}_{19} \mathrm{O}_{3} \mathrm{Si}_{1}\left(\mathrm{C}_{14} \mathrm{H}_{28} \mathrm{O}_{3} \mathrm{Si}_{1}\right.$ minus t-Butyl): 215.1103, gefunden 215.1103. 


\section{Darstellung von Methylester 22 ${ }^{[15]}$}

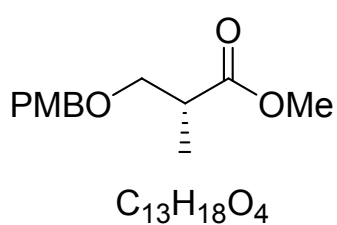

Mol. Wt.: 238,2796

22

Zu einer Mischung von D-(-) $\beta$-Hydroxyisobuttersäuremethylester (21) (7 g, 59.32 mmol) und (4-Methoxybenzyl)-trichloracetimidat $(25,13 \mathrm{~g}, 88.90 \mathrm{mmol})$ in $\mathrm{CH}_{2} \mathrm{Cl}_{2}$ $(170 \mathrm{~mL})$ wird Camphersulfonsäure $(1.38 \mathrm{~g}, 5.90 \mathrm{mmol})$ gegeben und 16 Stunden lang gerührt. Danach saugt man den entstandenen Niederschlag ab, wäscht mit $\mathrm{NaHCO}_{3}$-Lösung und extrahiert mit MTBE. Die gesammelten organischen Phasen werden mit $\mathrm{MgSO}_{4}$ getrocknet. Der Feststoff wird abfiltriert und das Lösungsmittel im Vakuum entfernt. Danach wird etwas Hexan zugegeben, sodass sich erneut ein Niederschlag bildet. Dieser wird abermals abgesaugt, das Lösungsmittel wird wieder am Rotationsverdampfer entfernt und nach säulenchromatographischer Reinigung $($ Hexan/EtOAc $=8: 1)$ erhält man das gewünschte Produkt $22(13.7 \mathrm{~g}, 35.43 \mathrm{mmol}$, 97\%) als farbloses Öl: ${ }^{1} \mathrm{H}-\mathrm{NMR}\left(400 \mathrm{MHz}, \mathrm{CDCl}_{3}\right) \delta 7.26(\mathrm{~d}, \mathrm{~J}=8.6 \mathrm{~Hz}, 2 \mathrm{H}), 6.90$ (d, $J=8.5 \mathrm{~Hz}, 2 \mathrm{H}$ ), 4.47 (s, 2H), $3.82(\mathrm{~s}, 3 \mathrm{H}), 3.71(\mathrm{~s}, 3 \mathrm{H}), 3.65$ (dd, $J=9.2,7.3 \mathrm{~Hz}, 1 \mathrm{H})$, $3.48(\mathrm{dd}, J=9.2,5.9 \mathrm{~Hz}, 1 \mathrm{H}), 2.85-2.74(\mathrm{~m}, 1 \mathrm{H}), 1.19(\mathrm{~d}, J=7.0 \mathrm{~Hz}, 3 \mathrm{H}) ;{ }^{13} \mathrm{C}-\mathrm{NMR}$ $\left(100 \mathrm{MHz}_{\mathrm{CDCl}}\right) \quad \delta 177.42,161.23,132.29,131.26,115.81,74.81,73.71,57.51$, 53.83, 42.36, 16.18; HRMS berechnet für $\mathrm{C}_{13} \mathrm{H}_{18} \mathrm{O}_{4}: 238.1205$, gefunden 238.1204. 


\title{
Darstellung von Alkohol 23 $^{[15]}$
}

\author{
$\mathrm{PMBO} \widehat{\overbrace{\equiv}} \mathrm{OH}$ \\ $\mathrm{C}_{12} \mathrm{H}_{18} \mathrm{O}_{3}$ \\ Mol. Wt.: 210,2695
}

23

Zum Ester 22 (9 g, $35.43 \mathrm{mmol})$ gelöst in $\mathrm{CH}_{2} \mathrm{Cl}_{2}(100 \mathrm{~mL})$ wird bei $-78{ }^{\circ} \mathrm{C}$ tropfenweise DiBAl-H (100 mL, 1M Lösung in Hexan, 100 mmol) gegeben. Anschließend wird für eineinhalb Stunden bei $-78{ }^{\circ} \mathrm{C}$ gerührt. Dann wird das Reaktionsgemisch mit MTBE (100 mL) versetzt und auf Raumtemperatur erwärmt. Nach einer Stunde Rühren wird langsam Wasser $(10 \mathrm{~mL})$ zugetropft. Dabei entsteht nach ca. fünf Minuten ein weißes Gel, welches durch Auflockern mit einem Spatel wieder zum Rühren gebracht werden kann. Man addiert eine Mischung von $2 \mathrm{~N}$ Natronlauge $(6,7 \mathrm{~mL})$ und Wasser $(3,3 \mathrm{~mL})$ und lässt das Reaktionsgemisch rühren, bis ein weißer Feststoff entsteht. Nach Zugabe von $\mathrm{MgSO}_{4}$ wird der gesamte Feststoff abfiltriert. Anschließend wird das Lösungsmittel am Rotationsverdampfer entfernt. Nach säulenchromatographischer Reinigung (Hexan/EtOAc = 8:1) erhält man das gewünschte Produkt $23(13.7 \mathrm{~g}, 35.43 \mathrm{mmol}, 97 \%)$ als farbloses Öl: ${ }^{1} \mathrm{H}-\mathrm{NMR}\left(400 \mathrm{MHz}, \mathrm{CDCl}_{3}\right) \delta 7.27(\mathrm{~d}, J=8.7 \mathrm{~Hz}, 2 \mathrm{H}), 6.90(\mathrm{~d}, J=8.7 \mathrm{~Hz}, 2 \mathrm{H}), 4.47$ $(\mathrm{s}, 2 \mathrm{H}), 3.82(\mathrm{~s}, 3 \mathrm{H}), 3.66-3.58(\mathrm{~m}, 2 \mathrm{H}), 3.54(\mathrm{dd}, J=9.0,4.6 \mathrm{~Hz}, 1 \mathrm{H}), 3.4$ (dd, $J=9.0,8.2 \mathrm{~Hz}, 1 \mathrm{H}), 2.60(\mathrm{br} s, 1 \mathrm{H}), 2.15-2.00(\mathrm{~m}, 1 \mathrm{H}), 0.89(\mathrm{~d}, J=7.0 \mathrm{~Hz}, 3 \mathrm{H})$; ${ }^{13} \mathrm{C}-\mathrm{NMR}\left(100 \mathrm{MHz}, \mathrm{CDCl}_{3}\right) \delta 159.5,130.3,129.4,114.1,75.5,73.4,68.2,55.6$, 35.7, 13.7; HRMS berechnet für $\mathrm{C}_{12} \mathrm{H}_{18} \mathrm{O}_{3}$ : 210.1256, gefunden 210.1257. 


\section{$\underline{\text { Darstellung von Aldehyd 24 }}^{[15]}$}

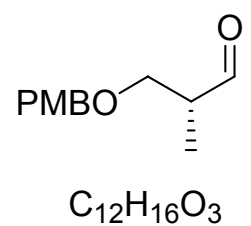

Mol. Wt.: 208,2536

24

Zu einer Lösung des Alkohols $23(7.8 \mathrm{~g}, 37.11 \mathrm{mmol})$ in $\mathrm{CH}_{2} \mathrm{Cl}_{2}(100 \mathrm{~mL})$ wird bei Raumtemperatur Dess-Martin-Periodinan (18.06 g, $42.70 \mathrm{mmol}$ ) gegeben und das Gemisch für eine Stunde gerührt. Dann gibt man $\mathrm{Na}_{2} \mathrm{~S}_{2} \mathrm{O}_{3}{ }^{*} 5 \mathrm{H}_{2} \mathrm{O}(58.9 \mathrm{~g})$ gelöst in gesättigter $\mathrm{NaHCO}_{3}$-Lösung $(117.8 \mathrm{~mL})$ hinzu. Man beobachtet eine leichte Gasentwicklung und lässt ca. 20-30 Minuten rühren, bis die Lösung wieder klar ist. Danach werden die zwei Phasen getrennt, wobei man die wässrige mit $\mathrm{CH}_{2} \mathrm{Cl}_{2}(2 \mathrm{x})$ extrahiert. Die gesammelten organischen Phasen werden mit gesättigter, wässriger $\mathrm{NaCl}$-Lösung gewaschen und anschließend über $\mathrm{MgSO}_{4}$ getrocknet. Der Feststoff wird abfiltriert und das Lösungsmittel am Rotationsverdampfer entfernt. Nach säulenchromatographischer Reinigung (Hexan/EtOAc $=10: 1$ ) erhält man das Produkt 24 (6.6 g, $31.91 \mathrm{mmol}, 86 \%)$ als farbloses Öl: ${ }^{1} \mathrm{H}-\mathrm{NMR}\left(400 \mathrm{MHz}, \mathrm{CDCl}_{3}\right)$ $\delta 9.74(\mathrm{~d}, J=1.70 \mathrm{~Hz}, 1 \mathrm{H}), 7.26(\mathrm{~d}, J=8.7 \mathrm{~Hz}, 2 \mathrm{H}), 6.90(\mathrm{~d}, J=8.7 \mathrm{~Hz}, 2 \mathrm{H}), 4.48$ (s, $2 \mathrm{H}), 3.83(\mathrm{~s}, 3 \mathrm{H}), 3.70-3.60(\mathrm{~m}, 2 \mathrm{H}), 2.73-2.60(\mathrm{~m}, 1 \mathrm{H}), 0.89(\mathrm{~d}, J=7.0 \mathrm{~Hz}, 3 \mathrm{H})$; ${ }^{13} \mathrm{C}-\mathrm{NMR}\left(100 \mathrm{MHz}, \mathrm{CDCl}_{3}\right) \delta 204.02,159.24,129.93,129.20,113.74,72.92,69.68$, 55.12, 46.66, 10.52; HRMS berechnet für $\mathrm{C}_{12} \mathrm{H}_{16} \mathrm{O}_{3}: 208.1099$, gefunden 208.1099. Der Aldehyd 24 wird sofort in die nächste Stufe eingebracht. 


\section{Darstellung von Alken 25}

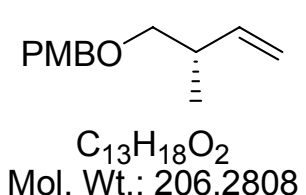

25

Zu einer Suspension von Methyltriphenylphosphoniumbromid (8.6 g, $24.02 \mathrm{mmol}$ ) in THF (206 mL) wird $n$-BuLi $(2.5 \mathrm{M}$ in Hexan, $6.2 \mathrm{~mL}, 15.37 \mathrm{mmol})$ bei $-78^{\circ} \mathrm{C}$ gegeben. Die Reaktionsmischung wird 30 Minuten bei $-78{ }^{\circ} \mathrm{C}$ und 30 Minuten bei Raumtemperatur gerührt. Dann wird sie erneut auf $-78{ }^{\circ} \mathrm{C}$ abgekühlt und der Aldehyd 24 (2 g, $9.61 \mathrm{mmol})$ in THF (27 mL) zugegeben. Es wird fünf Minuten bei $-78{ }^{\circ} \mathrm{C}$ gerührt und anschließend eine Stunde bei Raumtemperatur. Dann wird gesättigte, wässrige $\mathrm{NH}_{4} \mathrm{Cl}$-Lösung zugegeben (200 mL). Die wässrige Phase wird mit MTBE extrahiert. Die gesammelten organischen Phasen werden mit $\mathrm{MgSO}_{4}$ getrocknet. Der Feststoff wird abfiltriert und das Lösungsmittel am Rotationsverdampfer entfernt. Nach säulenchromatographischer Reinigung (Hexan/EtOAc $=20: 1$ ) erhält man das

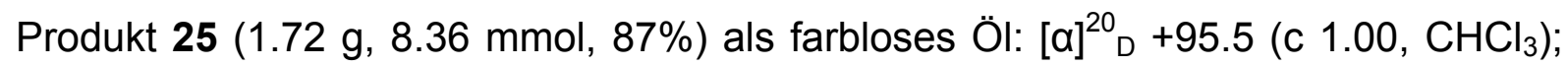
${ }^{1} \mathrm{H}-\mathrm{NMR}\left(400 \mathrm{MHz}, \mathrm{CDCl}_{3}\right) \delta 7.26(\mathrm{~d}, J=8.5 \mathrm{~Hz}, 2 \mathrm{H}), 6.88(\mathrm{~d}, J=8.5 \mathrm{~Hz}, 2 \mathrm{H}), 5.80$ (ddd, $J=17.3,10.3,6.9 \mathrm{~Hz}, 1 \mathrm{H}), 5.10-4.99(\mathrm{~m}, 2 \mathrm{H}), 4.45$ (s, 2H), $3.81(\mathrm{~s}, 3 \mathrm{H}), 3.36$ (dd, $J=9.05,6.66 \mathrm{~Hz}, 1 \mathrm{H}), 3.28(\mathrm{dd}, J=9.05,6.83 \mathrm{~Hz}, 1 \mathrm{H}), 2.55-2.43(\mathrm{~m}, 1 \mathrm{H}), 1.03$ $(\mathrm{d}, J=6.8 \mathrm{~Hz}, 3 \mathrm{H}) ;{ }^{13} \mathrm{C}-\mathrm{NMR}\left(100 \mathrm{MHz}, \mathrm{CDCl}_{3}\right) \delta 159.08,141.36,130.67,129.15$, 113.99, 113.71, 74.74, 72.60, 55.25, 37.79, 16.61; HRMS berechnet für $\mathrm{C}_{13} \mathrm{H}_{18} \mathrm{O}_{2}$ : 206.1307, gefunden 206.1306. 


\section{Darstellung von Allylalkohol 36}

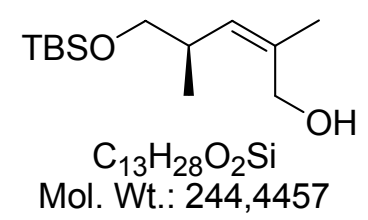

36

$\mathrm{Zu}$ einer Lösung des Esters 34 (253 mg, $0.93 \mathrm{mmol}$ ) in $\mathrm{CH}_{2} \mathrm{Cl}_{2}$ wird bei $-78{ }^{\circ} \mathrm{C}$ DiBAl-H (1.2 M in Toluol, $2.4 \mathrm{~mL}, 2.88 \mathrm{mmol})$ gegeben. Die Lösung wird für eineinhalb Stunden bei dieser Temperatur gerührt, mit MTBE $(7 \mathrm{~mL})$ verdünnt und auf Raumtemperatur erwärmt. Nach der Zugabe von Wasser $(0.24 \mathrm{~mL})$ wird die Reaktionsmischung stark gerührt, bis ein weißes Gel entsteht. Zu diesem Gel wird $4 \mathrm{M} \mathrm{NaOH}(0.24 \mathrm{~mL})$ und Wasser $(0.4 \mathrm{~mL})$ gegeben. Das Reaktionsgemisch wird solange gerührt, bis ein weißer Feststoff entsteht. Dann trocknet man es über $\mathrm{MgSO}_{4}$, filtriert die Feststoffe ab und entfernt das Lösungsmittel im Vakuum. Nach säulenchromatographischer Reinigung (Hexan/EtOAc $=10: 2)$ erhält man das gewünschte Produkt 36 (197.6 mg, $0.81 \mathrm{mmol}, 87 \%$ ) als farbloses Öl: $[\alpha]^{20}{ }_{D}+26.4$ (c 1.00, $\mathrm{CHCl}_{3}$ ); ${ }^{1} \mathrm{H}-\mathrm{NMR}\left(400 \mathrm{MHz}, \mathrm{CDCl}_{3}\right) \delta 4.99$ (d, $\left.J=9.91 \mathrm{~Hz}, 1 \mathrm{H}\right), 4.21$ (dd, $J=$ 11.80, $1.00 \mathrm{~Hz}, 1 \mathrm{H}$ ), $3.81(\mathrm{~d} J=11.80 \mathrm{~Hz}, 1 \mathrm{H}), 3.53(\mathrm{dd} J=9.35,4.89 \mathrm{~Hz}, 1 \mathrm{H}), 3.23$ (t, $J=9.35 \mathrm{~Hz}, 1 \mathrm{H}), 2.78-2.64(\mathrm{~m}, 1 \mathrm{H}), 2.64-2.38(\mathrm{br} \mathrm{s}, 1 \mathrm{H}), 1.80(\mathrm{~d}, J=1.38 \mathrm{~Hz}$, $3 \mathrm{H}), 0.91(\mathrm{~s}, 3 \mathrm{H}), 0.89(\mathrm{~s}, 9 \mathrm{H}), 0.05(\mathrm{~s}, 6 \mathrm{H}) ;{ }^{13} \mathrm{C}-\mathrm{NMR}\left(100 \mathrm{MHz}, \mathrm{CDCl}_{3}\right) \delta 136.64$, $131.72,68.09,62.03,35.04,25.97,22.46,18.53,17.20,-5.45,-5.47$; HRMS berechnet für $\mathrm{C}_{9} \mathrm{H}_{19} \mathrm{O}_{2} \mathrm{Si}_{1}\left(\mathrm{C}_{13} \mathrm{H}_{28} \mathrm{O}_{2} \mathrm{Si}_{1}\right.$ minus $t$-Butyl): 187.1154 , gefunden 187.1153 . 


\section{Darstellung von Allylchlorid 37}

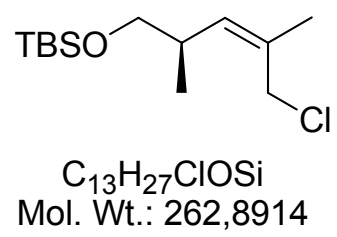

37

Zu einer Lösung des Allylalkohols $36(400 \mathrm{mg}, 1.64 \mathrm{mmol})$ in 2,6-Lutidin $(0.23 \mathrm{~mL}$, $1.96 \mathrm{mmol}$ ) wird bei Raumtemperatur eine Lösung von $\mathrm{LiCl}(77.2 \mathrm{mg}, 1.80 \mathrm{mmol})$ in DMF (1 mL) gegeben. Das Reaktionsgemisch wird auf $0{ }^{\circ} \mathrm{C}$ abgekühlt und dann tropfenweise mit Trifluormethansulfonylchlorid $(0.28 \mathrm{~mL}, 2.68 \mathrm{mmol})$ versetzt. Nach jeweils einer Stunde Rühren bei $0{ }^{\circ} \mathrm{C}$ werden erneut 2,6-Lutidin, $\mathrm{LiCl}$ und Trifluormethansulfonylchlorid zugegeben. Wenn der Allylalkohol 36 vollständig abreagiert ist, wird die Zugabe gestoppt und noch eine weitere Stunde bei $0{ }^{\circ} \mathrm{C}$ gerührt. Anschließend wird die Reaktionsmischung auf Eiswasser ( $8.3 \mathrm{~mL})$ gegeben. Man schüttelt mit MTBE aus, wäscht mit gesättigter, wässriger $\mathrm{CuSO}_{4^{-}}, \mathrm{NaHCO}_{3^{-}}$ und $\mathrm{NaCl}$-Lösung und trocknet die organische Phase über $\mathrm{MgSO}_{4}$. Dann filtriert man den Feststoff ab und entfernt das Lösungsmittel im Vakuum. Nach säulenchromatographischer Reinigung (Hexan/EtOAc $=10: 1)$ erhält man das gewünschte Produkt $37(339.8 \mathrm{mg}, 1.29 \mathrm{mmol}, 79 \%)$ als farbloses Öl: $[\alpha]^{20}-36.6$ (c $\left.1.00, \mathrm{CHCl}_{3}\right) ;{ }^{1} \mathrm{H}-\mathrm{NMR}\left(400 \mathrm{MHz}, \mathrm{CDCl}_{3}\right) \delta 5.15(\mathrm{~d}, J=9.4 \mathrm{~Hz}, 1 \mathrm{H}), 4.18(\mathrm{~d}, J=$ $10.8 \mathrm{~Hz}, 1 \mathrm{H}), 3.99(\mathrm{~d}, J=10.8 \mathrm{~Hz}, 1 \mathrm{H}), 3.4(\mathrm{~d}, J=6.7 \mathrm{~Hz}, 2 \mathrm{H}), 2.67-2.54(\mathrm{~m}, 1 \mathrm{H})$, $1.82(\mathrm{~d}, J=1.4 \mathrm{~Hz}, 3 \mathrm{H}), 0.97(\mathrm{~d}, J=6.7 \mathrm{~Hz}, 3 \mathrm{H}), 0.88(\mathrm{~s}, 9 \mathrm{H}), 0.03(\mathrm{~s}, 3 \mathrm{H}), 0.02(\mathrm{~s}$, $3 \mathrm{H}) ;{ }^{13} \mathrm{C}-\mathrm{NMR}\left(100 \mathrm{MHz}, \mathrm{CDCl}_{3}\right) \delta 134.11,131.71,67.70,44.13,35.55,25.90$, 21.61, 18.32, 17.24, -5.33, -5.41; HRMS berechnet für $\mathrm{C}_{9} \mathrm{H}_{18} \mathrm{O}_{1} \mathrm{Si}_{1} \mathrm{Cl}_{1}\left(\mathrm{C}_{13} \mathrm{H}_{27} \mathrm{O}_{1} \mathrm{Si}_{1} \mathrm{Cl}_{1}\right.$ minus $t$-Butyl): 205.0815, gefunden 205.0815. 


\section{Darstellung von Nitril 38}

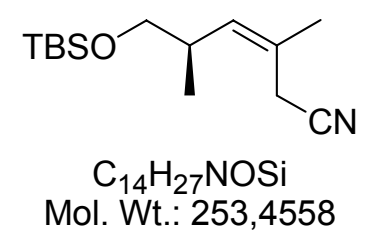

38

$\mathrm{NaCN}$ (76.7 mg, $1.57 \mathrm{mmol}$ ) wird bei $0{ }^{\circ} \mathrm{C}$ zu einer Lösung des Allylchlorids 37 (343 mg, $1.30 \mathrm{mmol})$ in DMF (10.9 mL) gegeben. Nach einer Stunde und 40 Minuten (Zeit muss exakt eingehalten werden, da sich sonst Nebenprodukte bilden!) wird die Reaktionsmischung mit Wasser (44 mL) gequencht. Man extrahiert die wässrige Phase mit MTBE und wäscht die gesammelten organischen Phasen mit gesättigter, wässriger $\mathrm{NaCl}$-Lösung. Man trocknet über $\mathrm{MgSO}_{4}$, filtriert den Feststoff ab und entfernt das Lösungsmittel im Vakuum. Nach säulenchromatographischer Reinigung $($ Hexan/EtOAc = 10:1) erhält man das gewünschte Produkt 38 (198.4 mg, 0.78 mmol,

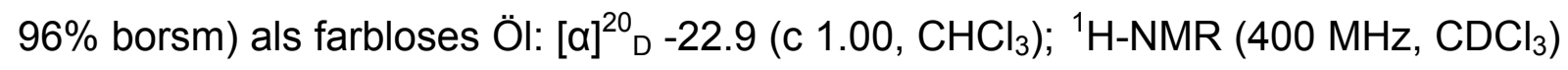
$\delta 5.16(\mathrm{~d}, J=9.9 \mathrm{~Hz}, 1 \mathrm{H}), 3.43(\mathrm{dd}, J=9.8,6.1 \mathrm{~Hz}, 1 \mathrm{H}), 3.36(\mathrm{dd}, J=9.8,7.0 \mathrm{~Hz}$, $1 \mathrm{H}), 3.21(\mathrm{dd}, J=17.4,1.0 \mathrm{~Hz}, 1 \mathrm{H}), 3.02(\mathrm{dd}, J=17.4,0.9 \mathrm{~Hz}, 1 \mathrm{H}), 2.53-2.39$ $(\mathrm{m}, 1 \mathrm{H}), 1.86(\mathrm{~d}, J=1.3 \mathrm{~Hz}, 3 \mathrm{H}), 0.94(\mathrm{~d}, J=6.7 \mathrm{~Hz}, 3 \mathrm{H}), 0.87(\mathrm{~s}, 9 \mathrm{H}), 0.024(\mathrm{~s}, 3 \mathrm{H})$, $0.018(\mathrm{~s}, 3 \mathrm{H}) ;{ }^{13} \mathrm{C}-\mathrm{NMR}\left(100 \mathrm{MHz}, \mathrm{CDCl}_{3}\right) \delta 133.49,124.41,117.86,67.66,35.86$, $25.87,23.02,20.46,18.29,16.87,-5.38,-5.46$; HRMS berechnet für $\mathrm{C}_{10} \mathrm{H}_{18} \mathrm{O}_{1} \mathrm{Si}_{1} \mathrm{~N}_{1}$ $\left(\mathrm{C}_{14} \mathrm{H}_{27} \mathrm{O}_{1} \mathrm{Si}_{1} \mathrm{~N}_{1}\right.$ minus $t$-Butyl): 196.1158, gefunden 196.1157. 


\section{Darstellung von Aldehyd 39}

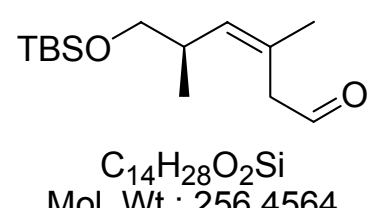

39

DiBAl-H (1.5 M Lösung in Toluol, $0.79 \mathrm{~mL}, 1.18 \mathrm{mmol})$ wird bei $-78{ }^{\circ} \mathrm{C}$ tropfenweise zu einer Lösung des Nitrils 38 (261 mg, $1.03 \mathrm{mmol})$ in $\mathrm{CH}_{2} \mathrm{Cl}_{2}(13.2 \mathrm{~mL})$ gegeben. Nach beendeter Zugabe wird die Reaktionsmischung sofort mit absolutem Ethanol $(0.18 \mathrm{~mL})$ gequencht. Anschließend wird sie auf eine Mischung aus gesättigter, wässriger $\mathrm{NH}_{4} \mathrm{Cl}$-Lösung und EtOAc (je $40 \mathrm{~mL}$ ) gegeben und für eine halbe Stunde gerührt. Dann wird gesättigte, wässrige $\mathrm{Na}_{2} \mathrm{SO}_{4}$-Lösung $(40 \mathrm{~mL})$ hinzugegeben und weitere drei Stunden gerührt. Im Anschluss daran werden die Phasen getrennt und die wässrige wird mit EtOAc extrahiert. Die gesammelten organischen Phasen werden über $\mathrm{MgSO}_{4}$ getrocknet. Dann filtriert man den Feststoff ab und entfernt das Lösungsmittel im Vakuum. Man erhält das gewünschte Produkt 39 (214.0 mg, $834.3 \mu \mathrm{mol}, 81 \%$ ) als farbloses Öl, das ohne Reinigung sofort in die nächste Stufe eingesetzt wird: $[\alpha]^{20}-21.8$ (c 1.00, $\mathrm{CHCl}_{3}$ ); HRMS berechnet für $\mathrm{C}_{10} \mathrm{H}_{19} \mathrm{O}_{2} \mathrm{Si}_{1}$ $\left(\mathrm{C}_{14} \mathrm{H}_{28} \mathrm{O}_{2} \mathrm{Si}_{1}\right.$ minus $t$-Butyl): 199.1859, gefunden 199.1855. 


\section{Darstellung von Dibromolefin 11}

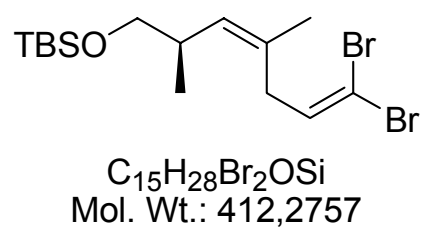

11

Der Aldehyd 39 (2 g, $7.8057 \mathrm{mmol}$ ) wird zu einer Mischung aus Zinkstaub (1 g, 15.61 mmol), Triphenylphosphin $(4.1 \mathrm{~g}, 15.61 \mathrm{mmol})$ und Tetrabromkohlenstoff (5.2 g, $15.5133 \mathrm{mmol})$ in $\mathrm{CH}_{2} \mathrm{Cl}_{2}(60 \mathrm{~mL})$ gegeben, die bei Raumtemperatur für 24-30 Stunden gerührt hat. Anschließend wird für eineinhalb Stunden bei Raumtemperatur gerührt. Dann wird Pentan (227 mL) zu der Reaktionsmischung gegeben und das unlösliche Material wird abfiltriert. Das Lösungsmittel wird im Vakuum entfernt. Nach säulenchromatographischer Reinigung (Hexan/EtOAc $=20: 1$ ) erhält man das gewünschte Produkt 11 (2.9 g, 7.10 mmol, 91\%) als farbloses Öl: [a] ${ }^{20}{ }_{D}-24.7$ (c 1.00 , $\left.\mathrm{CHCl}_{3}\right) ;{ }^{1} \mathrm{H}-\mathrm{NMR}\left(400 \mathrm{MHz}, \mathrm{CDCl}_{3}\right) \delta 6.32(\mathrm{t}, J=7.3 \mathrm{~Hz}, 1 \mathrm{H}), 4.99(\mathrm{~d}, J=8.9 \mathrm{~Hz}$, $1 \mathrm{H}$ ), 3.40 (dd, $J=9.7,6.5 \mathrm{~Hz}, 1 \mathrm{H}), 3.36(\mathrm{dd}, 9.7,6.7 \mathrm{~Hz}, 1 \mathrm{H}), 2.88$ (dd, $J=15.6$, $7.5 \mathrm{~Hz}, 1 \mathrm{H}), 2.8(\mathrm{dd}, J=14.3,7.5 \mathrm{~Hz}, 1 \mathrm{H}), 2.60-2.47(\mathrm{~m}, 1 \mathrm{H}), 1.70(\mathrm{~d}, J=1.38 \mathrm{~Hz}$, $3 \mathrm{H}), 0.92(\mathrm{~d}, J=6.8 \mathrm{~Hz}, 3 \mathrm{H}), 0.89(\mathrm{~s}, 9 \mathrm{H}), 0.04(\mathrm{~s}, 6 \mathrm{H}) ;{ }^{13} \mathrm{C}-\mathrm{NMR}\left(100 \mathrm{MHz}, \mathrm{CDCl}_{3}\right)$ $\delta 136.77,131.20,130.72,89.14,68.07,36.13,35.63,25.95,23.51,18.40,17.50$, -5.30 , -5.32; HRMS berechnet für $\mathrm{C}_{11} \mathrm{H}_{19} \mathrm{O}_{1} \mathrm{Si}_{1} \mathrm{Br}_{2}\left(\mathrm{C}_{15} \mathrm{H}_{28} \mathrm{O}_{1} \mathrm{Si}_{1} \mathrm{Br}_{2}\right.$ minus $t$-Butyl): 353.0276, gefunden 353.0276 . 


\section{Darstellung von Vinylboronsäureester 29}

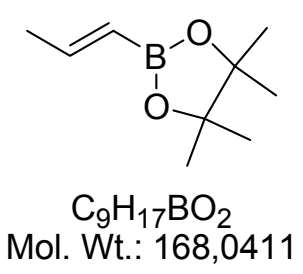

29

Zu einer Lösung von Trimethylborat $(1 \mathrm{~g}, 9.62 \mathrm{mmol})$ in Diethylether $(2.4 \mathrm{~mL})$ wird bei $-78{ }^{\circ} \mathrm{C}$ 1-Propenylmagnesiumbromid $(0.5 \mathrm{M}$ in THF, $24 \mathrm{~mL})$ tropfenweise über eine halbe Stunde hinzugegeben. Man lässt das Reaktionsgemisch eine Stunde bei $-78{ }^{\circ} \mathrm{C}$ rühren und erwärmt dann auf $0{ }^{\circ} \mathrm{C}$. Daraufhin wird $30 \%$ ige $\mathrm{HCl}(16.8 \mathrm{~mL})$ hinzugegeben. Anschließend wird das Reaktionsgemisch eine halbe Stunden bei $0{ }^{\circ} \mathrm{C}$ gerührt und dann auf Raumtemperatur erwärmt. Die organische Phase wird von der wässrigen getrennt. Letztere wird mehrmals mit MTBE extrahiert. Die gesammelten organischen Phasen werden über $\mathrm{MgSO}_{4}$ getrocknet. Dann wird das Lösungsmittel im Vakuum entfernt. Das zurückbleibende Öl wird erneut in Diethylether $(12 \mathrm{~mL})$ gelöst und mit Molsieb $4 \AA$ (1.2 g) und Pinacol $(1.7 \mathrm{~g}$, $14.44 \mathrm{mmol}$ ) versetzt. Das Reaktionsgemisch wird 12 Stunden lang gerührt. Dann filtriert man den Feststoff ab und entfernt das Lösungsmittel im Vakuum. Nach säulenchromatographischer Reinigung (Hexan/EtOAc $=100: 1)$ erhält man das gewünschte Produkt 29 (1.4 g, $8.18 \mathrm{mmol}, 85 \%)$ als farbloses Öl: ${ }^{1} \mathrm{H}-\mathrm{NMR}(400 \mathrm{MHz}$, $\left.\mathrm{CDCl}_{3}\right) \delta$ 6.59-6.46 (m, 1H), $5.35(\mathrm{dq}, J=13.3,1.3 \mathrm{~Hz}, 1 \mathrm{H}), 1.96(\mathrm{dd}, J=6.8,1.7 \mathrm{~Hz}$, $3 \mathrm{H}), 1.27$ (s, 12H); ${ }^{13} \mathrm{C}-\mathrm{NMR}\left(100 \mathrm{MHz}, \mathrm{CDCl}_{3}\right) \quad \delta$ 149.65, 82.78, 24.85, 18.53; HRMS berechnet für $\mathrm{C}_{9} \mathrm{H}_{17} \mathrm{O}_{2} \mathrm{~B}_{1}: 168.0545$, gefunden 168.0545 . 


\section{Darstellung von Vinylboronsäureester 10}

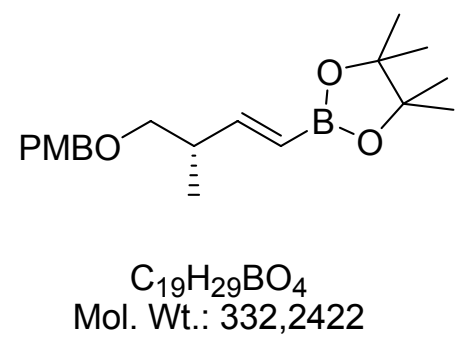

10

Zu einer Lösung von Vinylboronsäureester 29 (1.63 g, 9.70 mmol) und Alken 25 (1 g, $4.85 \mathrm{mmol})$ in $\mathrm{CH}_{2} \mathrm{Cl}_{2}(24 \mathrm{~mL})$ wird Grubbs Katalysator zweiter Generation (0.2 g, $0.24 \mathrm{mmol})$ gegeben und 16 Stunden lang refluxiert. Dann wird das Lösungsmittel entfernt. Nach säulenchromatographischer Reinigung (Hexan/EtOAc $=10: 1)$ erhält man das gewünschte Produkt $10(1.3 \mathrm{~g}, 3.93 \mathrm{mmol}, 81 \%)$ als farbloses Öl: $[\alpha]^{20}$ +78.3 (c 1.00, $\left.\mathrm{CHCl}_{3}\right) ;{ }^{1} \mathrm{H}-\mathrm{NMR}\left(400 \mathrm{MHz}, \mathrm{CDCl}_{3}\right) \delta 7.27$ (d, J=8.6 Hz, 2H), 6.89 (d, $J=8.6 \mathrm{~Hz}, 2 \mathrm{H}), 6.60(\mathrm{dd}, J=18.3,6.49 \mathrm{~Hz}, 1 \mathrm{H}), 5.51(\mathrm{dd}, J=18.3,1.4 \mathrm{~Hz}, 1 \mathrm{H}), 4.46$ (d, $J=1.7 \mathrm{~Hz}, 2 \mathrm{H}$ ), $3.82(\mathrm{~s}, 3 \mathrm{H}), 3.43$ (dd, $J=9.2,6.1 \mathrm{~Hz}, 1 \mathrm{H}), 3.29$ (dd, $J=9.2$, $7.2 \mathrm{~Hz}, 1 \mathrm{H}), 2.64-2.52(\mathrm{~m}, 1 \mathrm{H}), 1.28(\mathrm{~s}, 12 \mathrm{H}), 1.07$ (dd, $J=6.8 \mathrm{~Hz}, 3 \mathrm{H}) ;{ }^{13} \mathrm{C}-\mathrm{NMR}$ $\left(100 \mathrm{MHz}_{\mathrm{CDCl}}\right) \quad \delta 159.13,156.18,130.64,129.23,113.73,83.10,74.15,72.62$, 55.27, 39.45, 24.82, 24.79, 16.29; HRMS berechnet für $\mathrm{C}_{19} \mathrm{H}_{29} \mathrm{O}_{4} \mathrm{~B}_{1}$ : 332.2159, gefunden 332.2158.

Bei dem isolierten Produkt handelt es sich um ein E/Z-Gemisch von 50:1. 


\section{Darstellung von Trien 40}

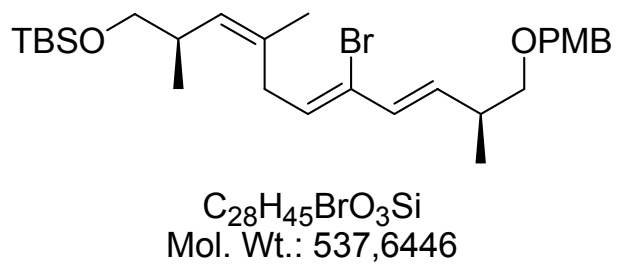

40

Dibromolefin 11 (0.61 g, $1.49 \mathrm{mmol})$ und Vinylboronsäureester 10 (0.99 g, $2.98 \mathrm{mmol})$ werden in einem $\mathrm{THF} / \mathrm{H}_{2} \mathrm{O}$ - Gemisch $(20 \mathrm{~mL} / 4.8 \mathrm{~mL})$ gelöst. Dann entgast man das Reaktionsgemisch, indem man 30 Minuten lang Argon durchströmen lässt. Anschließend gibt man Tetrakis-(triphenylphosphin)-palladium(0) (89.3 mg, $0.077 \mathrm{mmol}$ ) hinzu und fünf Minuten später Thalliumethoxid $(179 \mu \mathrm{L}$, $2.53 \mathrm{mmol}$ ). Nach einer Stunde ist die Reaktion beendet und wird durch die Zugabe von $50 \mathrm{~mL}$ MTBE und $15 \mathrm{~mL} 1 \mathrm{M} \mathrm{NaHSO}_{4}$ gequencht. Das Reaktionsgemisch wird über Celite abfiltriert, dann werden die Phasen getrennt und die wässrige wird mit MTBE extrahiert. Die gesammelten organischen Phasen werden über $\mathrm{MgSO}_{4}$ getrocknet. Der Feststoff wird abfiltriert und das Lösungsmittel im Vakuum entfernt. Nach säulenchromatographischer Reinigung (Hexan/EtOAc $=20: 1$ ) erhält man das gewünschte Produkt $40(0.61 \mathrm{~g}, 1.13 \mathrm{mmol}, 76 \%)$ als farbloses Öl: $[\alpha]^{20}-8.6$ (c $\left.1.00, \mathrm{CHCl}_{3}\right) ;{ }^{1} \mathrm{H}-\mathrm{NMR}\left(400 \mathrm{MHz}, \mathrm{CDCl}_{3}\right) \delta 7.28(\mathrm{~d}, \mathrm{~J}=8.6 \mathrm{~Hz}, 2 \mathrm{H}), 6.90(\mathrm{~d}, \mathrm{~J}=$ $8.6 \mathrm{~Hz}, 2 \mathrm{H}), 6.09$ (d, $J=14.9 \mathrm{~Hz}, 1 \mathrm{H}), 6.01(\mathrm{dd}, J=14.9,6.9 \mathrm{~Hz}, 1 \mathrm{H}), 5.81$ (t, $J=$ $7.1 \mathrm{~Hz}, 1 \mathrm{H}), 5.00(\mathrm{~d}, J=9.4 \mathrm{~Hz}, 1 \mathrm{H}), 4.48(\mathrm{~s}, 2 \mathrm{H}), 3.83(\mathrm{~s}, 3 \mathrm{H}), 3.49-3.29(\mathrm{~m}, 4 \mathrm{H})$, 3.12 (dd, $J=15.3,7.2 \mathrm{~Hz}, 1 \mathrm{H}), 3.03$ (dd, $J=15.3,7.0 \mathrm{~Hz}, 1 \mathrm{H}), 2.68-2.57(\mathrm{~m}, 2 \mathrm{H})$, $1.72(\mathrm{~d}, J=1.3 \mathrm{~Hz}, 3 \mathrm{H}$ ), 1.09 (d, $J=6.8 \mathrm{~Hz}, 3 \mathrm{H}$ ), 0.96 (d, $J=6.7 \mathrm{~Hz}, 3 \mathrm{H}$ ), 0.90 (s, 9H), 0.95 (s, 6H); ${ }^{13} \mathrm{C}-\mathrm{NMR}\left(100 \mathrm{MHz}, \mathrm{CDCl}_{3}\right) \delta 160.42,138.44,134.12,132.16$, 131.88, 131.08, 130.49, 130.16, 127.05, 115.05, 75.87, 73.94, 69.44, 56.56, 38.02, 36.85, 36.04, 27.31, 25.01, 19.71, 18.89, 18.44, -3.92, -3.96; HRMS berechnet für $\mathrm{C}_{24} \mathrm{H}_{36} \mathrm{O}_{3} \mathrm{Si}_{1} \mathrm{Br}_{1}\left(\mathrm{C}_{28} \mathrm{H}_{45} \mathrm{O}_{3} \mathrm{Si}_{1} \mathrm{Br}_{1}\right.$ minus $t$-Butyl): 479.1617, gefunden 479.1610. 


\section{Darstellung von Alkohol 41}

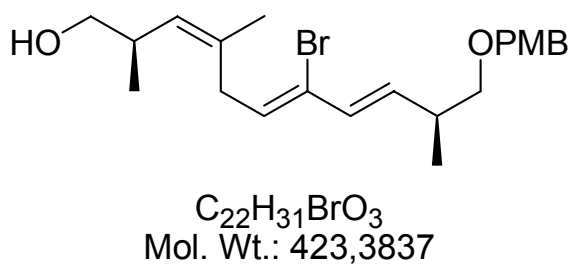

41

Zu einer Lösung des Triens 40 (370 mg, 0.69 mmol) in Pyridin (11.4 mL) und THF (11.4 mL) wird bei Raumtemperatur HF*Pyridin (70\%ig, $7.8 \mathrm{~mL}$ ) zugegeben. Nach fünf Minuten ist die Reaktion beendet und wird durch die Zugabe von gesättigter, wässriger $\mathrm{NaHCO}_{3}$-Lösung $(7.8 \mathrm{~mL})$, EtOAc $(15 \mathrm{~mL})$ und $\mathrm{pH} 7$ Phosphatpuffer $(7.8 \mathrm{~mL})$ gequencht. Die Phasen werden getrennt. Die wässrige Phase wird mit EtOAc ausgeschüttelt. Die gesammelten organischen Phasen trocknet man über $\mathrm{MgSO}_{4}$, filtriert den Feststoff ab und entfernt das Lösungsmittel im Vakuum. Nach säulenchromatographischer Reinigung (Hexan/EtOAc $=2: 1$ ) erhält man das gewünschte Produkt 41 (280.4 mg, $0.66 \mathrm{mmol}, 96 \%)$ als farbloses Öl: $[\alpha]^{20}{ }_{D}+6.4$ (c 1.00, $\left.\mathrm{CHCl}_{3}\right) ;{ }^{1} \mathrm{H}-\mathrm{NMR}\left(400 \mathrm{MHz}, \mathrm{CDCl}_{3}\right) \delta 7.28(\mathrm{~d}, \mathrm{~J}=8.6 \mathrm{~Hz}, 2 \mathrm{H}), 6.90$ (d, J = $8.6 \mathrm{~Hz}, 2 \mathrm{H}), 6.09$ (d, $J=14.8 \mathrm{~Hz}, 1 \mathrm{H}), 6.01$ ( dd, $J=14.8,6.8 \mathrm{~Hz}, 1 \mathrm{H}), 5.82(\mathrm{t}, J=7.3$ $\mathrm{Hz}, 1 \mathrm{H}$ ), 5.00 (d, J = 9.56 Hz, 1H), 4.47 (s, 2H), 3.32 (s, 3H), 3.50 (dd, $J=10.6,5.8$ $\mathrm{Hz}, 1 \mathrm{H}), 3.42-3.29(\mathrm{~m}, 3 \mathrm{H}), 3.14(\mathrm{dd}, J=15.0,7.2 \mathrm{~Hz}, 1 \mathrm{H}), 3.07$ (dd, $J=15.02,7.3$ $\mathrm{Hz}, 1 \mathrm{H}), 2.73-2.62(\mathrm{~m}, 1 \mathrm{H}), 2.61-2.54(\mathrm{~m}, 1 \mathrm{H}), 1.77(\mathrm{~d}, J=1.0 \mathrm{~Hz}, 3 \mathrm{H}), 1.55-1.4$ (br s, 1H), 1.09 (d, $J=6.8 \mathrm{~Hz}, 3 \mathrm{H}), 0.97$ (d, $J=6.8 \mathrm{~Hz}, 3 \mathrm{H}) ;{ }^{13} \mathrm{C}-\mathrm{NMR}(100 \mathrm{MHz}$, $\left.\mathrm{CDCl}_{3}\right) \delta 159.14,137.50,135.05,130.59,130.24,129.22,129.19,128.76,126.04$, $113.77,74.54,72.63,67.88,55.28,36.71,35.66,34.76,23.85,17.27,17.12$; HRMS berechnet für $\mathrm{C}_{22} \mathrm{H}_{31} \mathrm{O}_{3} \mathrm{Br}_{1}$ : 422.1457, gefunden 422.1456 . 


\section{Darstellung von Aldehyd 42}

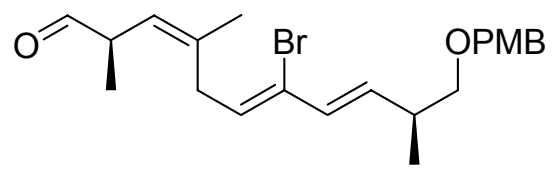

$\mathrm{C}_{22} \mathrm{H}_{29} \mathrm{BrO}_{3}$

Mol. Wt.: 421,3679

42

Zu einer Lösung des Alkohols 41 (86 mg, $203.1 \mu \mathrm{mol}$ ) in $\mathrm{CH}_{2} \mathrm{Cl}_{2}(2.7 \mathrm{~mL}$ ) wird bei Raumtemperatur Dess-Martin Periodinan (103.2 mg, $243.8 \mu \mathrm{mol})$ gegeben und das Gemisch für eine Stunde gerührt. Dann gibt man $\mathrm{Na}_{2} \mathrm{~S}_{2} \mathrm{O}_{3}{ }^{*} 5 \mathrm{H}_{2} \mathrm{O}(143 \mathrm{mg})$ gelöst in gesättigter, wässriger $\mathrm{NaHCO}_{3}$-Lösung $(1.3 \mathrm{~mL})$ hinzu und rührt, bis die Lösung klar ist. Danach werden die Phasen getrennt, wobei man die wässrige mit $\mathrm{CH}_{2} \mathrm{Cl}_{2}(3 \mathrm{x})$ extrahiert. Die gesammelten organischen Phasen werden mit gesättigter, wässriger $\mathrm{NaCl}-\mathrm{Lösung}$ gewaschen und anschließend über $\mathrm{MgSO}_{4}$ getrocknet. Der Feststoff wird abfiltriert und anschließend wird das Lösungsmittel am Rotationsverdampfer entfernt. Nach säulenchromatographischer Reinigung (Hexan/EtOAc $=10: 1)$ erhält man das gewünschte Produkt 42 (74.47 mg, $176.7 \mu \mathrm{mol}, 87 \%)$ als farbloses Öl: $[\alpha]_{D}^{20}+10.9$ (c 1.00, $\mathrm{CHCl}_{3}$ ); HRMS berechnet für $\mathrm{C}_{22} \mathrm{H}_{29} \mathrm{O}_{3} \mathrm{Br}_{1}: 420.1300$, gefunden 420.1305 .

Der Aldehyd $\mathbf{4 2}$ wird sofort in die nächste Stufe eingesetzt. 


\section{Darstellung von (Triphenylphosphoranyliden)essigsäureethylester}

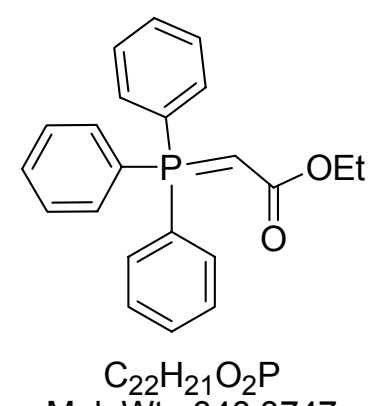

Mol. Wt.: 348,3747

Eine Lösung von Triphenylphosphin $(28.8 \mathrm{~g}, 108 \mathrm{mmol})$ in EtOAc $(200 \mathrm{~mL})$ wird tropfenweise mit einer Lösung von Bromessigsäureethylester $(10.7 \mathrm{~mL}, 17.9 \mathrm{~g}$, $108 \mathrm{mmol})$ in EtOAc $(40 \mathrm{~mL})$ versetzt und bei Raumtemperatur für 19 Stunden gerührt, wobei ein weißer Niederschlag ausfällt. Der Feststoff wird abfiltriert und im Hochvakuum getrocknet. Anschließend wird er in $\mathrm{CH}_{2} \mathrm{Cl}_{2}$ gelöst $(200 \mathrm{~mL})$ und mit $1 \mathrm{M}$ Natronlauge $(160 \mathrm{~mL})$ in einem Scheidetrichter gemischt. Nach dem Trennen der Phasen wird die wässrige Phase noch zweimal mit $\mathrm{CH}_{2} \mathrm{Cl}_{2}$ (je $80 \mathrm{~mL}$ ) extrahiert. Die gesammelten organischen Phasen werden mit gesättigter, wässriger $\mathrm{NaCl}-\mathrm{Lösung}$ gewaschen und über $\mathrm{MgSO}_{4}$ getrocknet. Das Lösungsmittel wird am Rotationsverdampfer entfernt und der zurückbleibende weiße Feststoff im Hochvakuum getrocknet: ${ }^{1} \mathrm{H}-\mathrm{NMR}\left(200 \mathrm{MHz}, \mathrm{CDCl}_{3}\right) \delta 7.73-7.42(\mathrm{~m}, 15 \mathrm{H}), 4.02$ (br s, 2H), $2.92\left(\right.$ br s, 1H), 1.15 (br s, 3H); HRMS berechnet für $\mathrm{C}_{22} \mathrm{H}_{21} \mathrm{O}_{2} \mathrm{P}_{1}$ : 348.1279 , gefunden 348.1278 . 


\section{Darstellung von Ethylester 9}

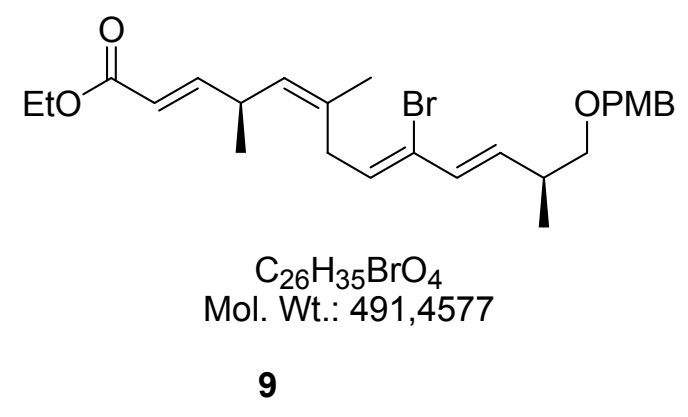

$\mathrm{Zu}$ einer Lösung des Aldehyds 42 (68 mg, $161.4 \mu \mathrm{mol})$ in $\mathrm{CH}_{2} \mathrm{Cl}_{2}$ (3 mL) wird (Triphenylphosphoranyliden)essigsäureethylester (112.36 mg, $322.8 \mu \mathrm{mol})$ gegeben und zwei Stunden lang bei Raumtemperatur gerührt. Dann wird das Lösungsmittel im Vakuum entfernt. Nach säulenchromatographischer Reinigung (Hexan/EtOAc = 10:1) erhält man das gewünschte Produkt 9 (70.59 mg, $143.6 \mu \mathrm{mol}, 89 \%)$ als farbloses Öl: $[\mathrm{\alpha}]^{20}{ }_{\mathrm{D}}-45.0$ (c 1.00, $\mathrm{CHCl}_{3}$ ); ${ }^{1} \mathrm{H}-\mathrm{NMR}\left(400 \mathrm{MHz}, \mathrm{CDCl}_{3}\right) \delta 7.28(\mathrm{~d}, \mathrm{~J}=8.5 \mathrm{~Hz}, 2 \mathrm{H})$, $6.90(\mathrm{dd}, J=14.0,7.9 \mathrm{~Hz}, 1 \mathrm{H}), 6.89(\mathrm{~d}, J=8.5 \mathrm{~Hz}, 2 \mathrm{H}), 6.15-5.97(\mathrm{~m}, 2 \mathrm{H}), 5.84-$ $5.73(\mathrm{~m}, 2 \mathrm{H}), 5.05$ (d, J = 9.2 Hz, 1H), 4.47 (s, 2H), 4.20 (q, J = 7.2 Hz, 2H), 3.82 (s, $3 \mathrm{H}$ ), $3.42-3.26(\mathrm{~m}, 3 \mathrm{H}), 3.09$ (dd, $J=15.4,7.0 \mathrm{~Hz}, 1 \mathrm{H}), 3.01$ (dd, $J=15.4,7.2 \mathrm{~Hz}$, 1H), 2.62 (qui, $J=6.7 \mathrm{~Hz}, 1 \mathrm{H}$ ), $1.75(\mathrm{~s}, 3 \mathrm{H}), 1.31$ (t, $J=7.2 \mathrm{~Hz}, 3 \mathrm{H}), 1.14$ (d, $J=$ $6.1 \mathrm{~Hz}, 3 \mathrm{H}), 1.09$ (d, J = 6.8 Hz, 3H); ${ }^{13} \mathrm{C}-\mathrm{NMR}\left(100 \mathrm{MHz}, \mathrm{CDCl}_{3}\right) \delta 166.96,159.14$, 152. 62 , 137.59, 133.83, 130.57, 129.72, 129.19, 128.70, 128.19, 126.16, 119.26, 113.76, 74.54, 72.64, 60.22, 55.26, 36.69, 35.31, 34.63, 23.70, 20.25, 17.10, 14.30; HRMS berechnet für $\mathrm{C}_{26} \mathrm{H}_{35} \mathrm{O}_{4} \mathrm{Br}_{1}: 490.1719$, gefunden 490.1719. 


\section{Darstellung von Ethylester 65}

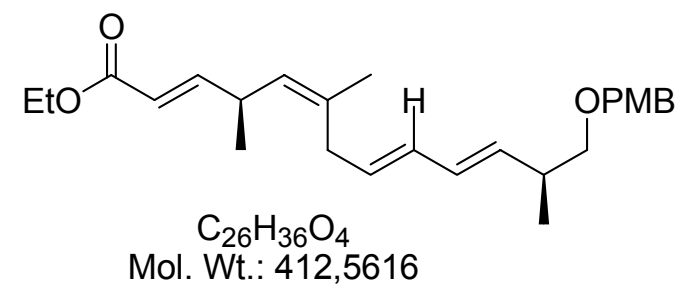

65

Zum Ester 9 (50 mg, $101.7 \mu \mathrm{mol})$ gelöst in $\mathrm{MeOH}(9 \mathrm{~mL}$ ) wird bei Raumtemperatur portionsweise Na/Hg (5 \%) (1.5 g) gegeben. Nach zwei Stunden wird die Reaktion beendet, indem man MTBE (150 mL) zum Reaktionsgemisch gibt. Dann wäscht man die Reaktionslösung mit $1 \mathrm{M} \mathrm{HCl}$, gesättigter, wässriger $\mathrm{Na}_{2} \mathrm{SO}_{4}$-Lösung und Wasser. Anschließend trocknet man die organische Phase mit $\mathrm{MgSO}_{4}$, filtriert den Feststoff $a b$ und entfernt das Lösungsmittel im Vakuum. Nach säulenchromatographischer Reinigung (Hexan/EtOAc $=10: 1$ ) erhält man das gewünschte Produkt $65(25.60 \mathrm{mg}, 62.1 \mu \mathrm{mol}, 61 \%)$ als farbloses Öl: $[\alpha]^{20}-26.7$ (c 1.00, $\left.\mathrm{CHCl}_{3}\right) ;{ }^{1} \mathrm{H}-\mathrm{NMR}\left(400 \mathrm{MHz}, \mathrm{CDCl}_{3}\right) \delta 7.29$ (d, $\left.J=8.5 \mathrm{~Hz}, 2 \mathrm{H}\right), 6.91$ (dd, $J=$ 14.1, 7.8 Hz, 1H), $6.87(\mathrm{~d}, J=8.5 \mathrm{~Hz}, 2 \mathrm{H}), 6.23-5.91(\mathrm{~m}, 3 \mathrm{H}), 5.81-5.71(\mathrm{~m}, 2 \mathrm{H})$, $5.11(\mathrm{~d}, J=9.3 \mathrm{~Hz}, 1 \mathrm{H}), 4.53(\mathrm{~s}, 2 \mathrm{H}), 4.23(\mathrm{q}, J=7.3 \mathrm{~Hz}, 2 \mathrm{H}), 3.84(\mathrm{~s}, 3 \mathrm{H}), 3.49-$ $3.25(\mathrm{~m}, 3 \mathrm{H}), 3.10(\mathrm{dd}, J=15.2,7.1 \mathrm{~Hz}, 1 \mathrm{H}), 3.08(\mathrm{dd}, J=15.2,7.4 \mathrm{~Hz}, 1 \mathrm{H}), 2.65(\mathrm{~m}$, $1 \mathrm{H}), 1.76(\mathrm{~s}, 3 \mathrm{H}), 1.33(\mathrm{t}, J=7.3 \mathrm{~Hz}, 3 \mathrm{H}), 1.11(\mathrm{~d}, J=6.1 \mathrm{~Hz}, 3 \mathrm{H}), 1.08(\mathrm{~d}, J=$ $6.8 \mathrm{~Hz}, 3 \mathrm{H}) ;{ }^{13} \mathrm{C}-\mathrm{NMR}\left(100 \mathrm{MHz}, \mathrm{CDCl}_{3}\right) \delta 166.96,159.11,152.52,137.64,131.81$, $130.80,129.34,129.03,128.73,128.22,126.05,119.34,113.73,74.57,72.34$, $60.11,55.23,36.62,35.14,34.67,23.71,20.33,17.17,14.31$; HRMS berechnet für $\mathrm{C}_{26} \mathrm{H}_{36} \mathrm{O}_{4}:$ 412.2614, gefunden 412.2614 . 


\section{Darstellung von Lacton 8}

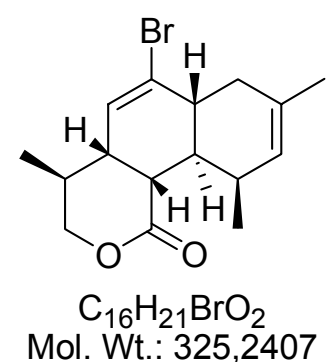

8

Zu dem Ester 9 (100 mg, $203.48 \mathrm{mmol}$ ) und Toluol (50 mL) in einem geschlossenen Gefäß ohne Druckausgleich wird bei Raumtemperatur $\mathrm{BF}_{3}{ }^{*} \mathrm{OEt}_{2}(0.05 \mathrm{~mL})$ gegeben. Nach fünf Minuten ist die PMB-Entschützung komplett und man erhitzt für drei Stunden auf $85{ }^{\circ} \mathrm{C}$. Nachdem das Reaktionsgemisch wieder auf Raumtemperatur abgekühlt ist, wäscht man es mit gesättigter, wässriger $\mathrm{NaHCO}_{3}$-Lösung und extrahiert die wässrige Phase mit MTBE. Die gesammelten organischen Phasen werden mit $\mathrm{MgSO}_{4}$ getrocknet. Der Feststoff wird abfiltriert und anschließend wird das Lösungsmittel am Rotationsverdampfer entfernt. Nach säulenchromatographischer Reinigung (Hexan/EtOAc $=10: 1$ ) erhält man das gewünschte Produkt 8 (38.38 mg, $118.02 \mathrm{mmol}, 58 \%)$ als farblosen Feststoff: $[\alpha]^{20}$ +235.9 (c 1.00, $\left.\mathrm{CHCl}_{3}\right) ;{ }^{1} \mathrm{H}-\mathrm{NMR}\left(400 \mathrm{MHz} \mathrm{CDCl}_{3}\right) \delta 6.21(\mathrm{dd}, J=5.3,2.2 \mathrm{~Hz}, 1 \mathrm{H})$, 5.20 (s, 1H), 4.42 (dd, J = 11.3, $5.3 \mathrm{~Hz}, 1 \mathrm{H}$ ), 3.92 (dd, J = 11.3, $9.2 \mathrm{~Hz}, 1 \mathrm{H}), 2.65$ (dd, $J=10.2,5.5 \mathrm{~Hz}, 1 \mathrm{H}), 2.50-2.34(\mathrm{~m}, 3 \mathrm{H}), 2.31-2.22(\mathrm{~m}, 1 \mathrm{H}), 2.18-2.04(\mathrm{~m}, 1 \mathrm{H})$, $1.81(\mathrm{t}, J=13.7 \mathrm{~Hz}, 1 \mathrm{H}), 1.72(\mathrm{~s}, 3 \mathrm{H}), 1.66(\mathrm{q}, J=10.01 \mathrm{~Hz}, 1 \mathrm{H}), 1.09(\mathrm{~d}, J=6.5 \mathrm{~Hz}$, $3 \mathrm{H}), 0.94(\mathrm{~d}, J=6.5 \mathrm{~Hz}, 3 \mathrm{H}) ;{ }^{13} \mathrm{C}-\mathrm{NMR}\left(100 \mathrm{MHz}, \mathrm{CDCl}_{3}\right) \delta 173.63,131.17,130.21$, 129.31, 127.81, 73.60, 45.26, 44.71, 44.38, 42.71, 36.41, 34.37, 31.73, 23.37, 21.65, 15.57; HRMS berechnet für $\mathrm{C}_{16} \mathrm{H}_{21} \mathrm{O}_{2} \mathrm{Br}_{1}$ : 324.0725, gefunden 324.0722.

Als Nebenprodukt entsteht bei der Reaktion das Lacton 46 (2.95 mg, 9.08 mmol, $4 \%$ ). Das Diastereomerenverhältnis bei dieser Reaktion beträgt 8:46 = 13:1. 


\section{Darstellung von Lacton 46}

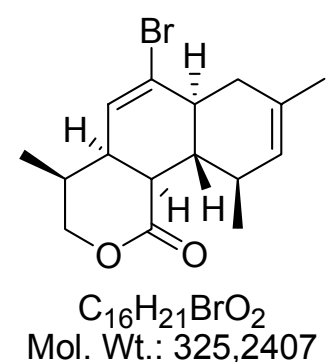

46

Zu einer Lösung des Esters 9 (100 mg, $203.48 \mathrm{mmol})$ in Toluol $(50 \mathrm{~mL})$ wird bei Raumtemperatur $\mathrm{BF}_{3}{ }^{*} \mathrm{OEt}_{2}(0.05 \mathrm{~mL})$ gegeben. Nach fünf Minuten ist die PMBEntschützung komplett und man rührt für 24 Stunden bei $-40{ }^{\circ} \mathrm{C}$. Nachdem das Reaktionsgemisch wieder auf Raumtemperatur erwärmt ist, wäscht man es mit gesättigter, wässriger $\mathrm{NaHCO}_{3}$-Lösung und extrahiert die wässrige Phase mit MTBE. Die gesammelten organischen Phasen werden mit $\mathrm{MgSO}_{4}$ getrocknet. Der Feststoff wird abfiltriert und anschließend wird das Lösungsmittel am Rotationsverdampfer entfernt. Nach säulenchromatographischer Reinigung (Hexan/EtOAc $=10: 1)$ erhält man das gewünschte Produkt $46(8.60 \mathrm{mg}, 26.45 \mathrm{mmol}, 13 \%)$ als farblosen Feststoff: $[\alpha]^{20}{ }_{D}-263.3$ (c 1.00, $\mathrm{CHCl}_{3}$ ); ${ }^{1} \mathrm{H}-\mathrm{NMR}\left(400 \mathrm{MHz}, \mathrm{CDCl}_{3}\right) \delta 6.17$ (dd, $\mathrm{J}=$ 6.0, $1.9 \mathrm{~Hz}, 1 \mathrm{H}), 5.44(\mathrm{~d}, J=5.1 \mathrm{~Hz}, 1 \mathrm{H}), 4.28(\mathrm{dd}, J=11.3,5.1 \mathrm{~Hz}, 1 \mathrm{H}), 3.8(\mathrm{t}, J=$ $11.1 \mathrm{~Hz}, 1 \mathrm{H}), 2.76(\mathrm{dd}, J=12.6,5.5 \mathrm{~Hz}, 1 \mathrm{H}), 2.61-2.43(\mathrm{~m}, 2 \mathrm{H}), 2.34-2.21(\mathrm{~m}, 2 \mathrm{H})$, 2.16-2.01 (m, 2H), $1.77(\mathrm{dd}, J=16.4,11.9 \mathrm{~Hz}, 1 \mathrm{H}), 1.67(\mathrm{~s}, 3 \mathrm{H}), 1.10(\mathrm{~d}, J=6.5 \mathrm{~Hz}$, $3 \mathrm{H}), 1.00(\mathrm{~d}, J=6.8 \mathrm{~Hz}, 3 \mathrm{H}) ;{ }^{13} \mathrm{C}-\mathrm{NMR}\left(100 \mathrm{MHz}, \mathrm{CDCl}_{3}\right) \delta 171.47,131.45,130.21$, 128.36, 128.32, 74.43, 42.11, 40.80, 40.77, 38.62, 38.26, 32.79, 31.81, 23.04, 15.31, 14.34; HRMS berechnet für $\mathrm{C}_{16} \mathrm{H}_{21} \mathrm{O}_{2} \mathrm{Br}_{1}$ : 324.0725 , gefunden 324.0723.

Als Nebenprodukt entsteht bei der Reaktion das Lacton 8. Das Diastereomerenverhältnis bei dieser Reaktion beträgt 46:8 = 4:1. 


\section{Darstellung von Lacton 66}

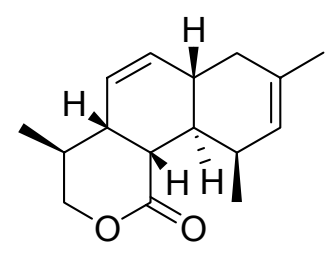

$\mathrm{C}_{16} \mathrm{H}_{22} \mathrm{O}_{2}$

Mol. Wt.: 246,3447

66

Zum Lacton 8 (100 mg, $0.31 \mathrm{mmol})$ gelöst in $\mathrm{MeOH} \mathrm{(18} \mathrm{mL)} \mathrm{wird} \mathrm{bei}$ Raumtemperatur portionsweise $\mathrm{Na} / \mathrm{Hg}$ (5\%) (3.0 g) gegeben. Nach zwei Stunden wird die Reaktion beendet, indem man MTBE (270 mL) zum Reaktionsgemisch gibt. Dann wäscht man die Reaktionslösung mit $1 \mathrm{M} \mathrm{HCl}$, gesättigter, wässriger $\mathrm{Na}_{2} \mathrm{SO}_{4}$ Lösung und Wasser. Anschließend trocknet man die organische Phase mit $\mathrm{MgSO}_{4}$, filtriert den Feststoff ab und entfernt das Lösungsmittel im Vakuum. Nach säulenchromatographischer Reinigung (Hexan/EtOAc $=10: 1$ ) erhält man das gewünschte Produkt $66(70.26 \mathrm{mg}, 0.29 \mathrm{mmol}, 92 \%)$ als farblosen Feststoff: $[\alpha]^{20}{ }_{D}$ +187.3 (c 1.00, $\mathrm{CHCl}_{3}$ ); ${ }^{1} \mathrm{H}-\mathrm{NMR}\left(400 \mathrm{MHz} \mathrm{CDCl}_{3}\right) \delta 5.74$ (ddd, $J=9.9,4.1,2.4 \mathrm{~Hz}$, $1 \mathrm{H}), 5.67(\mathrm{~d}, J=9.9 \mathrm{~Hz}, 1 \mathrm{H}), 5.20(\mathrm{~s}, 1 \mathrm{H}), 4.41(\mathrm{dd}, J=11.3,5.1 \mathrm{~Hz}, 1 \mathrm{H}), 3.95$ (dd, $J=11.3,9.2 \mathrm{~Hz}, 1 \mathrm{H}), 2.61(\mathrm{dd}, J=9.9,6.1 \mathrm{~Hz}, 1 \mathrm{H}), 2.41-2.31(\mathrm{~m}, 1 \mathrm{H}), 2.28-2.21$ (m, 1H), $2.17-1.99(\mathrm{~m}, 2 \mathrm{H}), 1.99$ (dd, $J=16.6,4.3 \mathrm{~Hz}, 1 \mathrm{H}), 1.79(\mathrm{t}, J=14.3 \mathrm{~Hz}$, 1H), $1.70(\mathrm{~s}, 3 \mathrm{H}), 1.43$ (q, J = $9.9 \mathrm{~Hz}, 1 \mathrm{H}), 1.09$ (d, J = 6.8 Hz, 3H), 0.94 (d, J = $6.8 \mathrm{~Hz}, 3 \mathrm{H}) ;{ }^{13} \mathrm{C}-\mathrm{NMR}\left(100 \mathrm{MHz}, \mathrm{CDCl}_{3}\right) \delta 174.78,133.75,131.64,128.20,126.89$, 73.34, 45.64, 42.47, 40.57, 38.57, 36.43, 34.40, 32.90, 23.37, 21.39, 15.94; HRMS berechnet für $\mathrm{C}_{16} \mathrm{H}_{22} \mathrm{O}_{2}: 246.1620$, gefunden 246.1619. 


\section{Darstellung von Lacton 67}

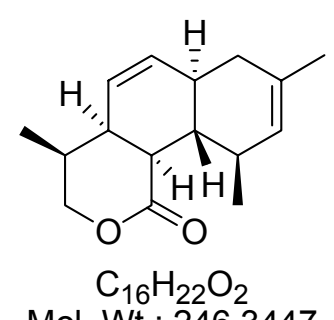

67

Zum Lacton 46 (100 mg, $0.31 \mathrm{mmol}$ ) gelöst in $\mathrm{MeOH}(18 \mathrm{~mL})$ wird bei Raumtemperatur portionsweise $\mathrm{Na} / \mathrm{Hg}(5 \%)(3.0 \mathrm{~g})$ gegeben. Nach zwei Stunden wird die Reaktion beendet, indem man MTBE $(270 \mathrm{~mL})$ zum Reaktionsgemisch gibt. Dann wäscht man die Reaktionslösung mit $1 \mathrm{M} \mathrm{HCl}$, gesättigter, wässriger $\mathrm{Na}_{2} \mathrm{SO}_{4}$ Lösung und Wasser. Anschließend trocknet man die organische Phase mit $\mathrm{MgSO}_{4}$, filtriert den Feststoff ab und entfernt das Lösungsmittel im Vakuum. Nach säulenchromatographischer Reinigung (Hexan/EtOAc $=10: 1)$ erhält man das gewünschte Produkt $67(70.26 \mathrm{mg}, 0.29 \mathrm{mmol}, 92 \%)$ als farblosen Feststoff: $[\alpha]^{20} \mathrm{D}$ -312.9 (c 1.00, $\mathrm{CHCl}_{3}$ ); ${ }^{1} \mathrm{H}-\mathrm{NMR}\left(400 \mathrm{MHz}, \mathrm{CDCl}_{3}\right) \delta 5.76$ (ddd, $J=9.9,4.9,2.1 \mathrm{~Hz}$, $1 \mathrm{H}), 5.70(\mathrm{~d}, J=9.9 \mathrm{~Hz}, 1 \mathrm{H}), 5.48(\mathrm{~d}, J=5.5 \mathrm{~Hz}, 1 \mathrm{H}), 4.33(\mathrm{dd}, J=11.2,4.8 \mathrm{~Hz}, 1 \mathrm{H})$, 3.87 (dd, $J=11.2,10.5 \mathrm{~Hz}, 1 \mathrm{H}), 2.76(\mathrm{dd}, J=12.3,5.8 \mathrm{~Hz}, 1 \mathrm{H}), 2.41-2.31(\mathrm{~m}, 1 \mathrm{H})$, 2.30-2.20 (m, 2H), $2.09(\mathrm{dd}, J=17.1,5.5 \mathrm{~Hz}, 1 \mathrm{H}), 2.05-1.96(\mathrm{~m}, 1 \mathrm{H}), 1.92-1.83(\mathrm{~m}$, $1 \mathrm{H}), 1.74(\mathrm{dd}, J=16.6,12.8 \mathrm{~Hz}, 1 \mathrm{H}), 1.67(\mathrm{~s}, 3 \mathrm{H}), 1.13(\mathrm{~d}, J=6.8 \mathrm{~Hz}, 3 \mathrm{H}), 1.03(\mathrm{~d}$, $J=6.8 \mathrm{~Hz}, 3 \mathrm{H}) ;{ }^{13} \mathrm{C}-\mathrm{NMR}\left(100 \mathrm{MHz}, \mathrm{CDCl}_{3}\right) \delta 172.54,132.44,131.49,128.79$, 125.43, 74.30, 41.36, 39.82, 38.51, 38.36, 32.63, 32.19, 31.17, 23.13, 15.47, 14.73; HRMS berechnet für $\mathrm{C}_{16} \mathrm{H}_{22} \mathrm{O}_{2}: 246.1620$, gefunden 246.1620 . 


\section{Darstellung von Säure 69}

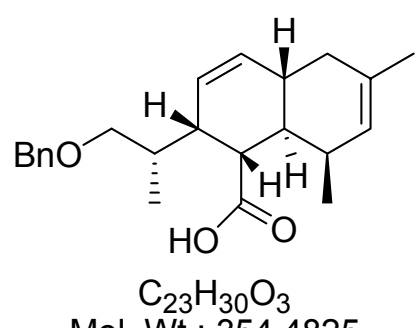

Mol. Wt.: 354,4825

69

Das Lacton 66 (55 mg, $0.22 \mathrm{mmol}$ ), gelöst in Toluol $(0.5 \mathrm{~mL})$, wird mit KOH (63 mg, $1.12 \mathrm{mmol}$ ) und Benzylchlorid $(90.9 \mathrm{mg}, 0.72 \mathrm{mmol}$ ) versetzt und 16 Stunden lang refluxiert. Dann wird das Reaktionsgemisch mit Wasser $(1 \mathrm{~mL})$ und MTBE $(2 \mathrm{~mL})$ gequencht. Die organische Phase wird abgetrennt und die wässrige wird so lange mit $\mathrm{HCl}(2 \mathrm{M})$ versetzt, bis sie einen $\mathrm{pH}-$ Wert von drei hat. Anschließend wird die wässrige Phase mit MTBE extrahiert. Die gesammelten Extrakte werden mit gesättigter, wässriger $\mathrm{NaCl}$-Lösung gewaschen. Die organische Phasen trocknet man mit $\mathrm{MgSO}_{4}$, filtriert den Feststoff ab und entfernt das Lösungsmittel im Vakuum. Die entstandene Säure 69 wird ohne chromatographische Reinigung in die nächste Stufe eingesetzt. 


\section{Darstellung von Alkohol 70}

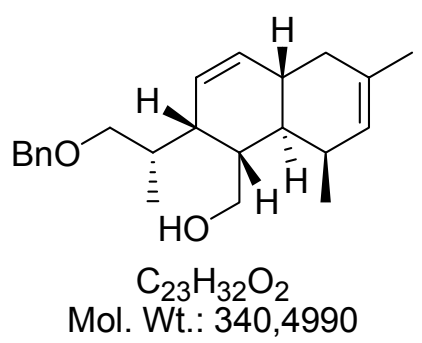

70

Die Säure 69 (80 mg, $0.22 \mathrm{mmol})$, gelöst in Toluol $(2.5 \mathrm{~mL})$, wird bei $-78{ }^{\circ} \mathrm{C}$ mit DiBAl-H versetzt. Dann lässt man das Reaktionsgemisch auf Raumtemperatur erwärmen und rührt weitere eineinhalb Stunden. Anschließend gibt man MTBE (3 mL), Wasser $(0.17 \mathrm{~mL}), \mathrm{NaOH}(4 \mathrm{M}, 0.34 \mathrm{~mL})$ und noch einmal Wasser $(0.17 \mathrm{~mL})$ hinzu. Man lässt so lange rühren, bis ein weißer Feststoff entsteht. Dann trocknet man mit $\mathrm{MgSO}_{4}$, filtriert den Feststoff ab und entfernt das Lösungsmittel im Vakuum. Nach säulenchromatographischer Reinigung (Hexan/EtOAc $=5: 1$ ) erhält man das gewünschte Produkt 70 (49.4 mg, $0.15 \mathrm{mmol}, 66 \%)$ als farbloses Öl: $[\alpha]^{20}{ }_{D}+156.5$ (c 1.00, $\left.\mathrm{CHCl}_{3}\right) ;{ }^{1} \mathrm{H}-\mathrm{NMR}\left(400 \mathrm{MHz}, \mathrm{CDCl}_{3}\right) \delta 7.39-7.27(\mathrm{~m}, 5 \mathrm{H}), 5.65(\mathrm{~d}, \mathrm{~J}=10.6$ $\mathrm{Hz}, 1 \mathrm{H}), 5.59$ (ddd, $J=9.8,5.38,1.96 \mathrm{~Hz}, 1 \mathrm{H}), 5.38(\mathrm{~d}, J=5.1 \mathrm{~Hz}, 1 \mathrm{H}), 4.58(\mathrm{~d}, J=$ $11.9 \mathrm{~Hz}, 1 \mathrm{H}), 4.54(\mathrm{~d}, J=11.9 \mathrm{~Hz}, 1 \mathrm{H}), 3.80(\mathrm{dd}, J=11.7,4.6 \mathrm{~Hz}, 1 \mathrm{H}), 3.61(\mathrm{t}, J=$ $11.7 \mathrm{~Hz}, 1 \mathrm{H}), 3.36(\mathrm{dd}, J=8.6,4.4 \mathrm{~Hz}, 1 \mathrm{H}), 3.29(\mathrm{t}, J=8.6 \mathrm{~Hz}, 1 \mathrm{H}), 2.73-2.67(\mathrm{~m}$, $1 \mathrm{H}), 2.27-2.17(\mathrm{~m}, 1 \mathrm{H}), 2.16-2.06(\mathrm{~m}, 2 \mathrm{H}), 2.03-1.91(\mathrm{~m}, 2 \mathrm{H}), 1.72-1.63(\mathrm{~m}, 1 \mathrm{H}), 1.64$ (s, 3H), 1.57 (br s, 1H), 1.43 (dt, $J=11.4,4.1 \mathrm{~Hz}, 1 \mathrm{H}), 0.90(\mathrm{~d}, J=6.8 \mathrm{~Hz}, 3 \mathrm{H}), 0.79$ $(\mathrm{d}, J=7.2 \mathrm{~Hz}, 3 \mathrm{H}) ;{ }^{13} \mathrm{C}-\mathrm{NMR}\left(100 \mathrm{MHz} \mathrm{CDCl}_{3}\right) \delta 137.75,132.72,131.92,128.48$, 128.33, 127.85, 127.83, 125.93, 75.96, 73.44, 61.35, 40.27, 38.29, 37.43, 34.95, $31.78,30.80,30.57,23.35,16.37$, 14.38; HRMS berechnet für $\mathrm{C}_{23} \mathrm{H}_{32} \mathrm{O}_{2}: 340.2402$, gefunden 340.2400 . 


\section{Darstellung von Benzylether 71}

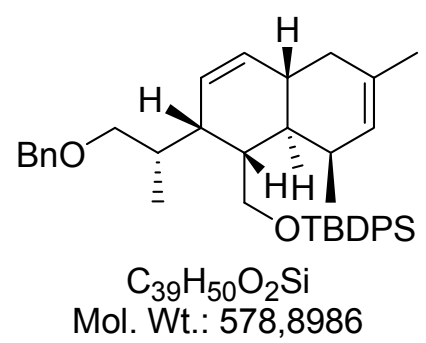

71

TBDPSCl (45 $\mu \mathrm{L}, 176.2 \mu \mathrm{mol})$ wird bei Raumtemperatur langsam zu einer Lösung des Alkohols 70 (30 mg, $88.11 \mu \mathrm{mol}$ ) und Imidazol (19.2 mg, $281.95 \mu \mathrm{mol}$ ) in $\mathrm{CH}_{2} \mathrm{Cl}_{2}$ $(1.0 \mathrm{~mL})$ getropft. Nach 15 Minuten ist die Reaktion beendet und wird mit Wasser $(0.5 \mathrm{~mL})$ gequencht. Die Phasen werden getrennt und man extrahiert die wässrige mit $\mathrm{CH}_{2} \mathrm{Cl}_{2}$. Anschließend trocknet man die gesammelten organischen Phasen mit $\mathrm{MgSO}_{4}$, filtriert den Feststoff ab und entfernt das Lösungsmittel im Vakuum. Nach säulenchromatographischer Reinigung (Hexan/EtOAc $=20: 1$ ) erhält man das gewünschte Produkt 71 (50.4 mg, $87.1 \mu \mathrm{mol}, 99 \%)$ als farbloses Öl: $[\alpha]^{20}+73.6$ (c 1.00, $\left.\mathrm{CHCl}_{3}\right) ;{ }^{1} \mathrm{H}-\mathrm{NMR}\left(400 \mathrm{MHz}, \mathrm{CDCl}_{3}\right) \delta 7.77-7.68(\mathrm{~m}, 4 \mathrm{H}), 7.49-7.33(\mathrm{~m}$, $11 \mathrm{H}), 5.69(\mathrm{~d}, J=10.0 \mathrm{~Hz}, 1 \mathrm{H}), 5.61$ (ddd, $J=10.0,7.8,5.8 \mathrm{~Hz}, 1 \mathrm{H}), 5.26(\mathrm{~d}, J=$ $4.8 \mathrm{~Hz}, 1 \mathrm{H}), 4.56(\mathrm{~s}, 2 \mathrm{H}), 3.67(\mathrm{dd}, J=10.6,4.4 \mathrm{~Hz}, 1 \mathrm{H}), 3.67(\mathrm{t}, J=10.6 \mathrm{~Hz}, 1 \mathrm{H})$, $3.54(\mathrm{dd}, J=8.7,6.5 \mathrm{~Hz}, 1 \mathrm{H}), 3.39(\mathrm{t}, J=8.7 \mathrm{~Hz}, 1 \mathrm{H}), 2.87(\mathrm{~s}, 1 \mathrm{H}), 2.59-2.49(\mathrm{~m}$, $1 \mathrm{H}), 2.19-2.06(\mathrm{~m}, 1 \mathrm{H}), 2.05-1.87(\mathrm{~m}, 3 \mathrm{H}), 1.73-1.64(\mathrm{~m}, 1 \mathrm{H}), 1.62(\mathrm{~s}, 3 \mathrm{H})$, $1.58-1.46(\mathrm{~m}, 1 \mathrm{H}), 1.08(\mathrm{~s}, 9 \mathrm{H}), 0.90(\mathrm{~d}, J=6.8 \mathrm{~Hz}, 3 \mathrm{H}), 0.52(\mathrm{~d}, J=6.8 \mathrm{~Hz}, 3 \mathrm{H})$; ${ }^{13} \mathrm{C}-\mathrm{NMR}\left(100 \mathrm{MHz}, \mathrm{CDCl}_{3}\right) \delta 139.08,135.69,135.63,135.59,133.97,133.87$, $132.52,132.46,129.57,129.56,128.26,127.65,127.60,127.44,127.26,126.29$, 75.98, 72.87, 62.94, 39.92, 38.46, 37.42, 36.28, 32.04, 31.32, 30.70, 26.90, 23.29, 19.27, 15.45, 13.80; HRMS berechnet für $\mathrm{C}_{35} \mathrm{H}_{41} \mathrm{O}_{2} \mathrm{Si}_{1}\left(\mathrm{C}_{39} \mathrm{H}_{50} \mathrm{O}_{2} \mathrm{Si}_{1}\right.$ minus $t$-Butyl): 521.2451 , gefunden 521.2451 . 


\section{Darstellung von Alkohol 72}

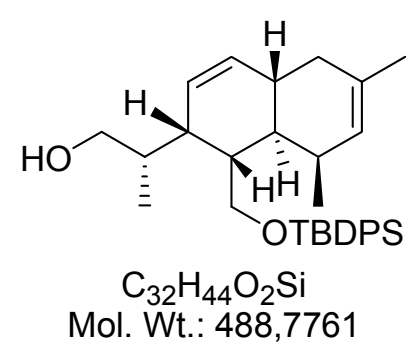

72

Zu einer Lösung des Benzylethers $71(22.6 \mathrm{mg}, 39.0 \mu \mathrm{mol})$ in $\mathrm{CH}_{2} \mathrm{Cl}_{2}(2 \mathrm{~mL})$ wird bei Raumtemperatur $\mathrm{BCl}_{3}{ }^{*} \mathrm{SMe}_{2}\left(2 \mathrm{M}\right.$ in $\mathrm{CH}_{2} \mathrm{Cl}_{2}, 390 \mu \mathrm{L}, 780.66 \mu \mathrm{mol}$ ) langsam zugetropft. Nach drei Stunden ist die Reaktion vollständig. Man quencht mit gesättigter, wässriger $\mathrm{NaHCO}_{3}$-Lösung und extrahiert die wässrige Phase mit MTBE. Anschließend trocknet man die gesammelten organischen Phasen mit $\mathrm{MgSO}_{4}$, filtriert den Feststoff ab und entfernt das Lösungsmittel im Vakuum. Nach säulenchromatographischer Reinigung (Hexan/EtOAc $=10: 1$ ) erhält man das gewünschte Produkt 72 (18.4 mg, $37.9 \mu \mathrm{mol}, 97 \%)$ als farbloses Öl: $[\alpha]^{20}+50.4$ (c 1.00, $\left.\mathrm{CHCl}_{3}\right) ;{ }^{1} \mathrm{H}-\mathrm{NMR}\left(400 \mathrm{MHz}, \mathrm{CDCl}_{3}\right) \delta 7.77-7.66(\mathrm{~m}, 4 \mathrm{H}), 7.48-7.34(\mathrm{~m}$, $6 \mathrm{H}), 5.67(\mathrm{~d}, J=10.2 \mathrm{~Hz}, 1 \mathrm{H}), 5.62$ (ddd, $J=10.2,4.9,2.0 \mathrm{~Hz}, 1 \mathrm{H}), 5.2(\mathrm{~d}, J=$ $5.1 \mathrm{~Hz}, 1 \mathrm{H}), 3.83(\mathrm{dd}, J=10.6,4.4 \mathrm{~Hz}, 1 \mathrm{H}), 3.72-3.57(\mathrm{~m}, 2 \mathrm{H}), 3.50(\mathrm{dd}, J=10.4$, $6.7 \mathrm{~Hz}, 1 \mathrm{H}), 2.82(\mathrm{~s}, 1 \mathrm{H}), 2.25-2.06(\mathrm{~m}, 3 \mathrm{H}), 2.01-1.84(\mathrm{~m}, 3 \mathrm{H}), 1.70-1.61(\mathrm{~m}$, $1 \mathrm{H}), 1.60(\mathrm{~s}, 3 \mathrm{H}), 1.51-1.41(\mathrm{~m}, 1 \mathrm{H}), 1.07(\mathrm{~s}, 9 \mathrm{H}), 0.83(\mathrm{~s}, 3 \mathrm{H}), 0.46(\mathrm{~s}, 3 \mathrm{H})$; ${ }^{13} \mathrm{C}-\mathrm{NMR}\left(100 \mathrm{MHz}, \mathrm{CDCl}_{3}\right) \delta 135.72,135.57,135.19,134.81,133.72,133.51$, 132.58, 132.37, 129.78, 128.19, 127.72, 126.08, 68.58, 62.97, 39.88, 38.34, 37.37, 35.71, 34.61, 31.42, 30.60, 26.93, 26.57, 23.32, 19.23, 19.03; HRMS berechnet für $\mathrm{C}_{28} \mathrm{H}_{35} \mathrm{O}_{2} \mathrm{Si}_{1}\left(\mathrm{C}_{32} \mathrm{H}_{44} \mathrm{O}_{2} \mathrm{Si}_{1}\right.$ minus $t$-Butyl): 431.3111, gefunden 431.3111. 


\section{Darstellung von Aldehyd 73}

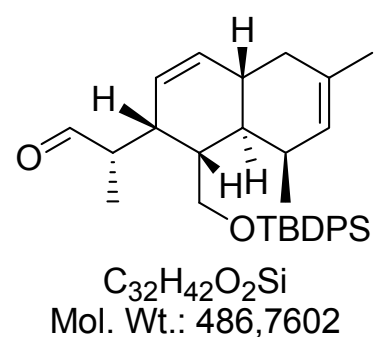

73

Zu einer Lösung von Oxalylchlorid $(13 \mu \mathrm{L}, 147.3 \mu \mathrm{mol})$ in $\mathrm{CH}_{2} \mathrm{Cl}_{2}(0.34 \mathrm{~mL})$ wird bei -78 ${ }^{\circ} \mathrm{C}$ DMSO (16 $\left.\mu \mathrm{L}, 221.0 \mu \mathrm{mol}\right)$ gegeben. 30 Minuten später wird der Alkohol 72 (36 mg, $73.7 \mu \mathrm{mol})$ in $\mathrm{CH}_{2} \mathrm{Cl}_{2}(0.1 \mathrm{~mL})$ hinzugefügt. Nach 15 Minuten erwärmt man auf $-45{ }^{\circ} \mathrm{C}$ und rührt eine Stunde bei dieser Temperatur, bevor man $\mathrm{Et}_{3} \mathrm{~N}(72 \mu \mathrm{L}$, $515.57 \mu \mathrm{mol}$ ) zugibt und dann auf Raumtemperatur erwärmt. Die Reaktionsmischung wird anschließend auf eine Mischung aus MTBE (2 mL) und gesättigter, wässriger $\mathrm{NH}_{4} \mathrm{Cl}$-Lösung (1 mL) gegeben. Die wässrige Phase wird mit MTBE extrahiert. Dann wäscht man die gesammelten organischen Phasen mit gesättigter, wässriger $\mathrm{NaHCO}_{3}$ - und $\mathrm{NaCl}$-Lösung und trocknet sie mit $\mathrm{MgSO}_{4}$. Der Feststoff wird abfiltriert und das Lösungsmittel im Vakuum entfernt. Der Aldehyd 73 wird ohne weitere Reinigungsschritte in die nächste Reaktion eingesetzt: HRMS berechnet für $\mathrm{C}_{28} \mathrm{H}_{33} \mathrm{O}_{2} \mathrm{Si}_{1}\left(\mathrm{C}_{32} \mathrm{H}_{42} \mathrm{O}_{2} \mathrm{Si}_{1}\right.$ minus $t$-Butyl): 429.2954, gefunden 429.2955. 


\section{Darstellung von Methylester 74}

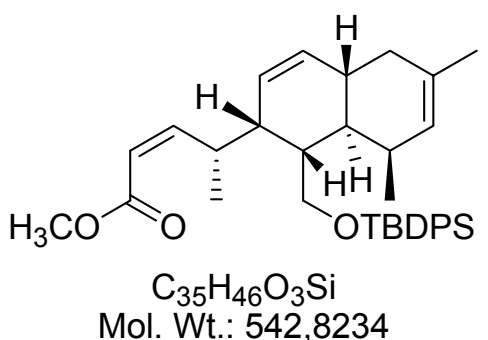

74

Zu einer Lösung von 18-Krone-6 (39 mg, $147.9 \mu \mathrm{mol})$ in THF $(0.7 \mathrm{~mL})$ wird bei $-40{ }^{\circ} \mathrm{C}$ tropfenweise [Bis-(2,2,2-trifluorethoxy)-phosphoryl]-essigsäuremethylester $(20 \mu \mathrm{L}$, $96.1 \mu \mathrm{mol})$ in THF $(0.2 \mathrm{~mL})$ gegeben. Dann wird die Reaktionsmischung auf $-78{ }^{\circ} \mathrm{C}$ abgekühlt und tropfenweise mit KHMDS (0.5 M in Toluol, $177 \mu \mathrm{L}, 88.75 \mu \mathrm{mol})$ versetzt. Nach 15 Minuten wird der Aldehyd 73 (36 mg, $73.96 \mu \mathrm{mol})$ gelöst in THF (0.64 mL) zugegeben. Das Reaktionsgemisch wird innerhalb von drei Stunden auf Raumtemperatur erwärmt. Dann ist die Reaktion beendet und wird durch Zugabe von gesättigter, wässriger $\mathrm{NaHCO}_{3}$-Lösung $(0.8 \mathrm{~mL})$ gequencht. Die Phasen werden getrennt, die wässrige wird mit MTBE extrahiert $(5 \times 15 \mathrm{~mL})$. Die gesammelten organischen Phasen werden mit $\mathrm{MgSO}_{4}$ getrocknet. Anschließend wird der Feststoff abfiltriert und das Lösungsmittel im Vakuum entfernt. Nach säulenchromatographischer Reinigung (Hexan/EtOAc $=20: 1$ ) erhält man das gewünschte Produkt 74 (30.5 mg, $56.2 \mu \mathrm{mol}, 76 \%)$ als farbloses Öl: $[\alpha]^{20}+38.6$ (c 1.00, $\left.\mathrm{CHCl}_{3}\right) ;{ }^{1} \mathrm{H}-\mathrm{NMR}\left(400 \mathrm{MHz}, \mathrm{CDCl}_{3}\right) \delta 7.83-7.63(\mathrm{~m}, 4 \mathrm{H}), 7.54-7.33(\mathrm{~m}$, $6 \mathrm{H}), 6.55$ (dd, $J=11.5,10.04 \mathrm{~Hz}, 1 \mathrm{H}), 5.83$ (ddd, $J=10.1,5.1,2.1 \mathrm{~Hz}, 1 \mathrm{H}), 5.70$ (d, $J=10.1 \mathrm{~Hz}, 1 \mathrm{H}), 5.67(\mathrm{dd}, J=11.5,0.9 \mathrm{~Hz}, 1 \mathrm{H}), 5.26(\mathrm{~d}, J=1.9 \mathrm{~Hz}, 1 \mathrm{H}), 4.04-3.83$ $(\mathrm{m}, 1 \mathrm{H}), 3.81-3.65(\mathrm{~m}, 2 \mathrm{H}), 3.68(\mathrm{~s}, 3 \mathrm{H}), 2.72-2.55(\mathrm{~s}, 1 \mathrm{H}), 2.27-1.82(\mathrm{~m}, 5 \mathrm{H})$, $1.81-1.67(\mathrm{~m}, 1 \mathrm{H}), 1.63(\mathrm{~s}, 3 \mathrm{H}), 1.09(\mathrm{~d}, J=7.8 \mathrm{~Hz}, 3 \mathrm{H}), 1.07$ (s, 9H), 0.43 (d, $J=$ $6.8 \mathrm{~Hz}, 3 \mathrm{H}) ;{ }^{13} \mathrm{C}-\mathrm{NMR}\left(100 \mathrm{MHz}, \mathrm{CDCl}_{3}\right) \delta 165.31,141.42,137.32,137.02,135.67$, 135.02, 134.21, 133.89, 131.34, 131.11, 129.77, 128.95, 128.21, 127.97, 127.36, $64.51,61.33,41.89,41.60,39.66,38.63,32.89,32.57,32.25,28.43,24.61,20.69$, 20.64, 15.14; HRMS berechnet für $\mathrm{C}_{31} \mathrm{H}_{37} \mathrm{O}_{3} \mathrm{Si}_{1} \quad\left(\mathrm{C}_{35} \mathrm{H}_{46} \mathrm{O}_{3} \mathrm{Si}_{1}\right.$ minus $t$-Butyl): 485.3216, gefunden 485.3216. 


\section{Darstellung von Allylalkohol 75}

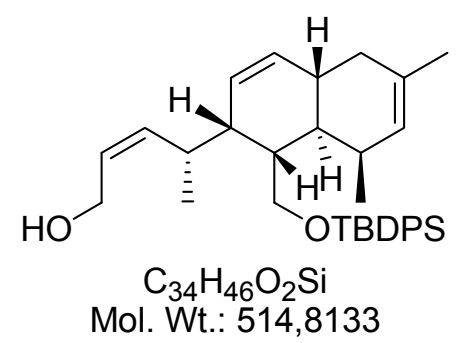

75

Zu einer Lösung des Methylesters $74(11 \mathrm{mg}, 20.3 \mu \mathrm{mol})$ in THF (60 $\mu \mathrm{L})$ wird bei $-50{ }^{\circ} \mathrm{C}$ DiBAl-H (1.5 M in Toluol, $\left.40 \mu \mathrm{L}, 60.8 \mu \mathrm{mol}\right)$ gegeben. Die Lösung wird für drei Stunden bei dieser Temperatur gerührt und dann mit MTBE $(0.24 \mathrm{~mL})$ verdünnt und auf Raumtemperatur erwärmt. Nach der Zugabe von Wasser $(11 \mu \mathrm{L})$ wird die Reaktionsmischung stark gerührt, bis ein weißes Gel entsteht. Zu diesem Gel wird $4 \mathrm{M} \mathrm{NaOH}(11 \mu \mathrm{L})$ und Wasser $(22 \mu \mathrm{L})$ gegeben. Das Reaktionsgemisch wird solange gerührt, bis ein weißer Feststoff entstanden ist. Man trocknet mit $\mathrm{MgSO}_{4}$, filtriert die Feststoffe ab und entfernt das Lösungsmittel im Vakuum. Nach säulenchromato-graphischer Reinigung (Hexan/EtOAc $=10: 2$ ) erhält man das gewünschte Produkt $75(9.0 \mathrm{mg}, 17.4 \mu \mathrm{mol}, 86 \%)$ als farbloses Öl: $[\alpha]^{20}+45.1$ (c 1.00, $\left.\mathrm{CHCl}_{3}\right) ;{ }^{1} \mathrm{H}-\mathrm{NMR}\left(400 \mathrm{MHz}, \mathrm{CDCl}_{3}\right) \delta 7.74-7.68(\mathrm{~m}, 4 \mathrm{H}), 7.50-7.36(\mathrm{~m}$, $6 \mathrm{H}), 5.75$ (ddd, $J=10.0,4.4,2.0 \mathrm{~Hz}, 1 \mathrm{H}), 5.70(\mathrm{~d}, 11.0 \mathrm{~Hz}, 1 \mathrm{H}), 5.67$ (d, $10.0 \mathrm{~Hz}$, $1 \mathrm{H}$ ), 5.47 (dt, $J=11.0,6.3 \mathrm{~Hz}, 1 \mathrm{H}), 5.26(\mathrm{~d}, J=4.9 \mathrm{~Hz}, 1 \mathrm{H}), 4.24$ (ddd, $J=12.6,6.9$, $1.3 \mathrm{~Hz}, 1 \mathrm{H}), 4.15$ (ddd, $J=12.6,6.4,1.3 \mathrm{~Hz}, 1 \mathrm{H}), 3.84(\mathrm{dd}, J=10.5,4.8 \mathrm{~Hz}, 1 \mathrm{H})$, $3.75(\mathrm{t}, J=10.5 \mathrm{~Hz}, 1 \mathrm{H}), 3.09-2.98(\mathrm{~m}, 1 \mathrm{H}), 2.63(\mathrm{~d}, J=2.9 \mathrm{~Hz}, 1 \mathrm{H}), 2.16-2.06$ $(\mathrm{m}, 1 \mathrm{H}), 2.02-1.86(\mathrm{~m}, 3 \mathrm{H}), 1.72-1.64(\mathrm{~m}, 1 \mathrm{H}), 1.62(\mathrm{~s}, 3 \mathrm{H}), 1.55-1.43(\mathrm{~m}, 1 \mathrm{H})$, $1.10(\mathrm{~s}, 9 \mathrm{H}), 0.96(\mathrm{~d}, J=6.8 \mathrm{~Hz}, 3 \mathrm{H}), 0.44(\mathrm{~d}, J=6.7 \mathrm{~Hz}, 3 \mathrm{H}) ;{ }^{13} \mathrm{C}-\mathrm{NMR}(100 \mathrm{MHz}$, $\left.\mathrm{CDCl}_{3}\right) \delta 140.46,137.07,136.95,135.37,135.09,134.10,133.80,131.08,131.00$, 129.65, 129.05, 129.04, 127.90, 127.32, 64.46, 60.33, 41.88, 41.60, 39.76, 38.61, $32.93,32.69,32.21,28.42,24.67,20.68,20.65,15.15$; HRMS berechnet für $\mathrm{C}_{30} \mathrm{H}_{37} \mathrm{O}_{2} \mathrm{Si}_{1}\left(\mathrm{C}_{34} \mathrm{H}_{46} \mathrm{O}_{2} \mathrm{Si}_{1}\right.$ minus $t$-Butyl):457.3267, gefunden 457.3267. 


\section{Darstellung von Allylbromid 76}

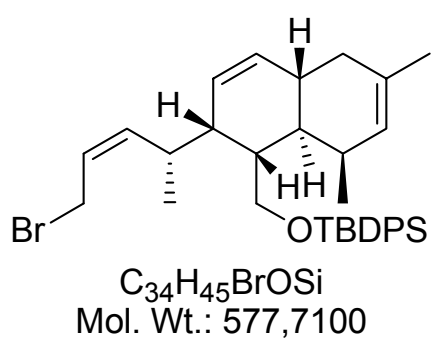

76

Der Allylalkohol 75 (7 mg, $13.7 \mu \mathrm{mol})$ wird in $\mathrm{MeCN}(0.3 \mathrm{~mL})$ gelöst und bei Raumtemperatur nacheinander mit $\mathrm{PPh}_{3}(7.2 \mathrm{mg}, 27.3 \mu \mathrm{mol})$ und $\mathrm{CBr}_{4}(9 \mathrm{mg}, 27.30$ $\mu \mathrm{mol})$ versetzt. Nach 10 Minuten ist die Reaktion beendet und wird mit Wasser (0.1 $\mathrm{mL}$ ) gequencht. Man extrahiert die wässrige Phase mit Hexan. Die gesammelten organischen Phasen werden mit $\mathrm{MgSO}_{4}$ getrocknet und das Lösungsmittel im Vakuum entfernt. Nach säulenchromatographischer Reinigung (Hexan/EtOAc = 20:1) erhält man das gewünschte Produkt 76 (7.2 mg, $12.4 \mu \mathrm{mol}, 91 \%)$ als farbloses Öl: HRMS berechnet für $\mathrm{C}_{30} \mathrm{H}_{36} \mathrm{O}_{1} \mathrm{Si}_{1} \mathrm{Br}_{1} \quad\left(\mathrm{C}_{34} \mathrm{H}_{45} \mathrm{O}_{1} \mathrm{Si}_{1} \mathrm{Br}_{1}\right.$ minus $t$-Butyl): 519.2423 , gefunden 519.2422.

Das Allylbromid 76 wird sofort in die nächste Stufe eingebracht, da es sich innerhalb weniger Stunden zersetzt. 


\section{Darstellung von Phosphoniumsalz 77}

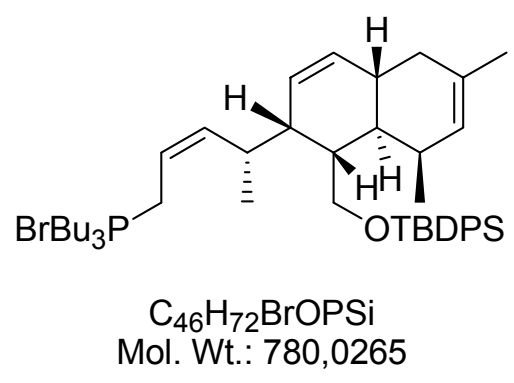

77

Das Bromid 76 (43 mg, $74.4 \mu \mathrm{mol})$ wird in MeCN (0.63 mL) gelöst und bei Raumtemperatur tropfenweise mit Tributylphosphin (28 $\mu \mathrm{L}, 111.7 \mu \mathrm{mol})$ versetzt. Nach zwei Stunden ist die Reaktion beendet. Das Lösungsmittel wird im Vakuum entfernt und das Produkt ohne weitere Reinigung in die nächste Stufe eingesetzt. 


\section{Darstellung von Polyen 82}

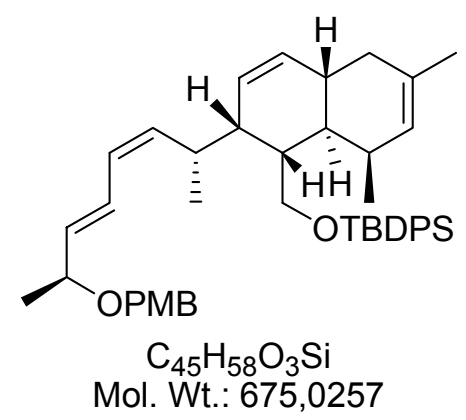

82

Das Phosphoniumsalz 77 wird in Toluol $(0.74 \mathrm{~mL})$ gelöst und bei Raumtemperatur

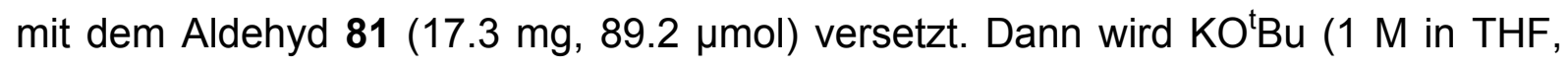
$0.1 \mathrm{ml}, 101.3 \mu \mathrm{mol})$ langsam zu dem Reaktionsgemisch getropft. Nach 45 Minuten ist die Reaktion beendet und wird mit Wasser $(1.5 \mathrm{~mL})$ gequencht. Die wässrige Phase wird mit MTBE extrahiert. Die gesammelten organischen Phasen werden mit $\mathrm{MgSO}_{4}$ getrocknet. Anschließend wird der Feststoff abfiltriert und das Lösungsmittel im Vakuum entfernt. Nach säulenchromatographischer Reinigung (Hexan/EtOAc = 10:2) erhält man das gewünschte Produkt 82 (34.7 mg, $51.4 \mu \mathrm{mol}, 69 \%$ über 2 Stufen) als farbloses Öl: $[\alpha]^{20}{ }_{D}+77.2\left(\right.$ c $\left.1.00, \mathrm{CHCl}_{3}\right) ;{ }^{1} \mathrm{H}-\mathrm{NMR}\left(400 \mathrm{MHz}, \mathrm{CDCl}_{3}\right) \delta 7.72-7.64$ (m, 4H), $7.48-7.36(\mathrm{~m}, 6 \mathrm{H}), 7.29(\mathrm{~d}, J=8.6 \mathrm{~Hz}, 2 \mathrm{H}), 6.90(\mathrm{~d}, J=8.6 \mathrm{~Hz}, 2 \mathrm{H}), 6.18$ (ddd, $J=15.0,14.5,4.4 \mathrm{~Hz}, 1 \mathrm{H}) 5.98(\mathrm{ddd}, J=14.5,9.6,6.7 \mathrm{~Hz}, 1 \mathrm{H}), 5.79$ (dd, $J=$ 15.0, $7.5 \mathrm{~Hz}, 1 \mathrm{H}$ ), 5.73 (dq, $J=9.6,2.1 \mathrm{~Hz}, 1 \mathrm{H}$ ), 5.67 (d, $J=9.6 \mathrm{~Hz}, 1 \mathrm{H}$ ), 5.51 (dd, $J=15.2,9.6 \mathrm{~Hz}, 1 \mathrm{H}), 5.12(\mathrm{~s}, 1 \mathrm{H}), 4.52(\mathrm{~d}, J=11.3 \mathrm{~Hz}, 1 \mathrm{H}), 4.33(\mathrm{~d}, J=11.3 \mathrm{~Hz}$, $1 \mathrm{H}), 4.01-3.92(\mathrm{~m}, 1 \mathrm{H}), 3.82(\mathrm{~s}, 3 \mathrm{H}), 3.79-3.65(\mathrm{~m}, 2 \mathrm{H}), 2.64-2.51(\mathrm{~m}, 1 \mathrm{H})$, $2.43-2.34(\mathrm{br} \mathrm{s}, 1 \mathrm{H}), 2.06-1.77(\mathrm{~m}, 5 \mathrm{H}), 1.77(\mathrm{~s}, 3 \mathrm{H}), 1.31(\mathrm{~d}, J=6.1 \mathrm{~Hz}, 3 \mathrm{H})$, $1.1-0.98(\mathrm{~m}, 1 \mathrm{H}), 1.07(\mathrm{~s}, 9 \mathrm{H}), 1.03(\mathrm{~d}, J=6.8 \mathrm{~Hz}, 3 \mathrm{H}), 0.86(\mathrm{~d}, J=0.86 \mathrm{~Hz}, 3 \mathrm{H})$; ${ }^{13} \mathrm{C}-\mathrm{NMR}\left(100 \mathrm{MHz}, \mathrm{CDCl}_{3}\right) \delta 140.65,135.64,133.91,133.00,132.97,132.58$, $132.19,132.11,131.04,131.00,129.54,129.27,129.25,128.85,128.07,128.03$, $127.64,127.62,127.58,113.75,75.29,69.54,64.85,55.29,45.56,43.87,42.28$, $38.89,36.95,36.40,36.28,35.94,26.95,23.43,22.15,21.71,19.28$; HRMS berechnet für $\mathrm{C}_{41} \mathrm{H}_{49} \mathrm{O}_{3} \mathrm{Si}_{1} \quad\left(\mathrm{C}_{45} \mathrm{H}_{58} \mathrm{O}_{3} \mathrm{Si}_{1}\right.$ minus $t$-Butyl): 617.5234, gefunden 617.5230. 


\section{Darstellung von Alkohol 90}

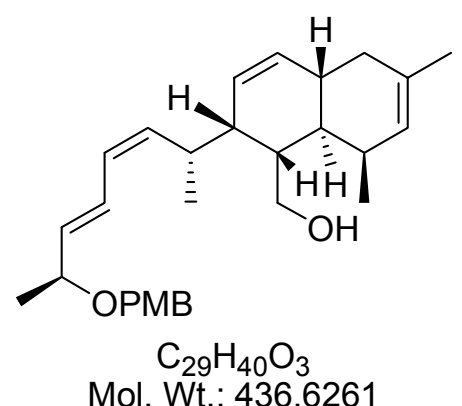

90

Zu einer Lösung von Polyen 82 (50 mg, $74.1 \mu \mathrm{mol})$ in Pyridin $(5 \mathrm{~mL})$ und THF ( $5 \mathrm{~mL}$ ) wird bei Raumtemperatur HF*Pyridin (70\%ig, $2 \mathrm{~mL}$ ) zugegeben. Nach fünf Minuten ist die Reaktion beendet und wird durch die Zugabe von gesättigter, wässriger $\mathrm{NaHCO}_{3}$-Lösung $(2 \mathrm{~mL})$, EtOAc $(4 \mathrm{~mL})$ und pH 7 Phosphatpuffer $(2 \mathrm{~mL})$ gequencht. Die Phasen werden getrennt. Die wässrige Phase wird mit EtOAc ausgeschüttelt. Die gesammelten organischen Phasen trocknet man mit $\mathrm{MgSO}_{4}$, filtriert den Feststoff ab und entfernt das Lösungsmittel im Vakuum. Nach säulenchromatographischer Reinigung (Hexan/EtOAc = 2:1) erhält man das gewünschte Produkt $90(20.38 \mathrm{mg}$, $46.7 \mu \mathrm{mol}, 63 \%)$ als farbloses Öl: $[\mathrm{a}]_{\mathrm{D}}^{20}+73.1$ (c 1.00, $\left.\mathrm{CHCl}_{3}\right) ;{ }^{1} \mathrm{H}-\mathrm{NMR}(400 \mathrm{MHz}$, $\left.\mathrm{CDCl}_{3}\right) \delta 7.39(\mathrm{~d}, J=8.53 \mathrm{~Hz}, 2 \mathrm{H}), 6.93(\mathrm{~d}, J=8.53 \mathrm{~Hz}, 2 \mathrm{H}), 6.34$ (ddd, $J=14.7$, 14.5, $1.3 \mathrm{~Hz}, 1 \mathrm{H}$ ), 6.25 (dd, $J=14.7,10.2 \mathrm{~Hz}, 1 \mathrm{H}$ ), 5.92 (ddd, $J=9.6,4.3,2.0 \mathrm{~Hz}$, $1 \mathrm{H}), 5.83(\mathrm{dd}, J=9.6,6.5 \mathrm{~Hz}, 1 \mathrm{H}), 5.81-5.76(\mathrm{~m}, 1 \mathrm{H}), 5.69$ (ddd, $J=14.5,7.5$, $3.1 \mathrm{~Hz}, 1 \mathrm{H}), 5.32(\mathrm{~s}, 1 \mathrm{H}), 4.64(\mathrm{dd}, J=11.6,6.1 \mathrm{~Hz}, 1 \mathrm{H}), 4.41(\mathrm{~d}, J=11.6 \mathrm{~Hz}, 1 \mathrm{H})$, $4.07-3.95(\mathrm{~m}, 1 \mathrm{H}), 3.72-3.64(\mathrm{~m}, 2 \mathrm{H}), 3.42(\mathrm{~s}, 3 \mathrm{H}), 2.70-2.58(\mathrm{~m}, 1 \mathrm{H}), 2.34$ (br s, 1H), $2.18-1.95(\mathrm{~m}, 4 \mathrm{H}), 1.94-1.83(\mathrm{~m}, 1 \mathrm{H}), 1.75(\mathrm{~s}, 3 \mathrm{H}), 1.41(\mathrm{~d}, J=6.5 \mathrm{~Hz}$, $3 \mathrm{H}), 1.22-1.09(\mathrm{~m}, 1 \mathrm{H}), 1.18(\mathrm{~d}, J=6.5 \mathrm{~Hz}, 3 \mathrm{H}), 1.05(\mathrm{~d}, J=6.8 \mathrm{~Hz}, 3 \mathrm{H}) ;{ }^{13} \mathrm{C}-\mathrm{NMR}$ $\left(100 \mathrm{MHz}, \mathrm{CDCl}_{3}\right) \delta 159.34,139.97,134.12,134.11,133.77,132.38,131.75,131.62$, $130.60,130.50,129.05,128.90,113.81,75.27,69.65,63.93,54.53,45.38,44.83$, $42.75,38.49,36.93,36.86,36.52,23.30,21.75,21.52$; HRMS berechnet für $\mathrm{C}_{29} \mathrm{H}_{40} \mathrm{O}_{3}: 436.2977$, gefunden 436.2979 . 


\section{Darstellung von Aldehyd 91}

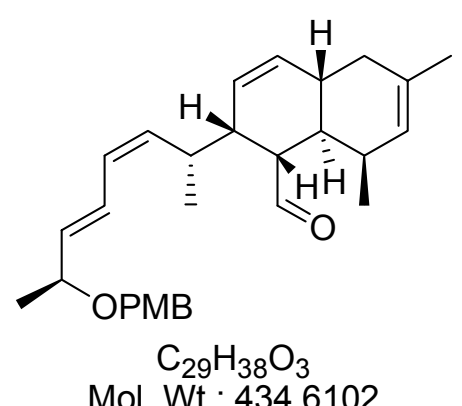

Mol. Wt.: 434,6102

91

$\mathrm{Zu}$ einer Lösung des Alkohols 90 (25 mg, $57.3 \mu \mathrm{mol})$ in $\mathrm{CH}_{2} \mathrm{Cl}_{2}(1 \mathrm{~mL})$ wird bei Raumtemperatur Dess-Martin-Periodinan $(29.09 \mathrm{mg}, 68.7 \mu \mathrm{mol})$ gegeben und das Gemisch für eine Stunde gerührt. Dann gibt man $\mathrm{Na}_{2} \mathrm{~S}_{2} \mathrm{O}_{3}{ }^{*} 5 \mathrm{H}_{2} \mathrm{O}(50 \mathrm{mg})$ gelöst in gesättigter, wässriger $\mathrm{NaHCO}_{3}$-Lösung $(0.5 \mathrm{~mL})$ hinzu und rührt, bis die Lösung klar ist. Danach werden die zwei Phasen getrennt, wobei man die wässrige mit $\mathrm{CH}_{2} \mathrm{Cl}_{2}$ ( $3 \mathrm{x}$ ) extrahiert. Die gesammelten organischen Phasen werden mit gesättigter, wässriger $\mathrm{NaCl}$-Lösung gewaschen und anschließend mit $\mathrm{MgSO}_{4}$ getrocknet. Der Feststoff wird abfiltriert und anschließend wird das Lösungsmittel am Rotationsverdampfer entfernt. Nach säulenchromatographischer Reinigung (Hexan/EtOAc = 10:1) erhält man das gewünschte Produkt 91 (14.43 mg, $33.2 \mu \mathrm{mol}$, $58 \%$ ) als farbloses Öl: $\mathrm{HRMS}$ berechnet für $\mathrm{C}_{29} \mathrm{H}_{38} \mathrm{O}_{3}: 434.2821$, gefunden 434.2823. Der Aldehyd 91 wird sofort in die nächste Reaktion eingesetzt. 


\section{Darstellung von Aldehyd 113}

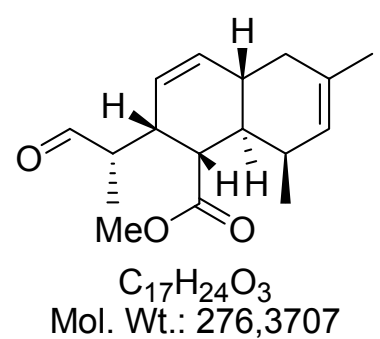

113

Zu dem Lacton 66 (35 mg, $142.7 \mu \mathrm{mol}$ ) wird eine Lösung von $\mathrm{KOH}$ (400 mg) in Methanol (4 mL) gegeben. Nach 30 Minuten ist die Bildung der Säure vollständig und das Methanol wird am Rotationsverdampfer entfernt. Dann löst man den festen Rückstand in Wasser $(25 \mathrm{~mL})$ und säuert die Lösung mit $2 \mathrm{M} \mathrm{HCl}$ an, bis man einen $\mathrm{pH}$-Wert von 4-5 eingestellt hat. Die wässrige Lösung wird mit EtOAc ausgeschüttelt und die gesammelten organischen Phasen werden mit $\mathrm{MgSO}_{4}$ getrocknet. Der Feststoff wird abfiltriert und das EtOAc im Vakuum entfernt. Man versetzt den weißen Rückstand sofort mit einer Diazomethan-Diethylether-Lösung und zwar so lange, bis der Kolbeninhalt die gelbe Farbe des Diazomethans beibehält. Dann gibt man $\mathrm{CH}_{2} \mathrm{Cl}_{2}(1 \mathrm{~mL}) \mathrm{zu}$ und entfernt den Diethylether und das Diazomethan im Vakuum. $\mathrm{Zu}$ dem verbleibenden $\mathrm{CH}_{2} \mathrm{Cl}_{2}$ gibt man Dess-Martin Periodinan (30\%ige Lösung in $\mathrm{CH}_{2} \mathrm{Cl}_{2}, 1 \mathrm{~mL}$ ) und rührt eine halbe Stunde bei Raumtemperatur. Dann gibt man $\mathrm{Na}_{2} \mathrm{~S}_{2} \mathrm{O}_{3}{ }^{*} 5 \mathrm{H}_{2} \mathrm{O}$ (50 mg) gelöst in gesättigter, wässriger $\mathrm{NaHCO}_{3}$-Lösung $(0.5 \mathrm{~mL})$ hinzu und rührt, bis die Lösung klar ist. Danach werden die zwei Phasen getrennt, wobei man die wässrige mit $\mathrm{CH}_{2} \mathrm{Cl}_{2}(3 \mathrm{x})$ extrahiert. Die gesammelten organischen Phasen werden mit gesättigter, wässriger $\mathrm{NaCl}$-Lösung gewaschen und anschließend mit $\mathrm{MgSO}_{4}$ getrocknet. Der Feststoff wird abfiltriert und anschließend wird das Lösungsmittel am Rotationsverdampfer entfernt. Nach säulenchromatographischer Reinigung (Hexan/EtOAc $=10: 1$ ) erhält man das gewünschte Produkt 113 (30.38 mg, $109.9 \mu \mathrm{mol}, 77 \%)$ als farbloses Öl: $[\alpha]^{20}{ }_{D}+46.2$ (c 1.00, $\mathrm{CHCl}_{3}$ ); ${ }^{1} \mathrm{H}-\mathrm{NMR}\left(400 \mathrm{MHz}, \mathrm{CDCl}_{3}\right) \delta 9.70$ (s, $\left.1 \mathrm{H}\right), 5.77$ (dt, $J=9.7,2.0 \mathrm{~Hz}$, 1H), $5.59(\mathrm{dq}, J=9.7,2.3 \mathrm{~Hz}, 1 \mathrm{H}), 5.13(\mathrm{~s}, 1 \mathrm{H}), 3.65(\mathrm{~s}, 3 \mathrm{H}), 3.02-2.93(\mathrm{~m}, 1 \mathrm{H})$, 
$2.8-2.7(\mathrm{~m}, 2 \mathrm{H}), 2.18-2.03(\mathrm{~m}, 3 \mathrm{H}), 1.90(\mathrm{t}, \mathrm{J}=15.4 \mathrm{~Hz}, 1 \mathrm{H}), 1.70(\mathrm{~s}, 3 \mathrm{H}), 1.44(\mathrm{q}$, $J=9.4 \mathrm{~Hz}, 1 \mathrm{H}), 1.16(\mathrm{~d}, J=7.2 \mathrm{~Hz}, 3 \mathrm{H}), 0.92(\mathrm{~d}, J=6.8 \mathrm{~Hz}, 3 \mathrm{H}) ;{ }^{13} \mathrm{C}-\mathrm{NMR}$ $\left(100 \mathrm{MHz}, \mathrm{CDCl}_{3}\right) \delta 203.74,175.75,133.83,132.86,128.82,126.08,51.52,47.90$, 47.71, 43.91, 37.75, 37.65, 37.04, 36.38, 23.16, 20.85, 12.16; HRMS berechnet für $\mathrm{C}_{17} \mathrm{H}_{24} \mathrm{O}_{3}: 276.1725$, gefunden 276.1724. 


\section{Darstellung von Methylester 114}

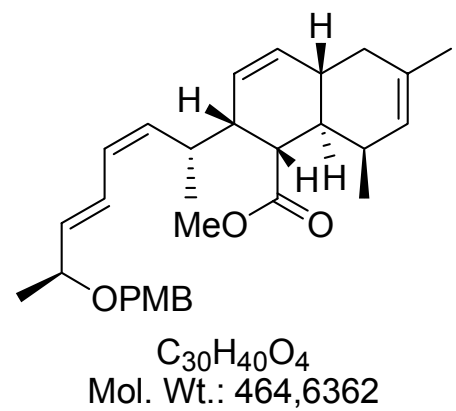

114

Bei $-80{ }^{\circ} \mathrm{C}$ wird der Phosphonoester 89 (128 mg, $\left.285.4 \mu \mathrm{mol}\right)$ in $\mathrm{Et}_{2} \mathrm{O}(0.8 \mathrm{~mL})$ tropfenweise mit KHMDS (0.5 M in Toluol, $0.17 \mathrm{~mL}, 86.84 \mu \mathrm{mol})$ versetzt. Nach 30 Minuten wird der Aldehyd $113(20 \mathrm{mg}, 72.37 \mu \mathrm{mol})$ gelöst in $\mathrm{Et}_{2} \mathrm{O}(0.53 \mathrm{~mL})$ zugegeben. Nach eineinhalb Stunden ist die Reaktion beendet und wird durch Zugabe von gesättigter, wässriger $\mathrm{NaHCO}_{3}$-Lösung $(1.0 \mathrm{~mL})$ gequencht und auf Raumtemperatur erwärmt. Die Phasen werden getrennt, die wässrige wird mit MTBE extrahiert $(5 \times 20 \mathrm{~mL})$. Die gesammelten organischen Phasen werden mit $\mathrm{MgSO}_{4}$ getrocknet. Dann wird der Feststoff abfiltriert und das Lösungsmittel im Vakuum entfernt. Nach säulenchromatographischer Reinigung (Hexan/EtOAc $=20: 1)$ erhält man das gewünschte Produkt $114(18.5 \mathrm{mg}, 39.8 \mu \mathrm{mol}, 55 \%)$ als farbloses Öl: $[\alpha]^{20}$ +65.2 (c 1.00, $\mathrm{CHCl}_{3}$ ); ${ }^{1} \mathrm{H}-\mathrm{NMR}\left(400 \mathrm{MHz}, \mathrm{CDCl}_{3}\right) \delta 7.28$ (d, J = $8.5 \mathrm{~Hz}, 2 \mathrm{H}$ ), 6.90 (d, $J=8.5 \mathrm{~Hz}, 2 \mathrm{H}), 6.19$ (d, $J=15.5,14.8 \mathrm{~Hz}, 1 \mathrm{H}), 6.03$ (dd, $J=14.8,10.4 \mathrm{~Hz}, 1 \mathrm{H}), 5.89$ (d, J = 9.6 Hz, 1H), 5.80 (d, J = 9.6 Hz, 1H), 5.67 (dd, J = 15.5, 7.3 Hz, 1H), 5.55 (dd, $J=15.2,10.4 \mathrm{~Hz}, 1 \mathrm{H}), 5.16(\mathrm{~s}, 1 \mathrm{H}), 4.5(\mathrm{~d}, J=11.4 \mathrm{~Hz}, 1 \mathrm{H}), 4,33(\mathrm{~d}, J=11.4 \mathrm{~Hz}$, 1H), $4.04-3.92(\mathrm{~m}, 1 \mathrm{H}), 3.83(\mathrm{~s}, 3 \mathrm{H}), 3.63(\mathrm{~s}, 3 \mathrm{H}), 2.73-2.64(\mathrm{~m}, 1 \mathrm{H}), 2.51-2.39$ (m, 1H), $2.39-2.23(\mathrm{~m}, 5 \mathrm{H}), 1.69(\mathrm{~s}, 3 \mathrm{H}), 1.33-1.21(\mathrm{~m}, 1 \mathrm{H}), 1.30(\mathrm{~d}, J=5.5 \mathrm{~Hz}$, $3 \mathrm{H}), 1.12(\mathrm{~d}, J=5.5 \mathrm{~Hz}, 3 \mathrm{H}), 1.01(\mathrm{~d}, J=6.8 \mathrm{~Hz}, 3 \mathrm{H}) ;{ }^{13} \mathrm{C}-\mathrm{NMR}\left(100 \mathrm{MHz}, \mathrm{CDCl}_{3}\right)$ $\delta 174.86,159.35,138.71,134.31,134.25,133.05,132.75,131.74,131.67,129.43$, 129.06, 129.02, 128.99, 113.82, 75, 19, 69.58, 54.53, 50.78, 48.97, 46.54, 42.89, $38.87,38.30,37.15,23.05,21.75,20.85,18.20$; HRMS berechnet für $\mathrm{C}_{30} \mathrm{H}_{40} \mathrm{O}_{4}$ : 464.2927, gefunden 464.2927.

Bei dem entstandenen Produkt 114 handelt es sich um ein Z/E:E/E-Gemisch von 3:1. 


\section{Darstellung von Diketoester 107}

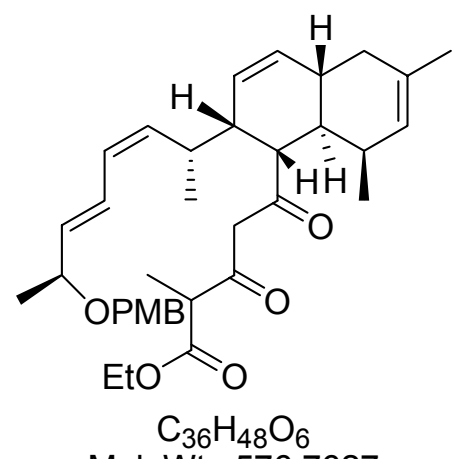

Mol. Wt.: 576,7627

107

Eine Suspension von Natriumhydrid (60\%ige Suspension in Mineralöl, $80 \mathrm{mg}$, $2.0 \mathrm{mmol}$ ) in THF $(3 \mathrm{~mL})$ wird auf $0{ }^{\circ} \mathrm{C}$ abgekühlt und dann mit Ethyl-2methylacetoacetat (109) $(0.32 \mathrm{~mL}, 2.0 \mathrm{mmol})$ in THF $(2.3 \mathrm{~mL})$ versetzt. Dann wird das Eisbad entfernt und eineinhalb Stunden bei Raumtemperatur gerührt. Anschließend wird die Reaktionsmischung wieder auf $0{ }^{\circ} \mathrm{C}$ abgekühlt und man tropft $n$-BuLi (2.5 M in Hexan, $0.8 \mathrm{~mL}, 2.0 \mathrm{mmol}$ ) zu. Es wird eine dreiviertel Stunde bei $0{ }^{\circ} \mathrm{C}$ und eine halbe Stunde bei Raumtemperatur gerührt, bevor man die Reaktionsmischung auf $-78{ }^{\circ} \mathrm{C}$ abkühlt und den Methylester 114 (46.46 mg, $0.10 \mathrm{mmol})$ in THF $(0.9 \mathrm{~mL})$ zugibt. Man rührt eine Stunde bei $0{ }^{\circ} \mathrm{C}$ und lässt dann langsam auf Raumtemperatur erwärmen. Wenn der Methylester 114 vollständig abreagiert ist (ca. nach zwei Stunden bei Raumtemperatur), wird $1 \mathrm{~N} \mathrm{H}_{2} \mathrm{SO}_{4}(1 \mathrm{~mL})$ zugegeben. Die Phasen werden getrennt, die wässrige wird mit MTBE extrahiert (5 $\mathrm{x}$ $2 \mathrm{~mL}$ ). Die gesammelten organischen Phasen werden mit Wasser, gesättigter, wässriger $\mathrm{NaHCO}_{3}$-Lösung und gesättigter, wässriger $\mathrm{NaCl}$-Lösung gewaschen und anschließend mit $\mathrm{MgSO}_{4}$ getrocknet. Der Feststoff wird abfiltriert und das Lösungsmittel im Vakuum entfernt. Nach säulenchromatographischer Reinigung $($ Hexan/EtOAc $=10: 1)$ erhält man das gewünschte Produkt 107, das allerdings noch sehr stark mit Ethyl-2-methylacetoacetat (109) verunreinigt ist. Da dieses chromatographisch nicht abzutrennen ist, wird die Mischung in die nächste Stufe eingesetzt; HRMS berechnet für $\mathrm{C}_{36} \mathrm{H}_{48} \mathrm{O}_{6}$ : 576.3451 , gefunden 576.3451 . 


\section{Darstellung von Ethylester 79}

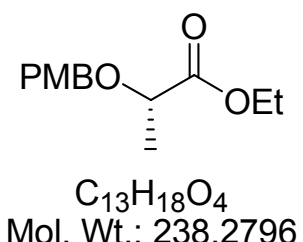

79

Zu einer Mischung von (S)-(-)-Milchsaeureethylester (78) (7 g, $59.32 \mathrm{mmol}$ ) und (4-Methoxybenzyl)-trichloracetimidat $(25.13 \mathrm{~g}, 88.9 \mathrm{mmol})$ in $\mathrm{CH}_{2} \mathrm{Cl}_{2}(170 \mathrm{~mL}$ ) wird Camphersulfonsäure $(1.38 \mathrm{~g}, 5.9 \mathrm{mmol})$ gegeben. Anschließend lässt man das Reaktionsgemisch 16 Stunden lang rühren. Danach filtriert man den entstandenen Niederschlag ab, wäscht mit gesättigter, wässriger $\mathrm{NaHCO}_{3}$-Lösung, extrahiert mit MTBE und trocknet die gesammelten organischen Phasen über $\mathrm{MgSO}_{4}$. Das Lösungsmittel wird im Vakuum entfernt. Danach wird Hexan zu dem Rückstand gegeben, sodass sich erneut ein Niederschlag bildet. Dieser wird ebenfalls abfiltriert. Nach Entfernung des Lösungsmittels im Vakuum und säulenchromatographischer Reinigung (Hexan/EtOAc $=8: 1)$ erhält man das gewünschte Produkt 79 (13.7 g,

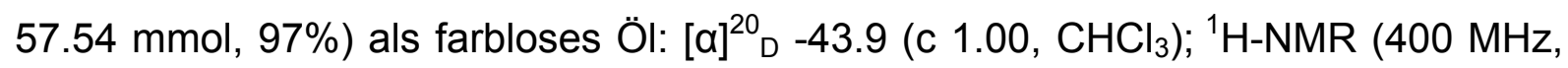
$\left.\mathrm{CDCl}_{3}\right) \delta 7.28(\mathrm{~d}, J=8.5 \mathrm{~Hz}, 2 \mathrm{H}), 6.91(\mathrm{~d}, J=8.5 \mathrm{~Hz}, 2 \mathrm{H}), 4.64(\mathrm{~d}, J=11.29 \mathrm{~Hz}, 1 \mathrm{H})$, $4.12(\mathrm{q}, J=7.2 \mathrm{~Hz}, 2 \mathrm{H}), 4.44(\mathrm{~d}, J=11.29 \mathrm{~Hz}, 1 \mathrm{H}), 3.86(\mathrm{~s}, 3 \mathrm{H}), 3.50-3.41$ (m, $1 \mathrm{H}), 1.13(\mathrm{~d}, J=6.09 \mathrm{~Hz}, 3 \mathrm{H}), 1.30(\mathrm{t}, J=7.2 \mathrm{~Hz}, 3 \mathrm{H}) ;{ }^{13} \mathrm{C}-\mathrm{NMR}\left(100 \mathrm{MHz}, \mathrm{CDCl}_{3}\right)$ $\delta 172.01,161.13,129.56,128.93,114.04,78.54,72.66,59.83,56.02,15.90,13.61$; HRMS berechnet für $\mathrm{C}_{13} \mathrm{H}_{18} \mathrm{O}_{4}: 238.1205$, gefunden 238.1204. 


\section{Darstellung von Alkohol 80}

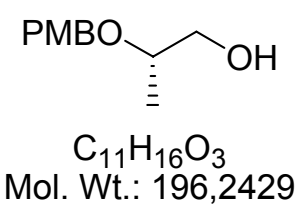

80

Zum Ester 79 (10 g, $41.97 \mathrm{mmol})$ gelöst in $\mathrm{CH}_{2} \mathrm{Cl}_{2}(126 \mathrm{~mL})$ wird bei $-78{ }^{\circ} \mathrm{C}$ tropfenweise DiBAl-H (1.5 M in Toluol, $84 \mathrm{~mL}, 125.90 \mathrm{mmol}$ ) gegeben. Anschließend wird eineinhalb Stunden bei $-78{ }^{\circ} \mathrm{C}$ gerührt. Dann wird die Reaktionsmischung mit MTBE (400 mL) versetzt und auf Raumtemperatur erwärmt. Nach einer Stunde Rühren bei Raumtemperatur wird langsam Wasser $(11.5 \mathrm{~mL})$ zugetropft. Dabei entsteht nach ca. fünf Minuten ein weißes Gel, welches durch Auflockern mit einem Spatel wieder zum Rühren gebracht werden kann. Dann addiert man $4 \mathrm{~N}$ Natronlauge $(11.5 \mathrm{~mL})$ und Wasser $(23 \mathrm{~mL})$. Anschließend lässt man das Reaktionsgemisch rühren, bis ein weißer Feststoff entsteht. Nach Zugabe von $\mathrm{MgSO}_{4}$ wird der gesamte Feststoff abfiltriert und das Lösungsmittel am Rotationsverdampfer entfernt. Nach säulenchromatographischer Reinigung (Hexan/EtOAc = $5: 1)$ erhält man das gewünschte Produkt $80(7.08 \mathrm{~g}, 36.09 \mathrm{mmol}$, 86\%) als farbloses Öl: [a $]^{20}-41.2$ (c 1.00, $\mathrm{CHCl}_{3}$ ); ${ }^{1} \mathrm{H}-\mathrm{NMR}\left(400 \mathrm{MHz}, \mathrm{CDCl}_{3}\right) \delta 7.29$ (d, $J=8.5 \mathrm{~Hz}, 2 \mathrm{H}), 6.90(\mathrm{~d}, J=8.5 \mathrm{~Hz}, 2 \mathrm{H}), 4.60(\mathrm{~d}, J=11.26 \mathrm{~Hz}, 1 \mathrm{H}), 4.44$ (d, $J=$ $11.26 \mathrm{~Hz}, 1 \mathrm{H}), 3.83(\mathrm{~s}, 3 \mathrm{H}), 3.72-3.58(\mathrm{~m}, 2 \mathrm{H}), 3.54-3.46(\mathrm{~m}, 1 \mathrm{H}), 2.12$ (br s, $1 \mathrm{H}), 1.19(\mathrm{~d}, J=6.14 \mathrm{~Hz}, 3 \mathrm{H}) ;{ }^{13} \mathrm{C}-\mathrm{NMR}\left(100 \mathrm{MHz}, \mathrm{CDCl}_{3}\right) \quad \delta 159.24,130.57$, $129.37,114.01,75.28,70.51,66.40,55.37,15.89$; HRMS berechnet für $\mathrm{C}_{11} \mathrm{H}_{16} \mathrm{O}_{3}$ : 196.1099, gefunden 196.1099. 


\section{Darstellung von Aldehyd 81}

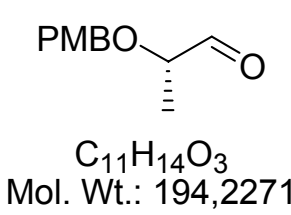

81

Zu einer Lösung von Oxalylchlorid $(0.56 \mathrm{~mL}, 6.38 \mathrm{mmol})$ in $\mathrm{CH}_{2} \mathrm{Cl}_{2}(14.9 \mathrm{~mL})$ wird bei $-78{ }^{\circ} \mathrm{C}$ DMSO $(0.68 \mathrm{~mL}, 9.57 \mathrm{mmol})$ gegeben. 30 Minuten später wird der Alkohol 80 (626 mg, $3.19 \mathrm{mmol})$ in $\mathrm{CH}_{2} \mathrm{Cl}_{2}(4.5 \mathrm{~mL})$ addiert. Nach 15 Minuten erwärmt man auf $-45{ }^{\circ} \mathrm{C}$ und rührt eine Stunde bei dieser Temperatur. Dann wird $\mathrm{Et}_{3} \mathrm{~N}$ (3.11 mL, $22.33 \mathrm{mmol}$ ) zu dem Reaktionsgemisch gegeben und auf Raumtemperatur erwärmt. Die Reaktionsmischung wird anschließend auf eine Mischung aus MTBE (70 mL) und $\mathrm{NH}_{4} \mathrm{Cl}$ (35 mL) gegeben. Die wässrige Phase wird mit MTBE extrahiert. Dann wäscht man die gesammelten organischen Phasen mit gesättigter, wässriger $\mathrm{NaHCO}_{3}$ - und $\mathrm{NaCl}$-Lösung und trocknet sie mit $\mathrm{MgSO}_{4}$. Der Feststoff wird abfiltriert und das Lösungsmittel im Vakuum entfernt. Das Produkt 81 wird ohne weitere Reinigungsschritte sofort in die nächste Reaktion eingesetzt: HRMS berechnet für $\mathrm{C}_{11} \mathrm{H}_{14} \mathrm{O}_{3}:$ 194.0943, gefunden 194.0944. 


\section{Darstellung von Alken 88}

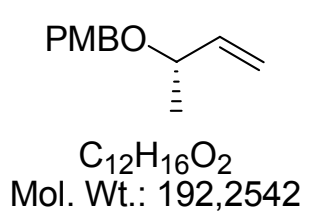

88

Zu einer Suspension von Methyltriphenylphosphoniumbromid $(2.88 \mathrm{~g}, 8.06 \mathrm{mmol})$ in THF (70 mL) wird $n$-BuLi (2.5 M in Hexan, $2.06 \mathrm{~mL}, 5.16 \mathrm{mmol})$ bei $-78{ }^{\circ} \mathrm{C}$ gegeben. Die Reaktionsmischung wird 30 Minuten bei $-78{ }^{\circ} \mathrm{C}$ und 30 Minuten bei Raumtemperatur gerührt. Dann wird sie erneut auf $-78{ }^{\circ} \mathrm{C}$ abgekühlt und der Aldehyd $81(0.63 \mathrm{~g}, 3.19 \mathrm{mmol})$ in THF $(9 \mathrm{~mL})$ zugegeben. Es wird fünf Minuten bei $-78^{\circ} \mathrm{C}$ gerührt und anschließend eine Stunde bei Raumtemperatur. Dann wird gesättigte, wässrige $\mathrm{NH}_{4} \mathrm{Cl}$-Lösung zugegeben $(27.8 \mathrm{~mL} / \mathrm{mmol})$. Die wässrige Phase wird mit MTBE extrahiert. Die gesammelten organischen Phasen werden mit $\mathrm{MgSO}_{4}$ getrocknet. Der Feststoff wird abfiltriert und das Lösungsmittel im Vakuum entfernt. Nach säulenchromatographischer Reinigung (Hexan:EtOAc $=20: 1$ ) erhält man das gewünschte Produkt 88 (0.45 g, $2.33 \mathrm{mmol}, 73 \%$ über 2 Stufen) als farbloses Öl: $[\alpha]^{20}{ }_{D}-29.6$ (c 1.00, $\mathrm{CHCl}_{3}$ ); ${ }^{1} \mathrm{H}-\mathrm{NMR}\left(400 \mathrm{MHz} \mathrm{CDCl}_{3}\right) \delta 7.29$ (d, J = $\left.8.3 \mathrm{~Hz}, 2 \mathrm{H}\right)$, $6.90(\mathrm{~d}, J=8.3 \mathrm{~Hz}, 2 \mathrm{H}), 5.82(\mathrm{ddd}, J=17.3,10.2,7.3 \mathrm{~Hz}, 1 \mathrm{H}), 5.27-5.18(\mathrm{~m}, 2 \mathrm{H})$, $4.53(\mathrm{~d}, J=11.4 \mathrm{~Hz}, 1 \mathrm{H}), 4.35(\mathrm{~d}, J=11.4 \mathrm{~Hz}, 1 \mathrm{H}), 3.97-3.89(\mathrm{~m}, 1 \mathrm{H}), 3.82(\mathrm{~s}, 3 \mathrm{H})$, $1.30(\mathrm{~d}, J=6.1 \mathrm{~Hz}, 3 \mathrm{H}) ;{ }^{13} \mathrm{C}-\mathrm{NMR}\left(100 \mathrm{MHz}, \mathrm{CDCl}_{3}\right) \delta 159.16,140.39,130.94$, 129.37, 115.98, 113.74, 75.94, 69.64, 55.20, 21.33; HRMS berechnet für $\mathrm{C}_{12} \mathrm{H}_{16} \mathrm{O}_{2}$ : 192.1150, gefunden 192.1151. 


\section{Darstellung von Phosphonat 87}

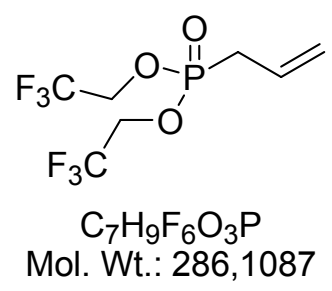

87

Tris(2,2,2-trifluorethyl)phosphit $(0.4 \mathrm{~mL}, 1.52 \mathrm{mmol})$, Allylbromid (0.25 mL, 3.05 $\mathrm{mmol}$ ) und Tetrabutylammoniumiodid (30 mg) werden in einem geschlossenen Gefäß ohne Druckausgleich für 24 Stunden bei $180{ }^{\circ} \mathrm{C}$ erhitzt. Dann wird das überschüssige Allylbromid im Vakuum entfernt. Nach säulenchromatographischer Reinigung (Hexan:EtOAc = 10:2) erhält man das gewünschte Produkt 87 (0.27 g, $0.93 \mathrm{mmol}, 61 \%)$ als farbloses Öl: ${ }^{1} \mathrm{H}-\mathrm{NMR}\left(400 \mathrm{MHz}, \mathrm{CDCl}_{3}\right) \delta 5.83-5.68(\mathrm{~m}, 1 \mathrm{H})$, $5.37-5.27(\mathrm{~m}, 2 \mathrm{H}), 4.44-4.33(\mathrm{~m}, 4 \mathrm{H}), 2.78(\mathrm{dd}, \mathrm{J}=22.70,7.34 \mathrm{~Hz}, 2 \mathrm{H}) ;{ }^{13} \mathrm{C}-\mathrm{NMR}$ $\left(100 \mathrm{MHz}, \mathrm{CDCl}_{3}\right) \delta 124.86(\mathrm{~d}, J=11.9 \mathrm{~Hz}), 122.51$ (qd, $\left.J=277.6,7.38 \mathrm{~Hz}\right), 122.04$ (d, $J=15.1 \mathrm{~Hz}), 62.27$ (qd, $J=37.8,5.8 \mathrm{~Hz}), 31.36$ (d, $J=141.1$ ); HRMS berechnet für $\mathrm{C}_{7} \mathrm{H}_{9} \mathrm{O}_{3} \mathrm{~F}_{6} \mathrm{P}_{1}$ : 286.0194, gefunden 286.0191. 


\section{Darstellung von Phosphonat 89}

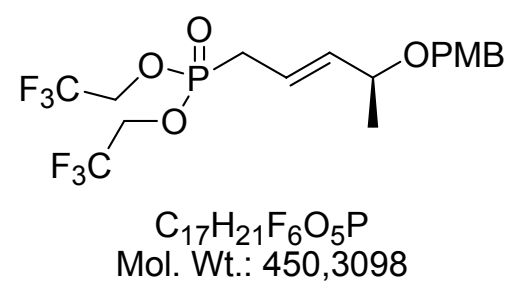

89

Zu einer Lösung von Phosphonat 87 (125 mg, $436.9 \mu \mathrm{mol}$ ) und Alken 88 (84 mg, $436.9 \mu \mathrm{mol})$ in $\mathrm{CH}_{2} \mathrm{Cl}_{2}(2,2 \mathrm{~mL})$ wird Grubbs Katalysator zweiter Generation (16 mg, $21.9 \mu \mathrm{mol}$ ) gegeben und 16 Stunden lang refluxiert. Dann wird das Lösungsmittel im Vakuum entfernt. Nach säulenchromatographischer Reinigung (Hexan/EtOAc $=2: 1$ ) erhält man das gewünschte Produkt $89(120.02 \mathrm{mg}, 266.5 \mu \mathrm{mol}, 61 \%)$ als farbloses Öl: [a] ${ }^{20}{ }_{D}-29.2\left(\mathrm{c} 1.00, \mathrm{CHCl}_{3}\right) ;{ }^{1} \mathrm{H}-\mathrm{NMR}\left(400 \mathrm{MHz}, \mathrm{CDCl}_{3}\right) \delta 7.24(\mathrm{~d}, J=8.9 \mathrm{~Hz}, 2 \mathrm{H})$, $6.9(\mathrm{~d}, J=8.9 \mathrm{~Hz}, 2 \mathrm{H}), 5.74-5.64(\mathrm{~m}, 1 \mathrm{H}), 5.63-5.53(\mathrm{~m}, 1 \mathrm{H}), 4.47(\mathrm{~d}, J=11.3 \mathrm{~Hz}$, $1 \mathrm{H}$ ), $4.43-4.33(\mathrm{~m}, 4 \mathrm{H}), 4.31(\mathrm{~d}, J=11.3 \mathrm{~Hz}, 1 \mathrm{H}$ ), 3.92 (quid, $J=6.5,2.4 \mathrm{~Hz}, 1 \mathrm{H}$ ), $3.80(\mathrm{~s}, 3 \mathrm{H}), 2.79$ (dd, $J=23.4,7.0 \mathrm{~Hz}, 2 \mathrm{H}), 1.25$ (d, $J=6.5 \mathrm{~Hz}, 3 \mathrm{H}) ;{ }^{13} \mathrm{C}-\mathrm{NMR}$ $\left(100 \mathrm{MHz}, \mathrm{CDCl}_{3}\right) \delta 159.14,139.72(\mathrm{~d}, J=15.0 \mathrm{~Hz}), 130.55,129.23,122.54$ (qd, $J=$ 276.8, $6.9 \mathrm{~Hz}$ ), 118.19 (d, $J=12.1 \mathrm{~Hz}), 113.8,74.49$ (d, $J=2.3 \mathrm{~Hz}), 69.70,62.20$ (qdd, $J=37.9,6.2,4.2 \mathrm{~Hz}$ ), 55.27, $29.94(\mathrm{~d}, J=141.1 \mathrm{~Hz}), 21.2(\mathrm{~d}, J=3.1 \mathrm{~Hz}$ ); HRMS berechnet für $\mathrm{C}_{17} \mathrm{H}_{21} \mathrm{O}_{5} \mathrm{~F}_{6} \mathrm{P}_{1}: 450.1031$, gefunden 450.1037 .

Bei dem isolierten Produkt handelt es sich um ein E/Z-Gemisch von 20:1. 


\section{Darstellung von Alkohol 99}

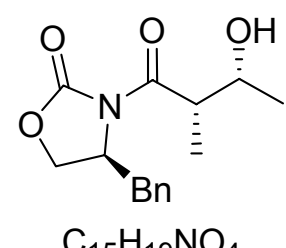

Mol. Wt.: 277,3157

99

Zu Propionyloxazolidinon (98) (1 g, $4.29 \mathrm{mmol})$ gelöst in $\mathrm{CH}_{2} \mathrm{Cl}_{2}(8 \mathrm{~mL})$ wird bei $0{ }^{\circ} \mathrm{C}$ tropfenweise Dibutylbortriflat (1 $\mathrm{M}$ in $\mathrm{CH}_{2} \mathrm{Cl}_{2}, 7.3 \mathrm{~mL}, 7.29 \mathrm{mmol}$ ) und $\mathrm{Et}_{3} \mathrm{~N}(0.79 \mathrm{~mL}$, $5.66 \mathrm{mmol}$ ) gegeben. Dann wird das Reaktionsgemisch auf $-78{ }^{\circ} \mathrm{C}$ abgekühlt und mit Ethanal $(0.48 \mathrm{~mL}, 8.58 \mathrm{mmol})$ versetzt. Nach 20 Minuten wird auf $0{ }^{\circ} \mathrm{C}$ erwärmt und dann eine weitere Stunde gerührt bevor man die Reaktion mit pH 7-Phosphatpuffer $(4.8 \mathrm{~mL})$ und Methanol $(15 \mathrm{~mL})$ quencht. Anschließend gibt man noch ein Gemisch aus Methanol und wässriger $30 \%$ iger $\mathrm{H}_{2} \mathrm{O}_{2}$-Lösung im Verhältnis 2:1 (14.4 mL) hinzu, wobei die Temperatur unter $10^{\circ} \mathrm{C}$ gehalten wird. Nach einer weiteren Stunde wird das $\mathrm{CH}_{2} \mathrm{Cl}_{2}$ im Vakuum entfernt. Anschließend extrahiert man mit MTBE und wäscht die gesammelten organischen Phasen mit gesättigter, wässriger $\mathrm{NaHCO}_{3}$ und $\mathrm{NaCl}$-Lösung und trocknet sie mit $\mathrm{MgSO}_{4}$. Dann filtriert man den Feststoff ab und entfernt das Lösungsmittel im Vakuum. Nach säulenchromatographischer Reinigung (Hexan/EtOAc $=2: 1)$ erhält man das gewünschte Produkt 99 (0.65 g, $2.36 \mathrm{mmol}, 55 \%$ ) als farbloses Öl: $[\alpha]^{20}{ }_{\mathrm{D}}+38$ (c 1.00, $\mathrm{CHCl}_{3}$ ) HRMS berechnet für $\mathrm{C}_{15} \mathrm{H}_{19} \mathrm{~N}_{1} \mathrm{O}_{4}$ : 277.1314, gefunden 277.1316. Das Produkt wird sofort in die nächste Stufe eingesetzt. 


\section{Darstellung von $\beta$-Ketoimid 100}<smiles>CC(=O)[C@H](C)C(=O)N1C(=O)OCC1Br</smiles>

$\mathrm{C}_{15} \mathrm{H}_{17} \mathrm{NO}_{4}$

Mol. Wt.: 275,2998

100

Das Aldolprodukt 99 (0.47 g, $1.71 \mathrm{mmol})$ wird in $\mathrm{CH}_{2} \mathrm{Cl}_{2}(8 \mathrm{~mL})$ und DMSO $(8 \mathrm{~mL})$ gelöst. Bei einer Temperatur von $5{ }^{\circ} \mathrm{C}$ wird $\mathrm{Et}_{3} \mathrm{~N}(0.71 \mathrm{~mL}, 5.13 \mathrm{mmol})$ zugegeben. Dann wird auf $0{ }^{\circ} \mathrm{C}$ abgekühlt und $\mathrm{SO}_{3}{ }^{*} \mathrm{Py}(0.82 \mathrm{~g}, 5.13 \mathrm{mmol})$ gelöst in DMSO $(8 \mathrm{~mL})$ addiert. Nach drei Stunden wird das Reaktionsgemisch auf MTBE $(50 \mathrm{~mL})$ gegeben. Die organische Phase wird mit $1 \mathrm{M} \mathrm{NaHSO}_{4}$-Lösung, gesättigter, wässriger $\mathrm{NaHCO}_{3}$ - und $\mathrm{NaCl}$-Lösung gewaschen und dann mit $\mathrm{MgSO}_{4}$ getrocknet. Dann filtriert man den Feststoff ab und entfernt das Lösungsmittel im Vakuum. Nach säulenchromatographischer Reinigung (Hexan/EtOAc $=5: 1)$ erhält man das gewünschte Produkt $100(0.40 \mathrm{~g}, 1.45 \mathrm{mmol}, 85 \%)$ als farbloses Öl: $[\alpha]^{20}{ }_{D}+47$ (c $\left.1.00, \mathrm{CHCl}_{3}\right) ;{ }^{1} \mathrm{H}-\mathrm{NMR}\left(400 \mathrm{MHz}, \mathrm{CDCl}_{3}\right) \delta 7.37-7.23(\mathrm{~m}, 5 \mathrm{H}), 4.72-4.64(\mathrm{~m}$, $1 \mathrm{H}), 4.54(\mathrm{q}, J=7.4 \mathrm{~Hz}, 1 \mathrm{H}), 4.23-4.16(\mathrm{~m}, 2 \mathrm{H}), 3.44(\mathrm{dd}, J=13.5,3.4 \mathrm{~Hz}, 1 \mathrm{H})$, 2.77 (dd, $J=13.5,10.1 \mathrm{~Hz}, 1 \mathrm{H}), 2.34(\mathrm{~s}, 3 \mathrm{H}), 1.43(\mathrm{~d}, J=7.4 \mathrm{~Hz}, 3 \mathrm{H}) ;{ }^{13} \mathrm{C}-\mathrm{NMR}$ $\left(100 \mathrm{MHz} \mathrm{CDCl}_{3}\right) \delta 205.22,169.95,135.72,135.44,129.59,128.94,127.21,66.51$, 55.48, 53.09, 37.51, 28.42, 12.52; HRMS berechnet für $\mathrm{C}_{15} \mathrm{H}_{17} \mathrm{~N}_{1} \mathrm{O}_{4}$ : 275.1158, gefunden 275.1155 . 


\section{Darstellung von Lacton 94}

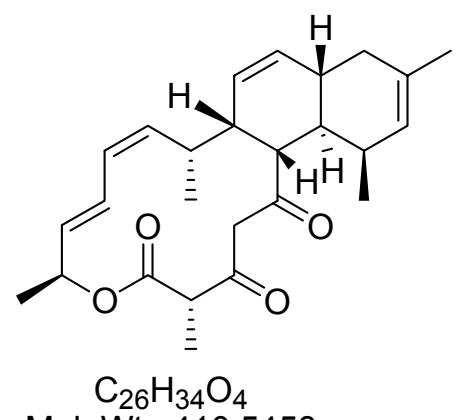

Mol. Wt.: 410,5458

94

1) Zu einer Suspension von Samarium $(70 \mathrm{mg}, 0.46 \mathrm{mmol})$ in THF $(0.44 \mathrm{~mL})$ wird bei Raumtemperatur eine Lösung von Diiodethan (124 mg, $0.44 \mathrm{mmol}$ ) in THF $(0.44 \mathrm{~mL})$ gegeben. Dabei muss unbedingt darauf geachtet werden, dass entgastes THF verwendet wird. Nach einer Stunde hat sich eine tiefblaue Samariumdiiodid-Lösung gebildet, die zu einer Suspension von Chlorotonil $A$ (3) $(50 \mathrm{mg}, 0.1043 \mathrm{mmol})$ in THF/Methanol $(0.21 \mathrm{~mL} / 0.1 \mathrm{~mL})$ bei $-78{ }^{\circ} \mathrm{C}$ gegeben wird. Es wird eine Stunde bei $-78{ }^{\circ} \mathrm{C}$ gerührt und dann mit EtOAc (6 mL) und $0.1 \mathrm{M} \mathrm{HCl}(6 \mathrm{~mL})$ gequencht. Die wässrige Phase wird mit EtOAc extrahiert. Die gesammelten organischen Phasen werden mit gesättigter, wässriger $\mathrm{NaHCO}_{3}$-Lösung gewaschen und mit $\mathrm{MgSO}_{4}$ getrocknet. Der Feststoff wird abfiltriert und das Lösungsmittel im Vakuum entfernt. Nach säulenchromatographischer Reinigung (Hexan/EtOAc $=5: 1)$ erhält man das gewünschte Produkt 94 (33.40 mg, $81.4 \mu \mathrm{mol}, 78 \%)$ als farblosen Feststoff: $[\alpha]^{20}{ }_{D}+105.22\left(\mathrm{c} 1.00, \mathrm{CHCl}_{3}\right) ;{ }^{1} \mathrm{H}-\mathrm{NMR}\left(400 \mathrm{MHz}, \mathrm{CDCl}_{3}\right) \delta 16.14(\mathrm{~s}, 1 \mathrm{H})$, 6.20 (dd, $J=14.0,11.2 \mathrm{~Hz}, 1 \mathrm{H}), 5.81(\mathrm{t}, J=10.4 \mathrm{~Hz}, 1 \mathrm{H}), 5.70(\mathrm{~d}, J=10.4 \mathrm{~Hz}$, $1 \mathrm{H}), 5.69-5.61(\mathrm{~m}, 1 \mathrm{H}), 5.50(\mathrm{dq}, J=10.0,2.1 \mathrm{~Hz}, 1 \mathrm{H}), 5.48-5.32(\mathrm{~m}, 4 \mathrm{H})$, 4.59 (q, $J=6.6 \mathrm{~Hz}, 1 \mathrm{H}$ ), 3.39 (dd, $J=11.8,7.1 \mathrm{~Hz}, 1 \mathrm{H}), 2.92$ (br s, 1H), 2.70 (br s, 1H), $2.45-2.35(\mathrm{~m}, 1 \mathrm{H}), 2.14-2.05(\mathrm{~m}, 1 \mathrm{H}), 2.05-2.00(\mathrm{~m}, 2 \mathrm{H}), 1.77$ (s, 3H), $1.76-1.65(\mathrm{~m}, 1 \mathrm{H}), 1.37(\mathrm{~d}, J=6.6 \mathrm{~Hz}, 3 \mathrm{H}), 1.00(\mathrm{~d}, J=7.0 \mathrm{~Hz}, 3 \mathrm{H})$, $0.75(\mathrm{~d}, J=6.5 \mathrm{~Hz}, 3 \mathrm{H}), 0.62(\mathrm{~d}, J=7.1 \mathrm{~Hz}, 3 \mathrm{H}) ;{ }^{13} \mathrm{C}-\mathrm{NMR}\left(100 \mathrm{MHz}, \mathrm{CDCl}_{3}\right)$ $\delta$ 194.09, 180.03, 175.03, 139.51, 133.51, 130.81, 130.25, 130.24, 125.30, $124.59,124.22,107.67,69.41,48.25,45.25,41.45,38.43,36.2933 .76,30.89$, 
$30.66,23.89,20.47,16.47,15.50,14.47$; HRMS berechnet für $\mathrm{C}_{26} \mathrm{H}_{35} \mathrm{O}_{4}$ $\left(\mathrm{C}_{26} \mathrm{H}_{34} \mathrm{O}_{4}\right.$ plus $\left.\mathrm{H}\right):$ 411.2535, gefunden 411.2521.

2) PMB-Ether 107 (und die Verunreinigung Ethyl-2-methylacetoacetat (109)) wird in Toluol $(10 \mathrm{~mL})$ gelöst und mit zwei Tropfen $\mathrm{BF}_{3}{ }^{*} \mathrm{OEt}_{2}$ versetzt. Nach 15 Minuten wird die Reaktionsmischung mit gesättigter, wässriger $\mathrm{NaHCO}_{3}$-Lösung $(0.5 \mathrm{~mL})$ gequencht. Die Phasen werden getrennt und die wässrige wird mit EtOAc extrahiert. Die gesammelten organischen Phasen werden mit gesättigter, wässriger $\mathrm{NaCl}$-Lösung gewaschen und über $\mathrm{MgSO}_{4}$ getrocknet. Der Feststoff wird abfiltriert und das Lösungsmittel im Vakuum entfernt. Nach säulenchromatographischer Reinigung (Hexan/EtOAc $=5: 1$ ) erhält man das gewünschte Produkt 94 (19.3 mg, $0.05 \mathrm{mmol}$, 47\% über zwei Stufen) als farblosen Feststoff. 


\section{Darstellung von Chlorotonil A (3)}

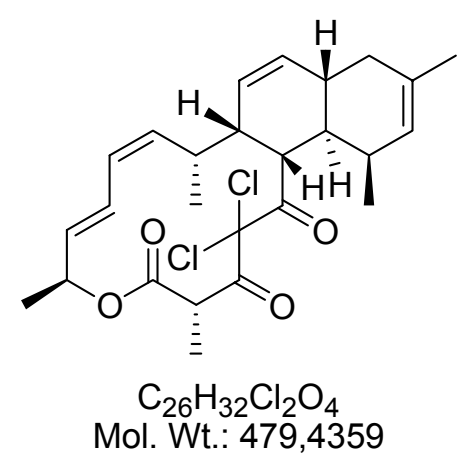

(3)

Lacton 94 (10 mg, $24.4 \mu \mathrm{mol})$ wird in $\mathrm{CH}_{2} \mathrm{Cl}_{2}$ gelöst $(2 \mathrm{~mL})$ und mit 2,6-Lutidin $(2 \mu \mathrm{L}$, $13.57 \mu \mathrm{mol})$ und $\mathrm{N}$-Chlorsuccinimid $(6.83 \mathrm{mg}, 51.2 \mu \mathrm{mol})$ bei Raumtemperatur versetzt. Nach zwei Stunden wird das Reaktionsgemisch mit EtOAc verdünnt und mit gesättigter, wässriger $\mathrm{NaCl}$-Lösung gewaschen. Die organische Phase wird mit $\mathrm{MgSO}_{4}$ getrocknet. Dann filtriert man den Feststoff ab und entfernt das Lösungsmittel im Vakuum. Nach säulenchromatographischer Reinigung (Hexan:EtOAc = 5:1) und Umkristallisation aus Methanol erhält man das gewünschte Produkt Chlorotonil A (3) (7.6 mg,15.8 $\mu \mathrm{mol}, 65 \%$ ) als farblosen Feststoff: $[\alpha]^{20}-180.9$ (c 1.00, $\mathrm{CHCl}_{3}$ ); ${ }^{1} \mathrm{H}-\mathrm{NMR}\left(400 \mathrm{MHz}, \mathrm{CDCl}_{3}\right) \delta 6.08(\mathrm{t}, J=12.8 \mathrm{~Hz}, 1 \mathrm{H}), 5.91(\mathrm{t}, J=10.4 \mathrm{~Hz}, 1 \mathrm{H}), 5.77$ $(\mathrm{d}, J=10.4 \mathrm{~Hz}, 1 \mathrm{H}), 5.70-5.60(\mathrm{~m}, 1 \mathrm{H}), 5.59-5.51(\mathrm{~m}, 2 \mathrm{H}), 5.45-5.39(\mathrm{~m}, 1 \mathrm{H})$, $5.33(\mathrm{t}, J=9.4 \mathrm{~Hz}, 1 \mathrm{H}), 4.57(\mathrm{q}, J=7.1 \mathrm{~Hz}, 1 \mathrm{H}), 3.81(\mathrm{dd}, J=11.8,6.9 \mathrm{~Hz}, 1 \mathrm{H}), 3.05$ (br s, 1H), 2.81 (br s, 1H), 2.39 (br s, 1H), $2.26-2.14(\mathrm{~m}, 2 \mathrm{H}), 2.07$ (d, J = $17.1 \mathrm{~Hz}$, $1 \mathrm{H}), 1.87-1.73(\mathrm{~m}, 1 \mathrm{H}), 1.69(\mathrm{~s}, 3 \mathrm{H}), 1.68(\mathrm{~d}, J=7.1 \mathrm{~Hz}, 3 \mathrm{H}), 1.35(\mathrm{~d}, J=6.8 \mathrm{~Hz}$, $3 \mathrm{H}), 0.98(\mathrm{~d}, J=6.5 \mathrm{~Hz}, 3 \mathrm{H}), 0.87(\mathrm{~d}, J=7.2 \mathrm{~Hz}, 3 \mathrm{H}) ;{ }^{13} \mathrm{C}-\mathrm{NMR}\left(100 \mathrm{MHz}, \mathrm{CDCl}_{3}\right)$ $\delta$ 196.76, 192.00, 167.90, 139.28, 133.10, 132.27, 130.24, 127.98, 125.42, 123.87, 123.50, 81.51, 70.25, 49.62, 47.03, 42.68, 38.29, 36.72, 33.32, 30.28, 30.13, 23.22, 20.92, 17.05, 15.64, 14.77; HRMS berechnet für $\mathrm{C}_{28} \mathrm{H}_{35} \mathrm{O}_{4} \mathrm{~N}_{1} \mathrm{Cl}_{2} \mathrm{Na}_{1}\left(\mathrm{C}_{26} \mathrm{H}_{32} \mathrm{O}_{4} \mathrm{Cl}_{2}\right.$ plus MeCN plus Na): 542.1841, gefunden 542.1848. 


\section{Darstellung von Bromotonil 116}

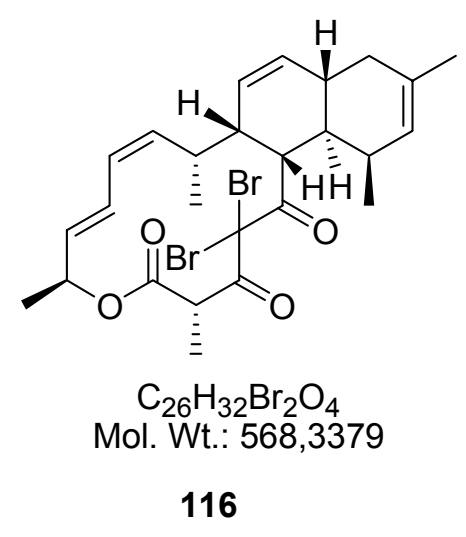

Lacton 94 (10 mg, $24.4 \mu \mathrm{mol})$ wird in $\mathrm{CH}_{2} \mathrm{Cl}_{2}$ gelöst $(2 \mathrm{~mL})$ und mit 2,6-Lutidin $(2 \mu \mathrm{L}$, $13.57 \mu \mathrm{mol})$ und $N$-Bromsuccinimid $(9.11 \mathrm{mg}, 51.2 \mu \mathrm{mol})$ bei Raumtemperatur versetzt. Nach zwei Stunden wird das Reaktionsgemisch mit EtOAc verdünnt und mit gesättigter, wässriger $\mathrm{NaCl}$-Lösung gewaschen. Die organische Phase wird mit $\mathrm{MgSO}_{4}$ getrocknet. Dann filtriert man den Feststoff ab und entfernt das Lösungsmittel im Vakuum. Nach säulenchromatographischer Reinigung (Hexan:EtOAc $=5: 1$ ) und Umkristallisation aus Methanol erhält man das gewünschte Produkt 116 (8.4 mg, $14.9 \mu \mathrm{mol}, 61 \%$ ) als farblosen Feststoff: $[\alpha]^{20}-193.8$ (c 1.00, $\mathrm{CHCl}_{3}$ ); ${ }^{1} \mathrm{H}-\mathrm{NMR}$ $\left(400 \mathrm{MHz}, \mathrm{CDCl}_{3}\right) \delta 6.07(\mathrm{t}, J=14.2 \mathrm{~Hz}, 1 \mathrm{H}), 5.90(\mathrm{t}, J=11.1 \mathrm{~Hz}, 1 \mathrm{H}), 5.77(\mathrm{~d}, J=$ $9.90 \mathrm{~Hz}, 1 \mathrm{H}), 5.67-5.59(\mathrm{~m}, 1 \mathrm{H}), 5.58-5.50(\mathrm{~m}, 2 \mathrm{H}), 5.47-5.40(\mathrm{~m}, 1 \mathrm{H}), 5.33(\mathrm{t}$, $J=9.39 \mathrm{~Hz}, 1 \mathrm{H}), 4.64(\mathrm{q}, J=7.1 \mathrm{~Hz}, 1 \mathrm{H}), 3.82(\mathrm{dd}, J=11.6,6.5 \mathrm{~Hz}, 1 \mathrm{H}), 3.07-2.99$ $(\mathrm{m}, 1 \mathrm{H}), 2.81-2.66(\mathrm{~m}, 1 \mathrm{H}), 2.47-2.34(\mathrm{~m}, 1 \mathrm{H}), 2.28-2.16(\mathrm{~m}, 2 \mathrm{H}), 2.13-2.04$ $(\mathrm{m}, 1 \mathrm{H}), 1.85-1.71(\mathrm{~m}, 1 \mathrm{H}), 1.78(\mathrm{~d}, J=7.1 \mathrm{~Hz}, 3 \mathrm{H}), 1.70(\mathrm{~s}, 3 \mathrm{H}), 1.35(\mathrm{~d}, J=$ $6.8 \mathrm{~Hz}, 3 \mathrm{H}), 0.98(\mathrm{~d}, J=6.5 \mathrm{~Hz}, 3 \mathrm{H}), 0.94(\mathrm{~d}, J=6.8 \mathrm{~Hz}, 3 \mathrm{H}) ;{ }^{13} \mathrm{C}-\mathrm{NMR}(100 \mathrm{MHz}$, $\left.\mathrm{CDCl}_{3}\right) \delta 196.74,191.77,167.92,139.35,133.08,132.27,130.20,128.14,125.40$, $123.76,123.53,71.66,70.28,49.38,47.50,42.46,38.36,36.80,33.37,30.18,29.71$, 23.24, 20.92, 18.02, 15.65, 15.14; HRMS berechnet für $\mathrm{C}_{28} \mathrm{H}_{35} \mathrm{O}_{4} \mathrm{~N}_{1} \mathrm{Br}_{2} \mathrm{Na}_{1}$ $\left(\mathrm{C}_{26} \mathrm{H}_{32} \mathrm{O}_{4} \mathrm{Br}_{2}\right.$ plus MeCN plus $\left.\mathrm{Na}\right): 630.0667$, gefunden 630.0669 . 


\section{Literaturverzeichnis}

[1] A. Limberg, D. Schinzer, Magdburger Wissenschaftsjournal 2000, 1, 23.

[2] M. Dworkin, Microbiol. Rev. 1996, 60, 70.

[3] K. Gerth, R. Müller, Environ. Microbiol. 2005, 7, 874.

[4] K. Gerth, R. Müller, S. Pradella, O. Perlova, S. Beyer, J. Biotechnol. 2003, 106, 233.

[5] Die Struktur wurde im Jahre 2004 bei den 17. Irseeer Naturstofftagen präsentiert.

[6] R. Frank, Biospektrum, 2005, 8. Jahrgang, 474.

[7] K. Takao, R. Munakata, K. Tadano, Chem. Rev. 2005, 105, 4779.

[8] J. Inanaga, K. Hirata, H.Saeki, T. Katsuki, M. Yamaguchi, Bull. Chem. Soc. Jpn. 1979, 52, 1989.

[9] I. Dhimitruka, J. SantaLucia, Org. Lett., 2006, 8, 47.

[10] D. L. Rieger, D. A. Evans, J. Am. Chem. Soc. 1993, 115, 11446.

[11] W. C. Still, C. Gennari, Tetrahedron Lett. 1983, 41, 4405.

[12] G. Wittig, U. Schöllkopf, Ber. 1954, 87, 1318; B. E Maryanoff, A. B. Reitz, Chem. Rev. 1989, 89, 863-927.

[13] D. A. Evans, J. S. Clark, R. Metternich, V. J. Novack, G. S. Sheppard, J. Am. Chem. Soc. 1990, 112, 866.

[14] S. A. Frank, W. R. Roush, J. Org. Chem. 2002, 67, 4316.

[15] E. Kattnig, O. Lepage, A. Fürstner, J. Am. Chem. Soc. 2006, 128, 9194.

[16] C. Morrill, R. H. Grubbs, J. Org. Chem. 2003, 68, 6031.

[17] E. J. Corey, P. L. Fuchs, Tetrahedron Lett. 1972, 36, 3769.

[18] K. P. Chary, M. Quitschalle, A. Burzlaff, C. Kasper, T. Scheper, M. Kalesse, Chem. Eur. J. 2003, 9, 1129.

[19] A. Villalobos, S. J. Danishefsky, J. Org. Chem. 1990, 55, 2776.

[20] S. A. Frank, H. Chen, R. K. Kunz, M. J. Schnaderbeck, W. R. Roush, Organic Letters, 2000, 17, 2691.

[21] N. Miyaura, A. Suzuki, Chem. Rev. 1995, 95, 2457.

[22] J. Uenishi, J.-M. Beau, R. W. Armstrong, Y. Kishi, J. Am. Chem. Soc. 1987, 109, 4756.

[23] J. T. Starr, D. A. Evans, J. Am. Chem. Soc. 2003, 125, 13531.

[24] D. J. Mergott, S. A. Frank, W. R. Roush, Organic Letters 2002, 18, 3157. 
[25] K. Koyama, M. L. Curtin, K. J. Moriarty, W. R. Roush, J. Am. Chem. Soc. 1996, $118,7502$.

[26] D. T. Mao, S. R. Wilson, J. Am. Chem. Soc. 1978, 100, 6289.

[27] M. Kageyama, R. Riva, B. B. Brown, J. S. Warmus, K. J. Moriarty, W. R. Roush, J. Org. Chem. 1991, 56, 1192.

[28] K. Yang, T. K. Shing, J. Org. Chem. 1995, 60, 5785.

[29] J. E. Audia, B. Shearer, J. A. Marshall, J. Org. Chem. 1986, 51, 1730.

[30] A. Padwa, L. S. Beall, T. M. Heidelbaugh, B. Liu, S. M. Sheehan, J. Org. Chem. 2000, 65, 2684.

[31] M. S. Congreve, E.C. Davison, M. A. M. Fuhry, A. N. Payne, R. A. Robinson, S. E. Ward, A. B. Holmes, Synlett 1993, 663.

[32] A. J. Mancuso, D. Swern, Synthesis 1981, 165.

[33] R. Appel, Angew. Chem. Int. Ed. 1975, 14, 801; T. W. Baughman, J. C. Sworen, K. B. Wagener, Tetrahedron, 2004, 60, 10943.

[34] S. R. Chemler, D. S. Coffey, W. R. Roush, Tetrahedron Lett. 1999, 40, 1269.

[35] S. J. Miller, M. D. Ennis, D. A. Evans, J. Org. Chem. 1993, 58, 471.

[36] A. E. Arbuzov, J. Russ. Phys. Chem. Soc. 1906, 38, 687; A. Ianni, S. R. Waldvogel, Synthesis, 2006, 2103; V. K. Aggarwal, J. R. Fulton, C. G. Sheldon, J. de Vicente, J. Am. Chem. Soc., 2003, 125, 6034.

[37] B. M. Brandt, A. B. Smith, Organic Letters 2001, 3, 1685.

[38] Y. Yuh, N. L. Allinger, QCPE 1980, 12, 395.

[39] C. R. Holmquist, E. J. Roskamp, J. Org. Chem. 1989, 54, 3258.

[40] C. Neukom, M. Rosenberger, J. Org. Chem. 1982, 47, 1779.

[41] G. Hahn, G. A. Molander, J. Org. Chem. 1986, 51, 1135.

[42] W.S. Weiner, N. Maslouh, R. V. Hoffman, J. Org. Chem. 2001, 66, 5790.

[43] A. Matlack, J. Org. Chem. 1958, 23, 729.

[44] H. A. Dieck, R. F. Heck, J. Org. Chem. 1975, 40, 1083.

[45] M. Beller, T. H. Riermeier, G. Stark in Transition Metals in Organic Synthesis, Vol. 1, Wiley-VCH, Weinheim, 1998, 208.

[46] T. Jeffery, J. Chem. Soc. Chem. Commun. 1984, 1287.

[47] T. V. Rajan Babu, T. Fukunaya, G. S. Reddy, J. Am. Chem. Soc. 1989, 111, 1759. 


\section{Diels-Alder Produkt 8}

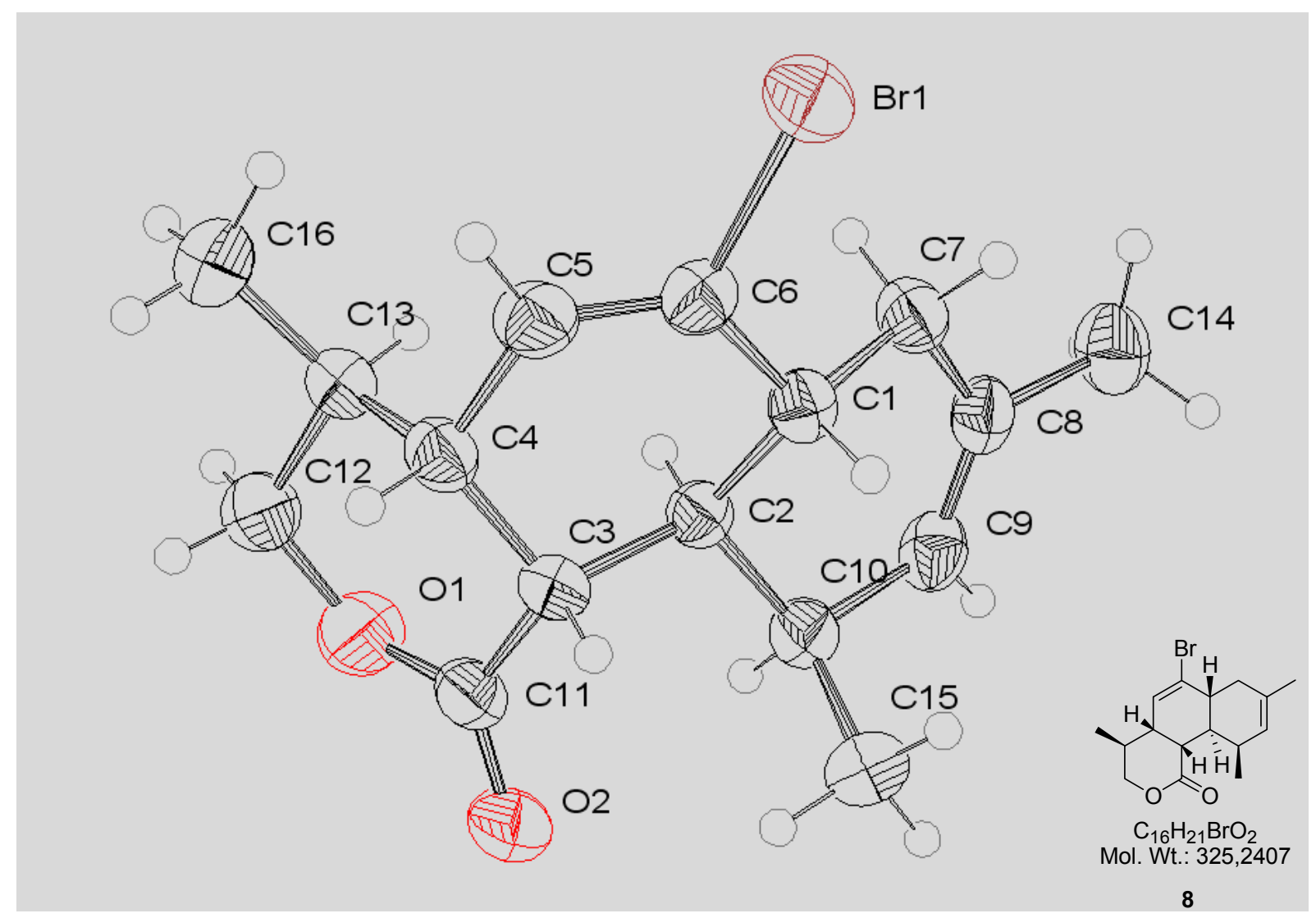

Abbildung 1 ORTEP Zeichnung des Diels-Alder Produktes 8.

\begin{tabular}{|l|l|}
\hline \multicolumn{2}{|c|}{ Crystal Data and structure refinement } \\
\hline Empirical Formula & $\mathrm{C}_{16} \mathrm{H}_{21} \mathrm{O}_{2} \mathrm{Br}$ \\
\hline Formula Weight & 325.24 \\
\hline Temperature $(\mathrm{K})$ & 298 \\
\hline Wavelength $(\mathrm{pm})$ & 71.073 \\
\hline Crystal System & Monoclinic \\
\hline Space group & $\mathrm{P} 21$ \\
\hline Unit cell dimensions $(\mathrm{pm})$ & $\begin{array}{l}\mathrm{a}=970.5(4), \alpha=90^{\circ} \\
\mathrm{b}=589.4(16), \mathrm{B}=102.32(5) \\
\mathrm{C}=1323.6(6), \mathrm{V}=90^{\circ}\end{array}$ \\
\hline Volume $\left(\mathrm{nm}^{3}\right)$ & $0.7397(5)$ \\
\hline Calculated density $\left(\mathrm{g} \mathrm{cm}^{-3}\right)$ & 1.460 \\
\hline F (000) & 336 \\
\hline Crystal size $\left(\mathrm{mm}^{3}\right)$ & $0.40 \times 0.30 \times 0.08$ \\
\hline
\end{tabular}




\begin{tabular}{|l|l|}
\hline అ-range for data collection & $2.15^{\circ}-26.12^{\circ}$ \\
\hline Limiting indices & $-11 \leq \mathrm{h} \leq 11,-6 \leq \mathrm{k} \leq 6,-16 \leq \mathrm{I} \leq 16$ \\
\hline Reflections collected & 10491 \\
\hline Independent reflections & 2800 \\
\hline $\mathrm{R}$ & $\mathrm{R} 1=0.0354, \mathrm{wR} 2=0.0652, \mathrm{~S}=-0.012(12)$ \\
\hline
\end{tabular}

\begin{tabular}{|c|c|c|c|c|}
\hline \multicolumn{5}{|c|}{$\begin{array}{l}\text { Atomic coordinates and equivalent isotropic displacement parameters } U(e q) \text { is } \\
\text { defined as one third of the trace of the orthogonalized } U_{i j} \text { tensor }\end{array}$} \\
\hline Atom & $\mathbf{x}$ & $y$ & $\mathbf{z}$ & $\mathrm{U}(\mathrm{eq})\left[\AA^{2}\right]$ \\
\hline $\mathrm{C} 1$ & $0.7377(4)$ & $-0.3629(7)$ & $0.3059(3)$ & $0.036(1)$ \\
\hline $\mathrm{C} 2$ & $0.7684(4)$ & $-0.4949(7)$ & $0.2123(3)$ & $0.035(1)$ \\
\hline C3 & $0.8468(4)$ & $-0.3439(8)$ & $0.1461(3)$ & $0.039(1)$ \\
\hline $\mathrm{C} 4$ & $0.9861(4)$ & $-0.2504(8)$ & $0.2106(3)$ & $0.040(1)$ \\
\hline $\mathrm{C} 5$ & $0.9683(4)$ & $-0.1582(8)$ & $0.3130(3)$ & $0.045(2)$ \\
\hline C6 & $0.8601(4)$ & $-0,2174(8)$ & $0.3551(3)$ & $0.039(1)$ \\
\hline $\mathrm{C7}$ & $0.6924(4)$ & $-0.5334(7)$ & $0.3799(3)$ & $0.043(1)$ \\
\hline C8 & $0.5862(4)$ & $-0.7010(8)$ & $0.3272(3)$ & $0.043(1)$ \\
\hline C9 & $0.5604(4)$ & $-0.7315(8)$ & $0.2260(3)$ & $0.046(1)$ \\
\hline $\mathrm{C} 10$ & $0.6301(3)$ & $-0.5957(14)$ & $0.1522(2)$ & $0.042(1)$ \\
\hline C11 & $0.8671(4)$ & $-0.4757(8)$ & $0.0519(3)$ & $0.047(1)$ \\
\hline C12 & $1.1184(4)$ & $-0.5188(9)$ & $0.1220(3)$ & $0.058(2)$ \\
\hline C13 & $1.1044(4)$ & $-0.4242(8)$ & $0.2258(3)$ & $0.045(1)$ \\
\hline C14 & $0.5068(5)$ & $-0.8284(10)$ & $0.3959(3)$ & $0.059(1)$ \\
\hline C15 & $0.5247(4)$ & $-0.4340(10)$ & $0.0894(3)$ & $0.059(1)$ \\
\hline C16 & $1.2464(4)$ & $-0.3278(10)$ & $0.2795(4)$ & $0.064(1)$ \\
\hline $\mathrm{Br} 1$ & $0.8606(1)$ & $-0.1043(1)$ & $0.4905(1)$ & $0.053(1)$ \\
\hline 01 & $0.9868(3)$ & $-0.5958(11)$ & $0.0579(2)$ & $0.063(1)$ \\
\hline $\mathrm{O} 2$ & $0.7769(3)$ & $-0.4935(6)$ & $-0.0261(2)$ & $0.062(1)$ \\
\hline
\end{tabular}




\begin{tabular}{|c|c|c|c|}
\hline \multicolumn{4}{|c|}{ Bond lengths [pm] } \\
\hline $\mathrm{C} 1-\mathrm{C} 6$ & $149.7(6)$ & C7 - C8 & $149.0(5)$ \\
\hline $\mathrm{C} 1-\mathrm{C} 7$ & $153.2(5)$ & $\mathrm{C} 8-\mathrm{C} 9$ & $132.2(5)$ \\
\hline $\mathrm{C} 1-\mathrm{C} 2$ & $154.5(5)$ & $\mathrm{C} 8-\mathrm{C} 14$ & $151.1(6)$ \\
\hline $\mathrm{C} 2-\mathrm{C} 10$ & $152.7(6)$ & C9-C10 & $152.8(7)$ \\
\hline $\mathrm{C} 2-\mathrm{C} 3$ & $155.7(5)$ & C10-C15 & $151.0(7)$ \\
\hline C3-C11 & $151.7(6)$ & $C(11-02$ & $120.8(5)$ \\
\hline $\mathrm{C} 3-\mathrm{C} 4$ & $153.9(5)$ & C11-O1 & $134.8(6)$ \\
\hline $\mathrm{C} 4-\mathrm{C} 5$ & $150.4(5)$ & $\mathrm{C} 12-\mathrm{O} 1$ & $144.7(5)$ \\
\hline $\mathrm{C} 4-\mathrm{C} 13$ & $151.9(6)$ & $\mathrm{C} 12-\mathrm{C} 13$ & $151.5(5)$ \\
\hline C5- C6 & $133.7(5)$ & C13-C16 & $152.0(6)$ \\
\hline $\mathrm{C} 6-\mathrm{Br} 1$ & $191.2(4)$ & & \\
\hline
\end{tabular}

\begin{tabular}{|c|c|c|c|}
\hline \multicolumn{4}{|c|}{ Bond angles $\left[{ }^{\circ}\right]$} \\
\hline $\mathrm{C} 6-\mathrm{C} 1-\mathrm{C} 7$ & $114.0(3)$ & $\mathrm{C} 8-\mathrm{C} 7-\mathrm{C} 1$ & $113.6(3)$ \\
\hline $\mathrm{C} 6-\mathrm{C} 1-\mathrm{C} 2$ & $111.5(3)$ & $\mathrm{C} 9-\mathrm{C} 8-\mathrm{C} 7$ & $121.8(4)$ \\
\hline $\mathrm{C} 7-\mathrm{C} 1-\mathrm{C} 2$ & $108.2(3)$ & $\mathrm{C} 9-\mathrm{C} 8-\mathrm{C} 14$ & $122.2(4)$ \\
\hline $\mathrm{C} 10-\mathrm{C} 2-\mathrm{C} 1$ & $108.4(3)$ & C7 - C8 - C14 & $116.0(4)$ \\
\hline $\mathrm{C} 10-\mathrm{C} 2-\mathrm{C} 3$ & $114.2(3)$ & $\mathrm{C} 8-\mathrm{C} 9-\mathrm{C} 10$ & $124.3(4)$ \\
\hline $\mathrm{C} 1-\mathrm{C} 2-\mathrm{C} 3$ & $111.4(3)$ & $\mathrm{C} 15-\mathrm{C} 10-\mathrm{C} 2$ & $117.2(6)$ \\
\hline $\mathrm{C} 11-\mathrm{C} 3-\mathrm{C} 4$ & $112.7(3)$ & C15-C10-C9 & $110.0(3)$ \\
\hline $\mathrm{C} 11-\mathrm{C} 3-\mathrm{C} 2$ & $109.5(3)$ & C2 $-\mathrm{C} 10-\mathrm{C} 9$ & $109.3(3)$ \\
\hline C4- C3-C2 & $111.7(3)$ & $\mathrm{O} 2-\mathrm{C} 11-\mathrm{O} 1$ & $117.6(4)$ \\
\hline $\mathrm{C} 5-\mathrm{C} 4-\mathrm{C} 13$ & $110.7(3)$ & $\mathrm{O} 2-\mathrm{C} 11-\mathrm{C} 3$ & $123.3(4)$ \\
\hline $\mathrm{C} 5-\mathrm{C} 4-\mathrm{C} 3$ & $111.9(3)$ & $\mathrm{O} 1-\mathrm{C} 11-\mathrm{C} 3$ & $118.8(3)$ \\
\hline $\mathrm{C} 13-\mathrm{C} 4-\mathrm{C} 3$ & $112.4(4)$ & $\mathrm{O} 1-\mathrm{C} 12-\mathrm{C} 13$ & $114.1(3)$ \\
\hline $\mathrm{C} 6-\mathrm{C} 5-\mathrm{C} 4$ & $122.2(4)$ & $\mathrm{C} 12-\mathrm{C} 13-\mathrm{C} 4$ & 109.9 (3) \\
\hline $\mathrm{C} 5-\mathrm{C} 6-\mathrm{C} 1$ & $126.2(3)$ & $\mathrm{C} 12-\mathrm{C} 13-\mathrm{C} 16$ & $108.3(3)$ \\
\hline $\mathrm{C} 5-\mathrm{C} 6-\mathrm{Br} 1$ & $117.0(3)$ & $\mathrm{C} 4-\mathrm{C} 13-\mathrm{C} 16$ & $113.2(4)$ \\
\hline $\mathrm{C} 1-\mathrm{C} 6-\mathrm{Br} 1$ & $116.8(3)$ & $\mathrm{C} 11-\mathrm{O} 1-\mathrm{C} 12$ & $120.7(5)$ \\
\hline
\end{tabular}




\section{Diels-Alder Produkt 46}

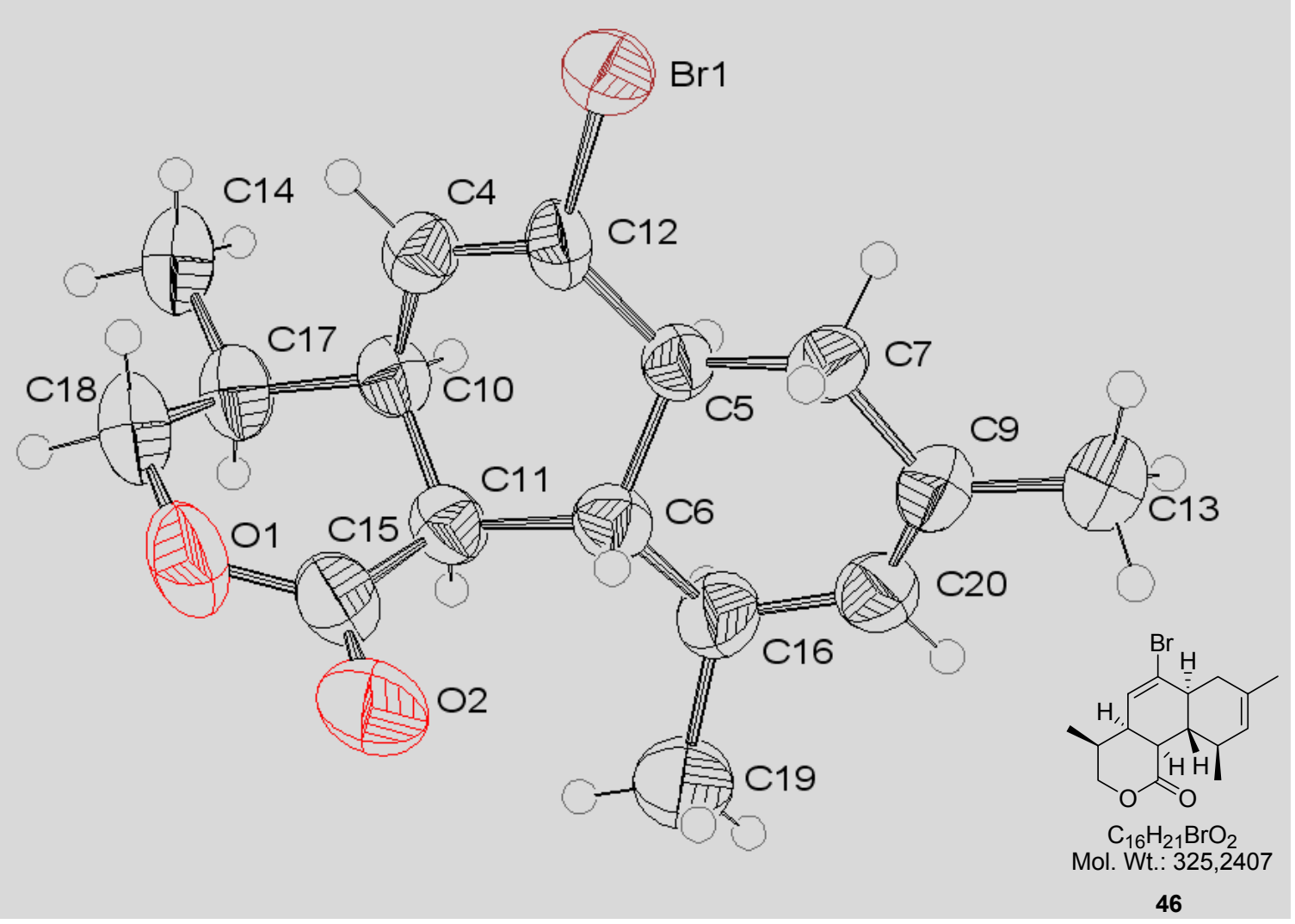

Abbildung 2 ORTEP Zeichnung des Diels-Alder Produktes 46.

\begin{tabular}{|l|l|}
\hline \multicolumn{2}{|c|}{ Crystal Data and structure refinement } \\
\hline Empirical Formula & $\mathrm{C}_{16} \mathrm{H}_{21} \mathrm{O}_{2} \mathrm{Br}$ \\
\hline Formula Weight & 325.24 \\
\hline Temperature $(\mathrm{K})$ & 298 \\
\hline Wavelength $(\mathrm{pm})$ & 71.073 \\
\hline Crystal System & Orthorhombic \\
\hline Space group & $\mathrm{P} 212121$ \\
\hline Unit cell dimensions $(\mathrm{pm})$ & $\begin{array}{l}\mathrm{a}=556.71(18), \alpha=90^{\circ} \\
\mathrm{b}=1262.0(9), \mathrm{B}=90^{\circ} \\
\mathrm{C}=2148.0(5), \mathrm{Y}=90^{\circ}\end{array}$ \\
\hline Volume $\left(\mathrm{nm}^{3}\right)$ & $1.5091(12)$ \\
\hline Calculated density $\left(\mathrm{g} \mathrm{cm}^{-3}\right)$ & 1.431 \\
\hline F (000) & 672 \\
\hline Crystal size $\left(\mathrm{mm}^{3}\right)$ & $0.20 \times 0.10 \times 0.80$ \\
\hline O-range for data collection & $2.49^{\circ}-26.17^{\circ}$ \\
\hline
\end{tabular}




\begin{tabular}{|l|l|}
\hline Limiting indices & $-6 \leq \mathrm{h} \leq 6,-15 \leq \mathrm{k} \leq 15,-26 \leq \mathrm{I} \leq 26$ \\
\hline Reflections collected & 18614 \\
\hline Independent reflections & 2952 \\
\hline $\mathrm{R}$ & $\mathrm{R} 1=0.0533, \mathrm{wR} 2=0.1187, \mathrm{~S}=-0.02(3)$ \\
\hline
\end{tabular}

\begin{tabular}{|l|l|l|l|l|}
\hline \multicolumn{5}{|c|}{$\begin{array}{l}\text { Atomic coordinates and equivalent isotropic displacement parameters U(eq) is } \\
\text { defined as one third of the trace of the orthogonalized U }{ }_{\text {ij }} \text { tensor }\end{array}$} \\
\hline \multicolumn{1}{|c|}{ Atom } & \multicolumn{1}{|c|}{$\mathbf{x}$} & \multicolumn{1}{c|}{$\mathbf{y}$} & \multicolumn{1}{c|}{$\mathbf{z}$} & $\mathbf{U}(\mathbf{e q})\left[\AA^{2}\right]$ \\
\hline C1 & $0.8726(14)$ & $-0.1890(7)$ & $0.8339(3)$ & $0.050(1)$ \\
\hline C2 & $0.9630(15)$ & $-0.0997(6)$ & $0.9333(3)$ & $0.045(2)$ \\
\hline C3 & $0.9439(14)$ & $0.081(6)$ & $0.9025(4)$ & $0.050(2)$ \\
\hline C4 & $11.462(15)$ & $-0.0937(6)$ & $0.9854(4)$ & $0.053(2)$ \\
\hline C5 & $10.914(17)$ & $0.0019(7)$ & $1.0288(4)$ & $0.055(2)$ \\
\hline C6 & $0.6968(15)$ & $-0,1013(6)$ & $0.8226(3)$ & $0.050(2)$ \\
\hline C7 & $0.8147(15)$ & $0.0048(6)$ & $0.8381(3)$ & $0.053(2)$ \\
\hline C8 & $1.0045(14)$ & $-0.1875(7)$ & $0.8867(3)$ & $0.050(2)$ \\
\hline C9 & $1.2280(20)$ & $-0.0004(7)$ & $1.0889(4)$ & $0.072(2)$ \\
\hline C10 & $0.4589(16)$ & $-0.1972(10)$ & $0.7370(4)$ & $0.0472(2)$ \\
\hline C11 & $0.9780(20)$ & $0.0432(8)$ & $0.7871(4)$ & $0.0065(2)$ \\
\hline C12 & $0.8320(20)$ & $0.0852(7)$ & $0.9487(4)$ & $0.0068(3)$ \\
\hline C13 & $0.5999(17)$ & $-0.0976(9)$ & $0.7566(4)$ & $0.0063(2)$ \\
\hline C14 & $0.8012(19)$ & $-0.0813(9)$ & $0.7119(4)$ & $0.071(3)$ \\
\hline C15 & $0.8610(30)$ & $0.2010(5)$ & $0.9273(5)$ & $0.113(5)$ \\
\hline C16 & $0.9429(18)$ & $0.0 .735(7)$ & $1.0109(4)$ & $0.060(2)$ \\
\hline Br1 & $1.2278(2)$ & $-0.2973(1)$ & $0.9030(1)$ & $0.066(1)$ \\
\hline O1 & $0.9565(15)$ & $0.0084(7)$ & $0.7285(3)$ & $0.084(2)$ \\
\hline O2 & $1.1297(17)$ & $0.1113(7)$ & $0.7951(4)$ & $0.106(3)$ \\
\hline
\end{tabular}




\begin{tabular}{|c|c|c|c|}
\hline \multicolumn{4}{|c|}{ Bond lengths [pm] } \\
\hline $\mathrm{Br} 1-\mathrm{C} 8$ & $189.4(9)$ & $\mathrm{O} 2-\mathrm{C} 11$ & $121.8(12)$ \\
\hline O1-C11 & $133.8(12)$ & C5 - C16 & $131.8(12)$ \\
\hline O1-C14 & $146.8(13)$ & $\mathrm{C} 5-\mathrm{C} 9$ & $149.8(12)$ \\
\hline $\mathrm{C} 1-\mathrm{C} 8$ & $135.0(10)$ & $\mathrm{C} 6-\mathrm{C} 13$ & $151.6(11)$ \\
\hline $\mathrm{C} 1-\mathrm{C} 6$ & $149.8(11)$ & C6-C7 & $152.8(11)$ \\
\hline $\mathrm{C} 2-\mathrm{C} 8$ & $151.2(10)$ & $\mathrm{C} 7-\mathrm{C} 11$ & $150.4(13)$ \\
\hline $\mathrm{C} 2-\mathrm{C} 4$ & $151.5(11)$ & $\mathrm{C} 10-\mathrm{C} 13$ & $154.0(14)$ \\
\hline $\mathrm{C} 2-\mathrm{C} 3$ & $151.7(10)$ & C12-C16 & $147.9(13)$ \\
\hline $\mathrm{C} 3-\mathrm{C} 12$ & $152.2(11)$ & C12-C15 & $154.0(14)$ \\
\hline $\mathrm{C} 3-\mathrm{C} 7$ & $156.0(11)$ & C13-C14 & $149.1(13)$ \\
\hline $\mathrm{C} 4-\mathrm{C} 5$ & $151.8(10)$ & & \\
\hline
\end{tabular}

\begin{tabular}{|c|c|c|c|}
\hline \multicolumn{4}{|c|}{ Bond angles $\left[{ }^{\circ}\right]$} \\
\hline $\mathrm{C} 11-\mathrm{O} 1-\mathrm{C} 14$ & $122.3(8)$ & $\mathrm{C} 15-\mathrm{C} 7-\mathrm{C} 3$ & $111.1(7)$ \\
\hline $\mathrm{C} 8-\mathrm{C} 1-\mathrm{C} 6$ & $118.8(7)$ & $\mathrm{C} 6-\mathrm{C} 7-\mathrm{C} 3$ & $114.5(6)$ \\
\hline $\mathrm{C} 8-\mathrm{C} 2-\mathrm{C} 4$ & $115.0(7)$ & $\mathrm{C} 1-\mathrm{C} 8-\mathrm{C} 2$ & $118.9(8)$ \\
\hline $\mathrm{C} 8-\mathrm{C} 2-\mathrm{C} 3$ & $112.3(6)$ & $\mathrm{C} 1-\mathrm{C} 8-\mathrm{Br} 1$ & $120.1(6)$ \\
\hline $\mathrm{C} 4-\mathrm{C} 2-\mathrm{C} 3$ & $108.9(7)$ & $\mathrm{C} 2-\mathrm{C} 8-\mathrm{Br} 1$ & $120.9(5)$ \\
\hline $\mathrm{C} 2-\mathrm{C} 3-\mathrm{C} 12$ & $108.5(7)$ & $\mathrm{O} 2-\mathrm{C} 11-\mathrm{O} 1$ & $115.3(10)$ \\
\hline $\mathrm{C} 2-\mathrm{C} 3-\mathrm{C} 7$ & $113.3(6)$ & $\mathrm{O} 2-\mathrm{C} 11-\mathrm{C} 7$ & $122.9(9)$ \\
\hline $\mathrm{C} 12-\mathrm{C} 3-\mathrm{C} 7$ & $114.0(7)$ & $\mathrm{O} 1-\mathrm{C} 11-\mathrm{C} 7$ & $121.7(9)$ \\
\hline $\mathrm{C} 2-\mathrm{C} 4-\mathrm{C} 5$ & $110.9(7)$ & $\mathrm{C} 16-\mathrm{C} 12-\mathrm{C} 3$ & $110.7(8)$ \\
\hline $\mathrm{C} 16-\mathrm{C} 5-\mathrm{C} 9$ & $124.1(8)$ & $\mathrm{C} 16-\mathrm{C} 12-\mathrm{C} 15$ & $108.7(8)$ \\
\hline $\mathrm{C} 16-\mathrm{C} 5-\mathrm{C} 4$ & $119.8(7)$ & $\mathrm{C} 2-\mathrm{C} 12-\mathrm{C} 15$ & $111.7(8)$ \\
\hline $\mathrm{C} 9-\mathrm{C} 5-\mathrm{C} 4$ & $116.0(8)$ & $\mathrm{C} 14-\mathrm{C} 13-\mathrm{C} 6$ & $109.8(8)$ \\
\hline $\mathrm{C} 1-\mathrm{C} 6-\mathrm{C} 13$ & $114.0(7)$ & $\mathrm{C} 14-\mathrm{C} 13-\mathrm{C} 10$ & $108.6(8)$ \\
\hline $\mathrm{C} 1-\mathrm{C} 6-\mathrm{C} 7$ & $109.4(7)$ & $\mathrm{C} 6-\mathrm{C} 13-\mathrm{C} 10$ & $114.4(8)$ \\
\hline $\mathrm{C} 13-\mathrm{C} 6-\mathrm{C} 7$ & $109.3(7)$ & $\mathrm{O} 1-\mathrm{C} 14-\mathrm{C} 13$ & $113.1(8)$ \\
\hline $\mathrm{C} 11-\mathrm{C} 7-\mathrm{C} 6$ & $112.5(7)$ & $\mathrm{C} 5-\mathrm{C} 16-\mathrm{C} 12$ & $126.7(8)$ \\
\hline
\end{tabular}




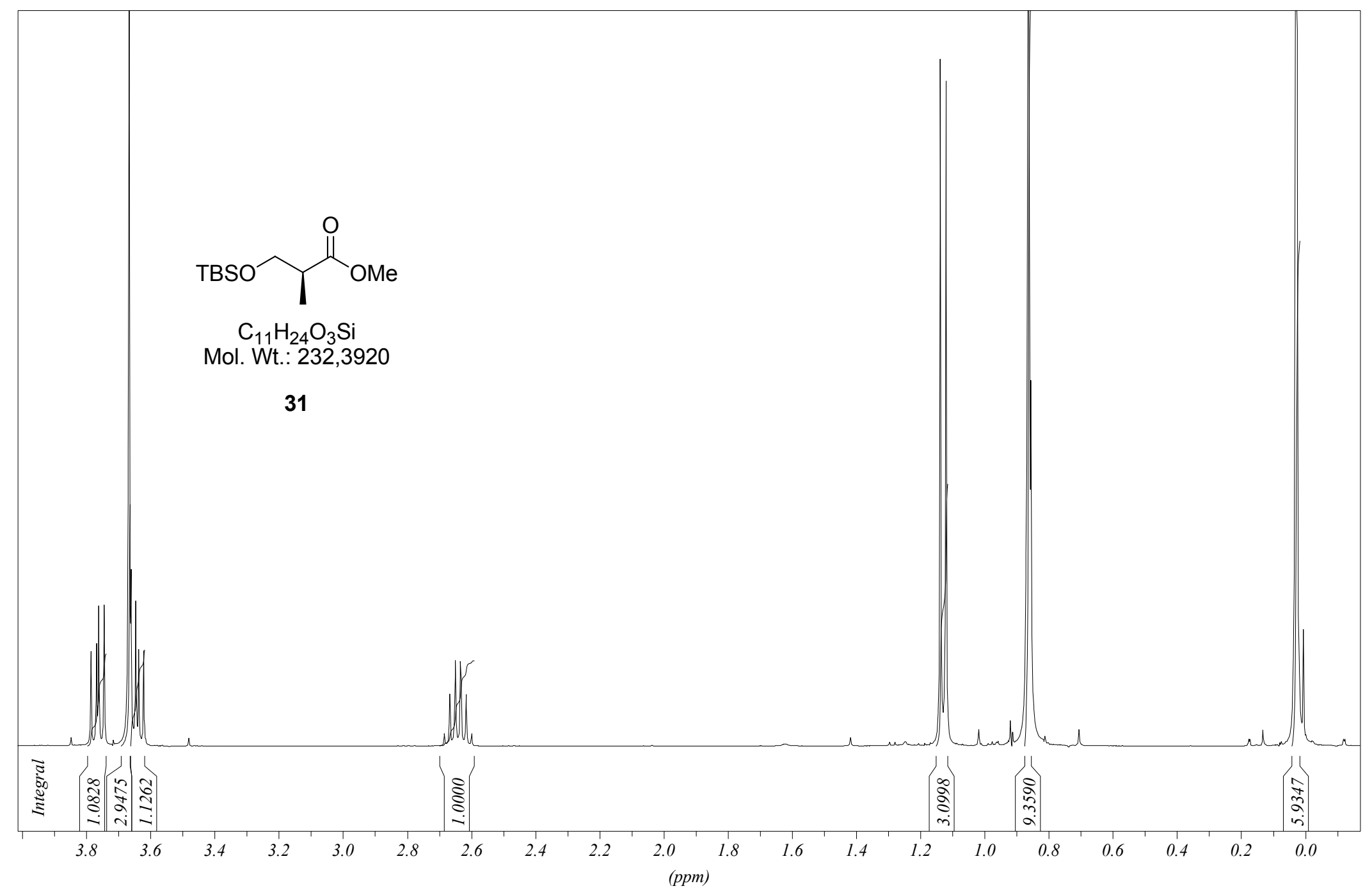




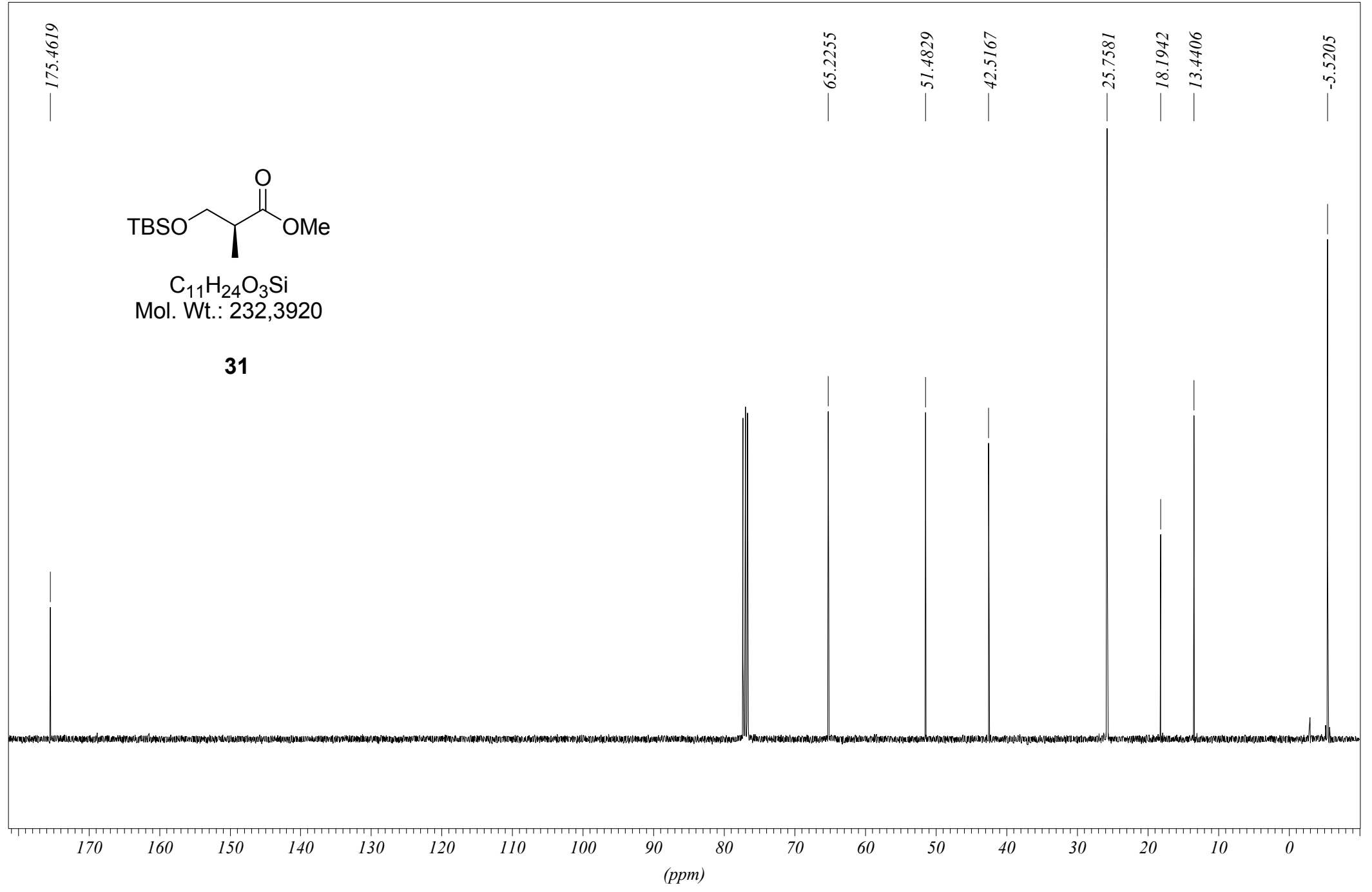




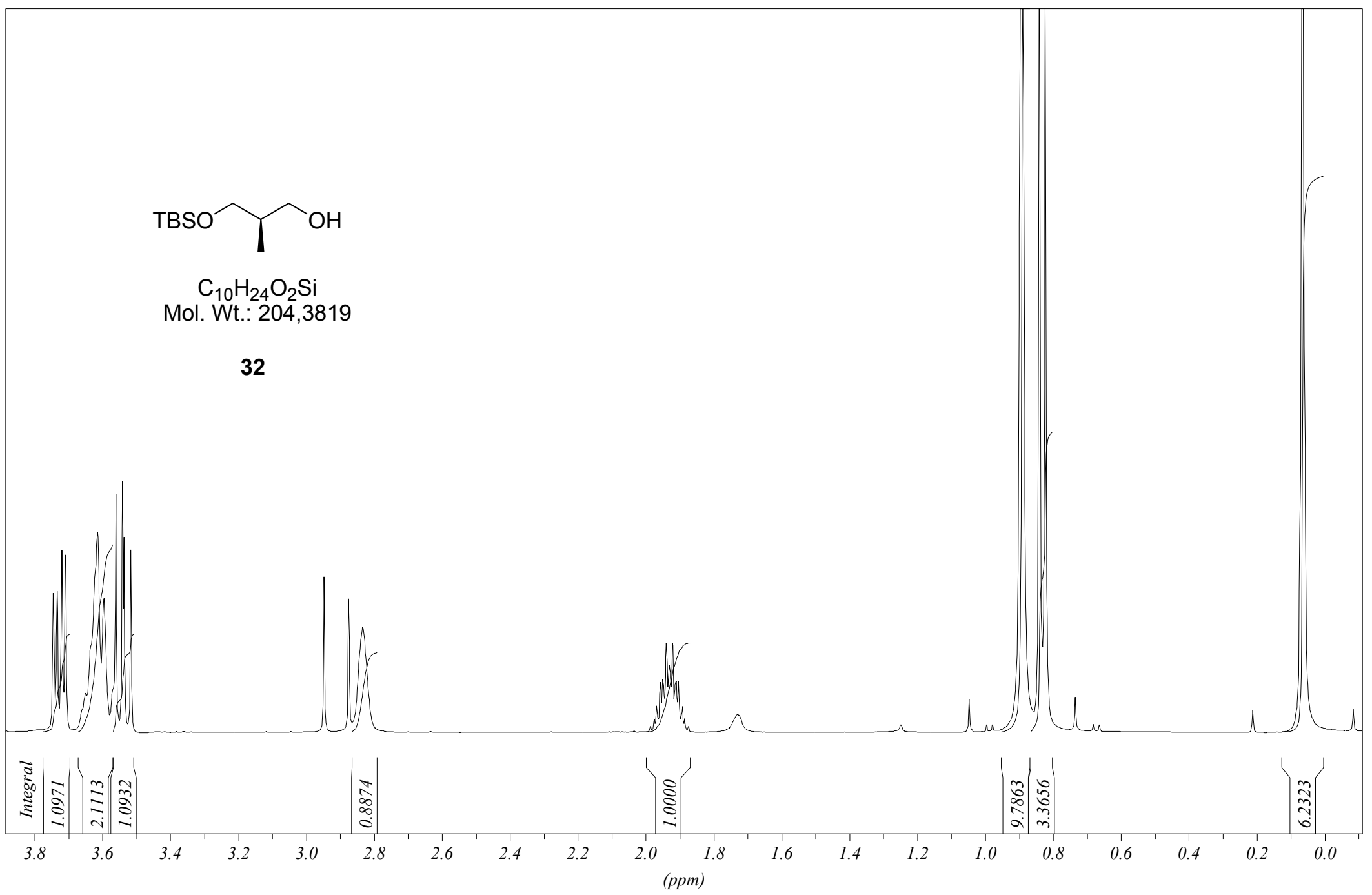




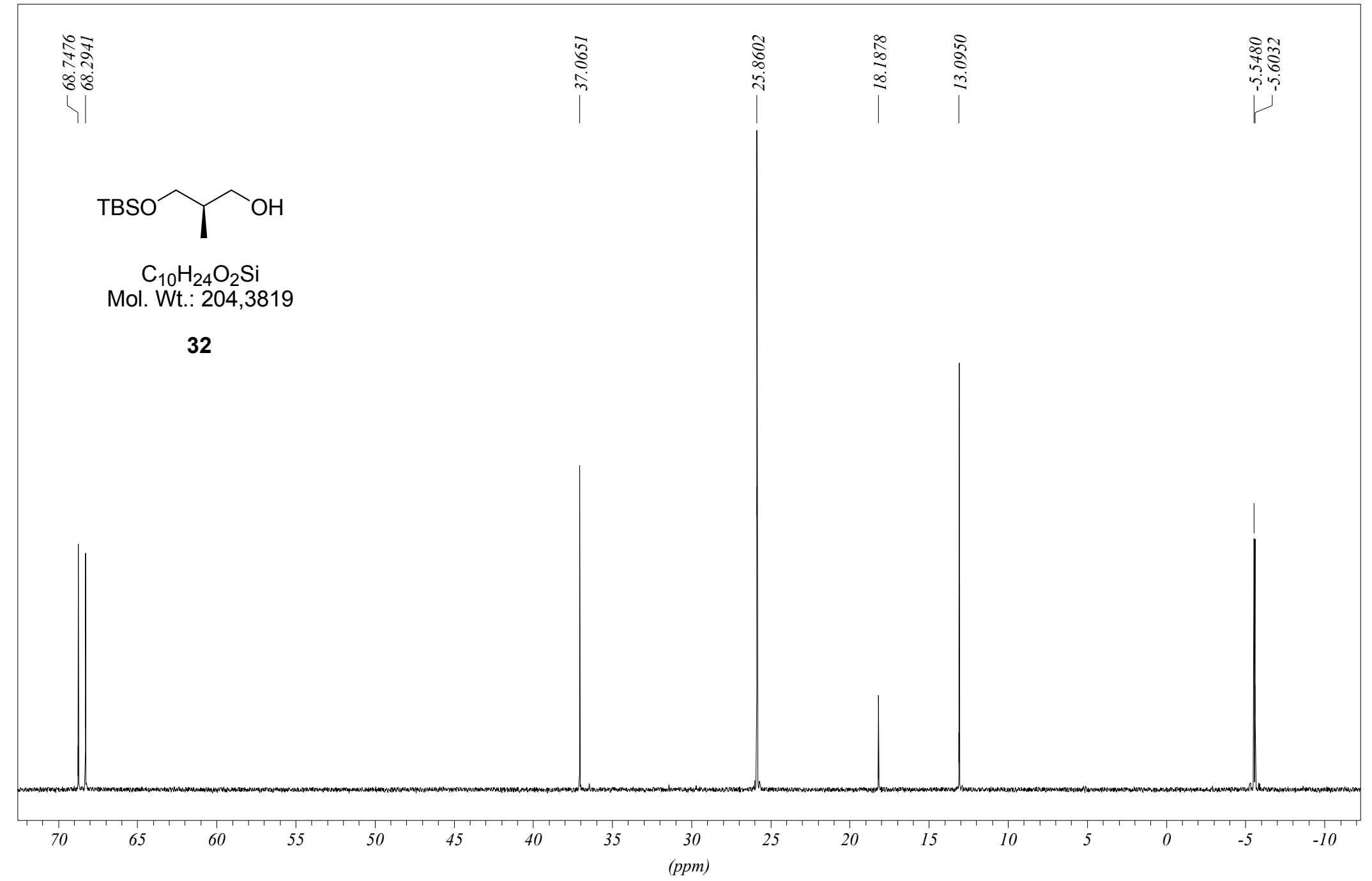




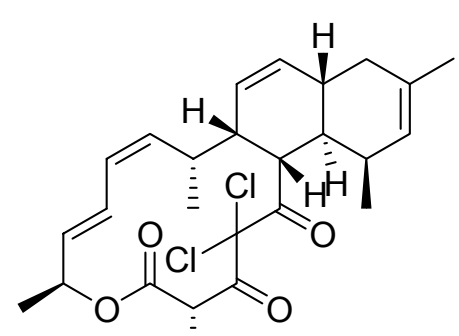

$\mathrm{C}_{26} \mathrm{H}_{32} \mathrm{Cl}_{2} \mathrm{O}_{4}$ Mol. Wt.: 479,4359

(3)

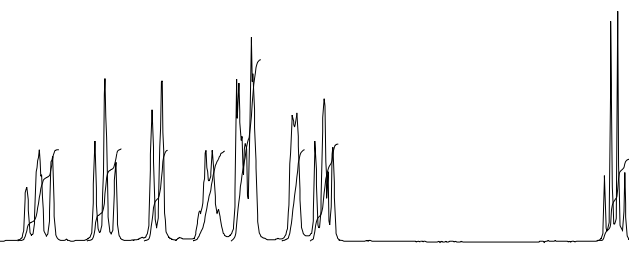

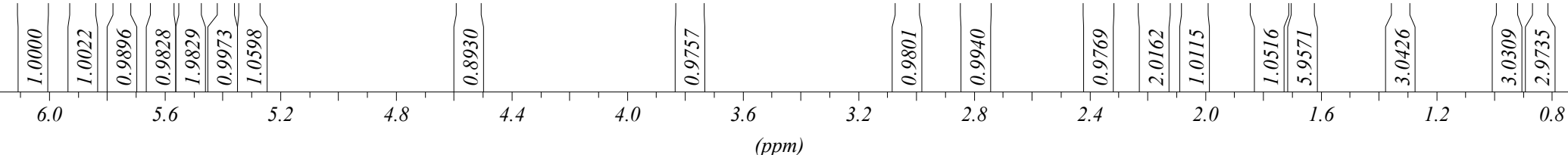




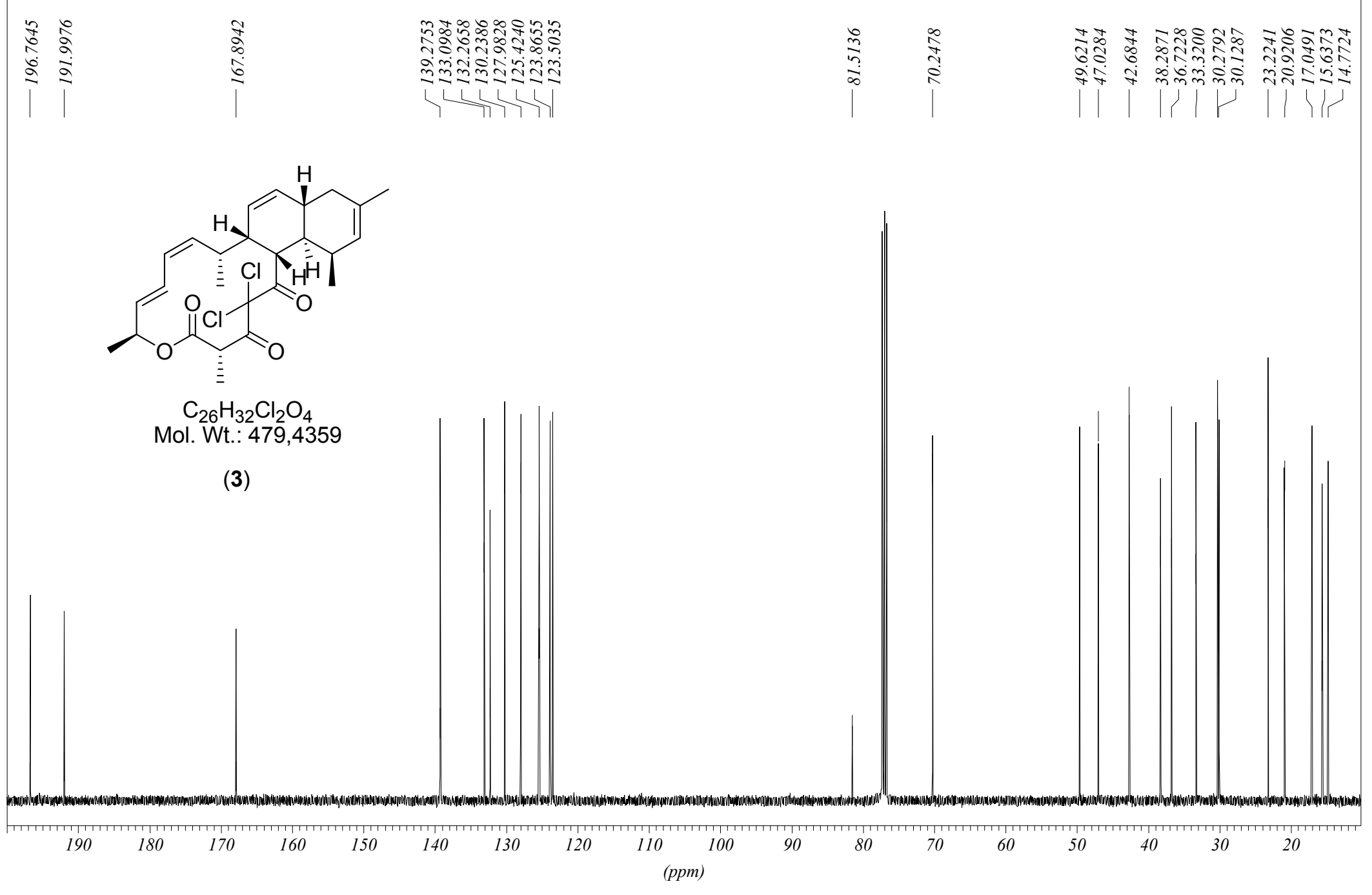




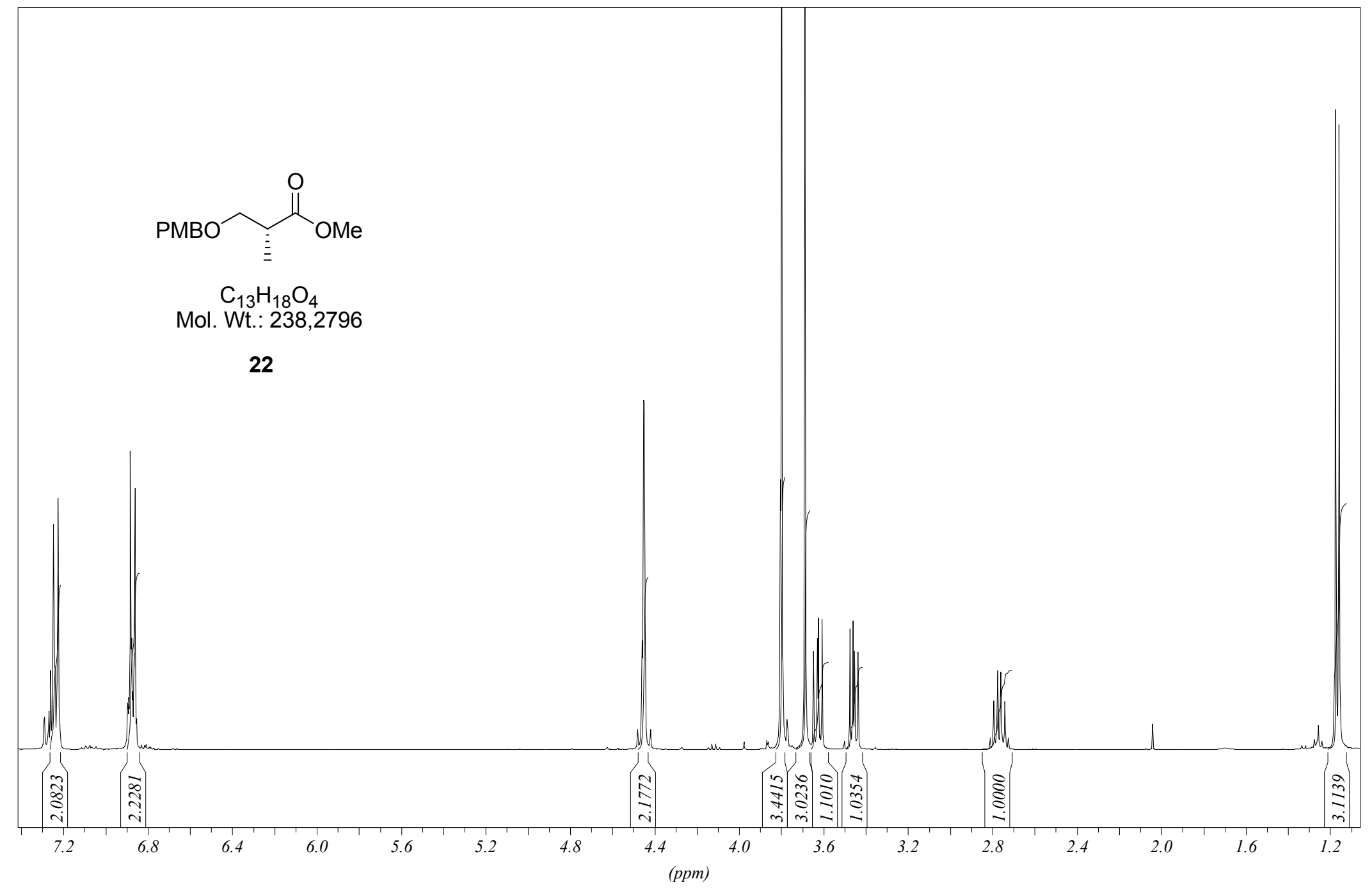




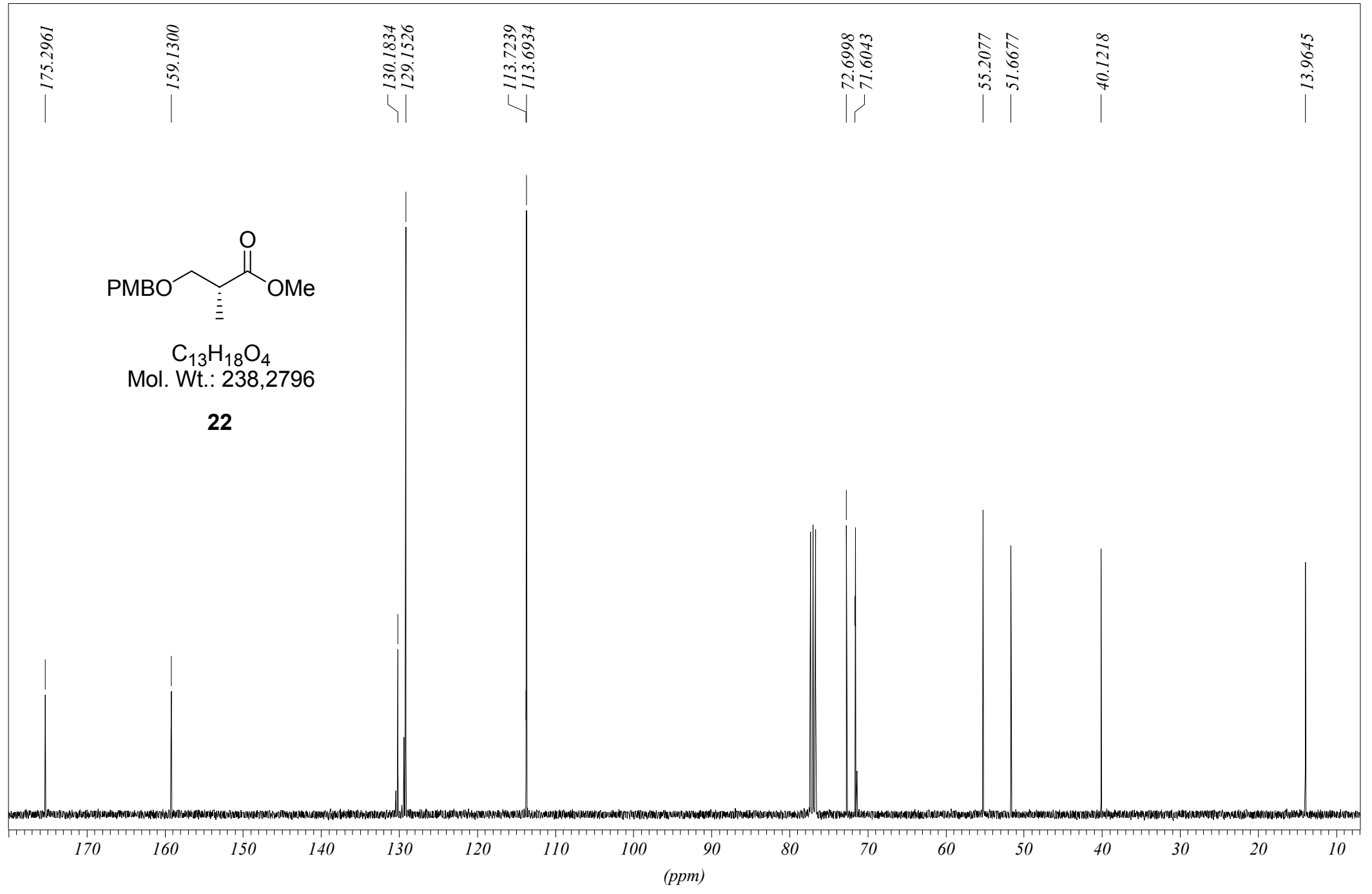




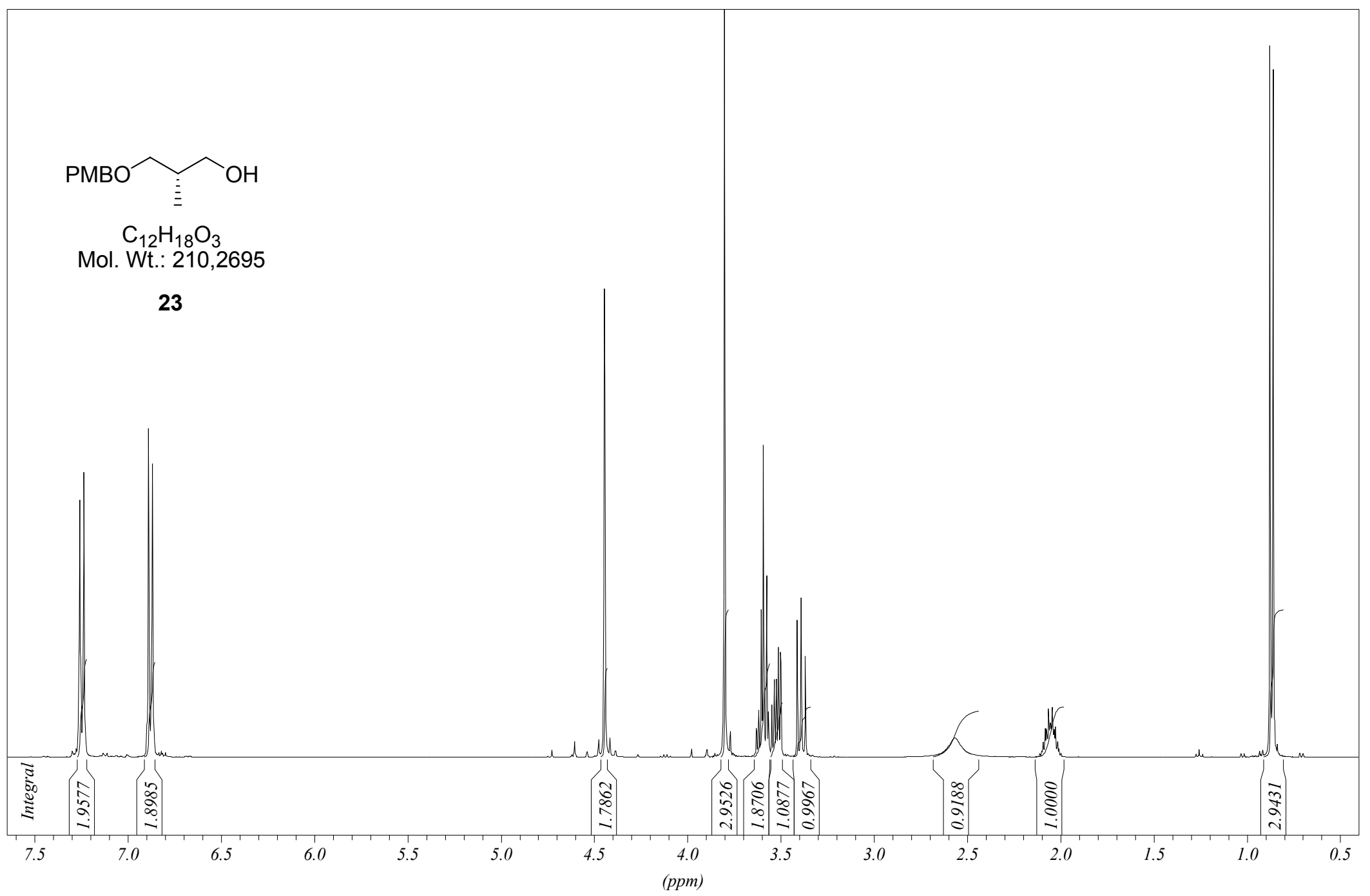




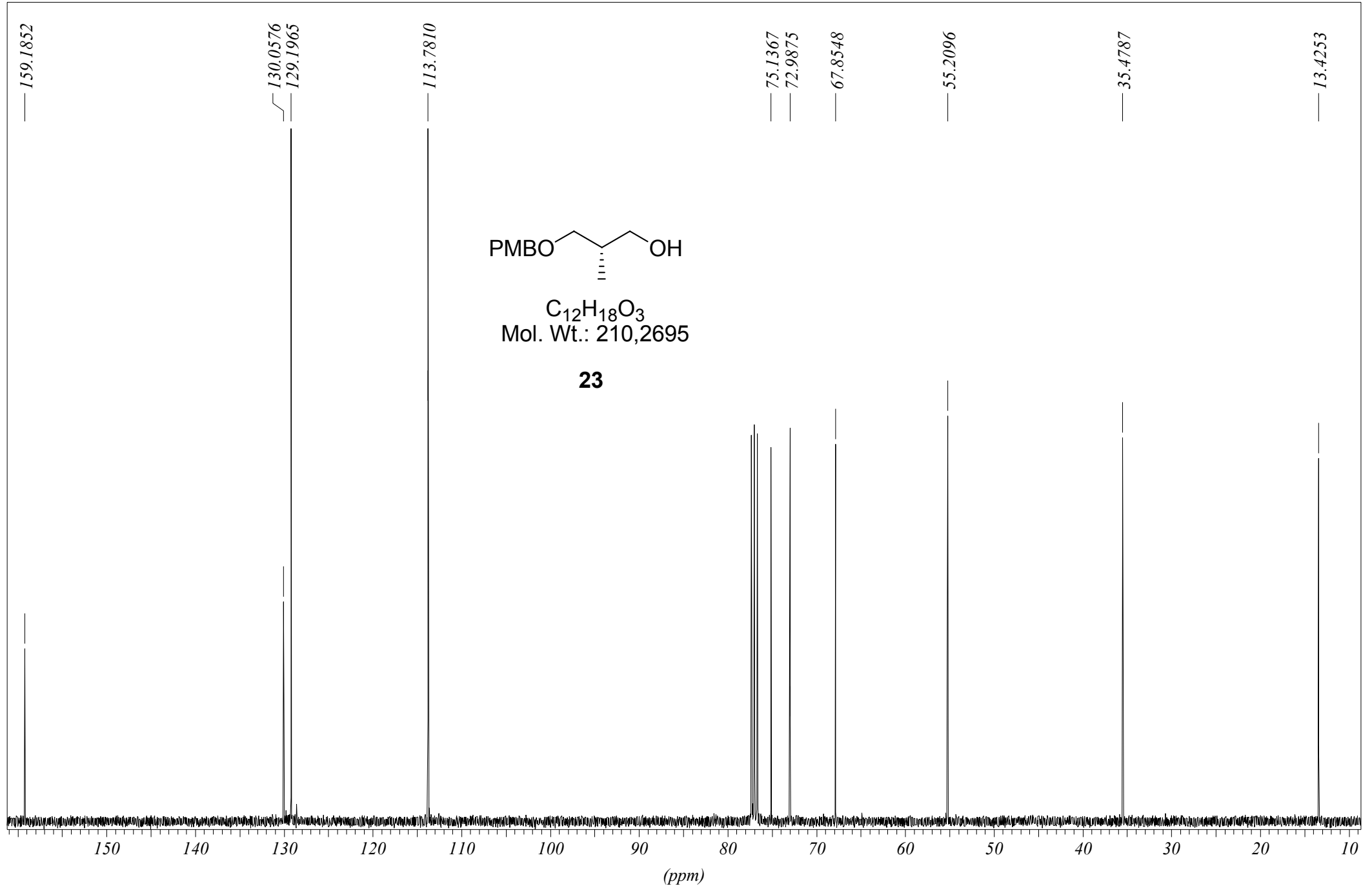




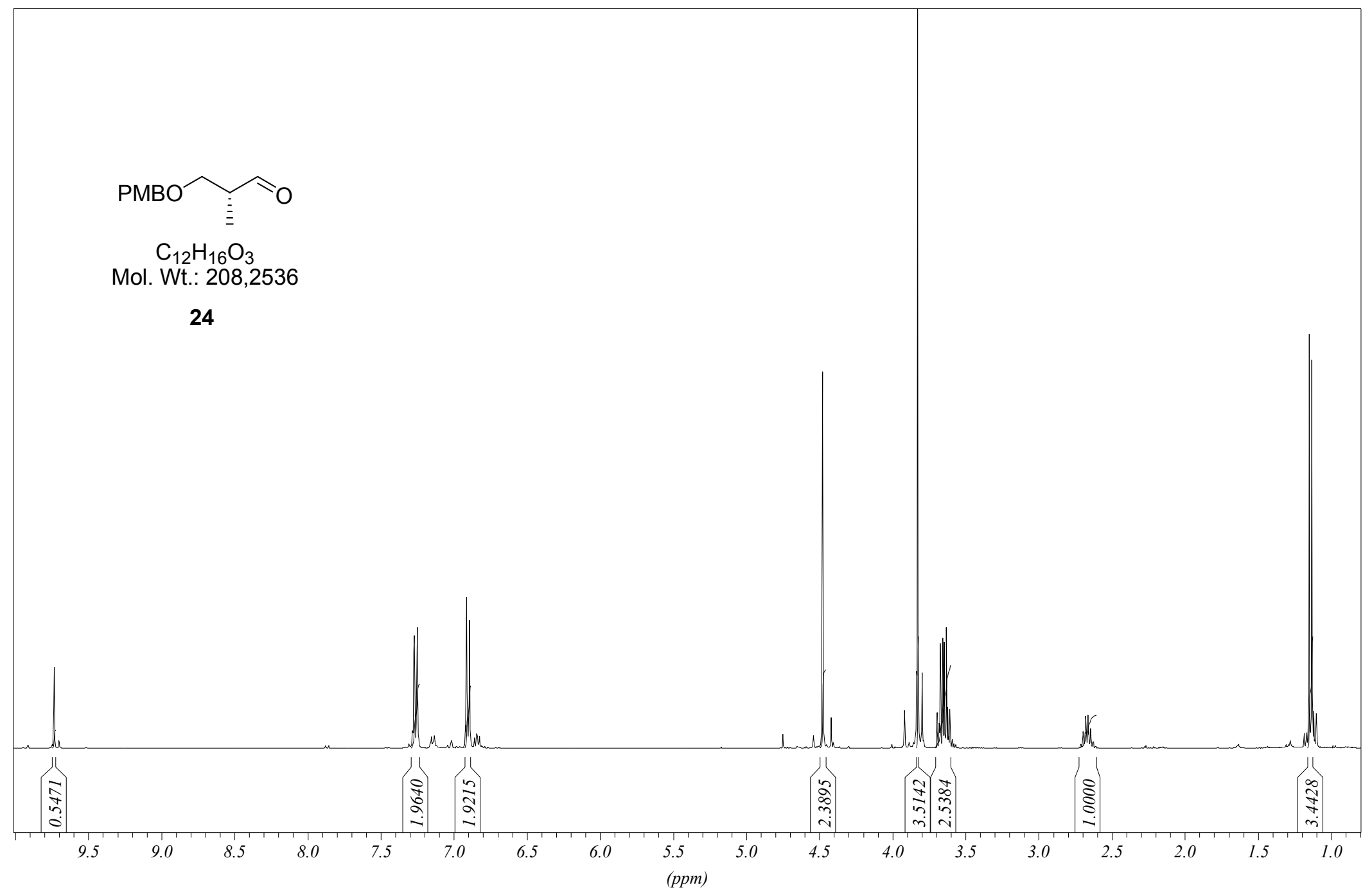




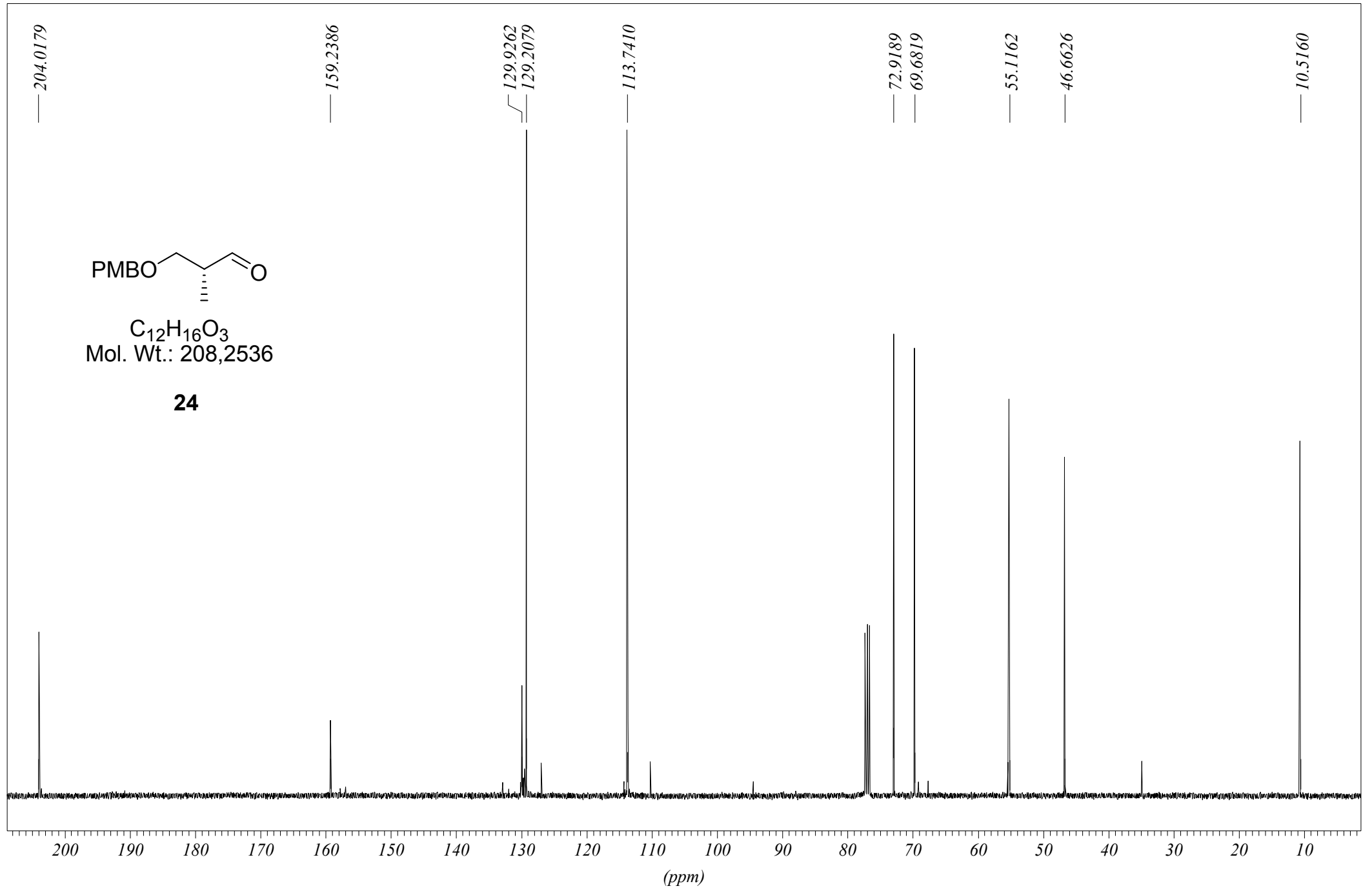




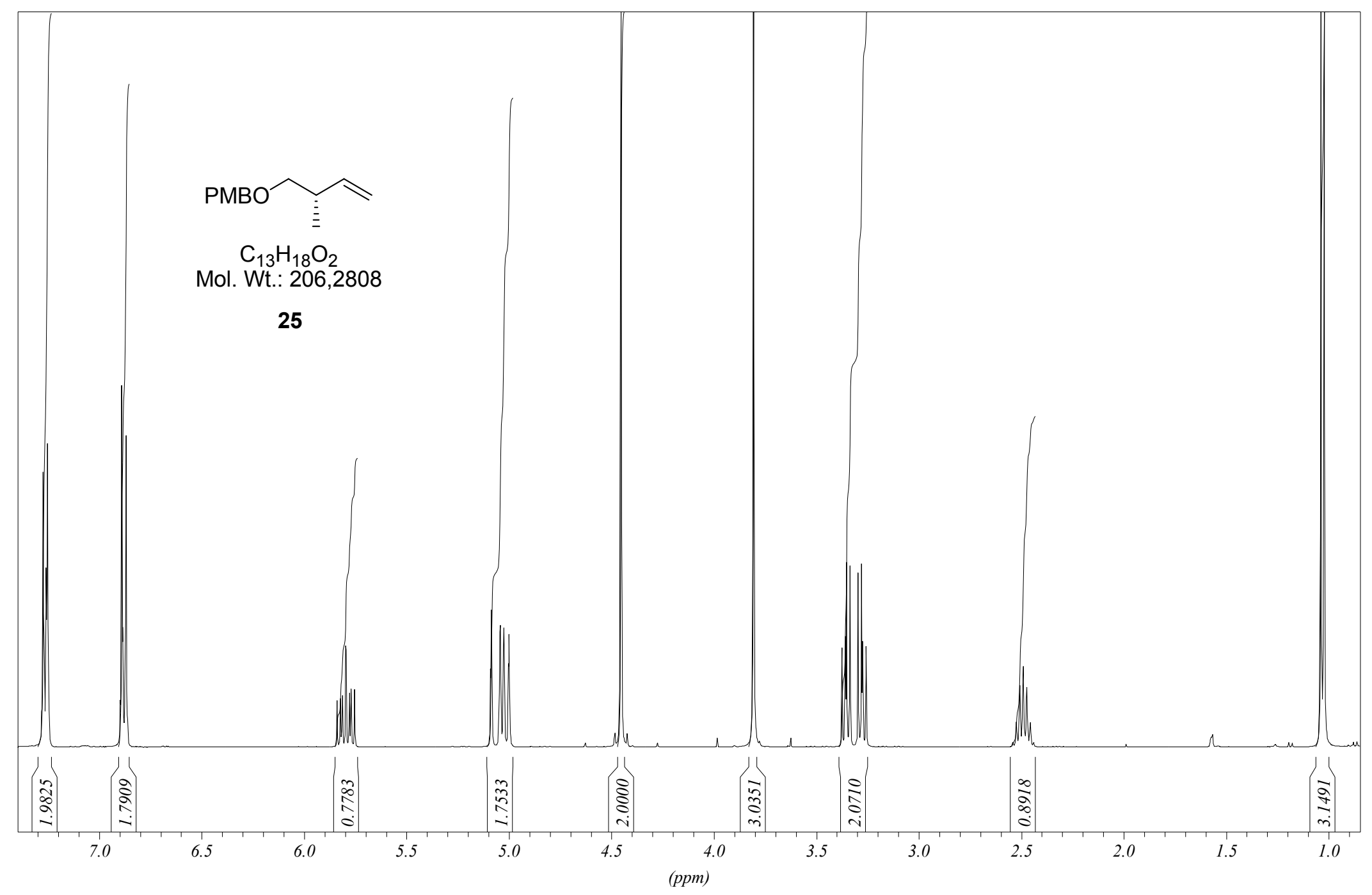




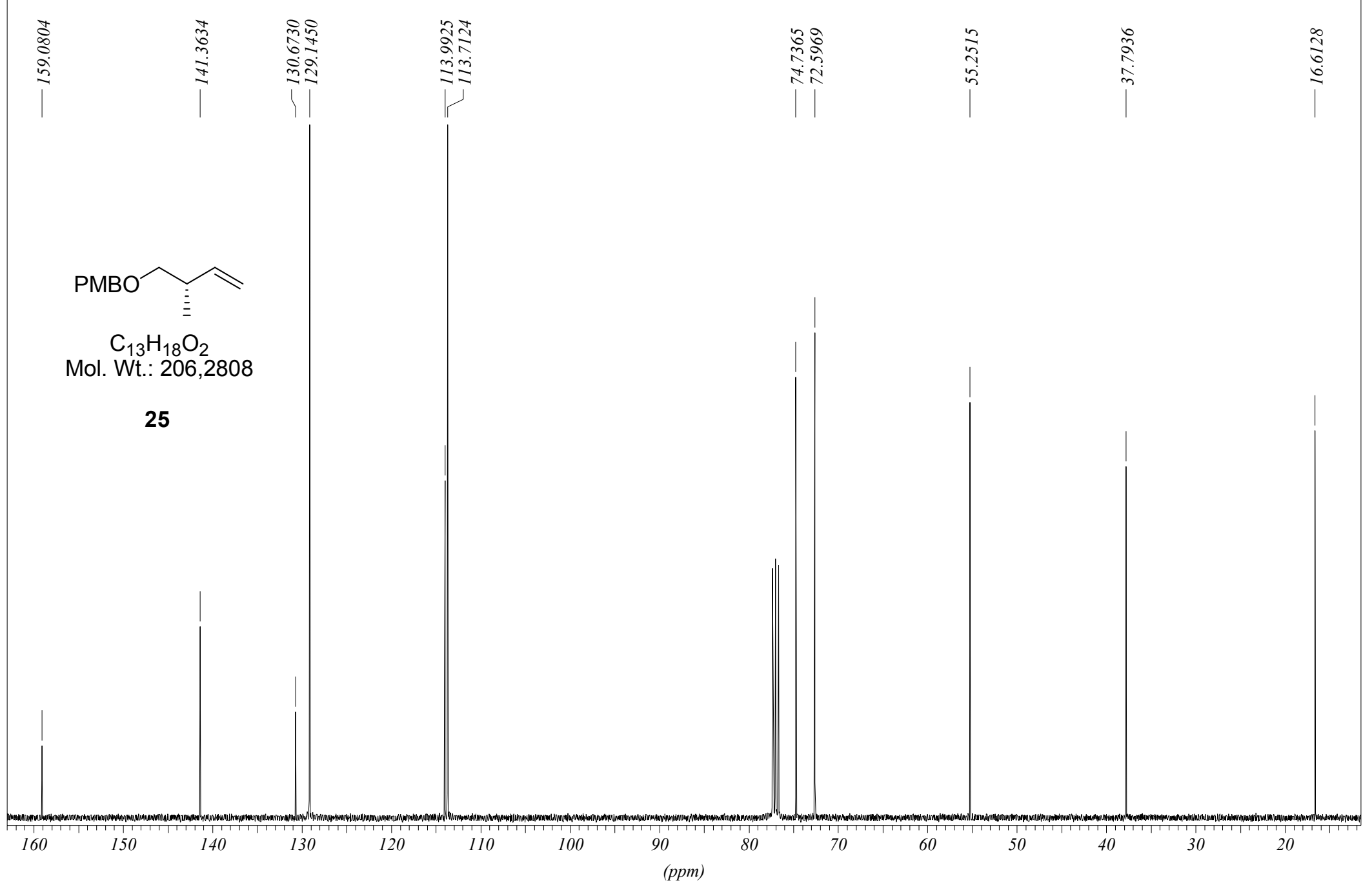




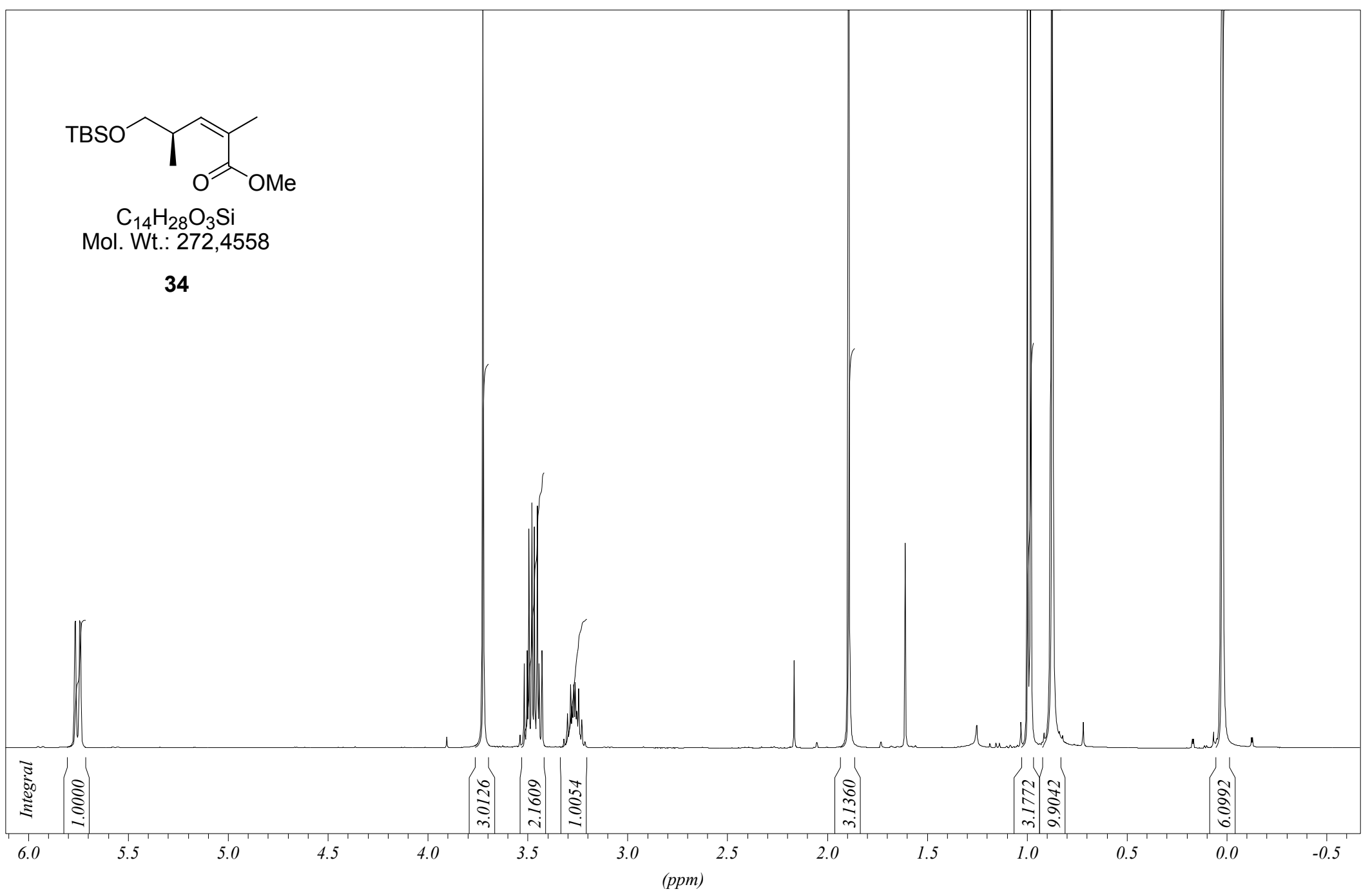




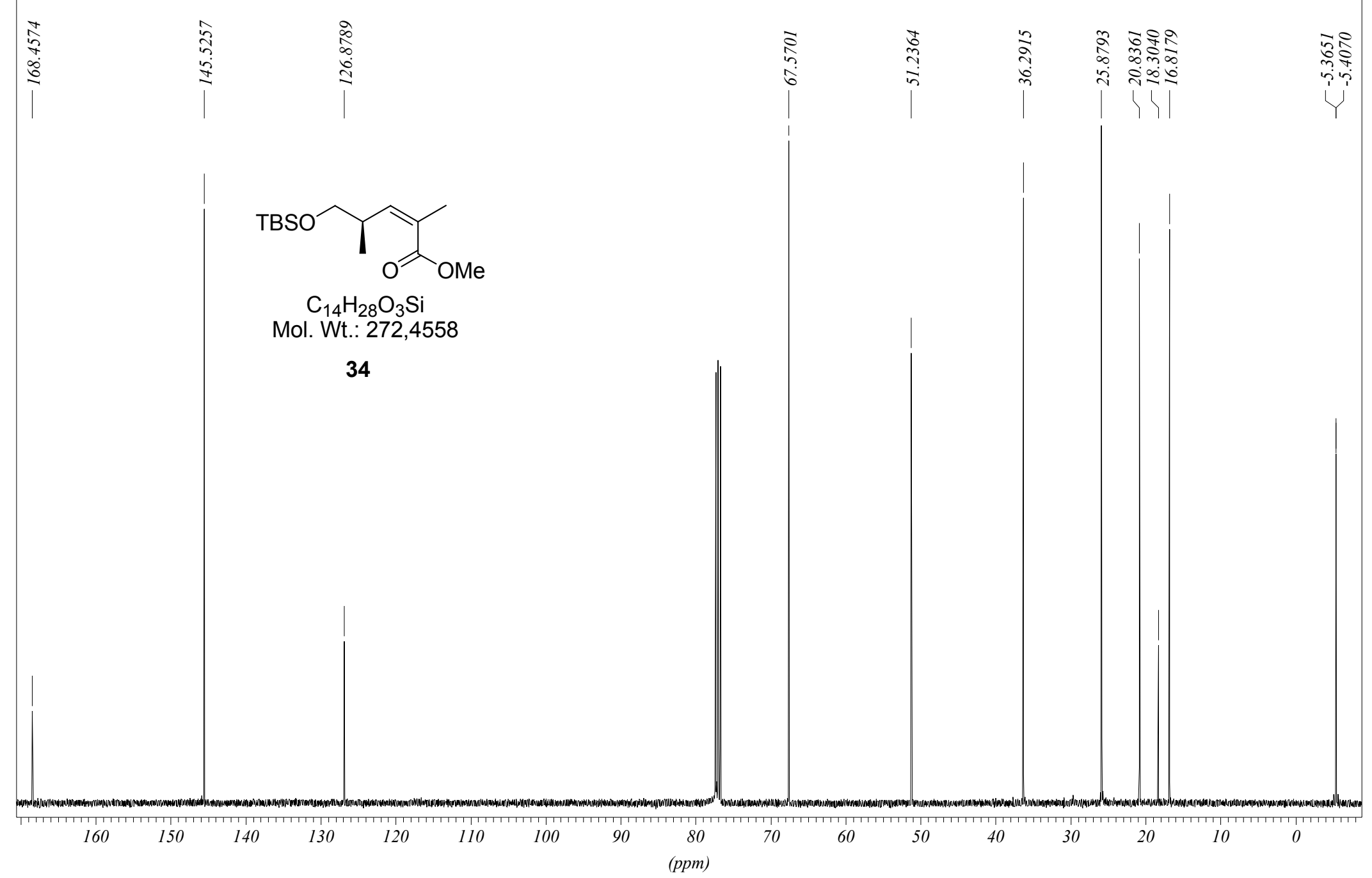




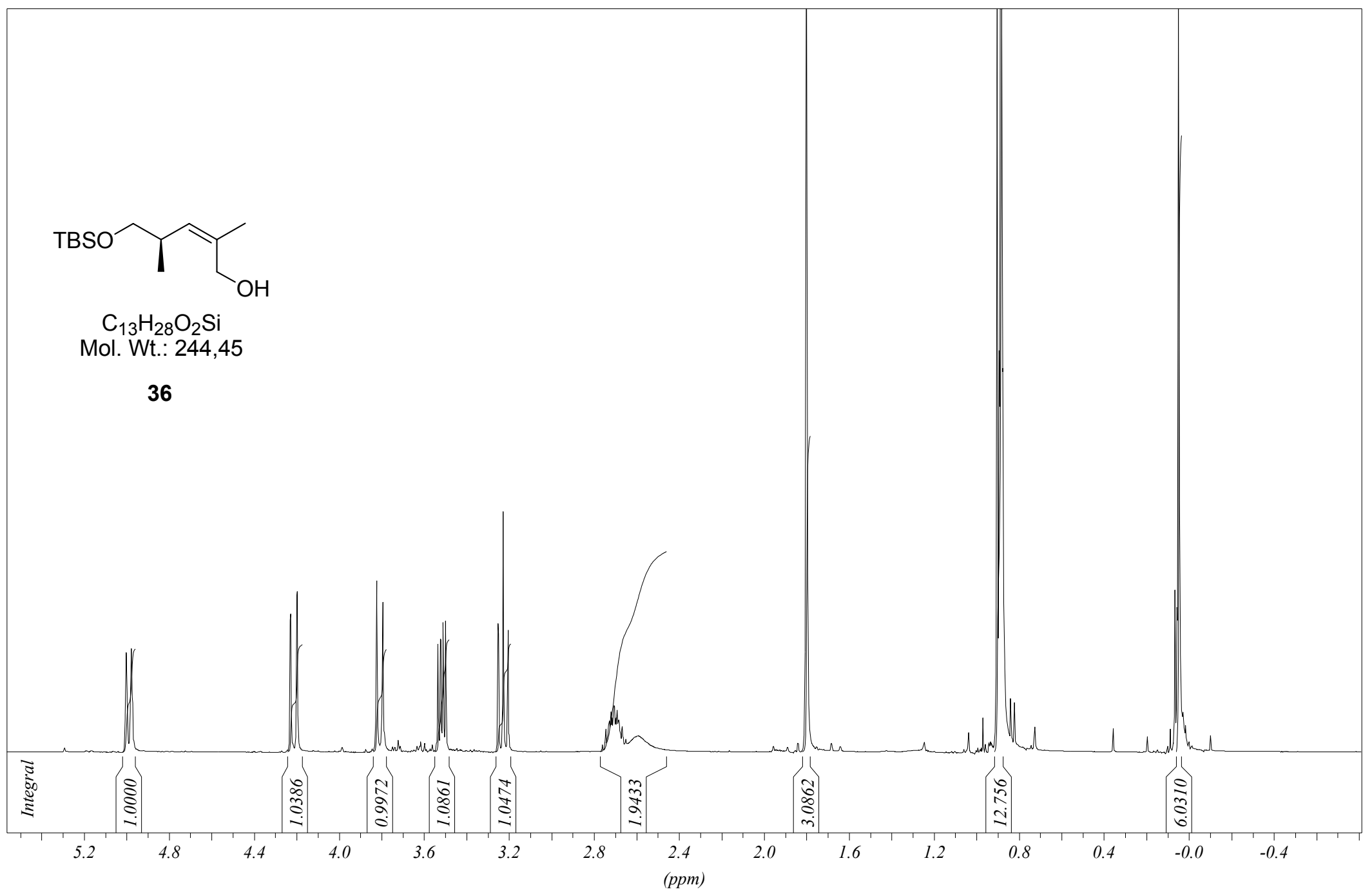




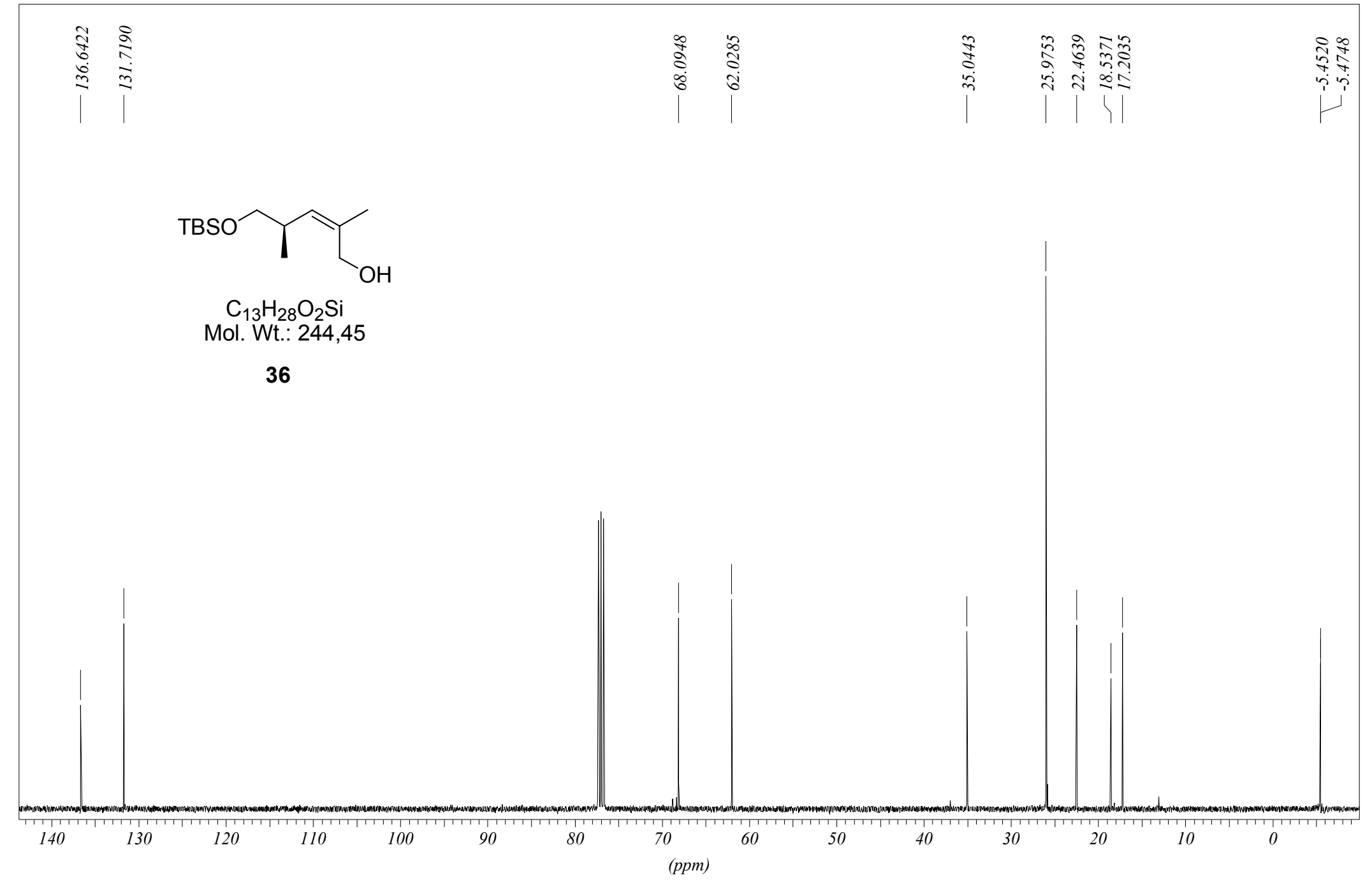




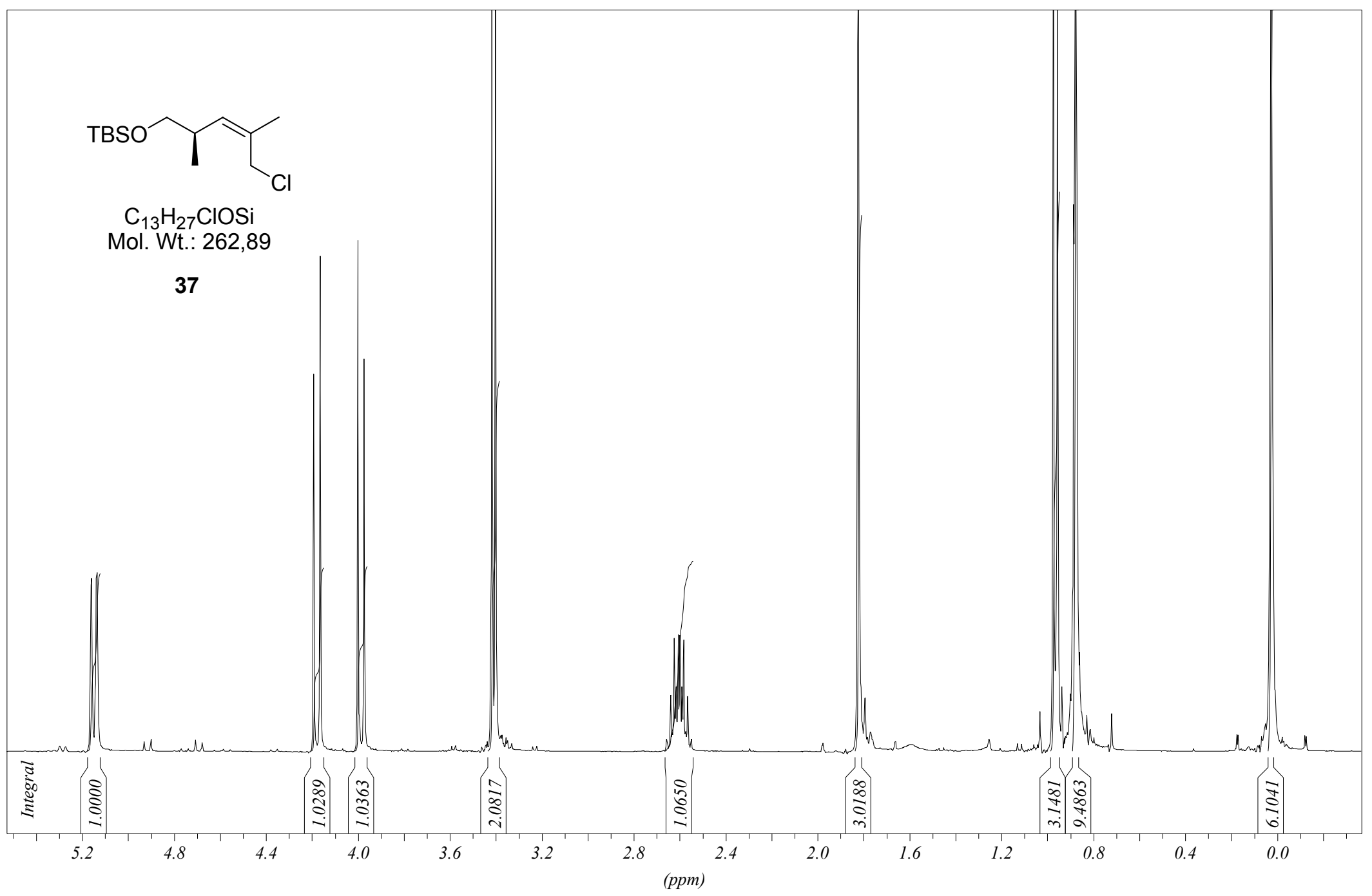




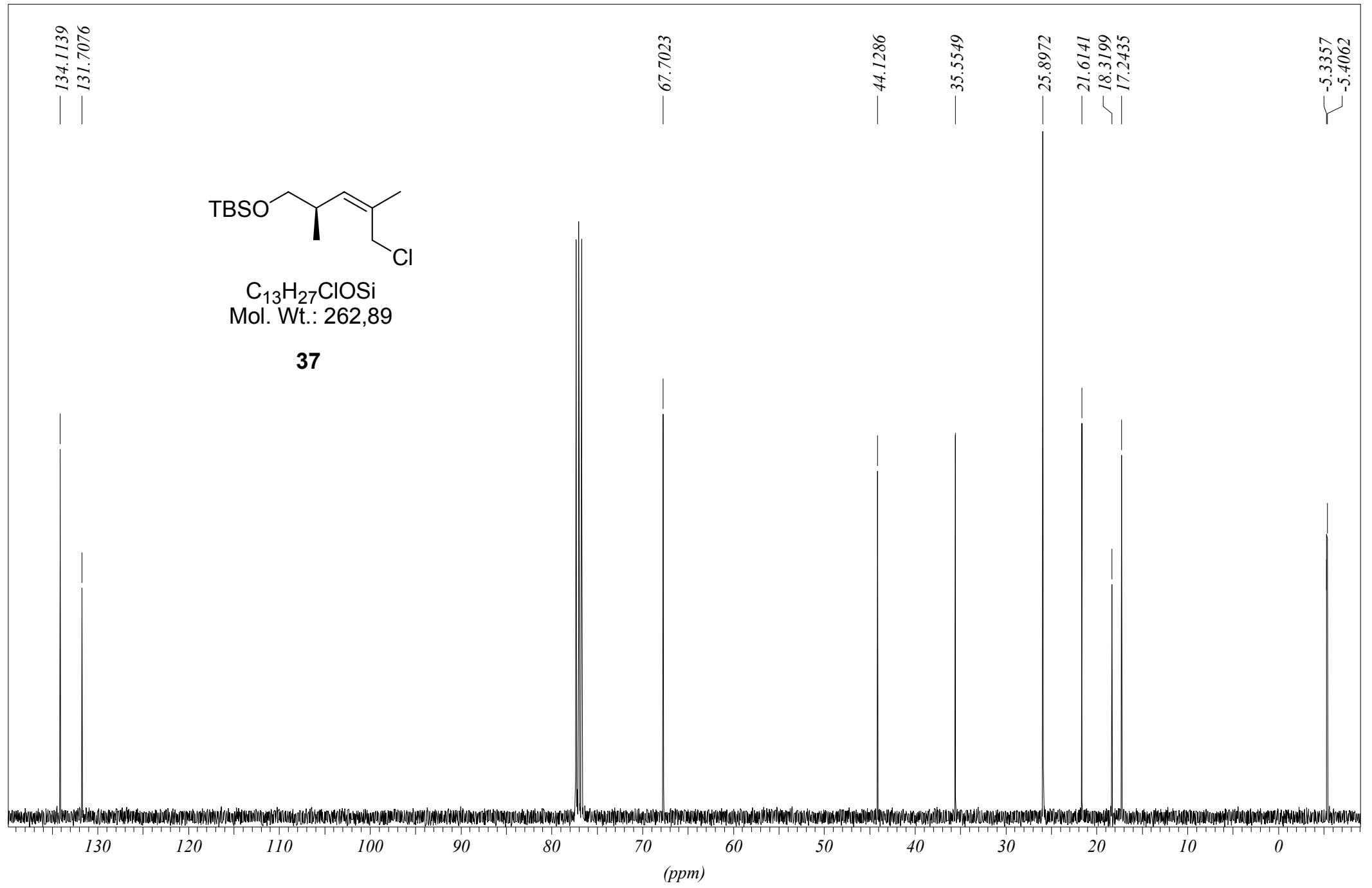




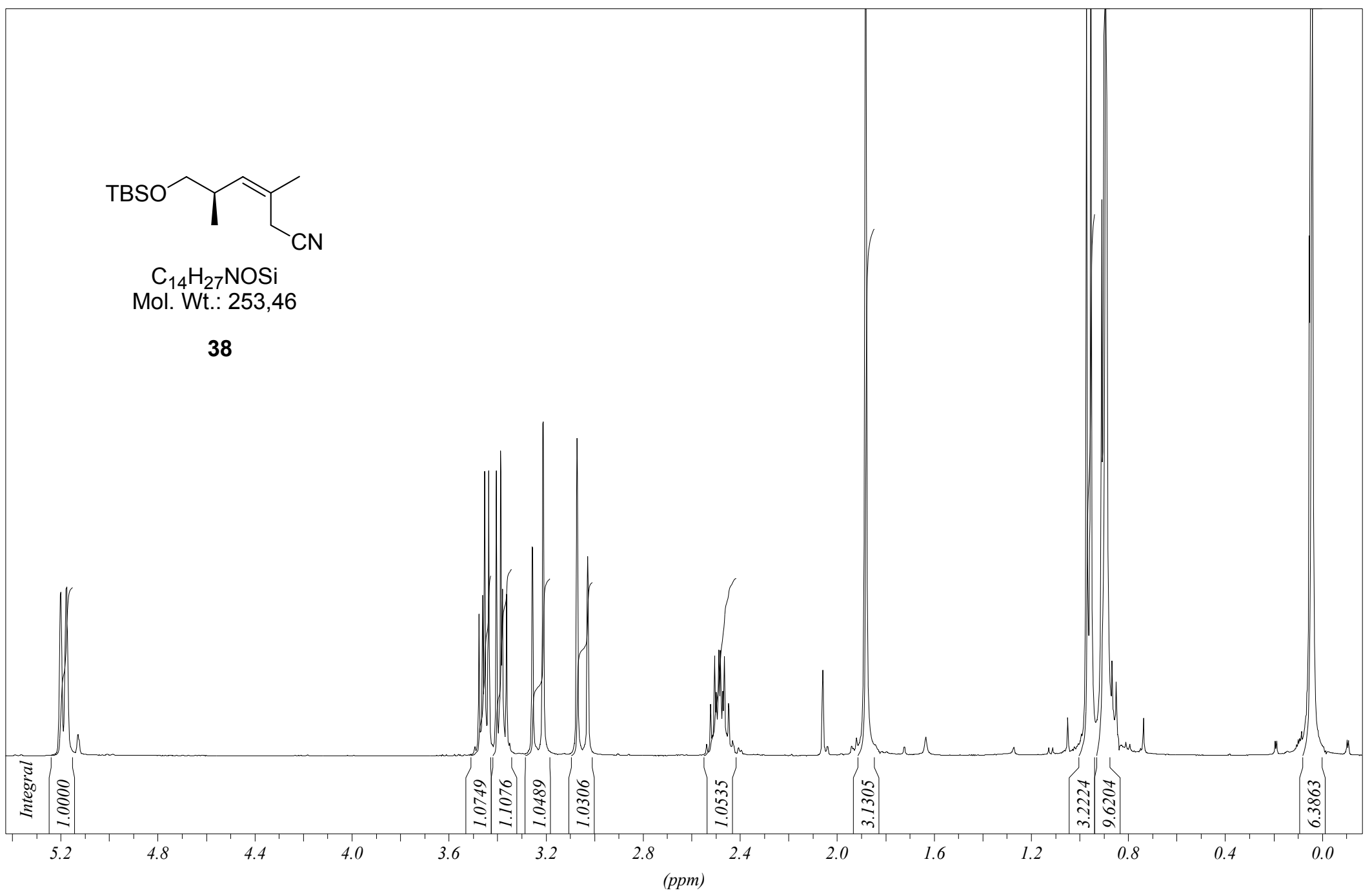




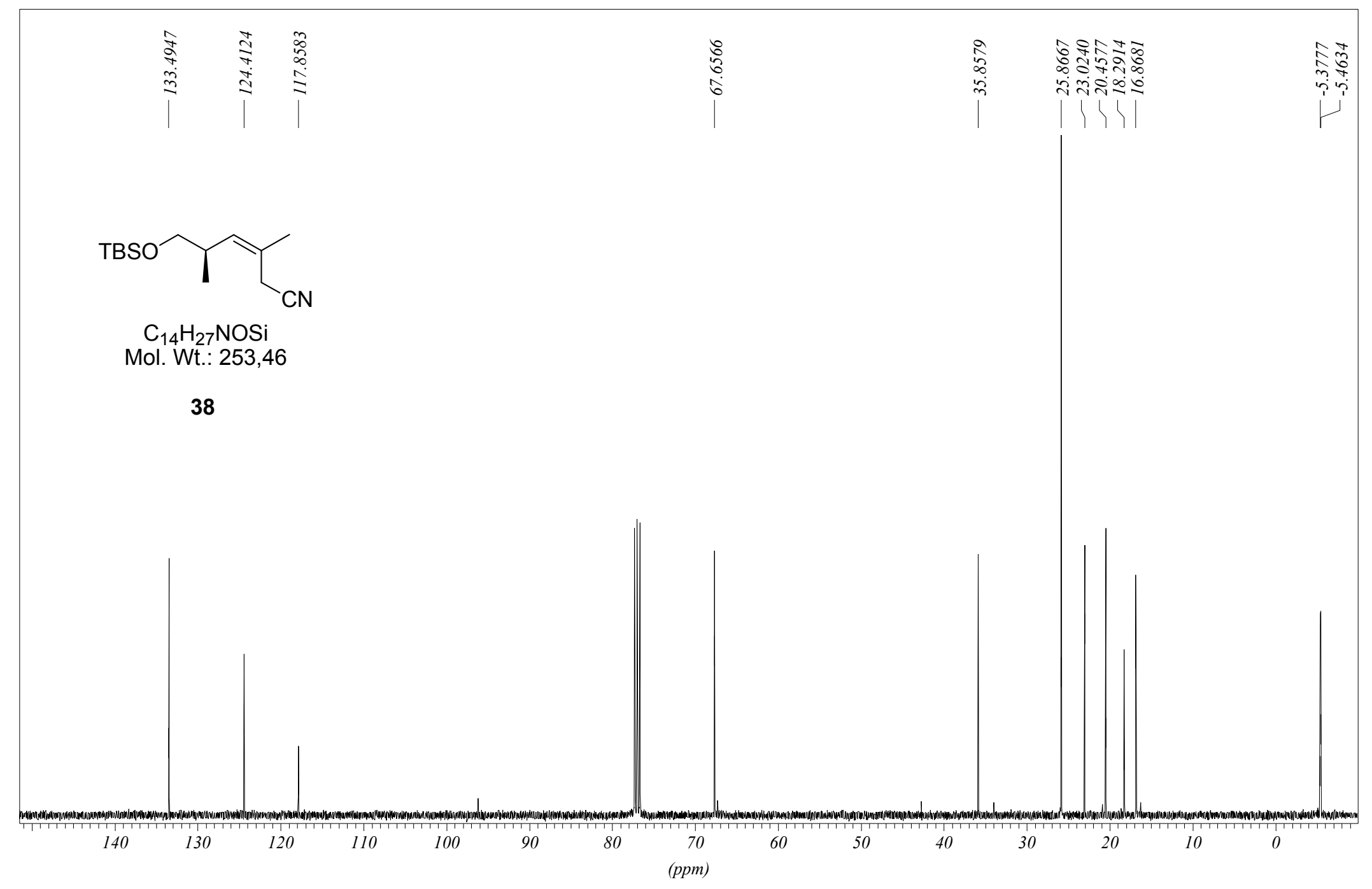




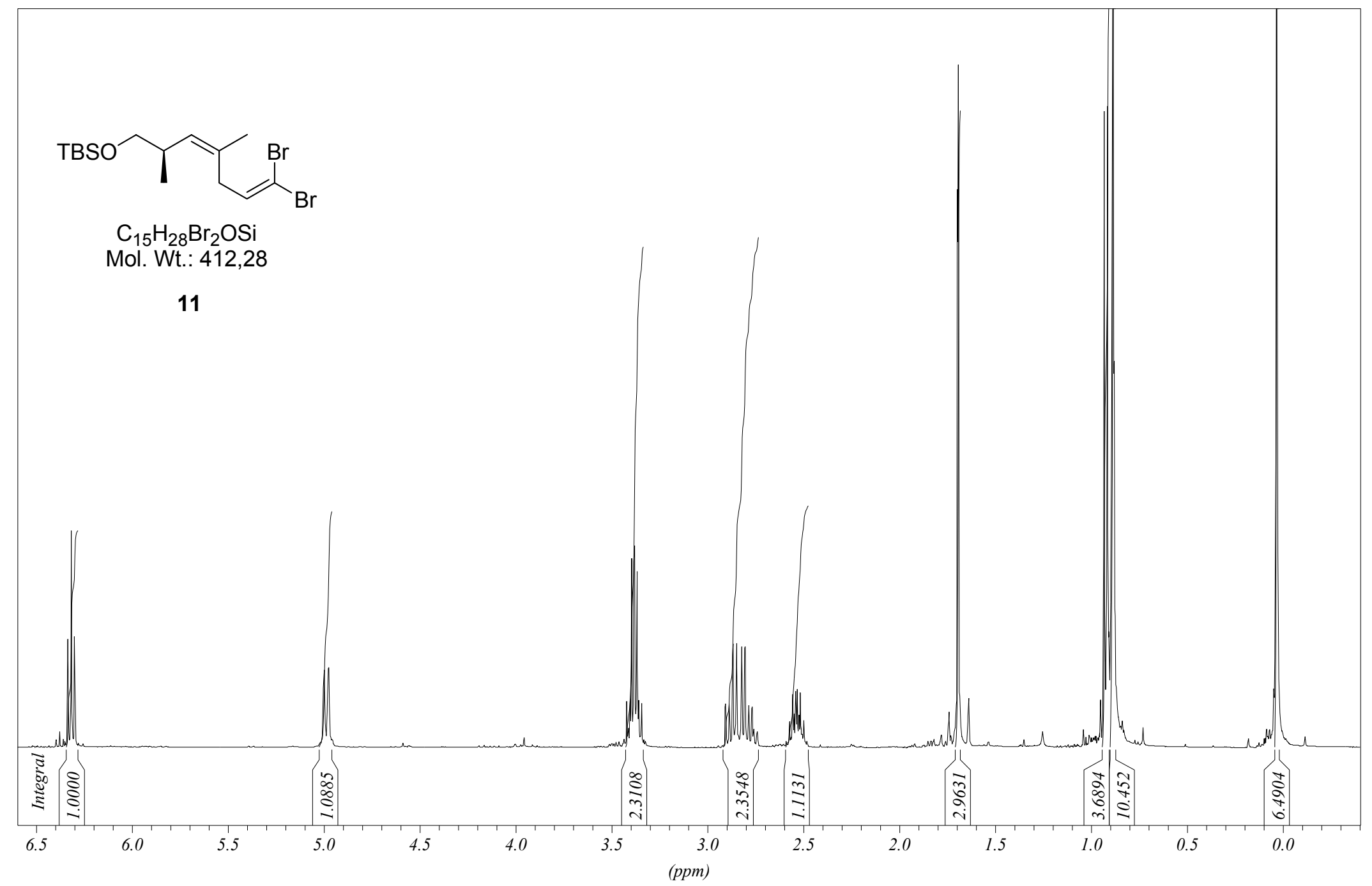




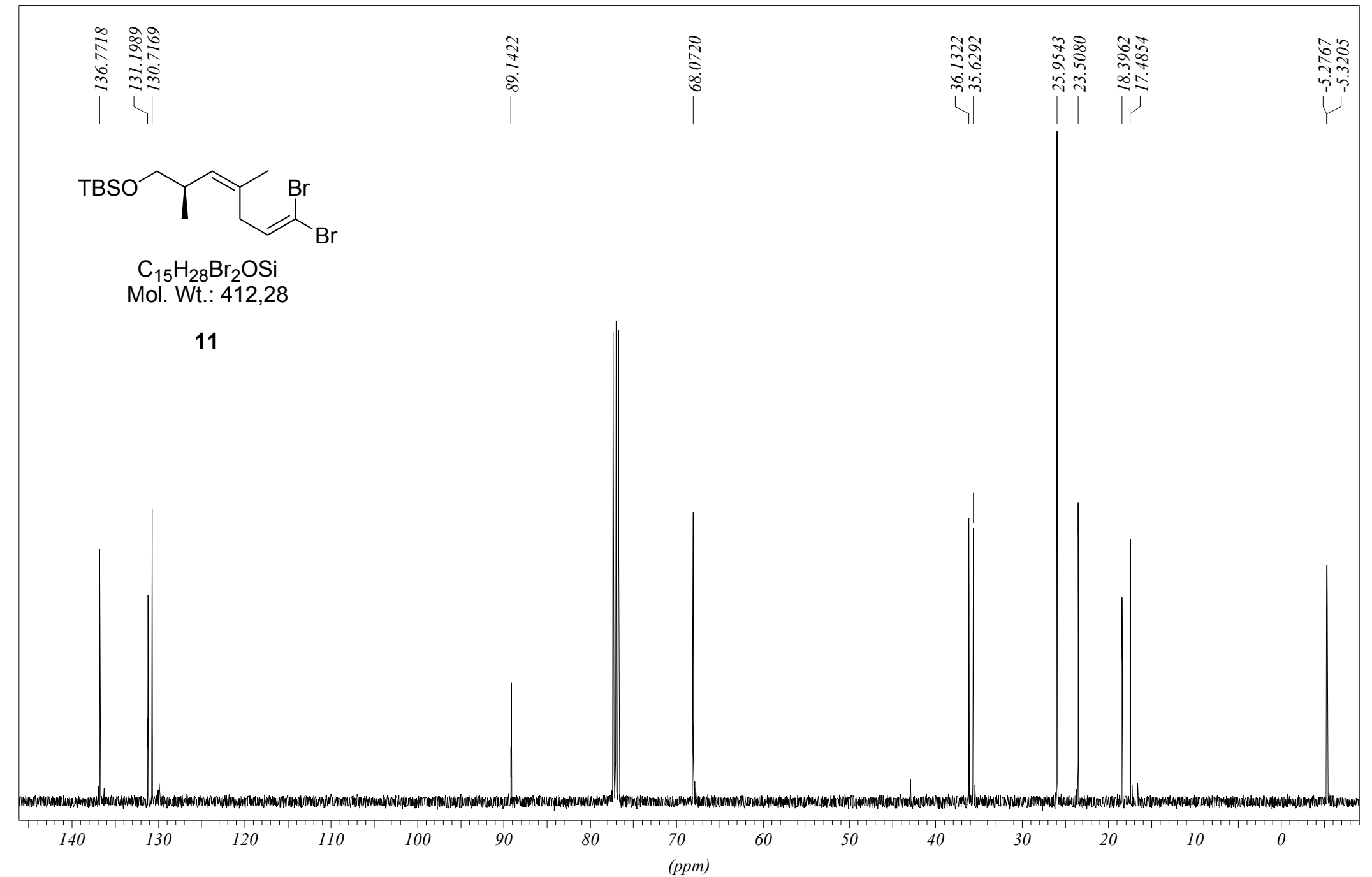




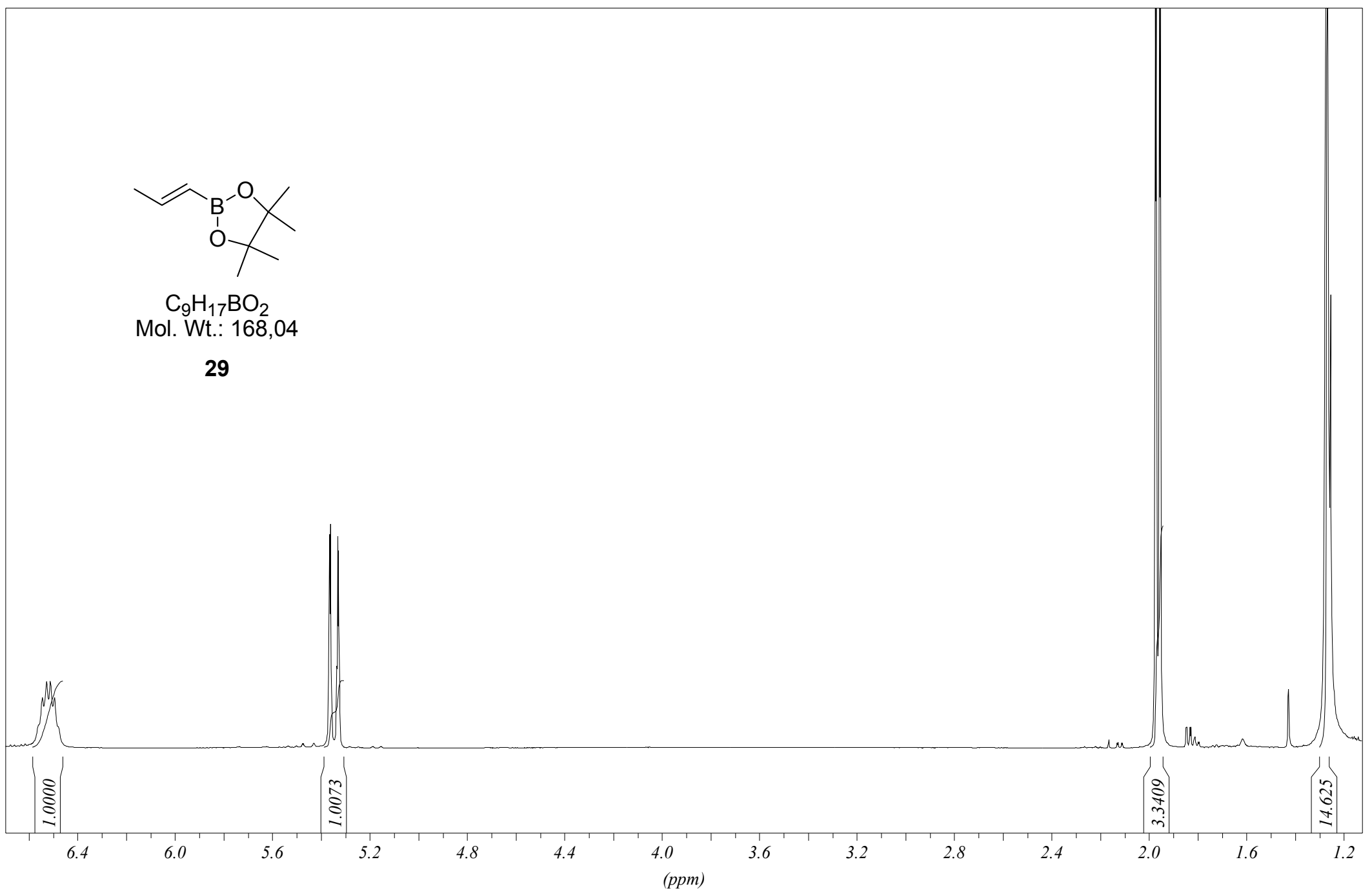




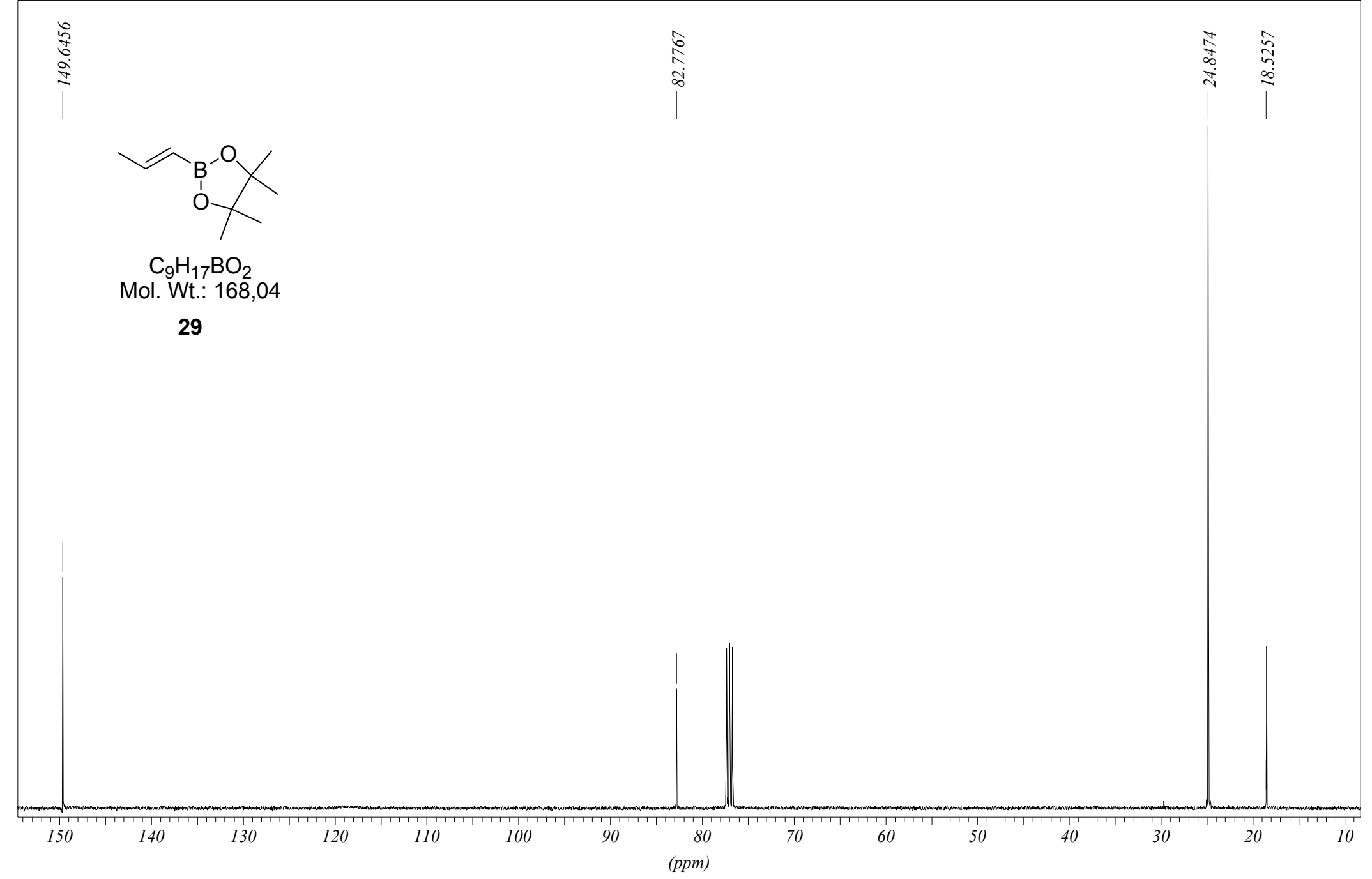




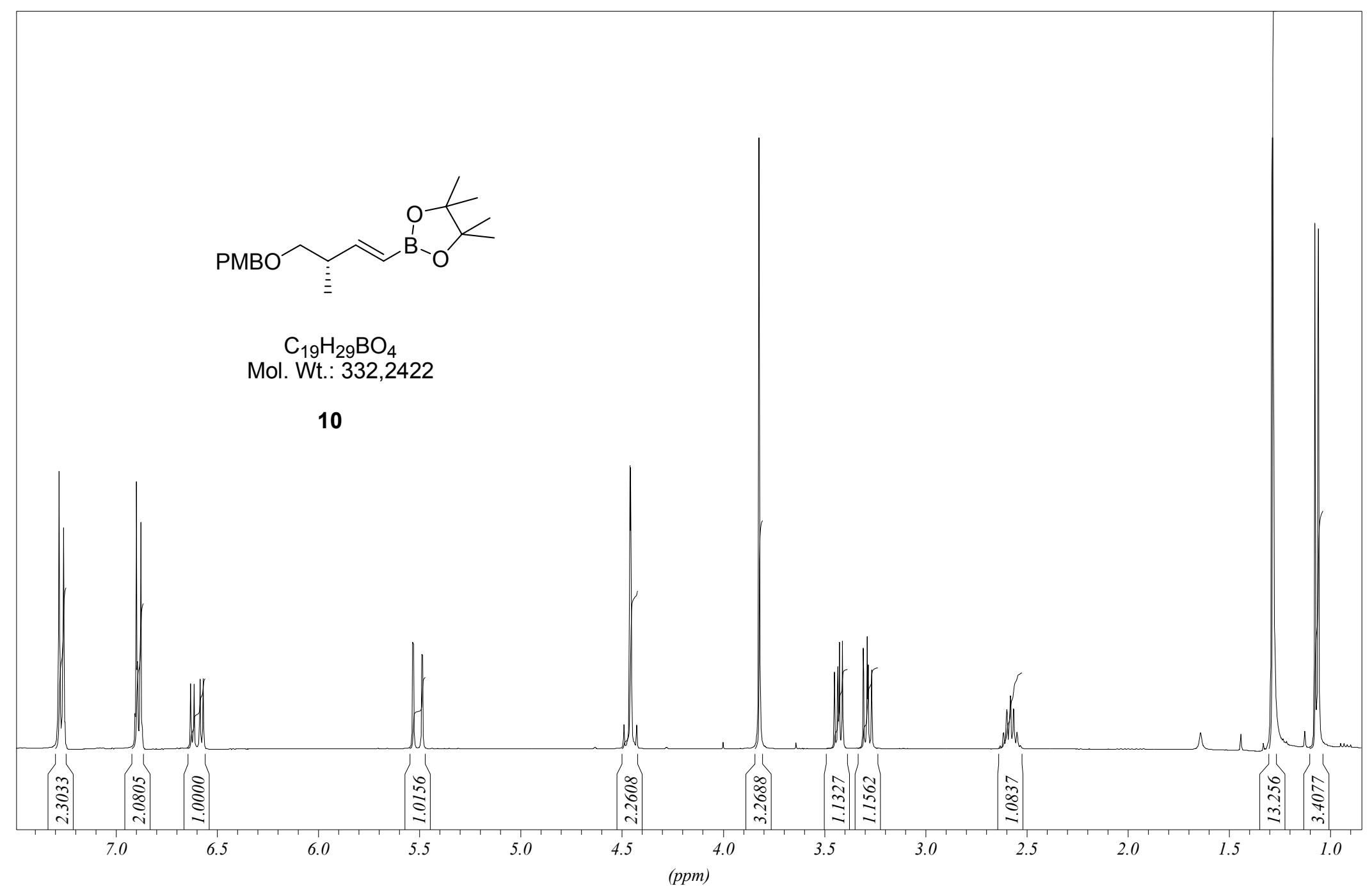




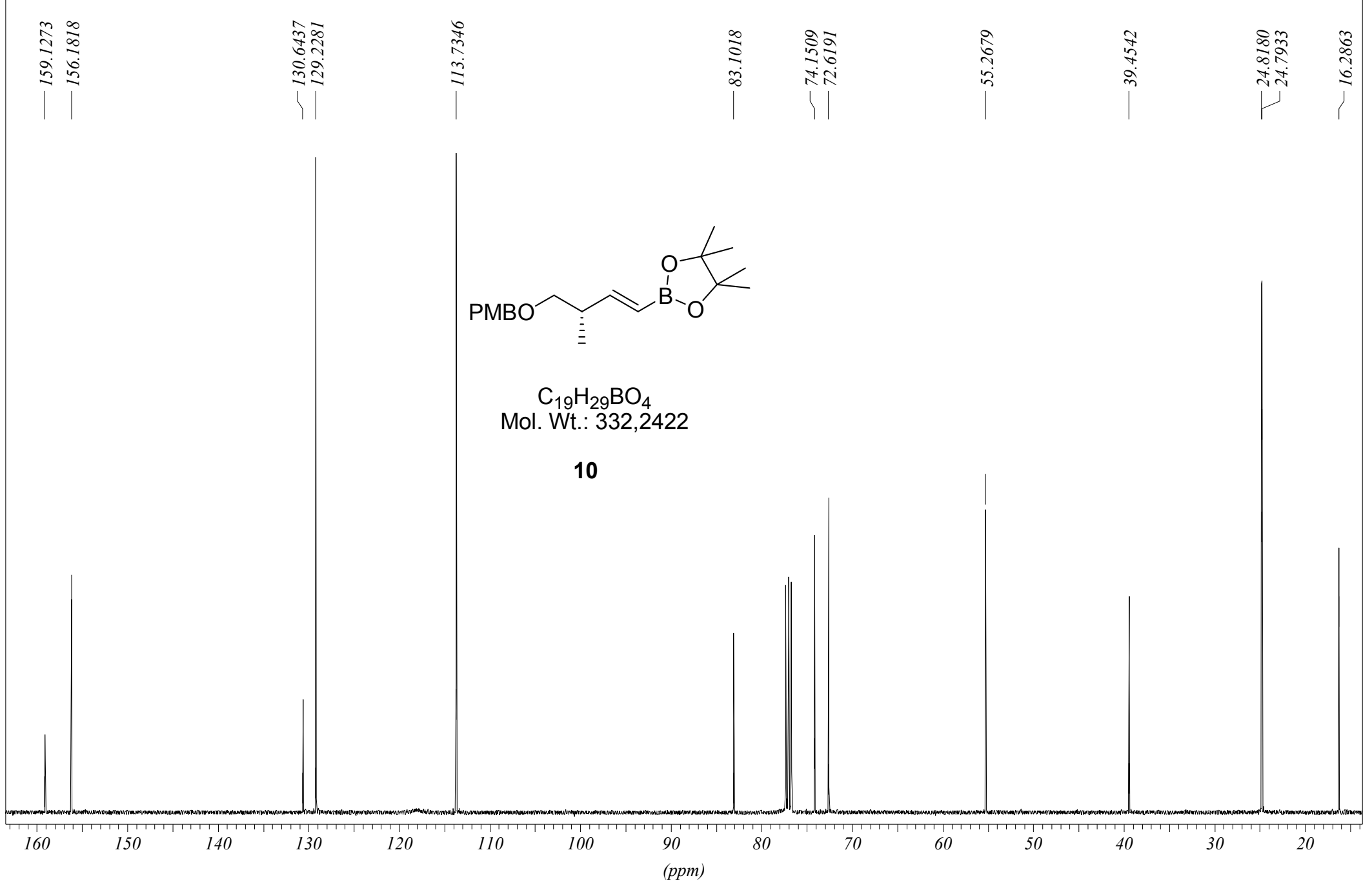




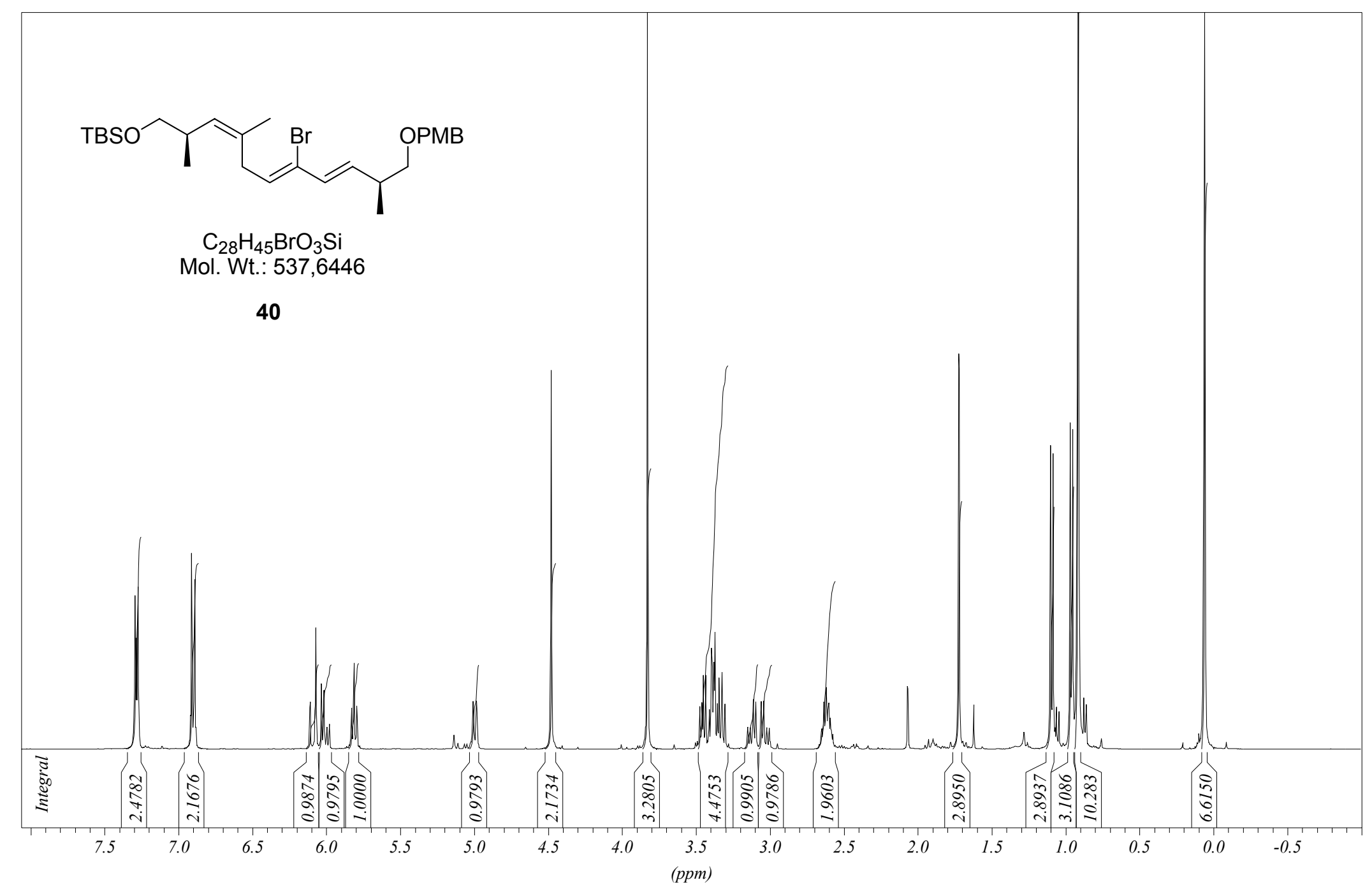




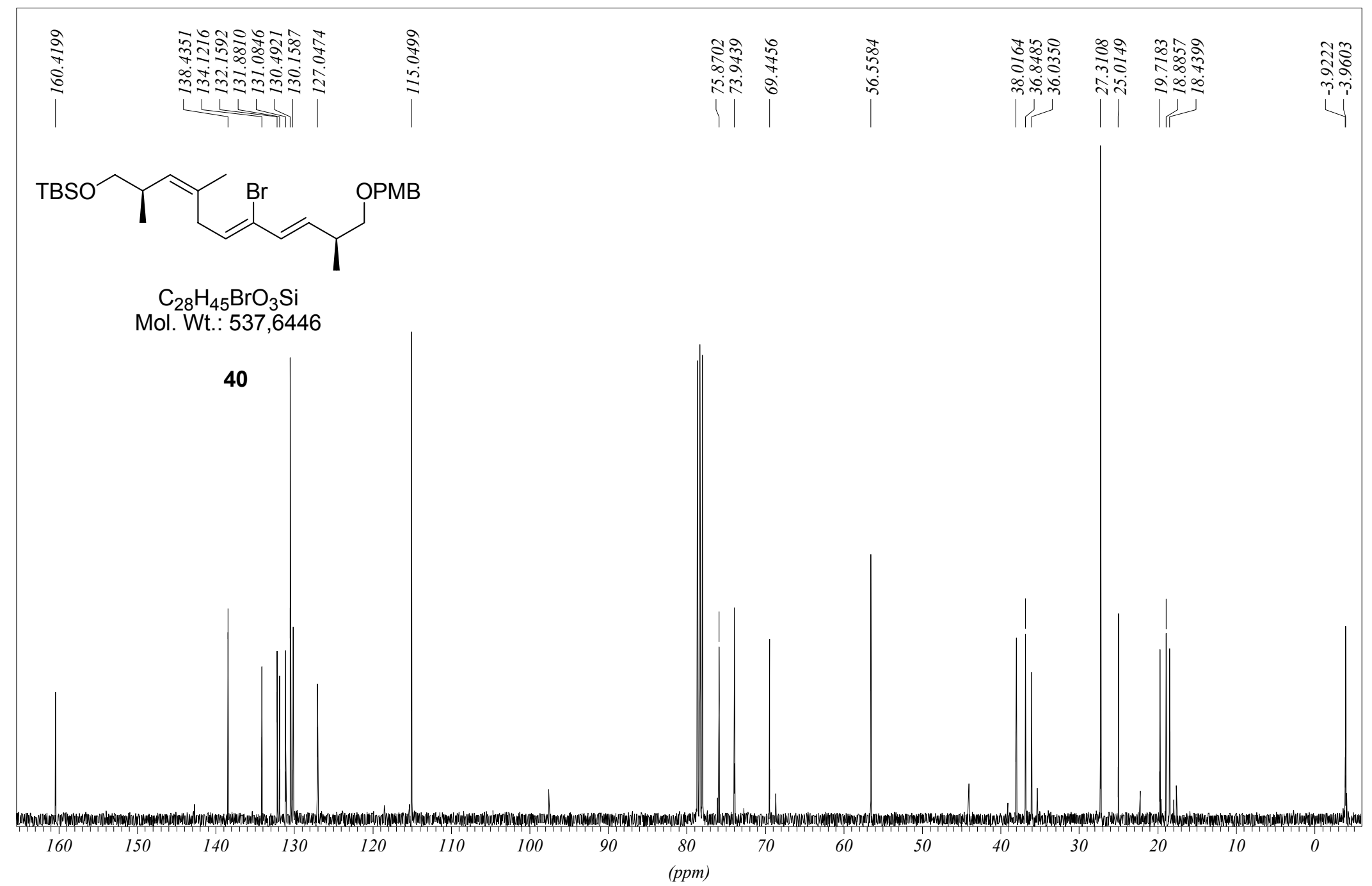




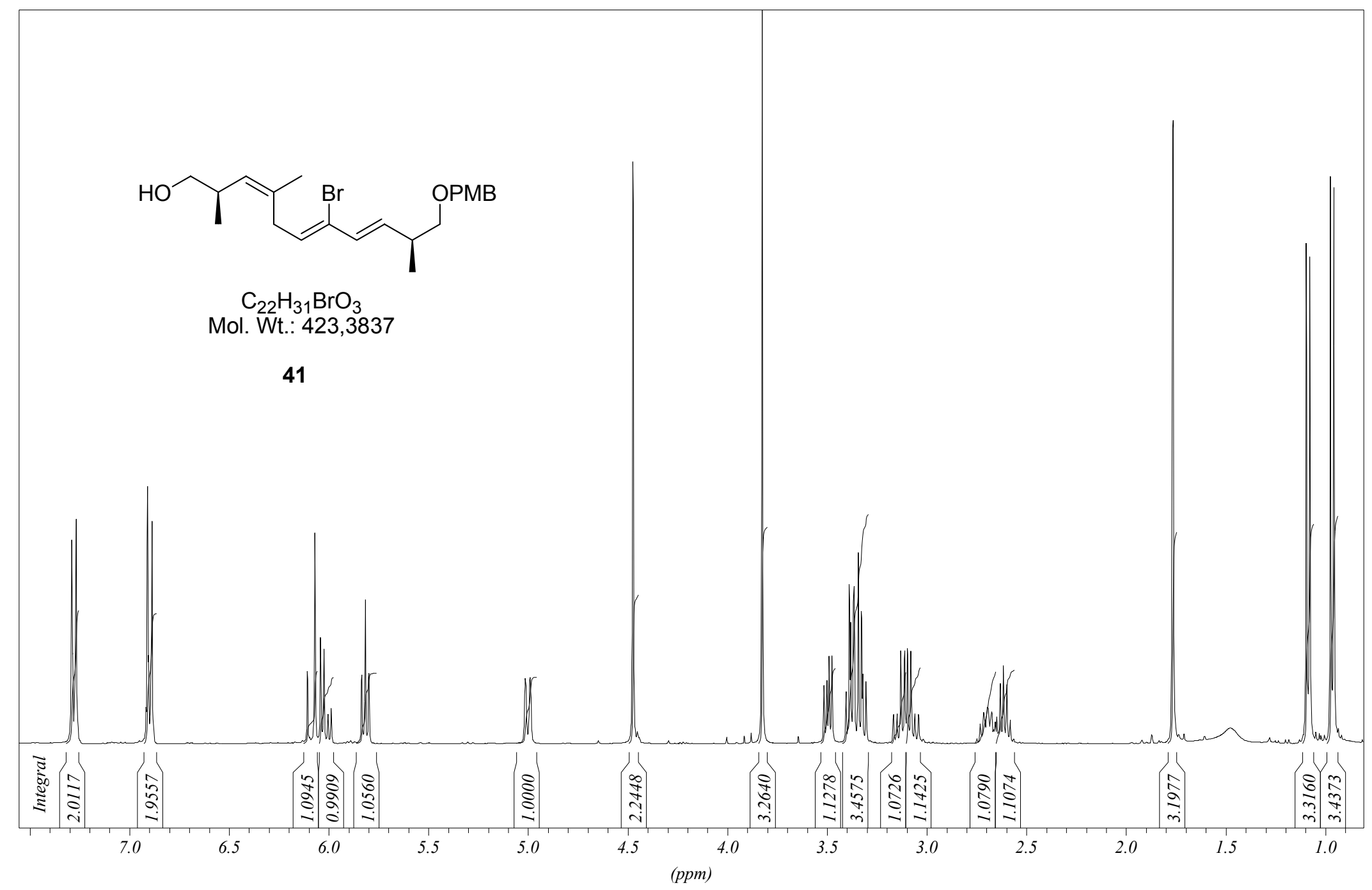




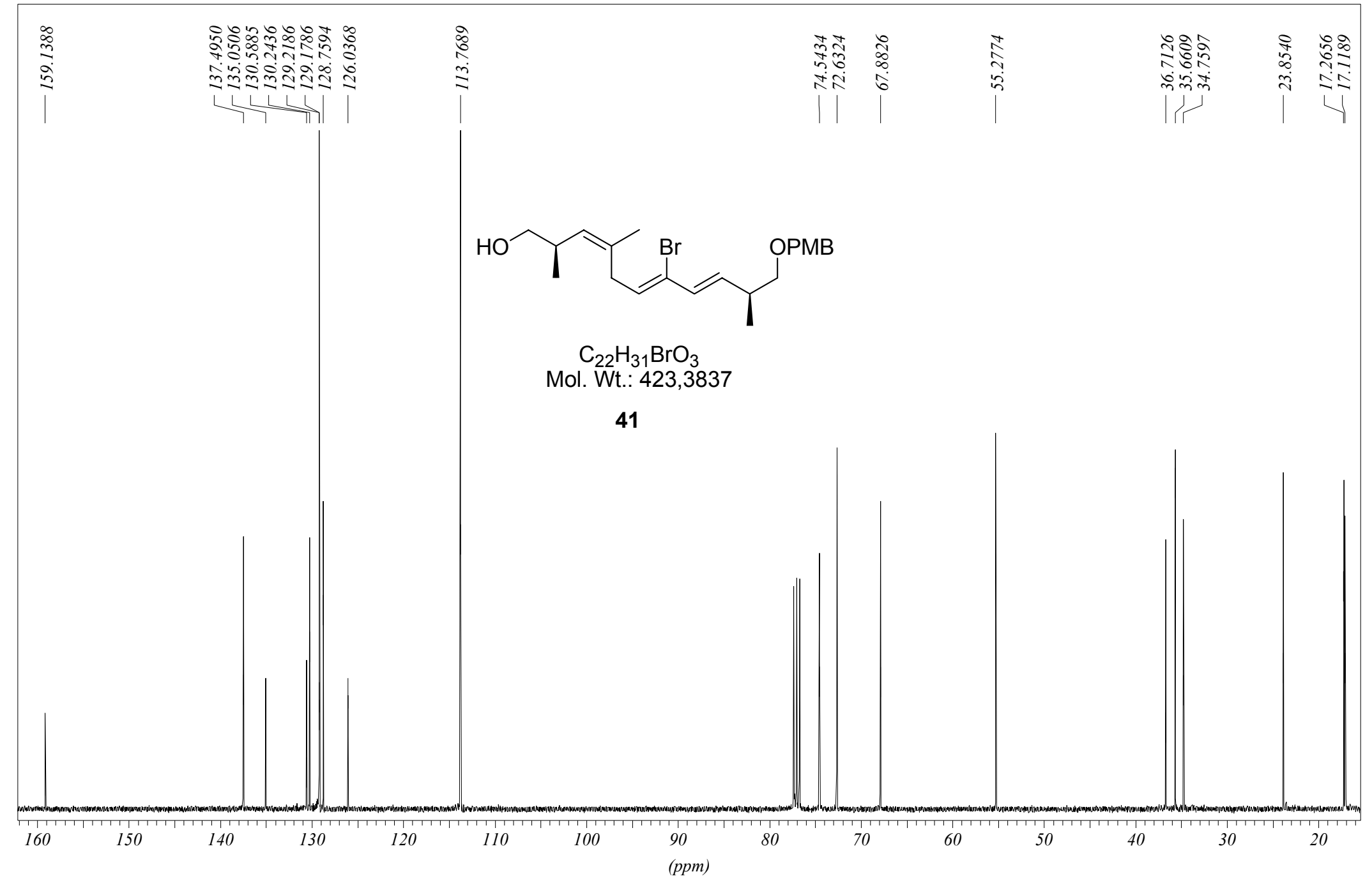




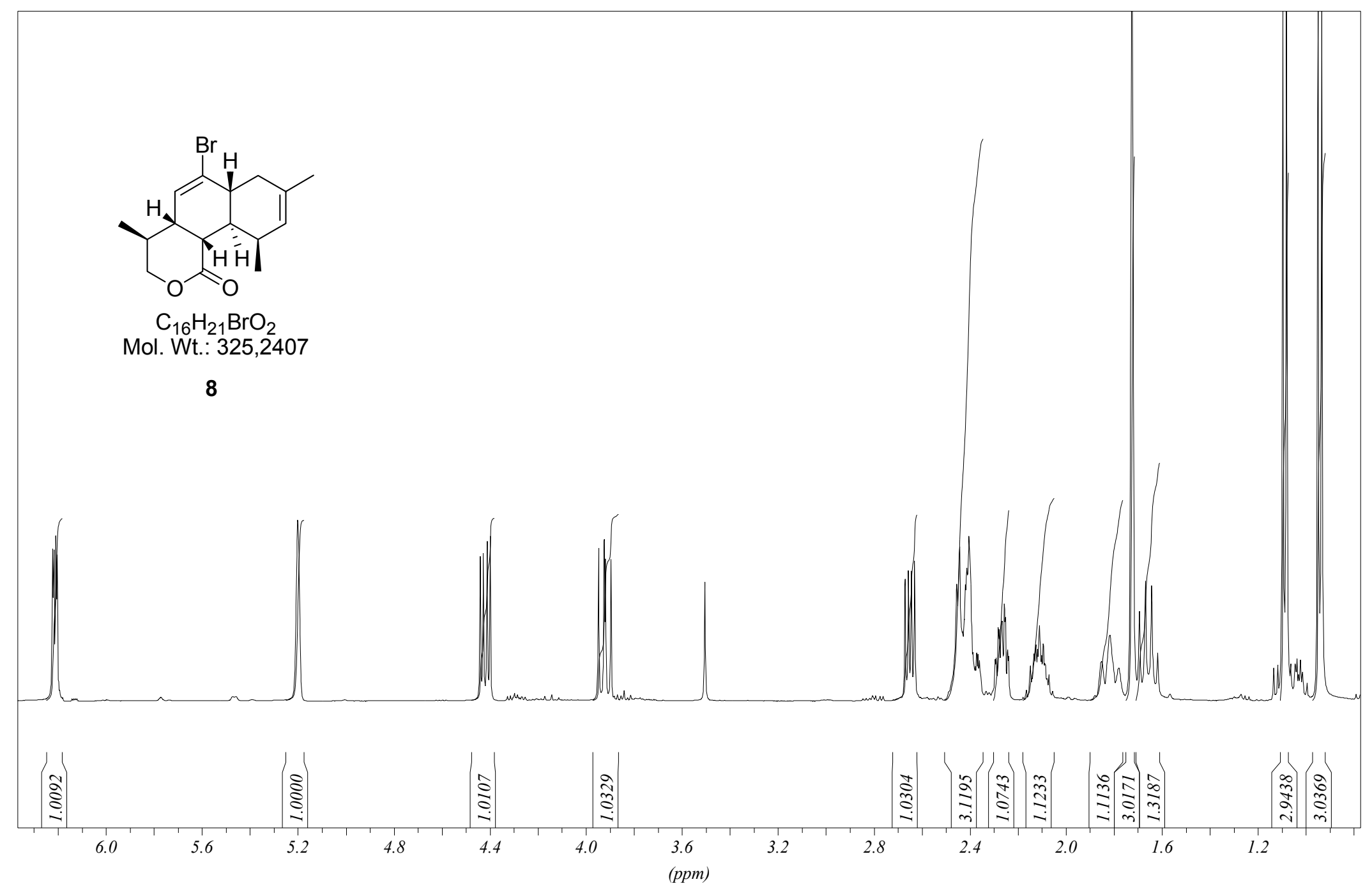




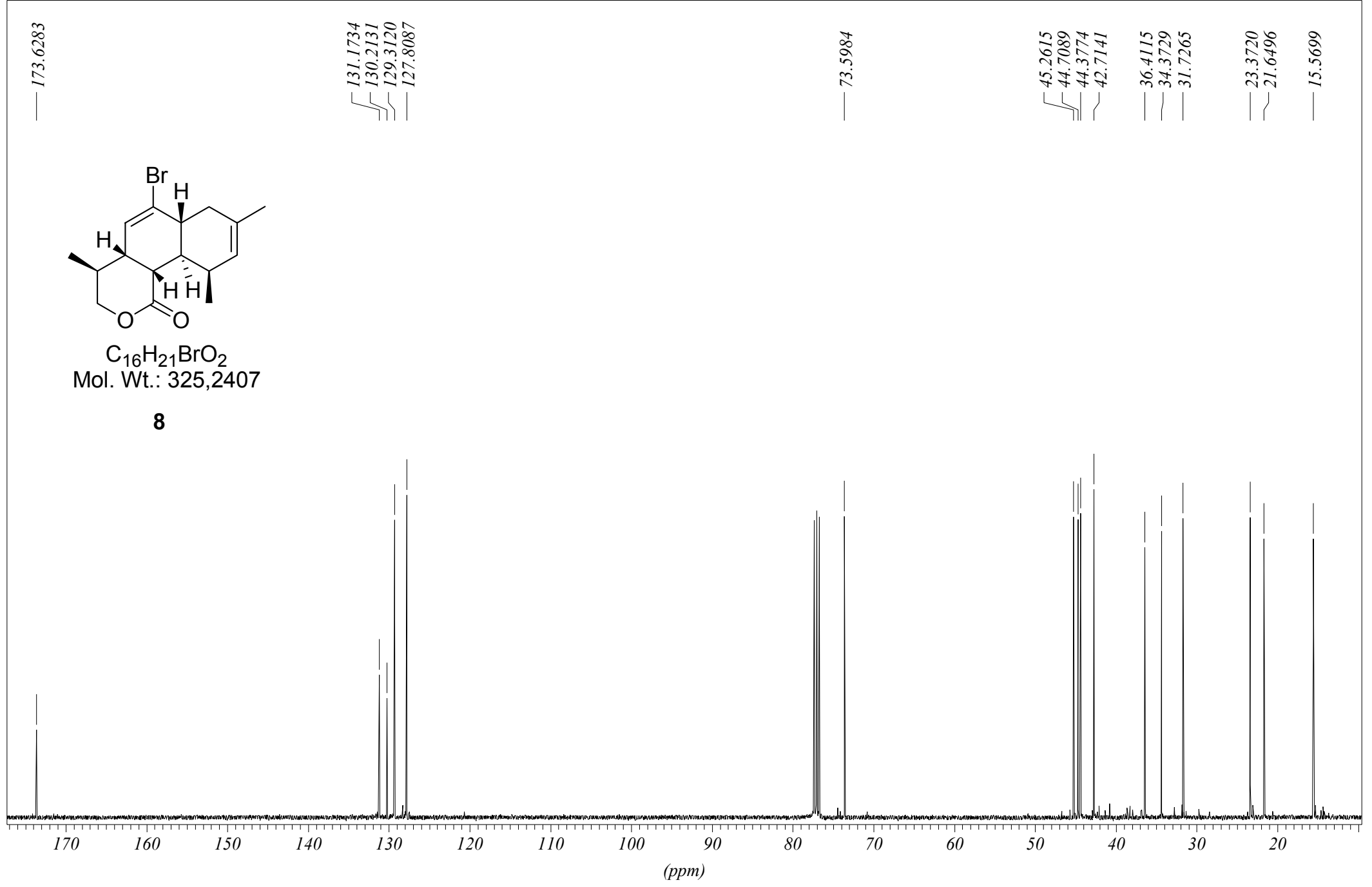




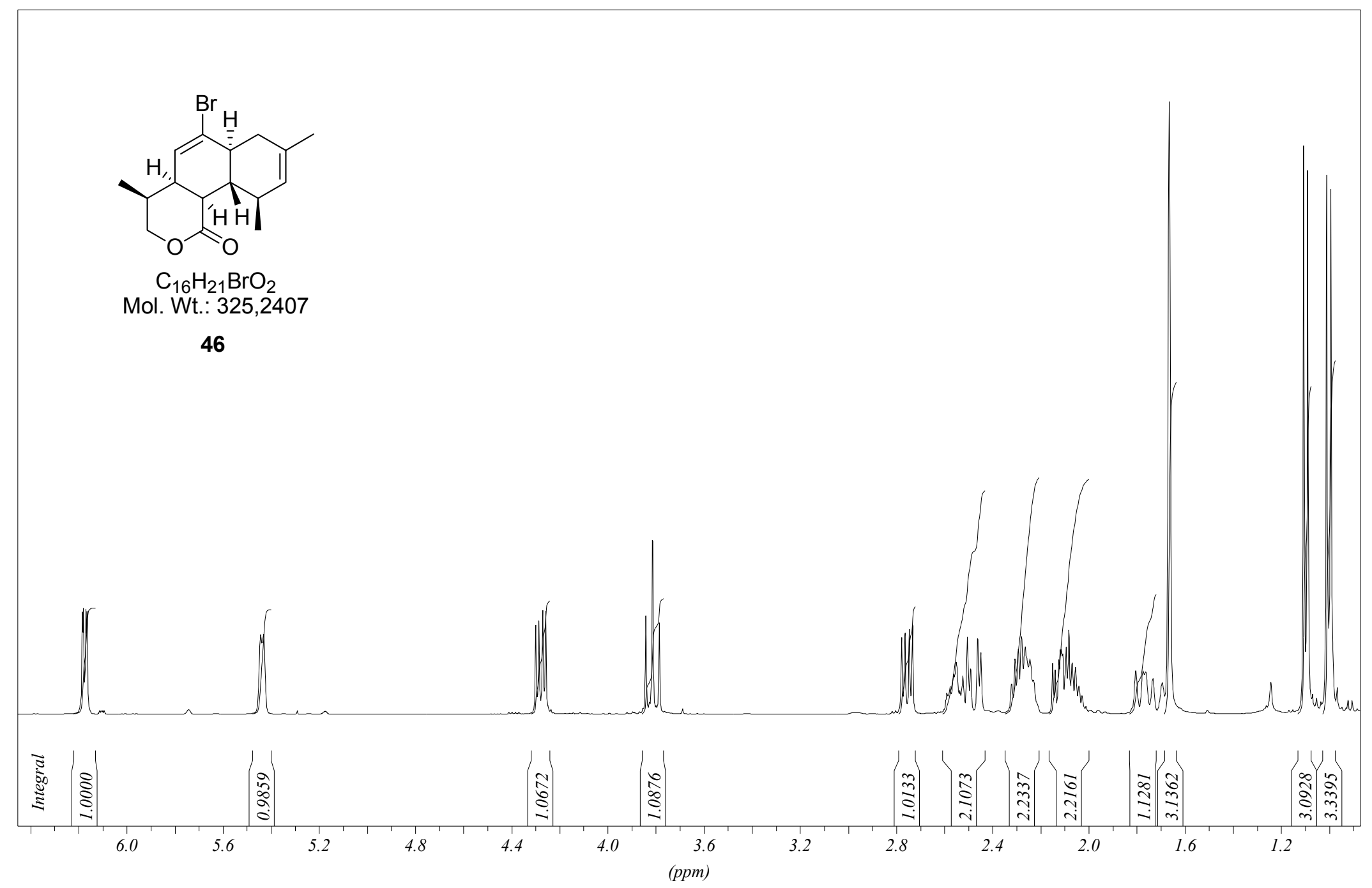




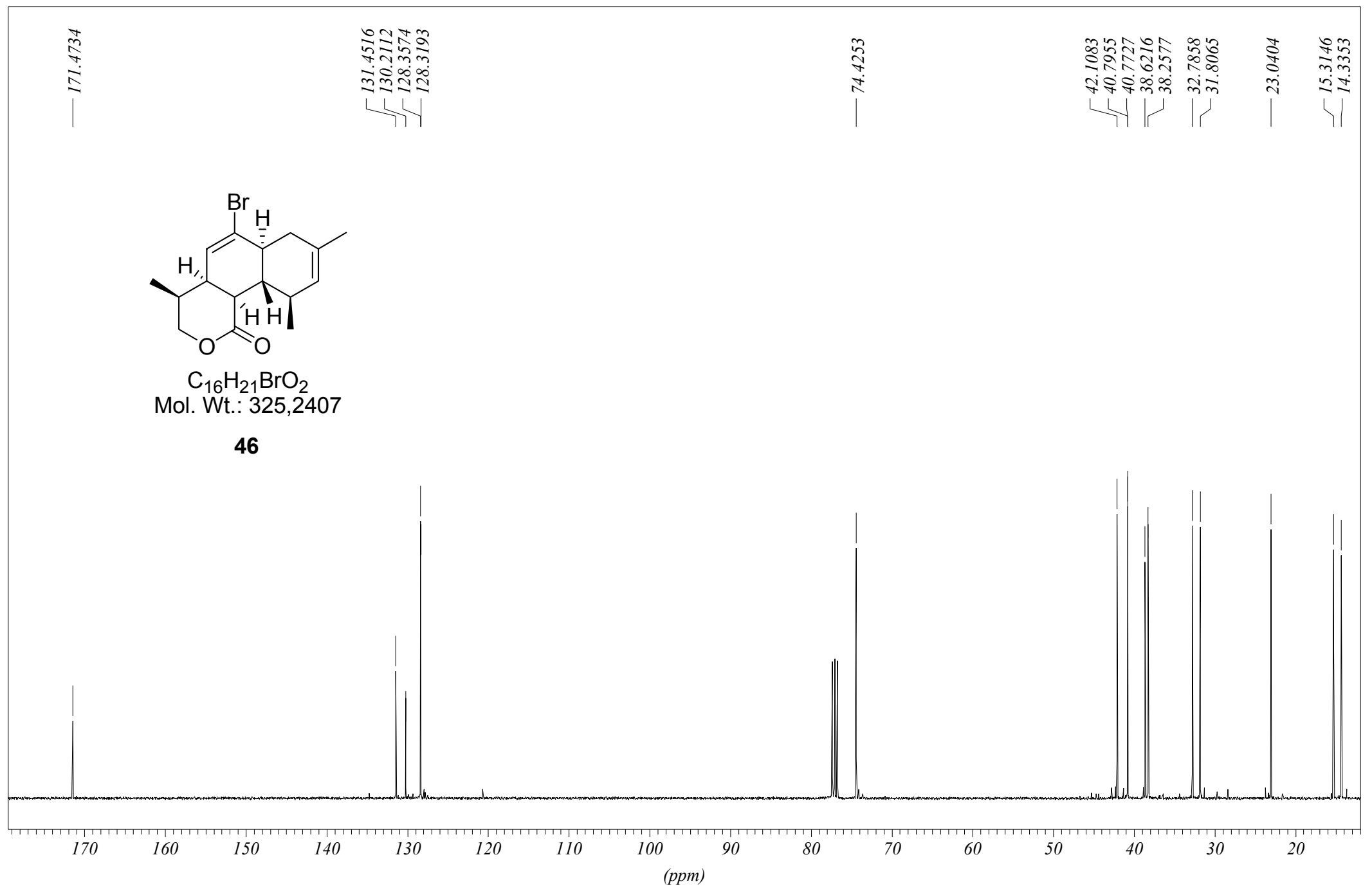




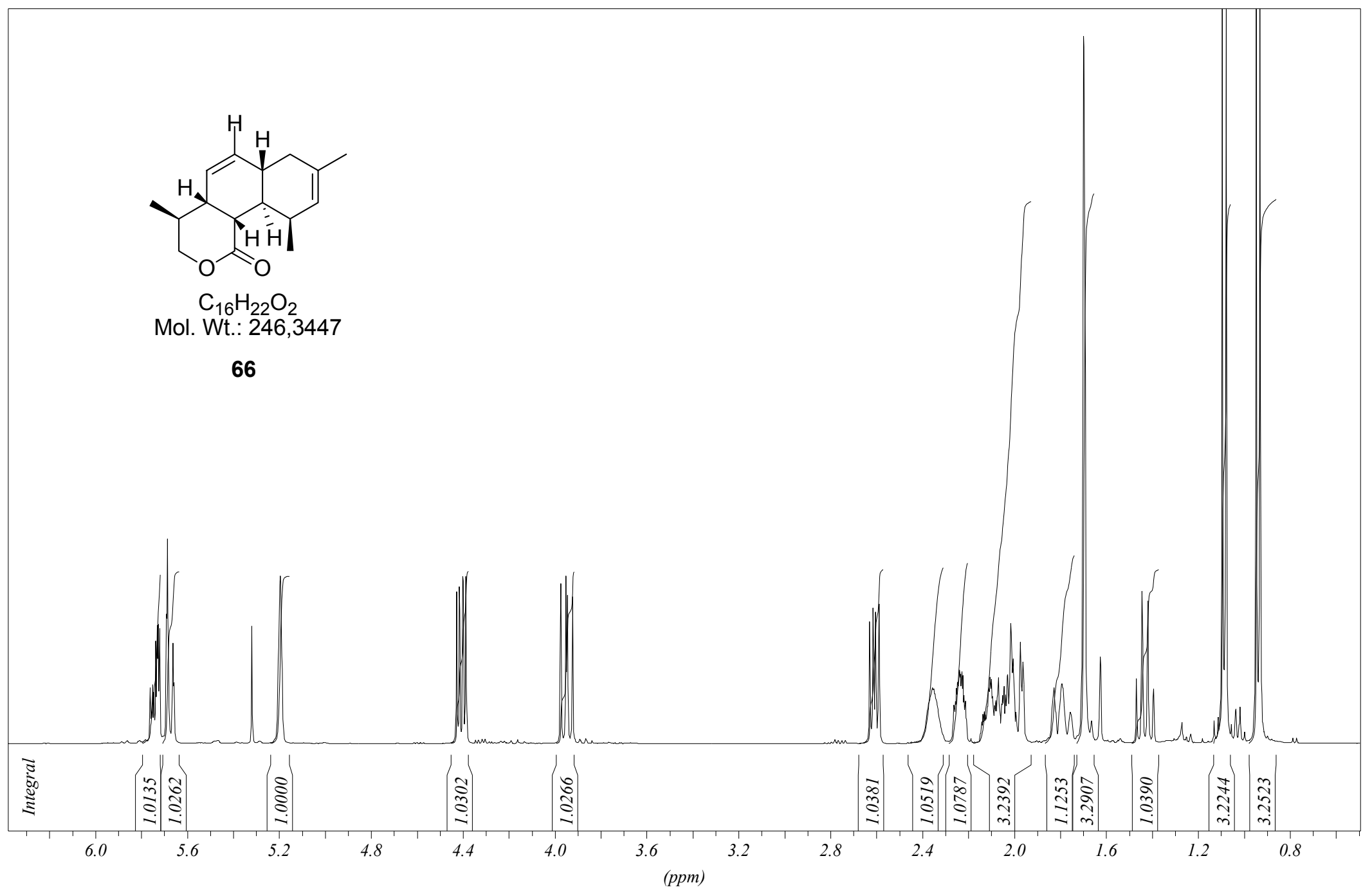




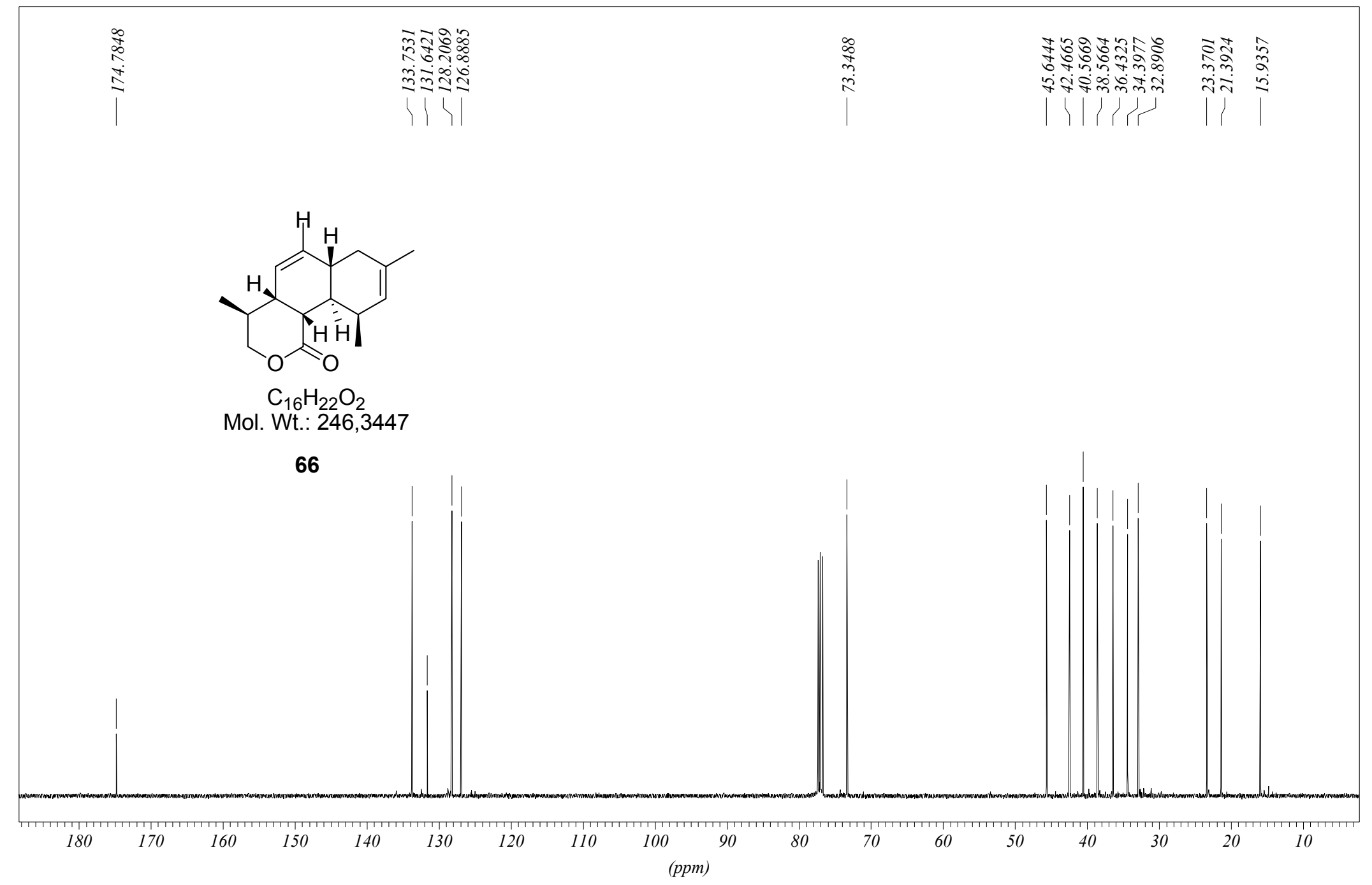




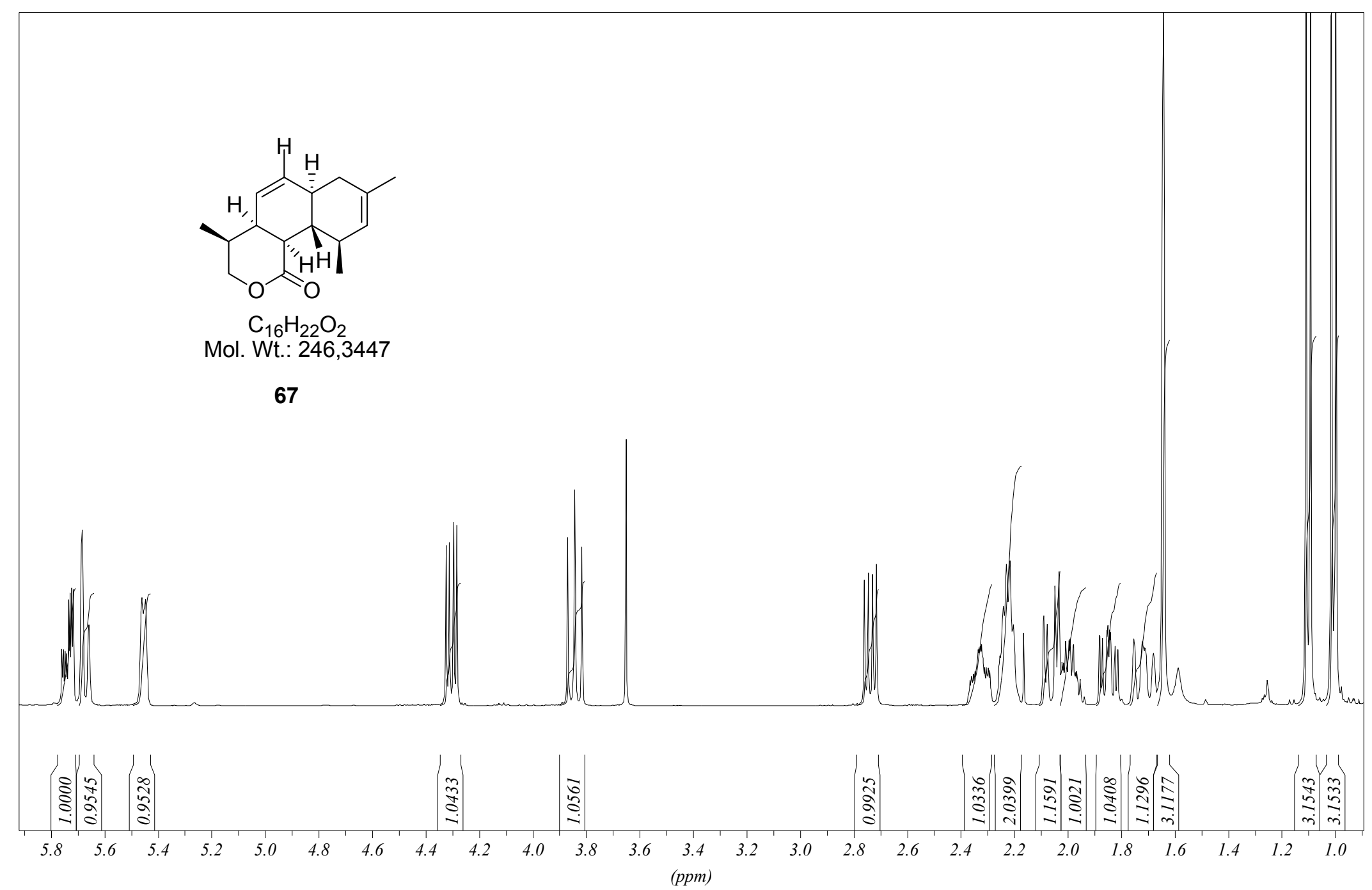




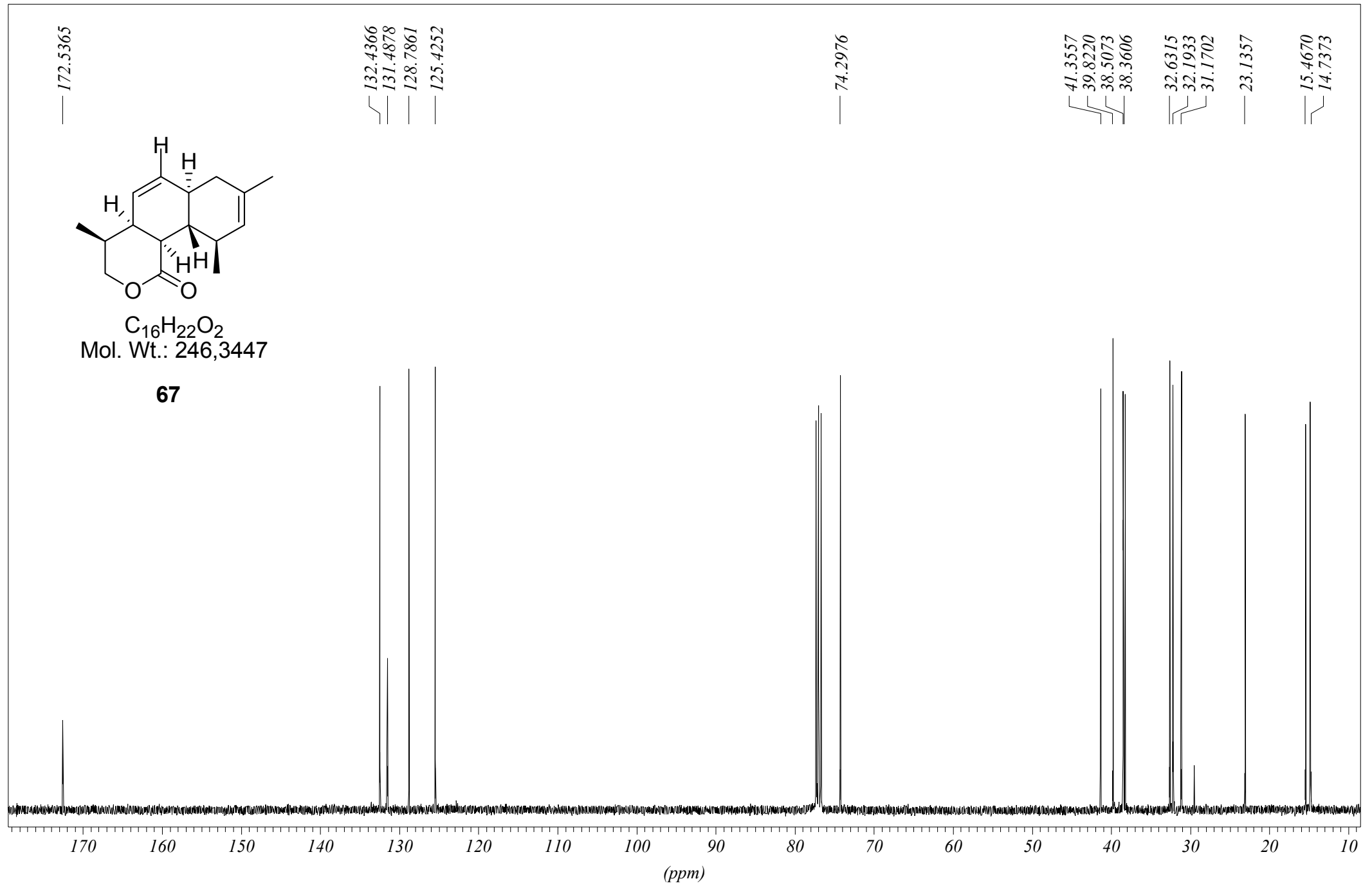




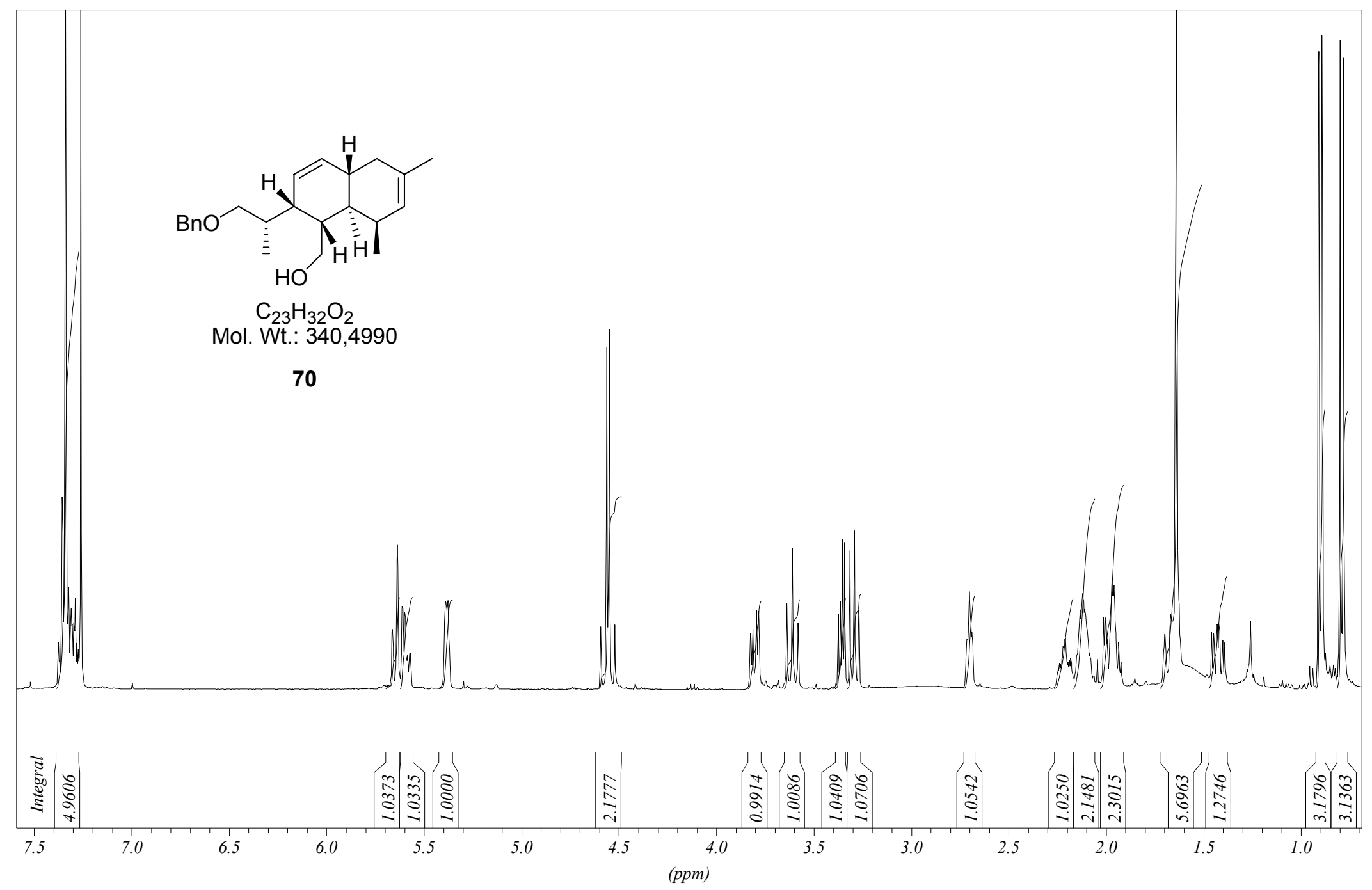




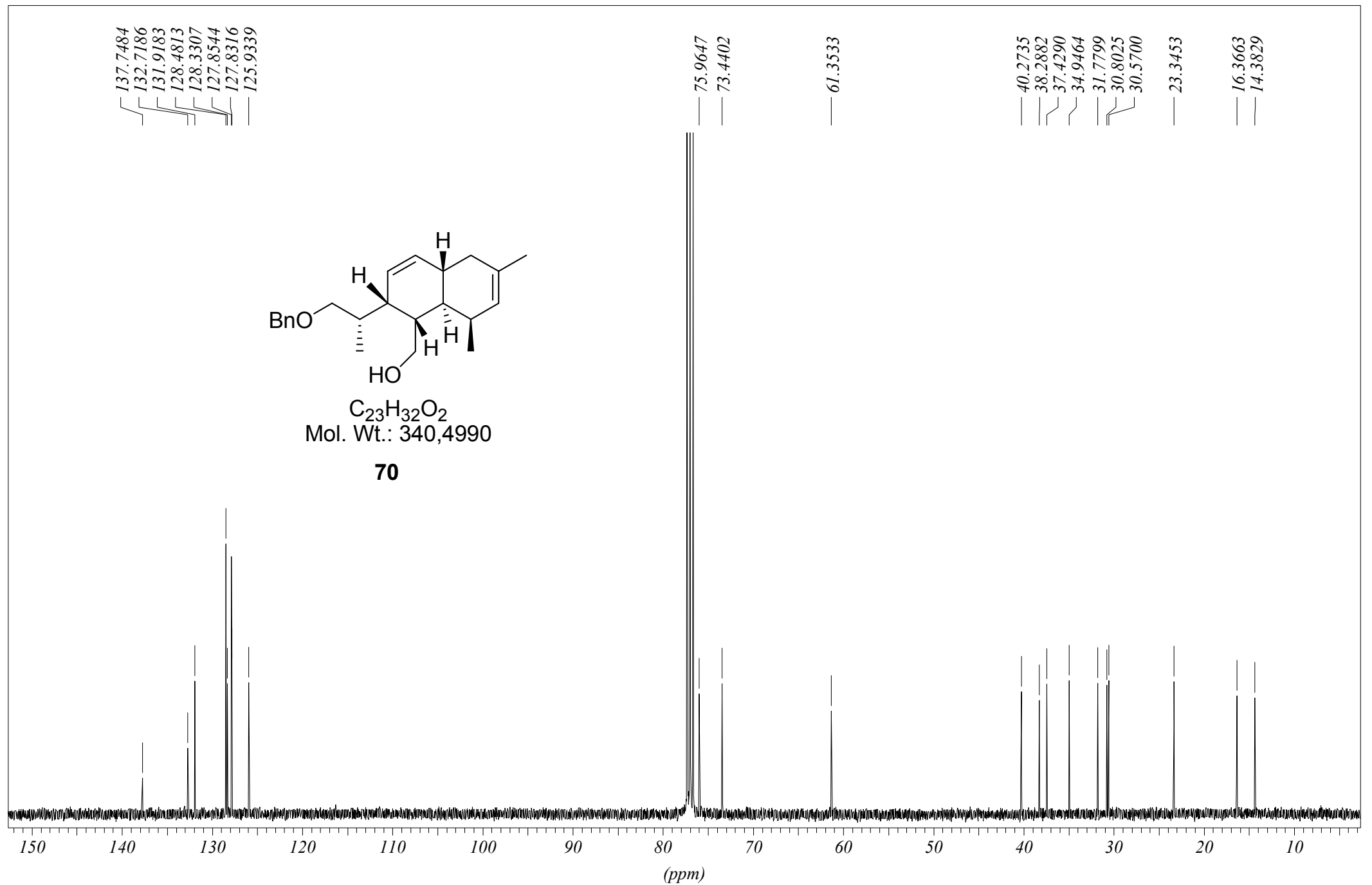




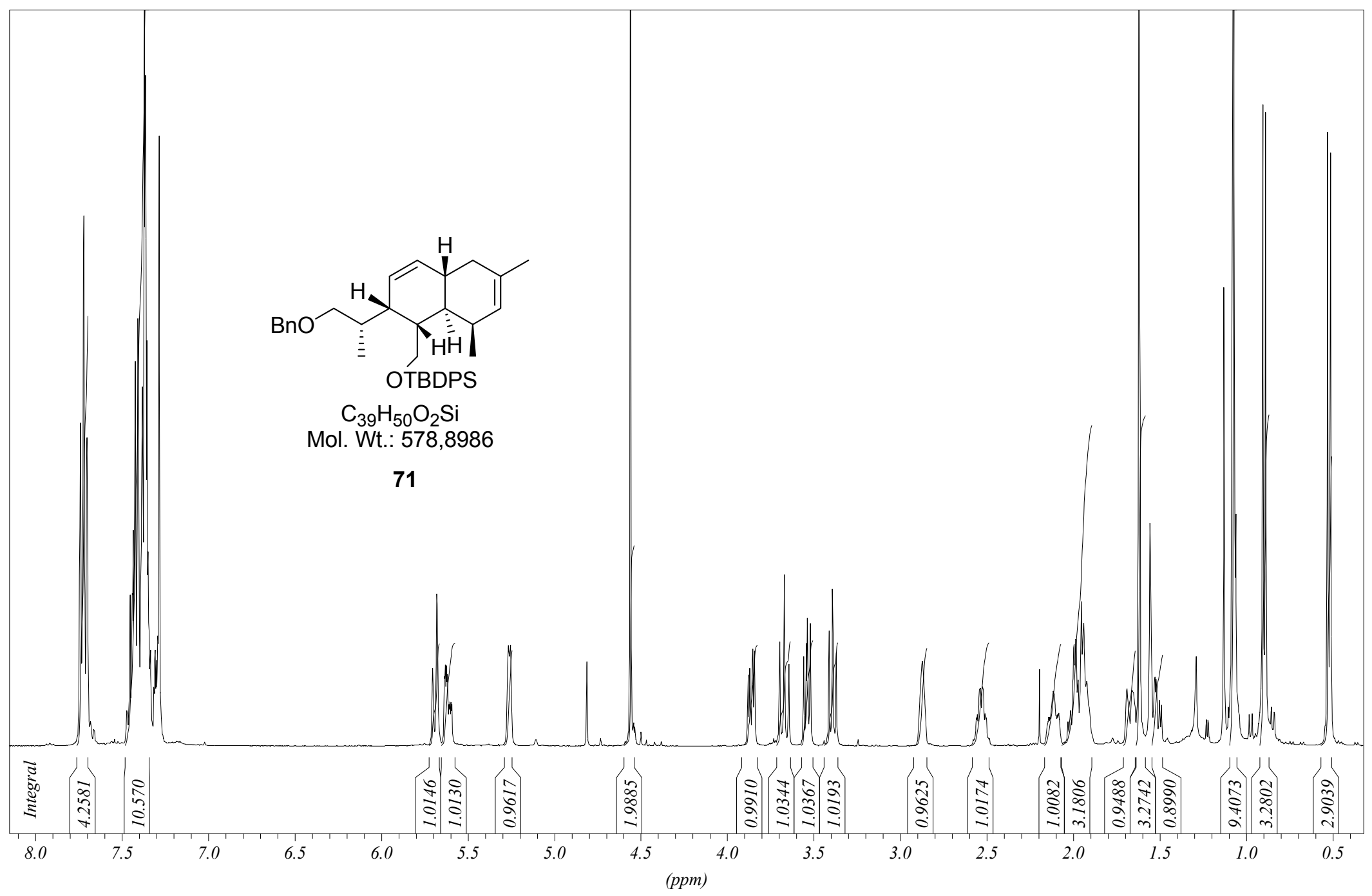




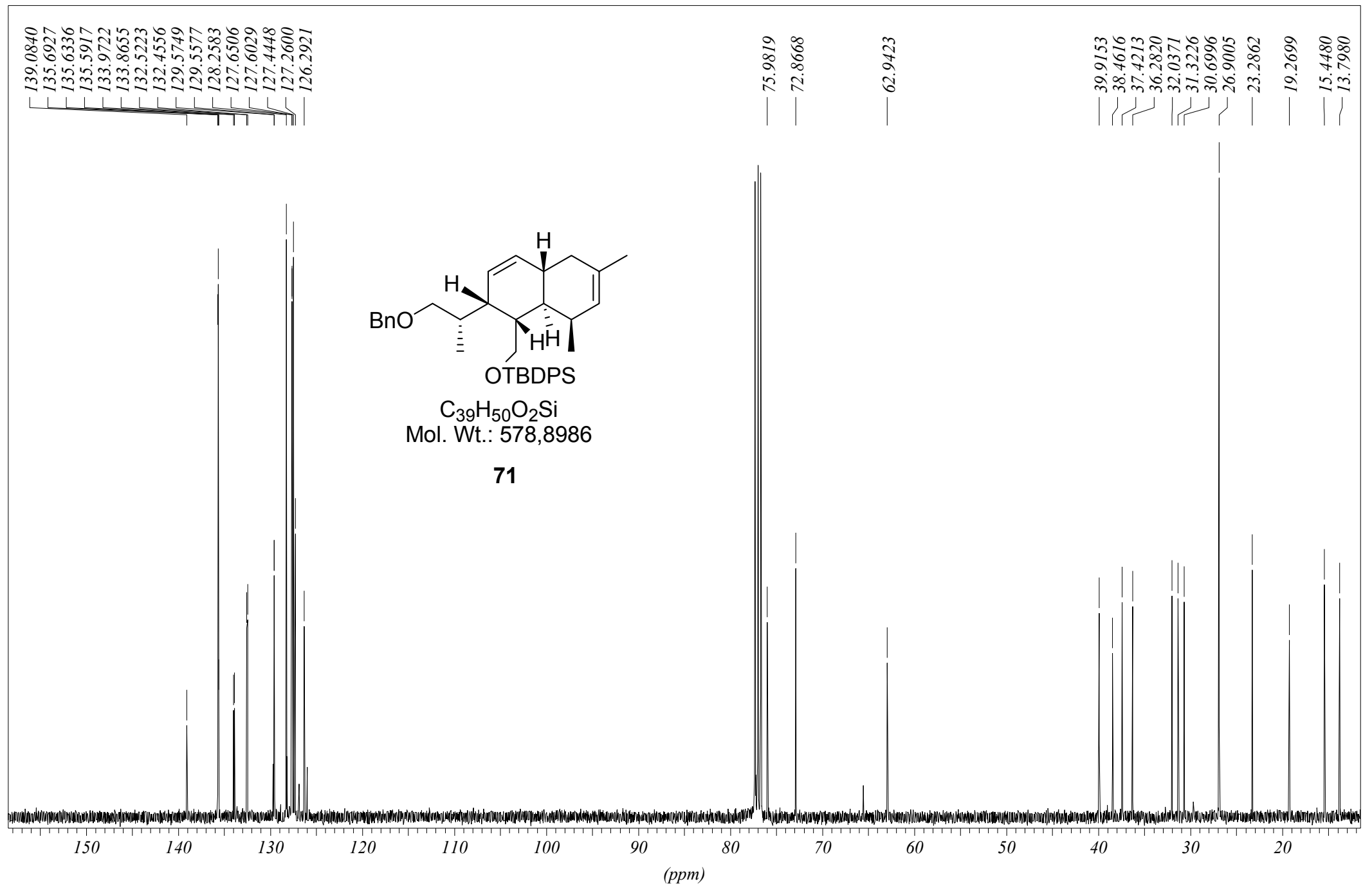




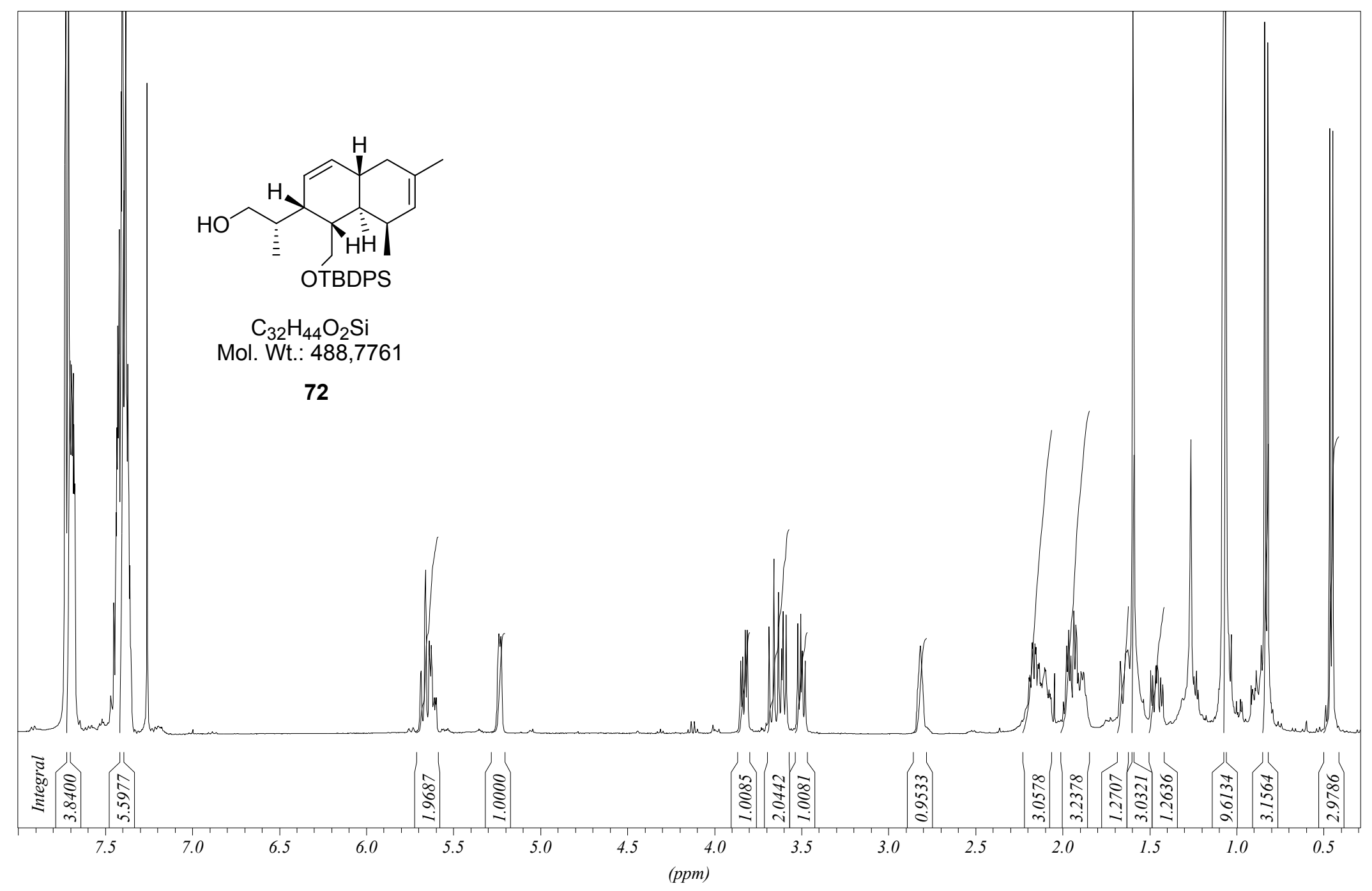




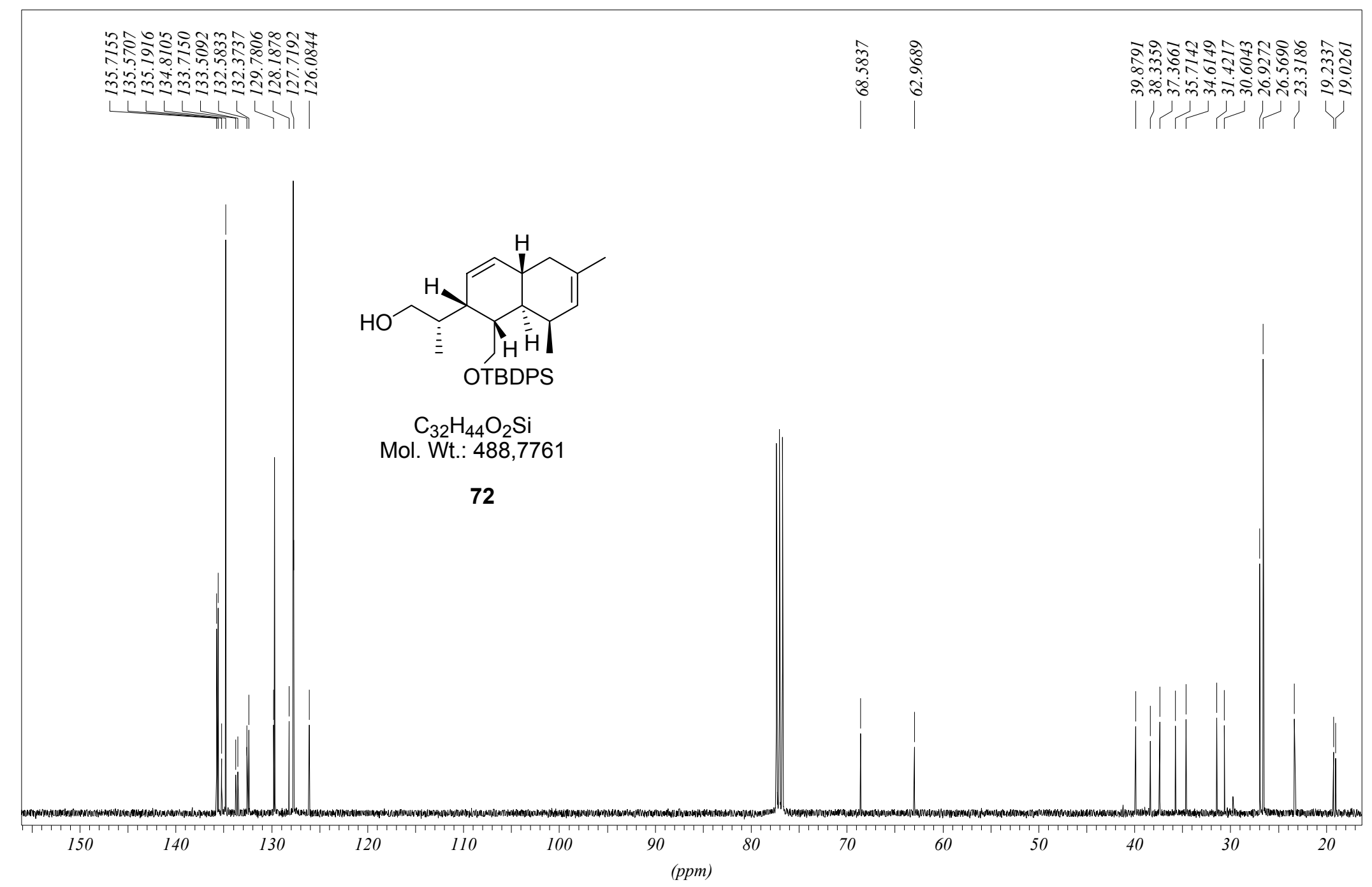




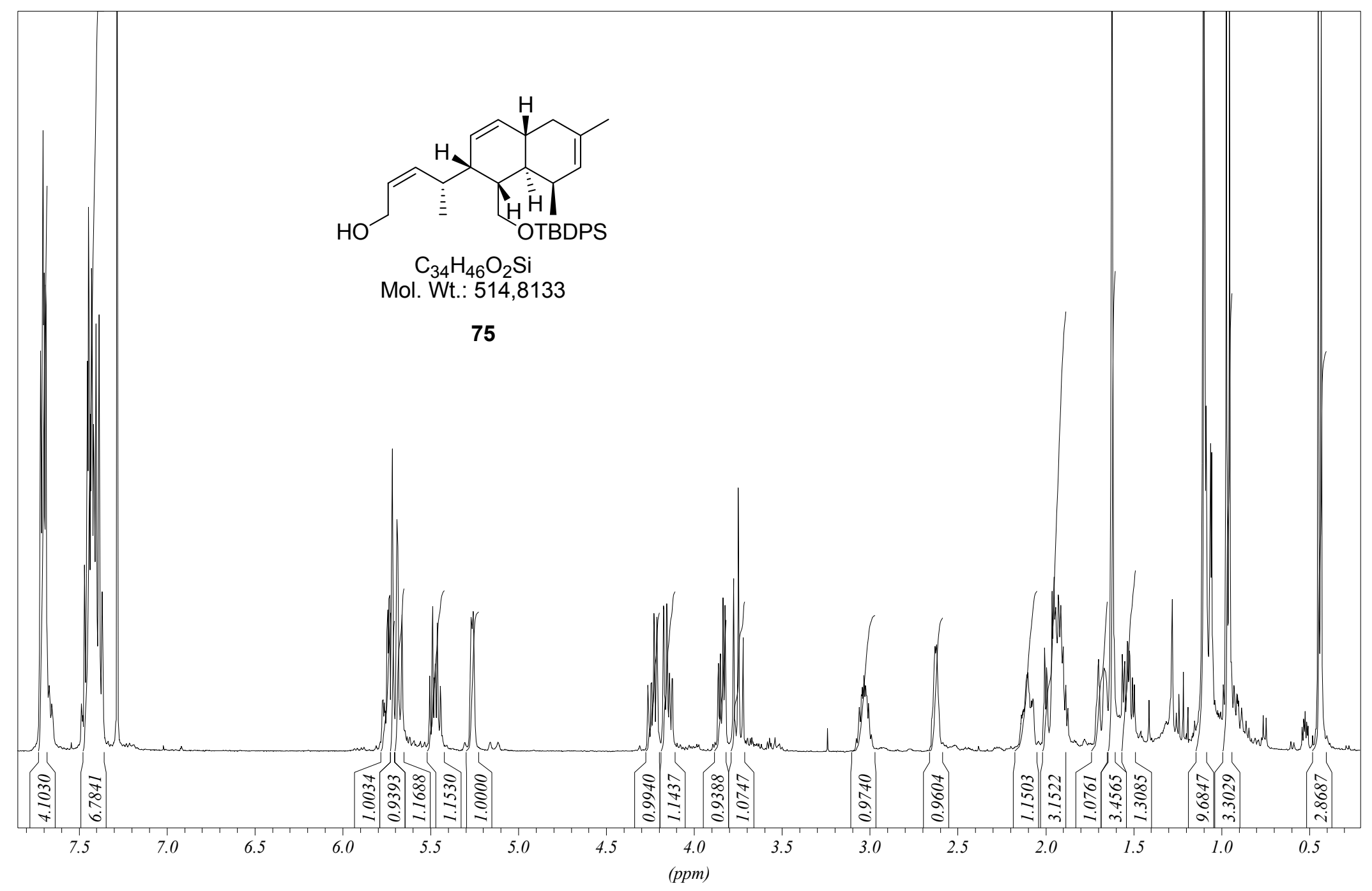




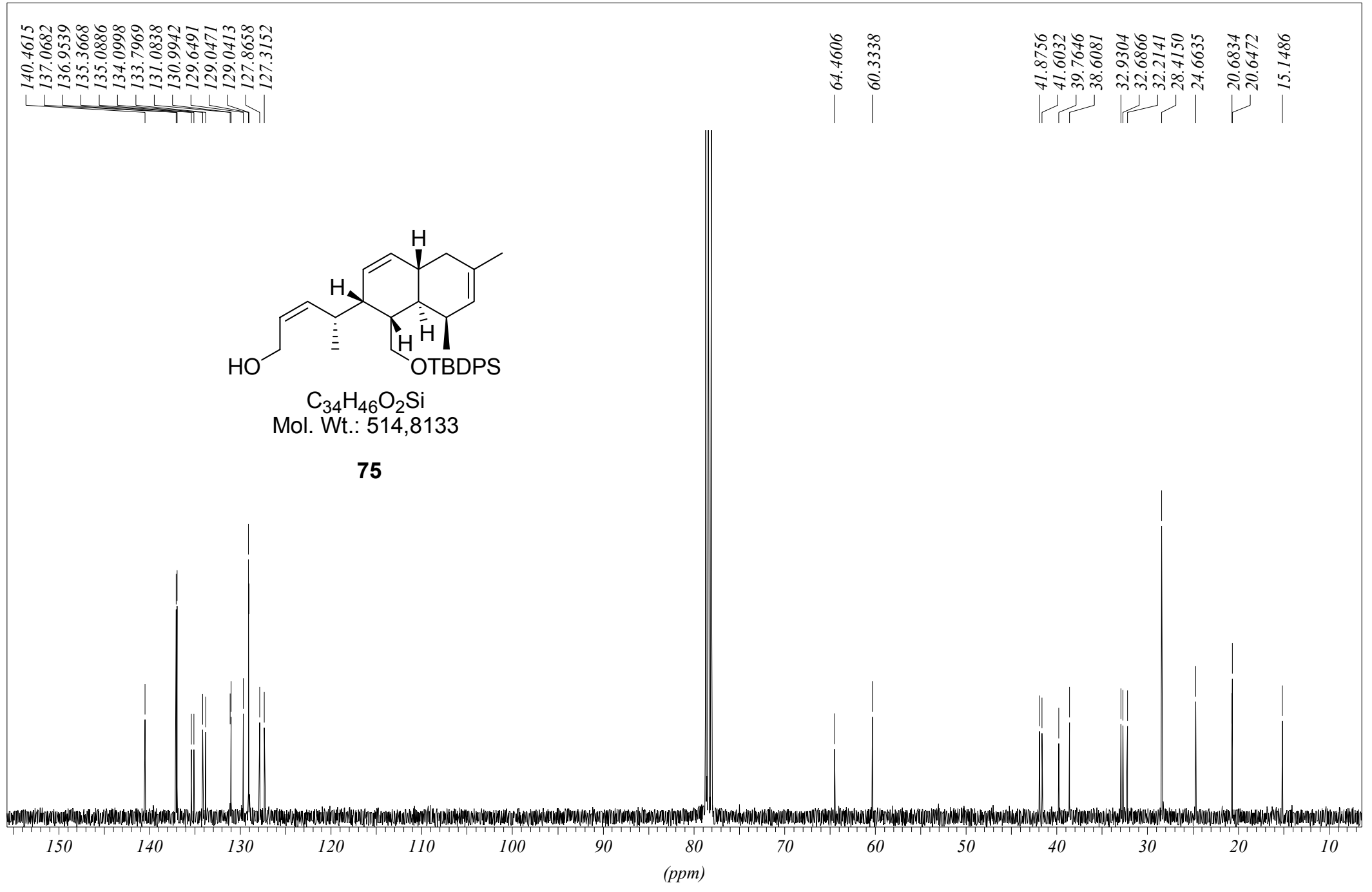




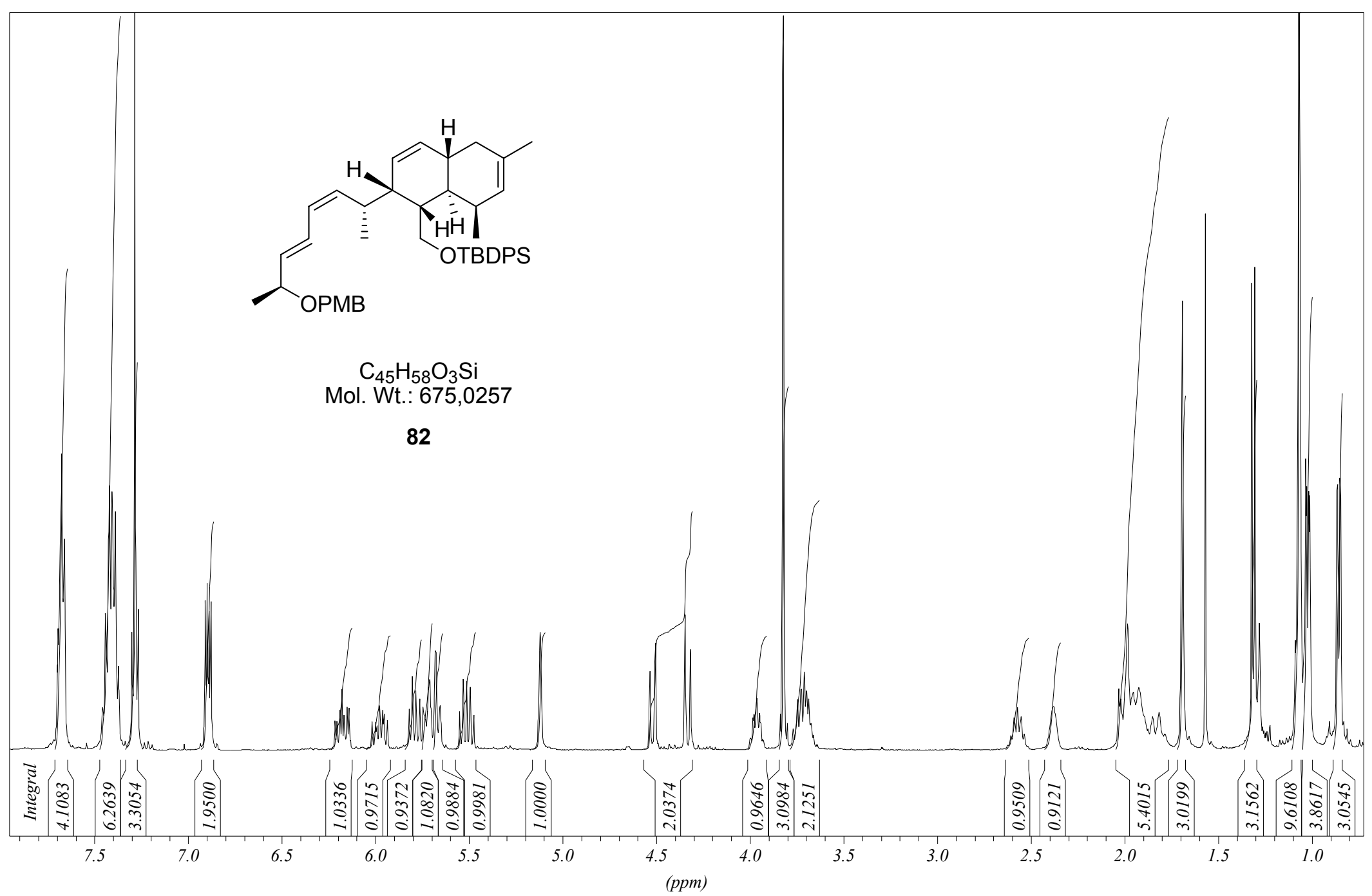




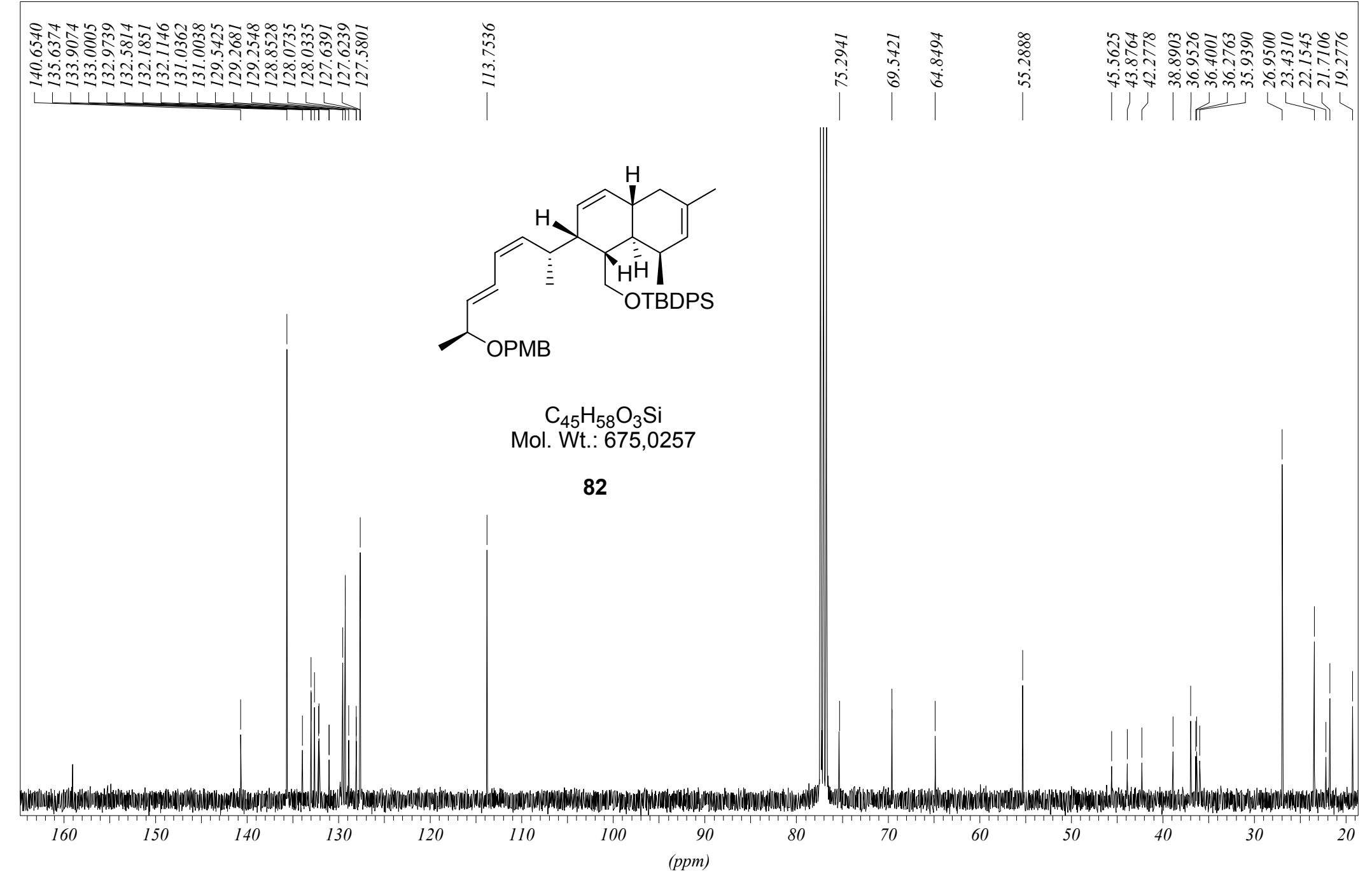




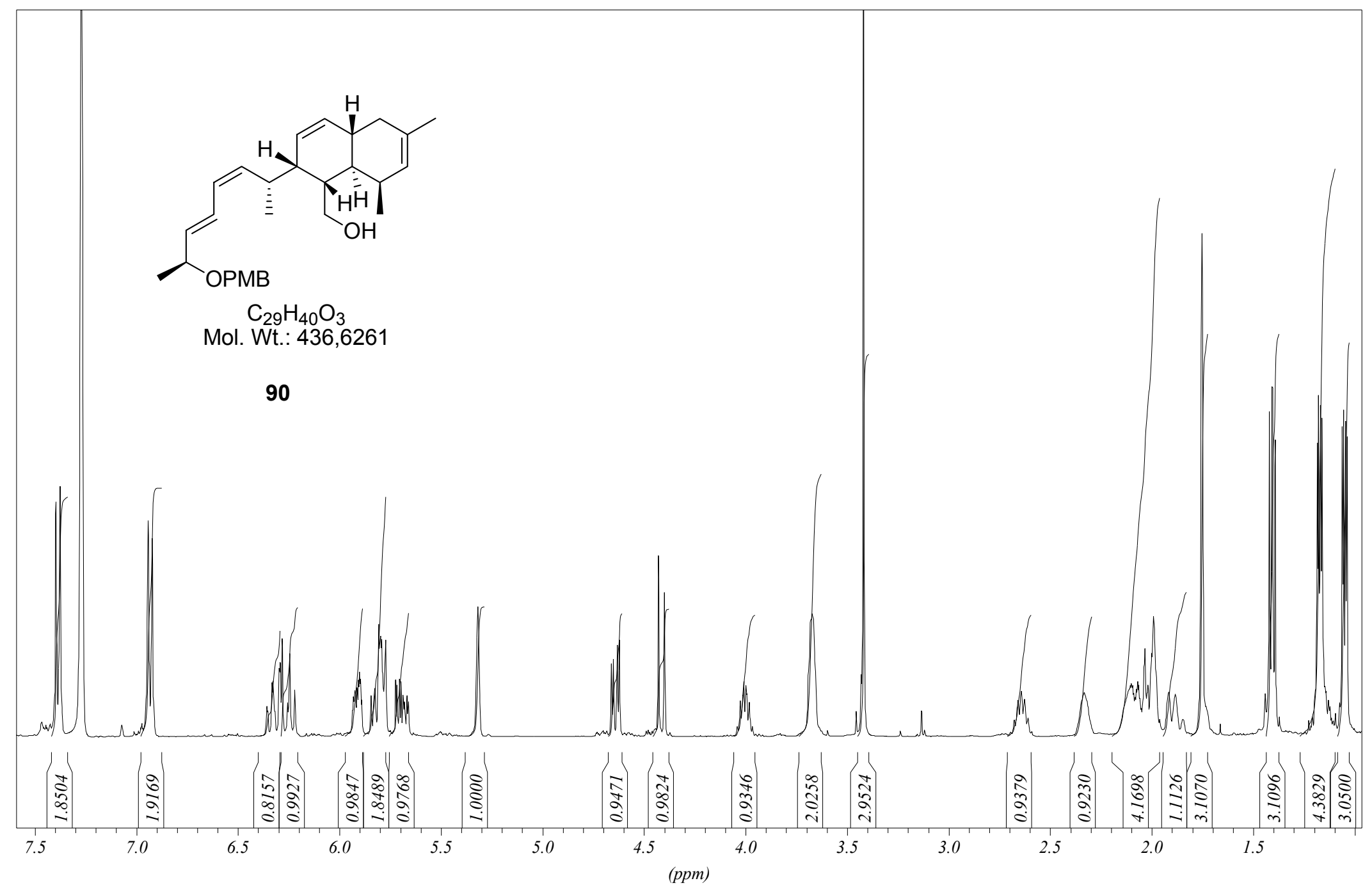




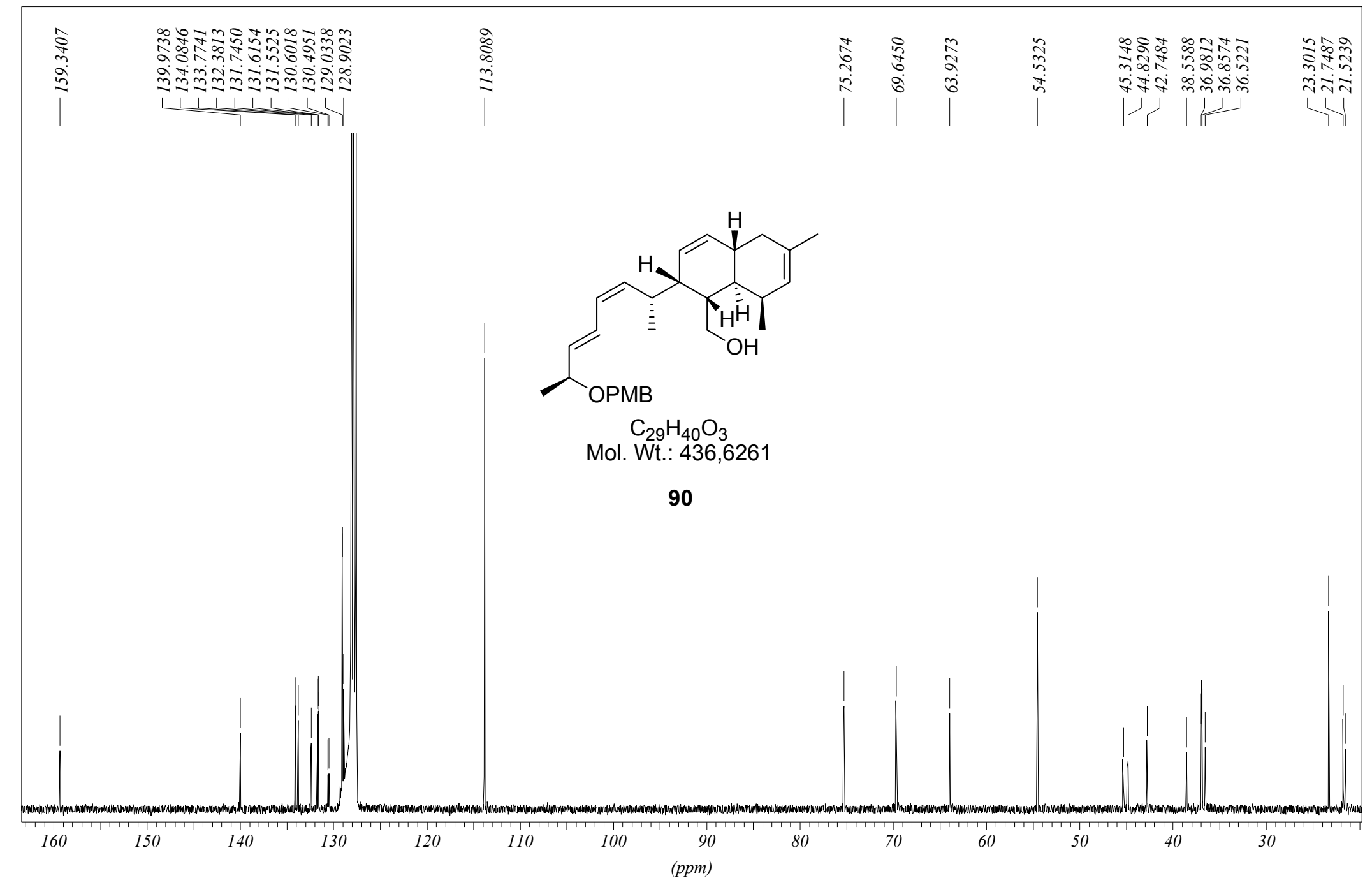




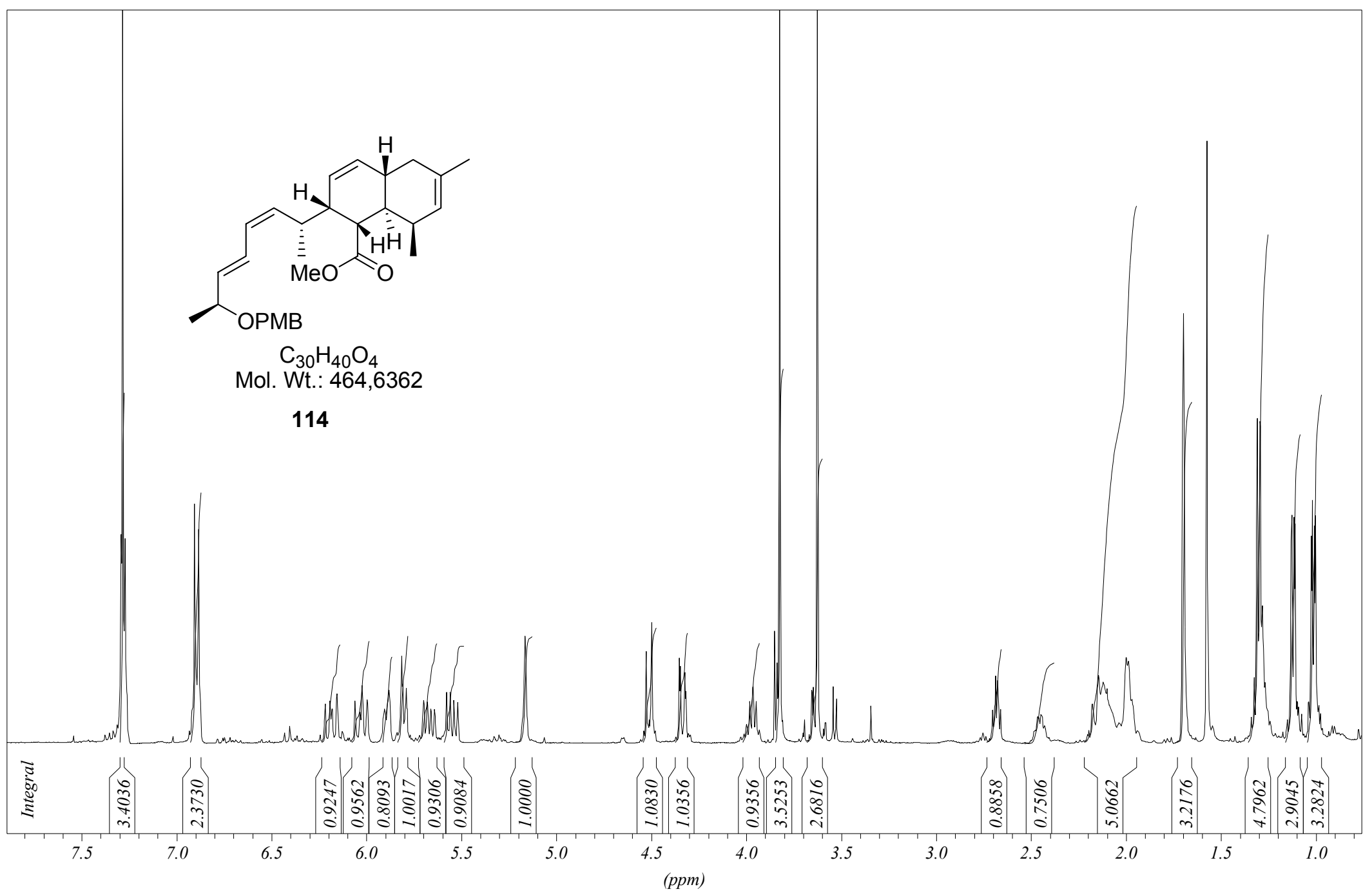




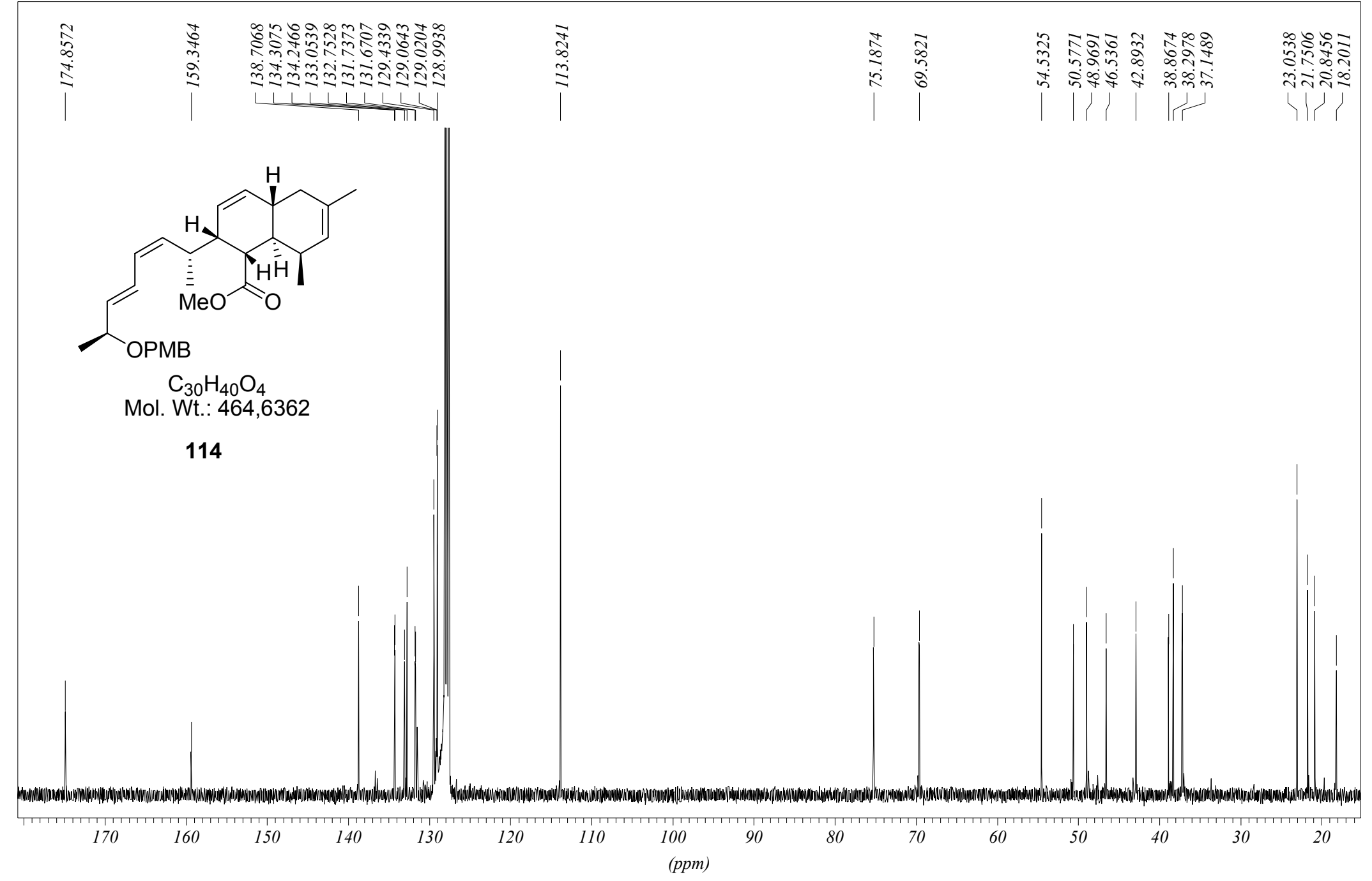




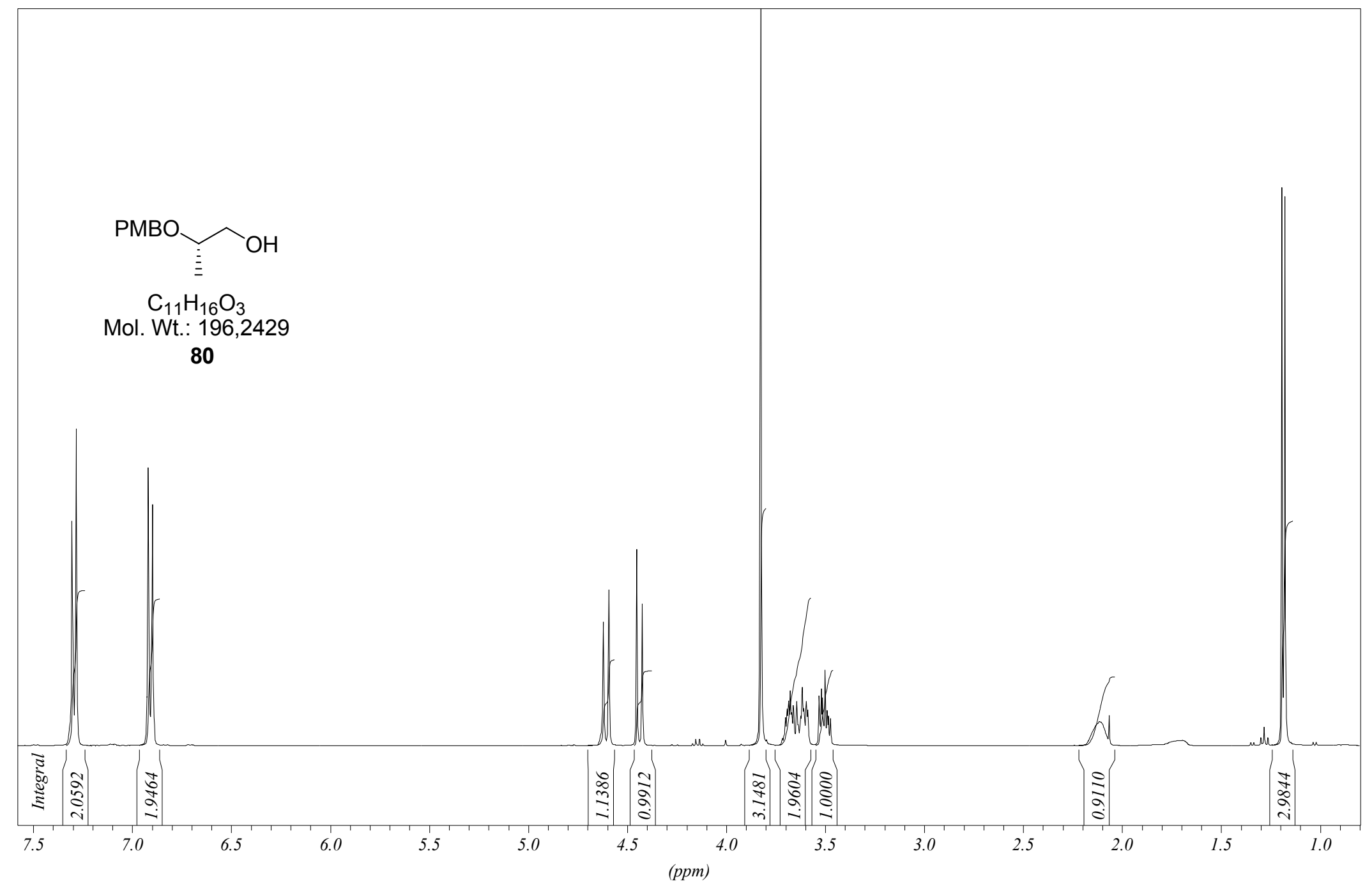




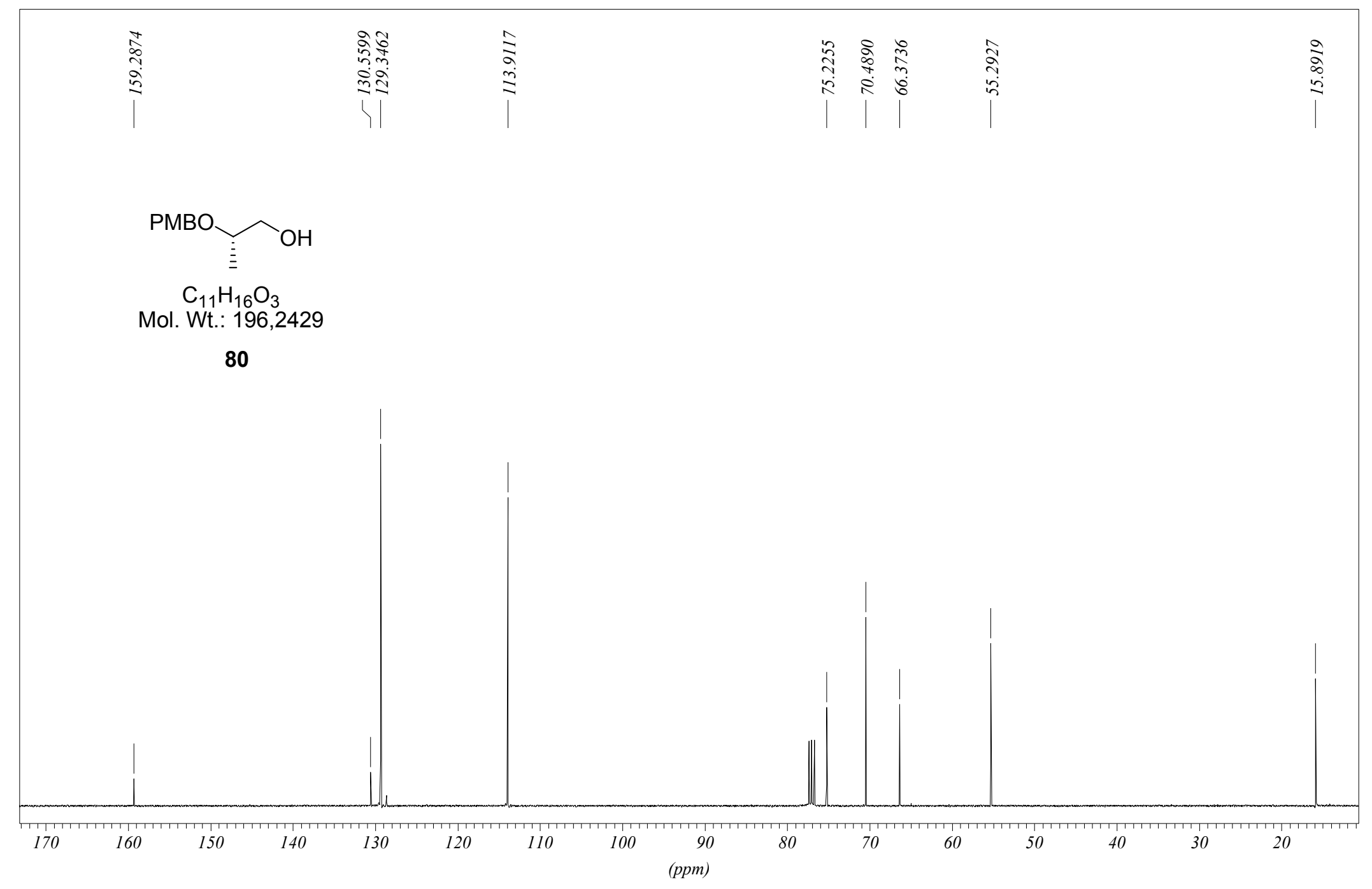




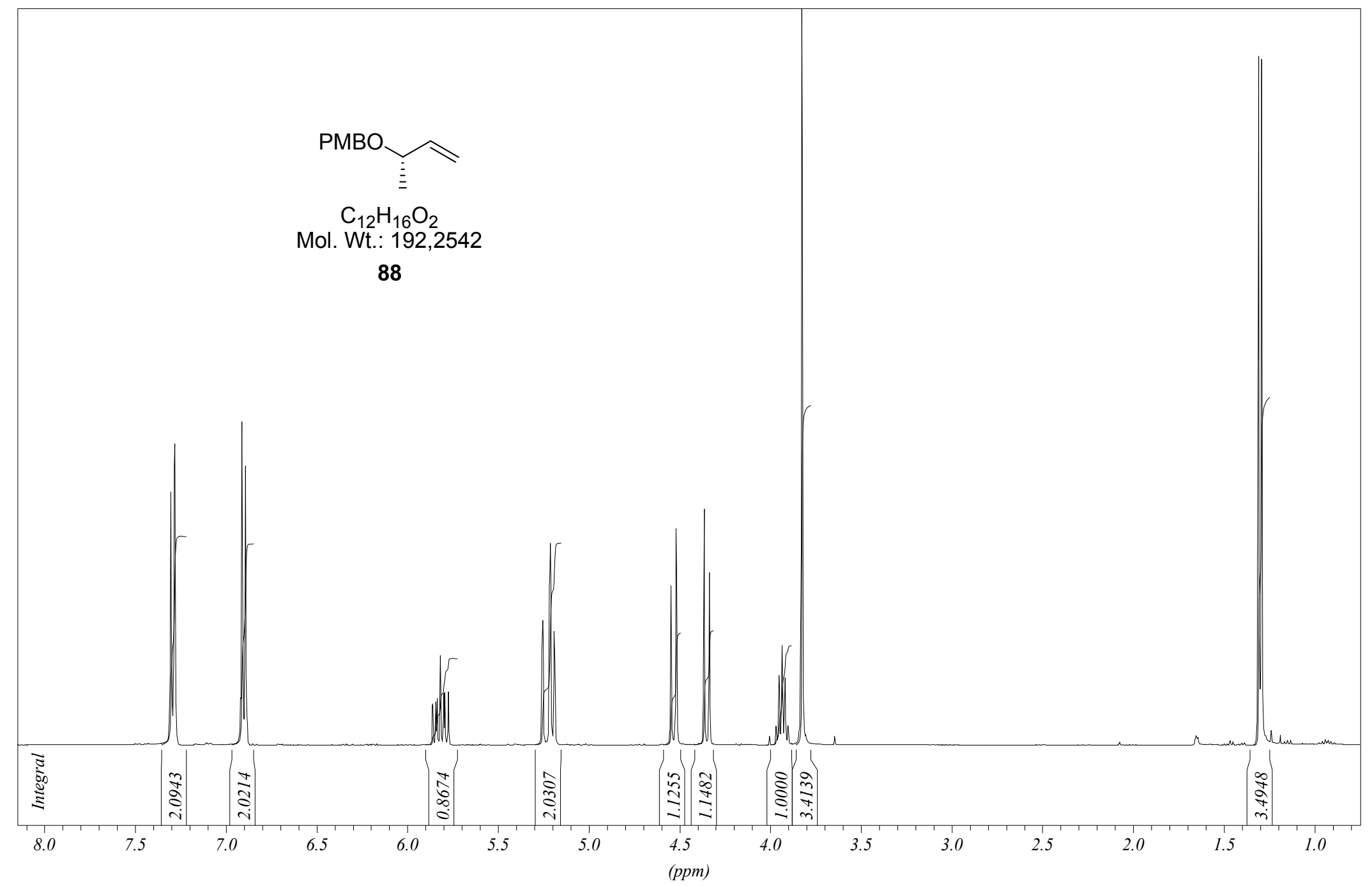




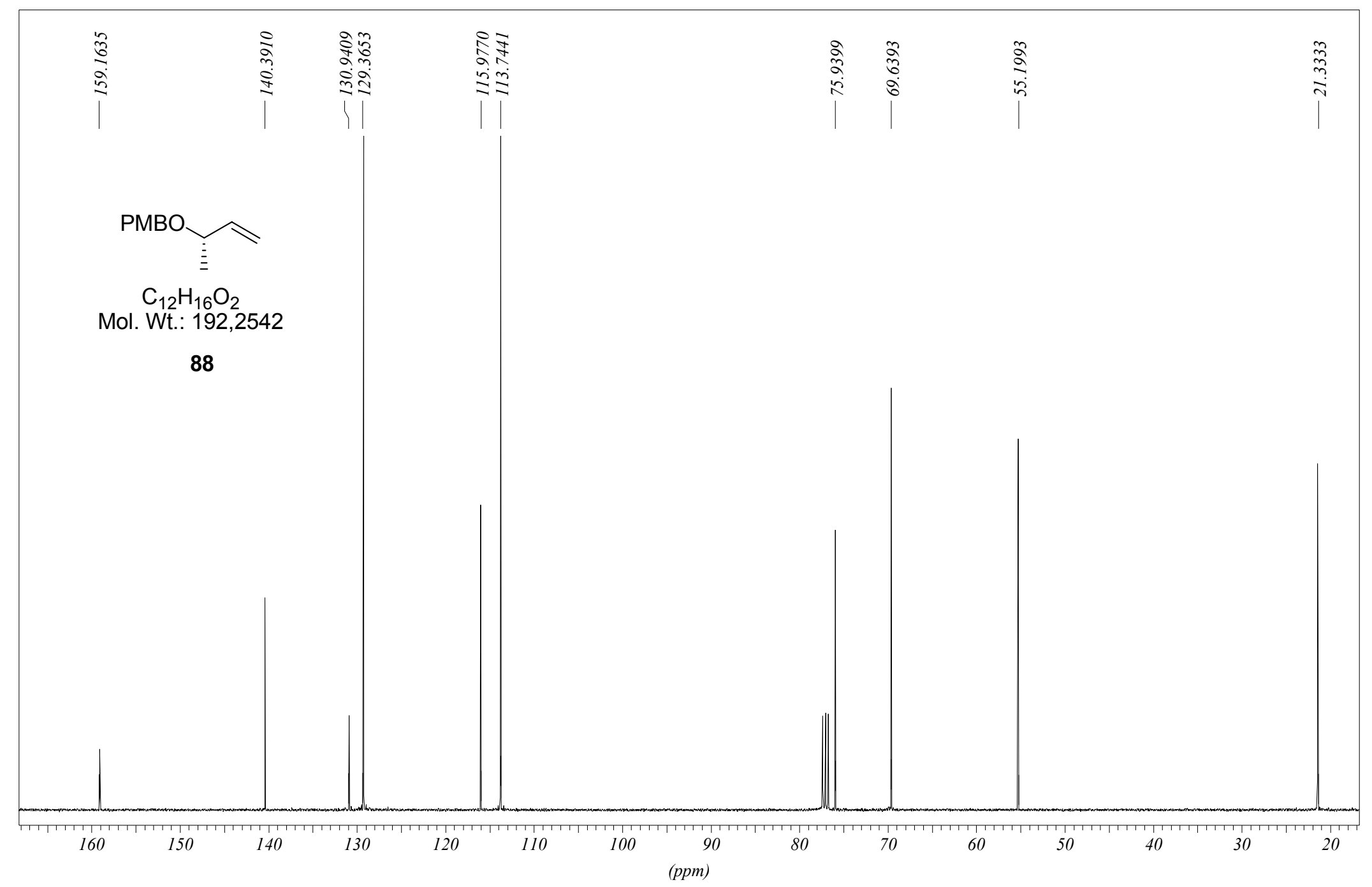




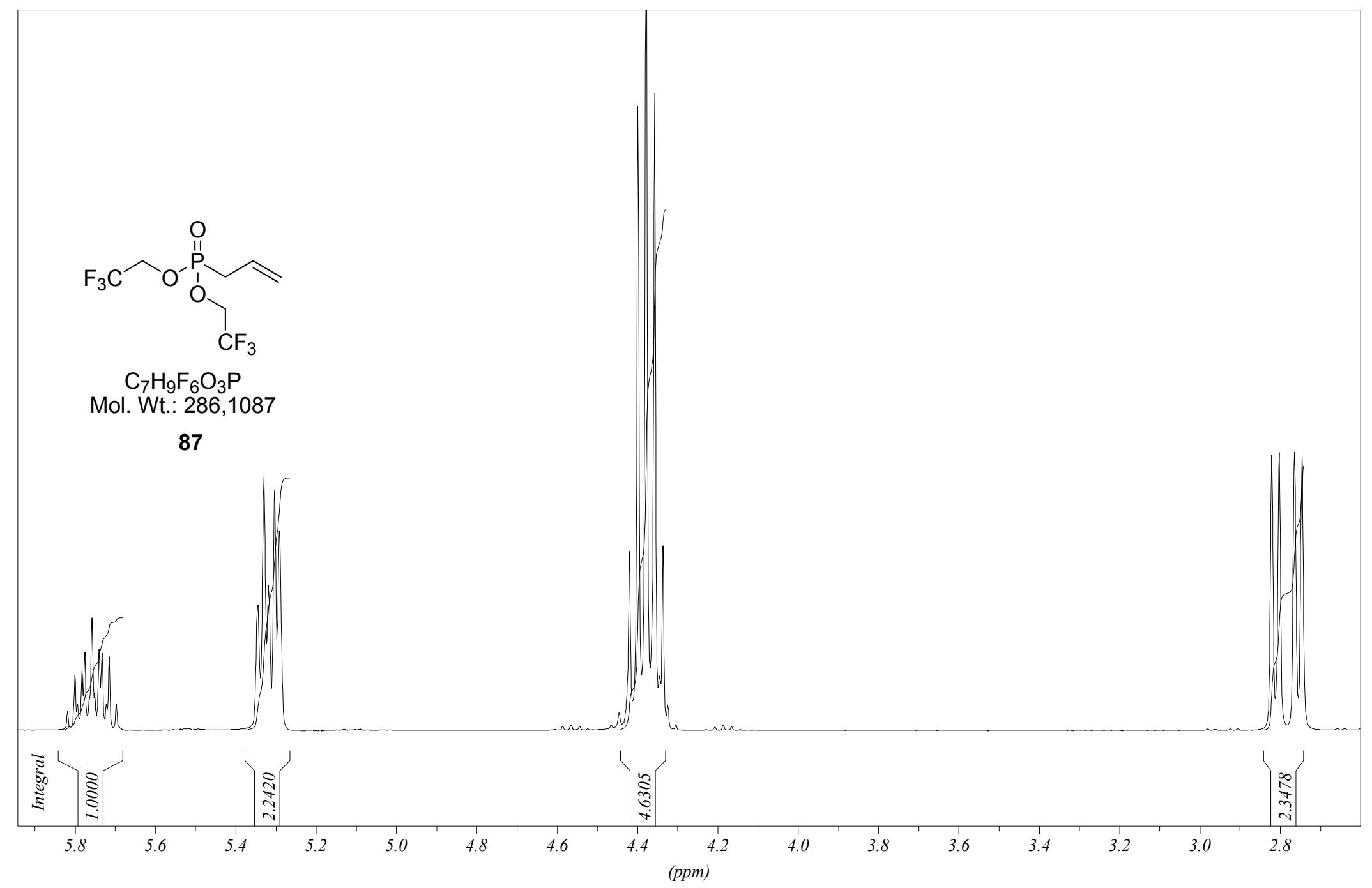




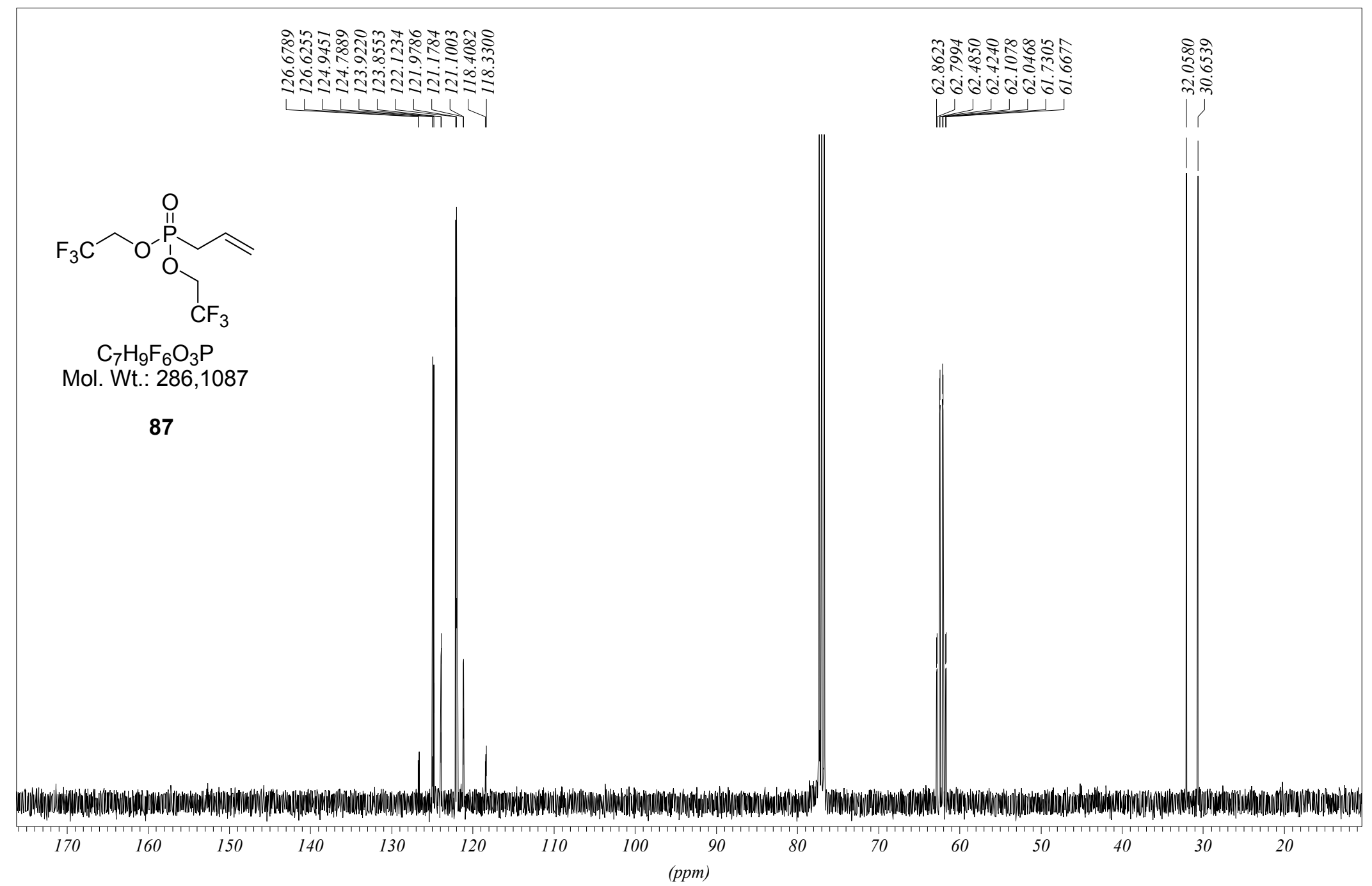




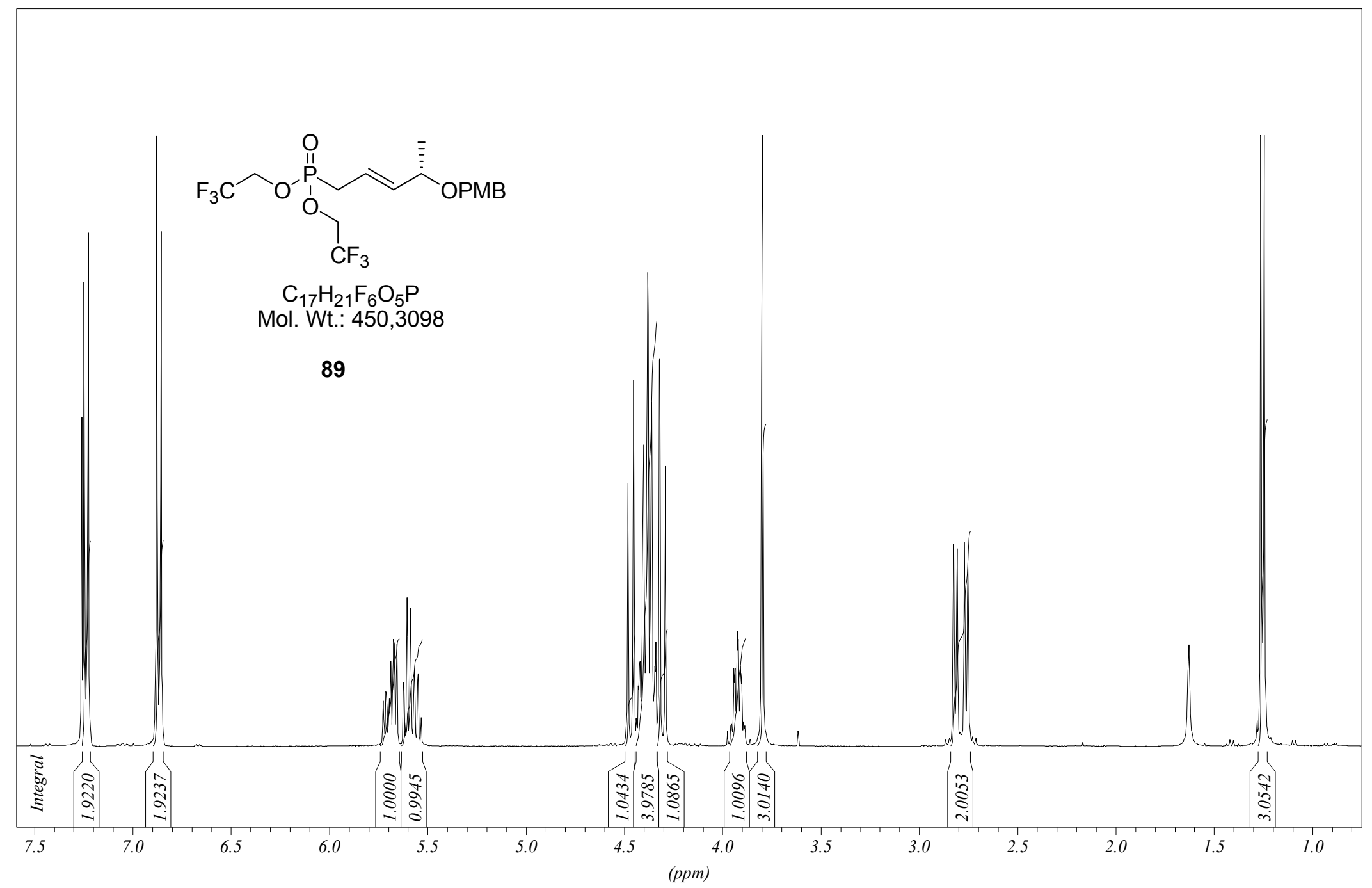




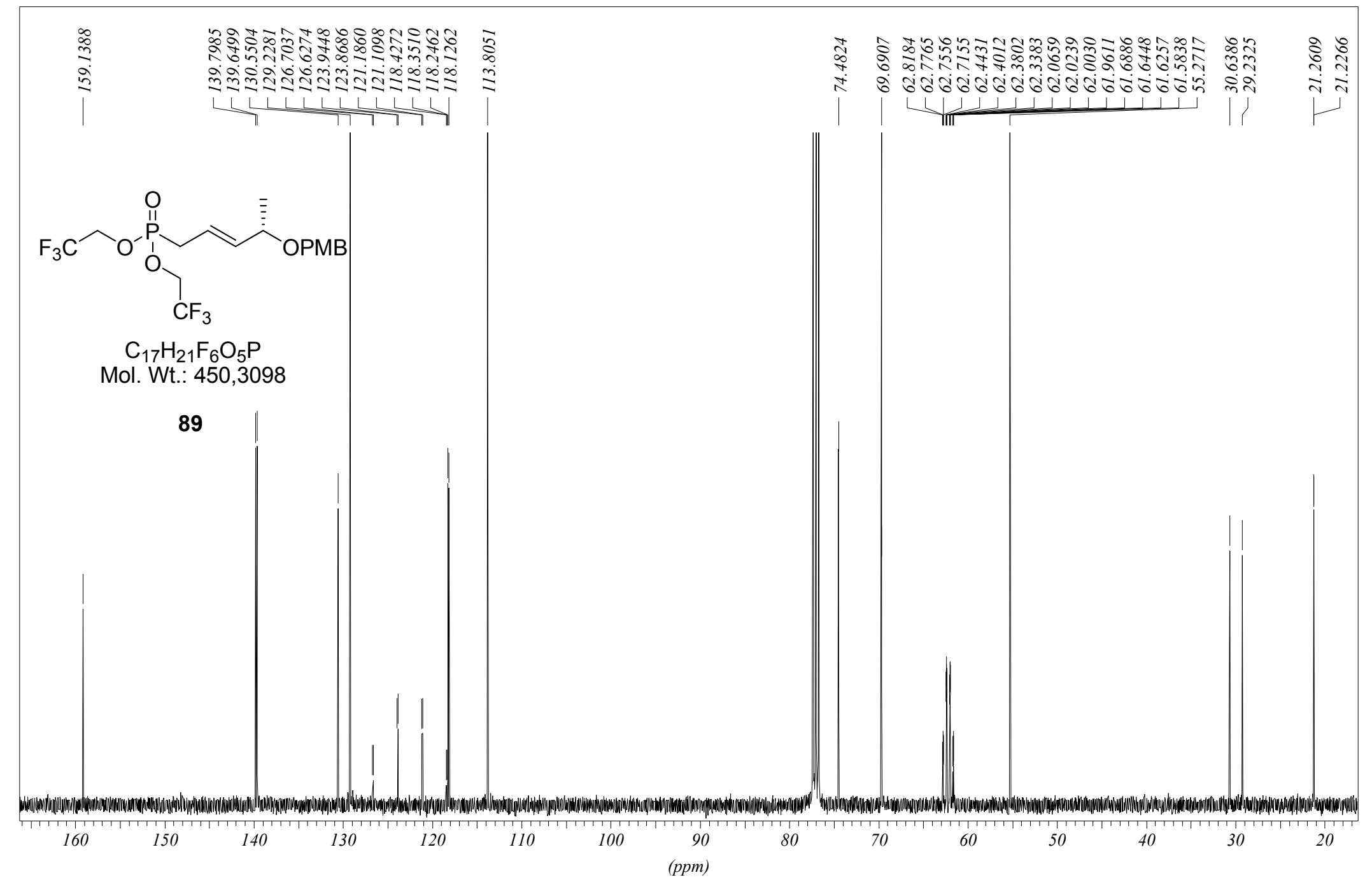




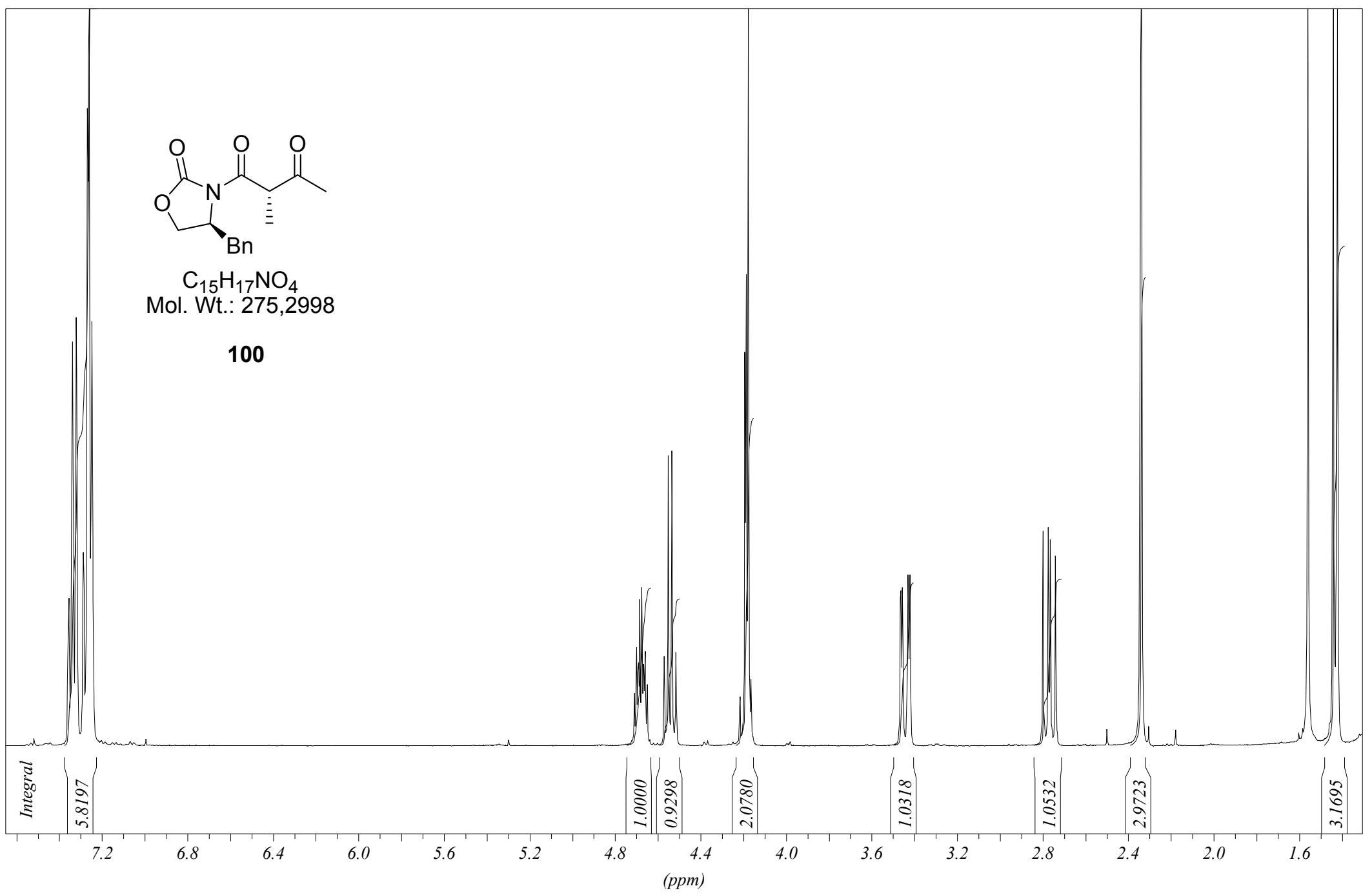




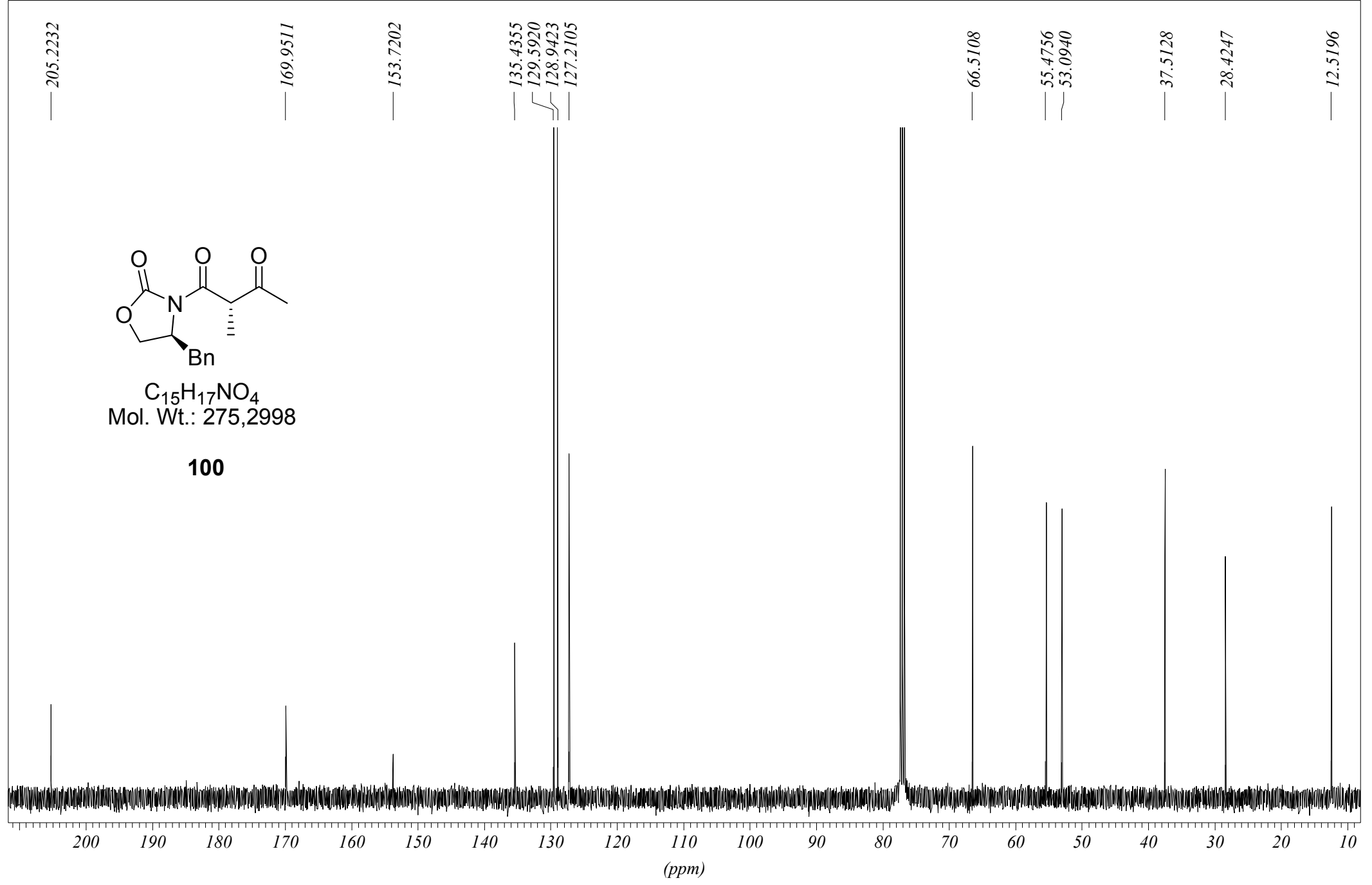




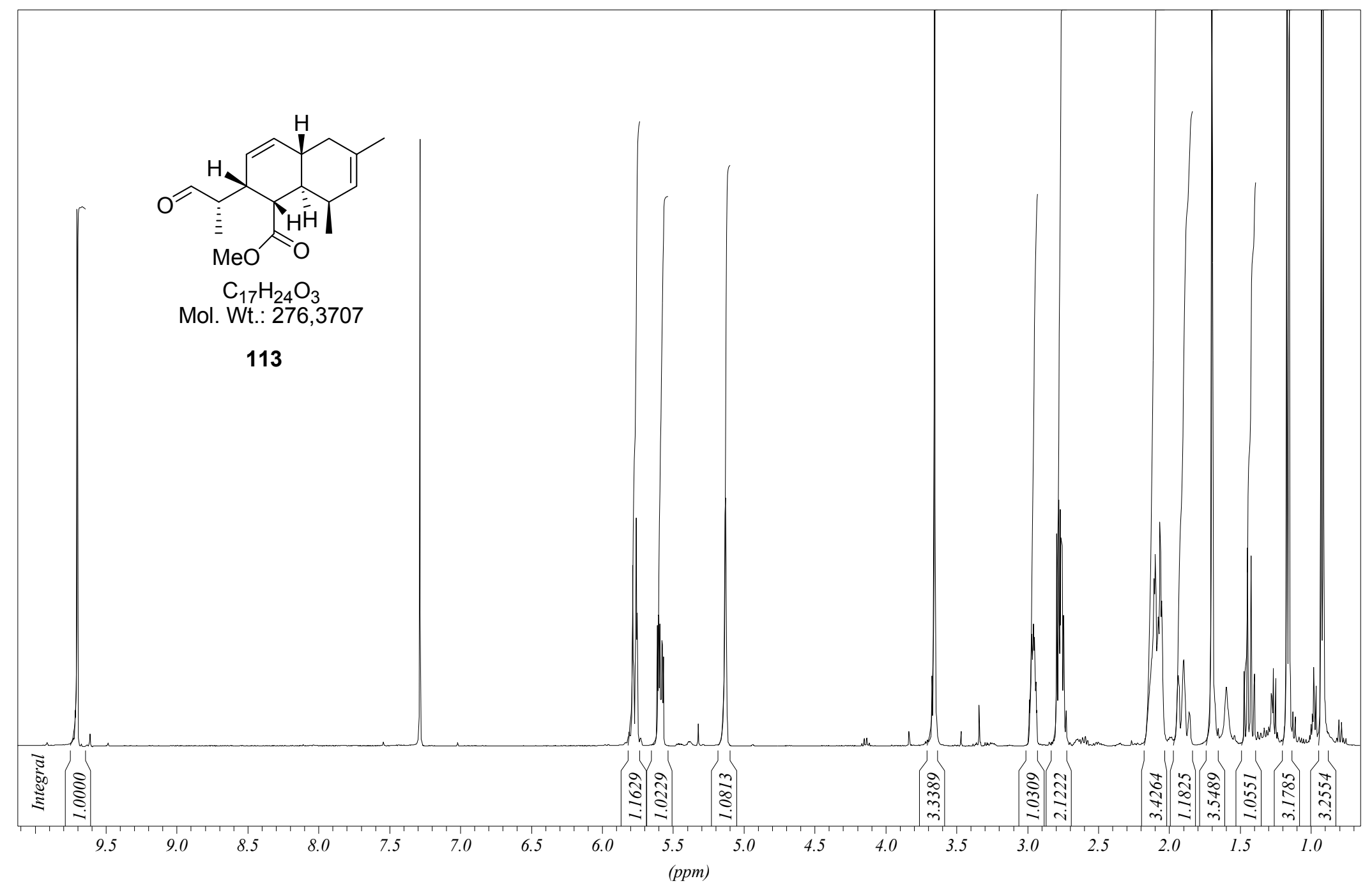




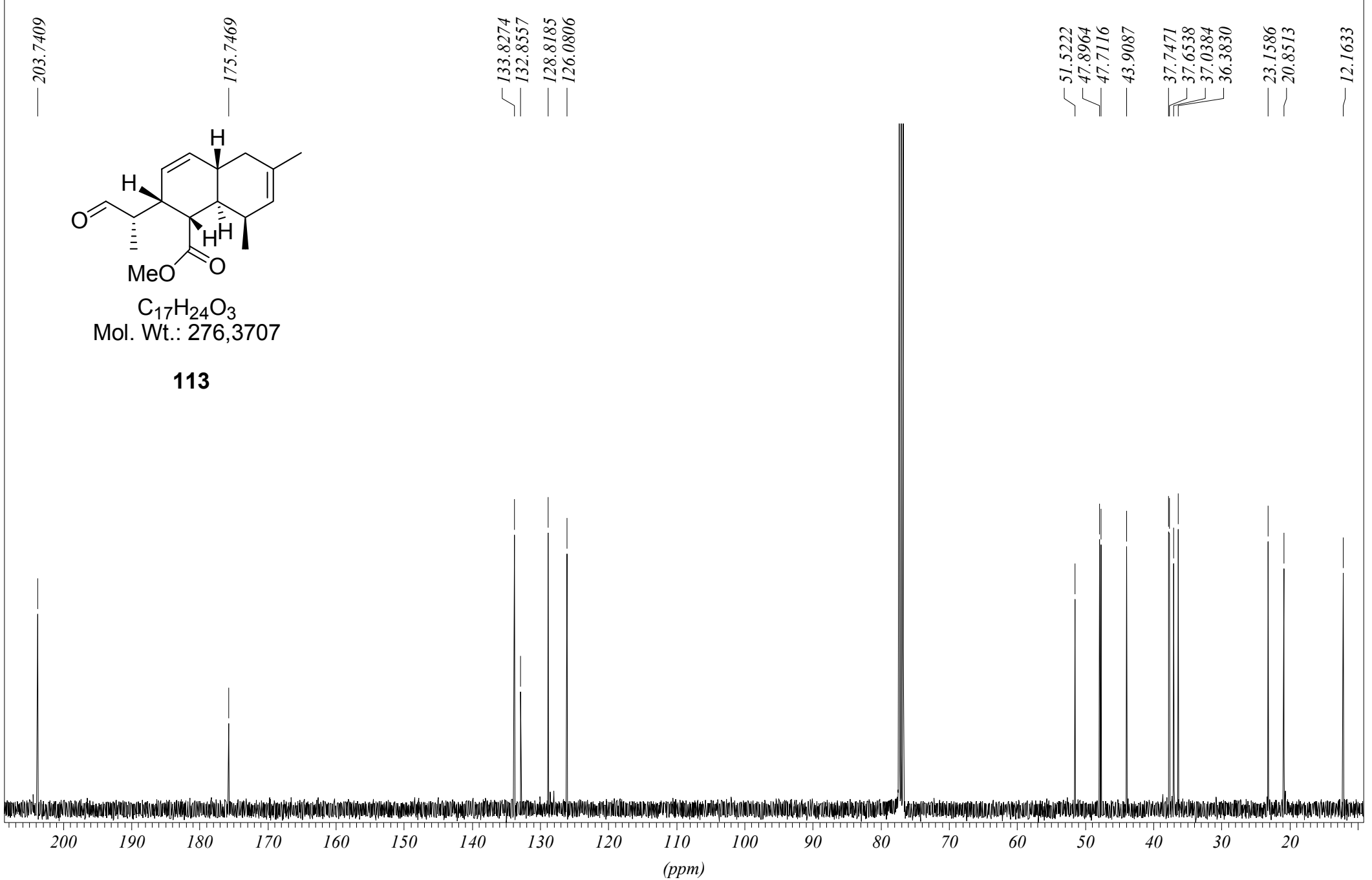




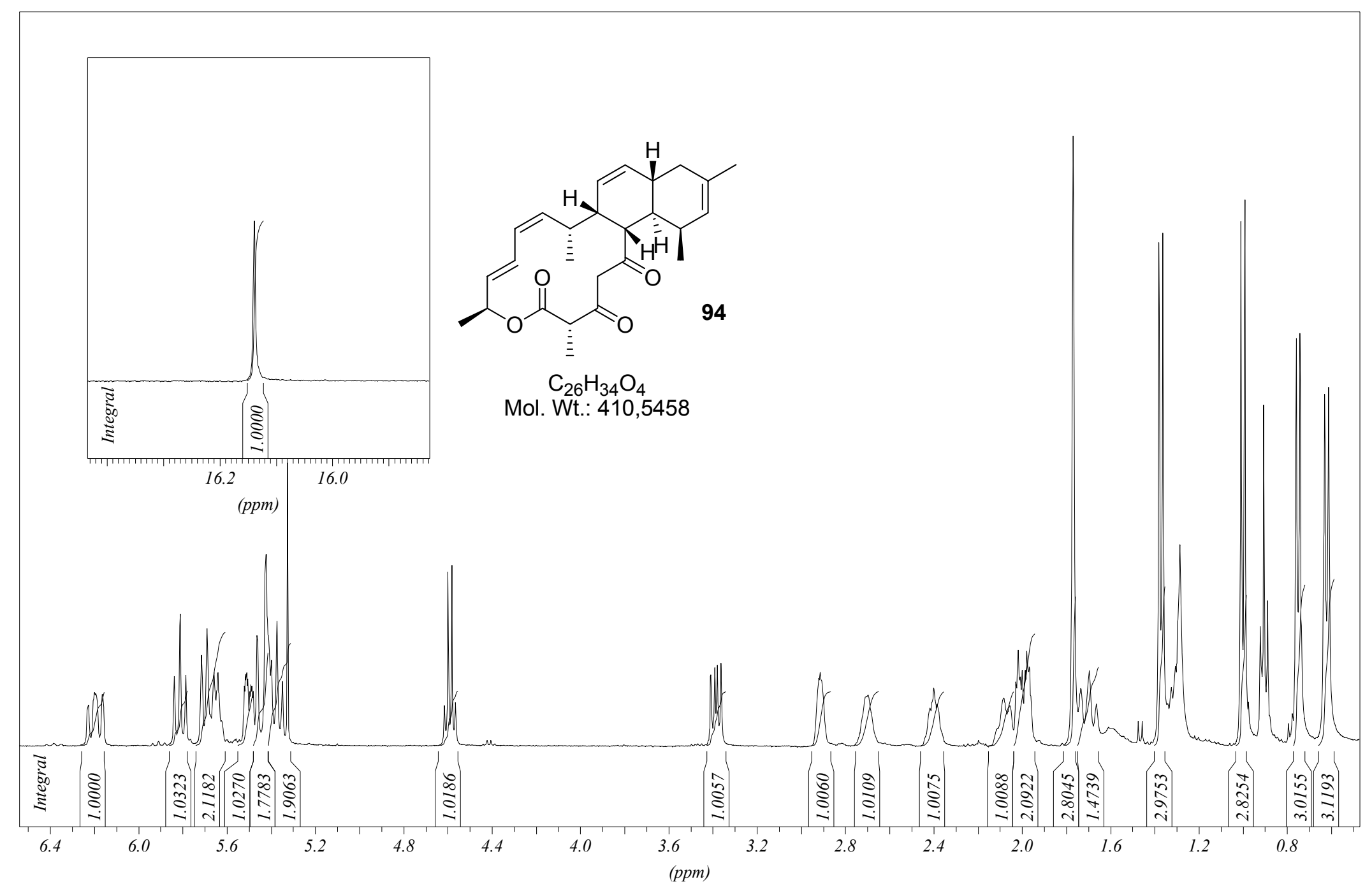




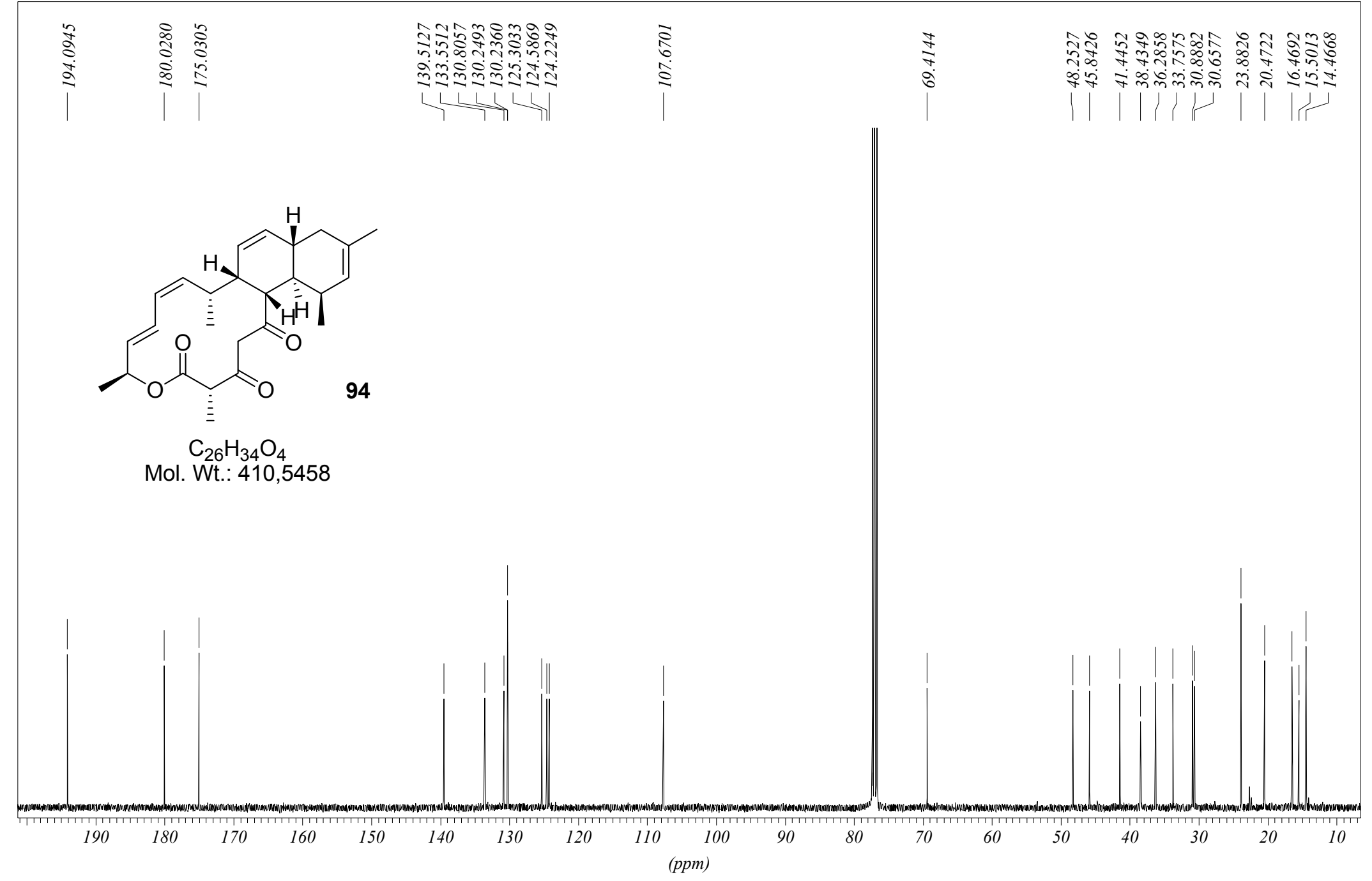




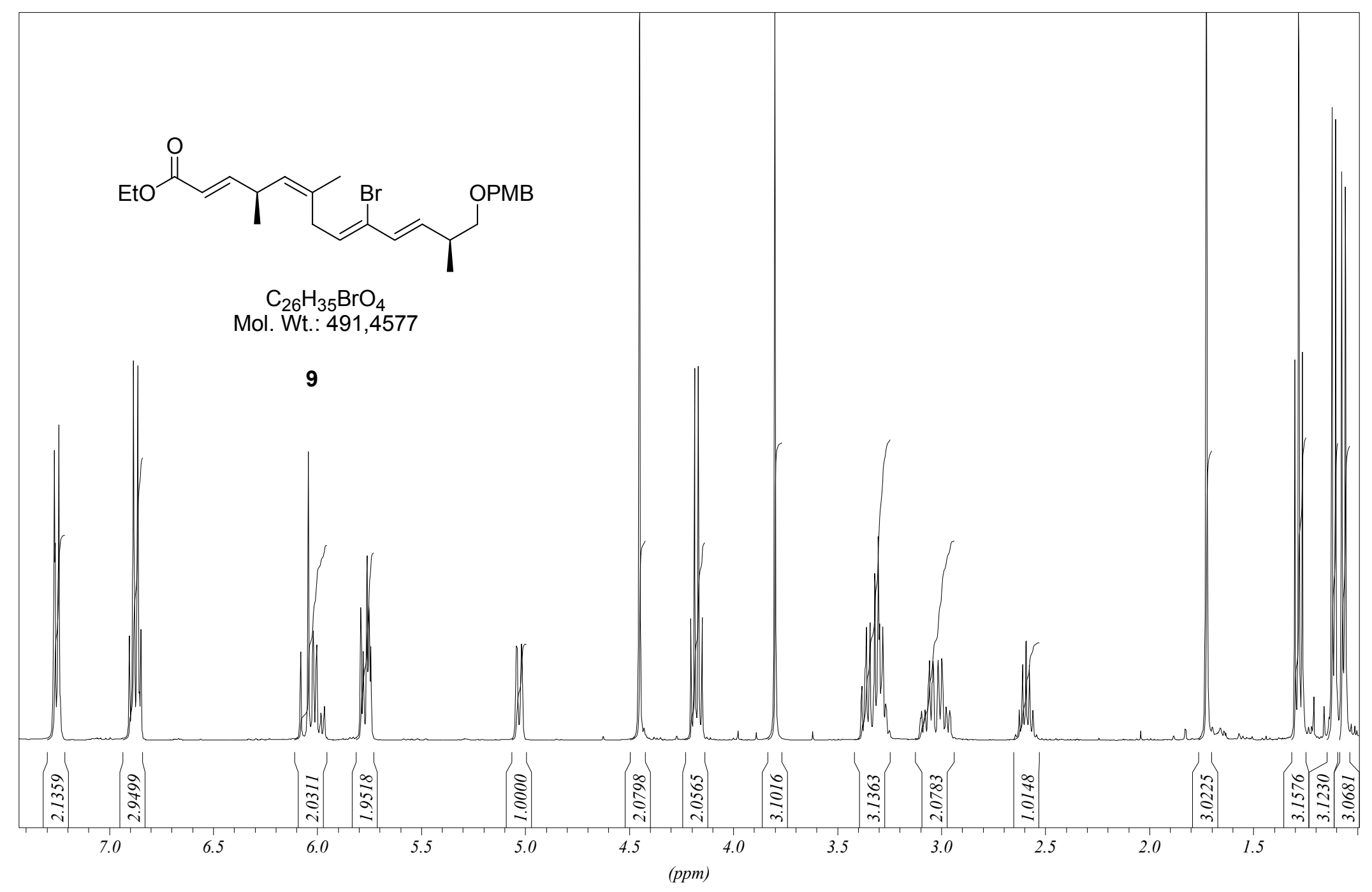




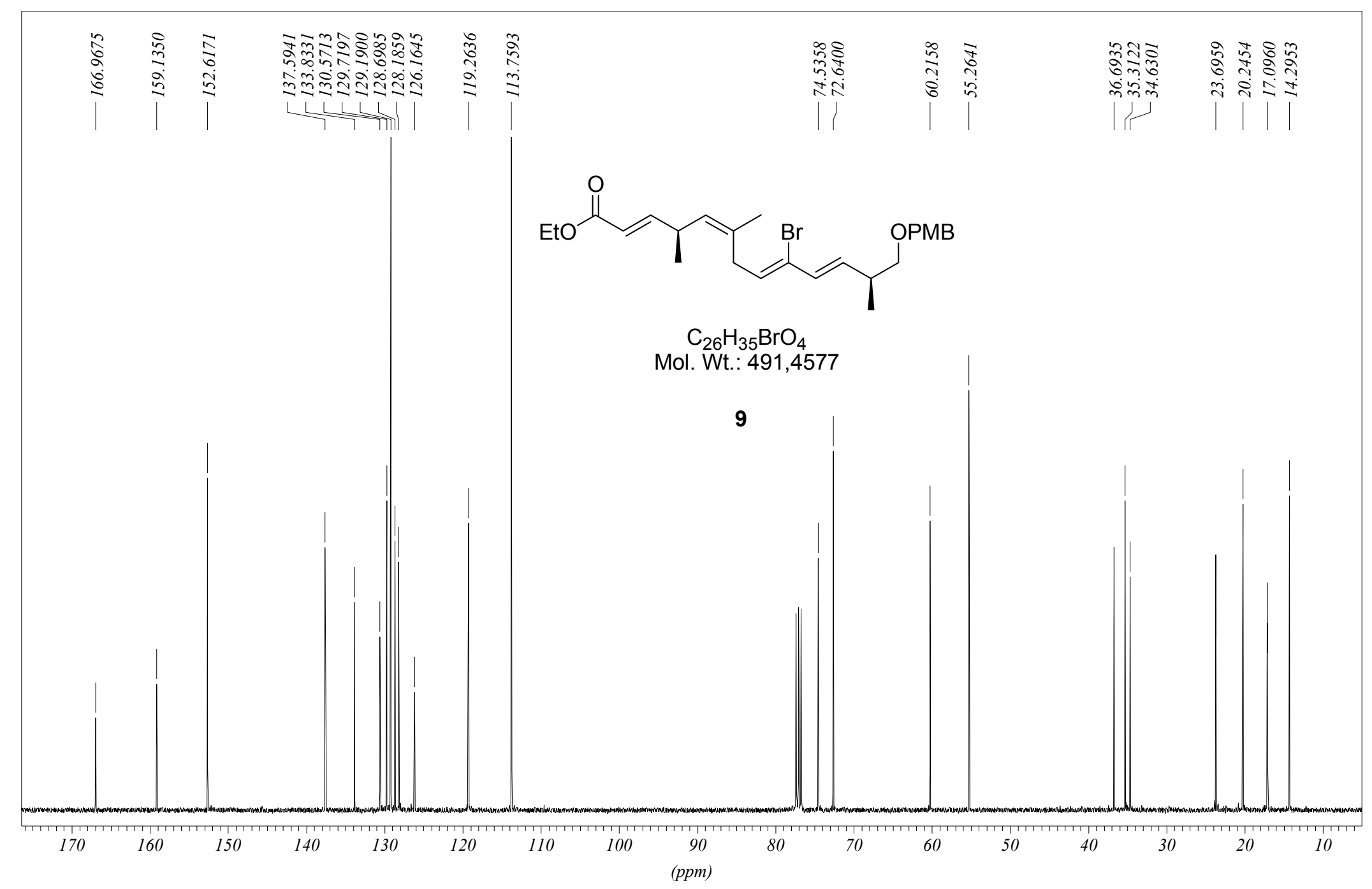




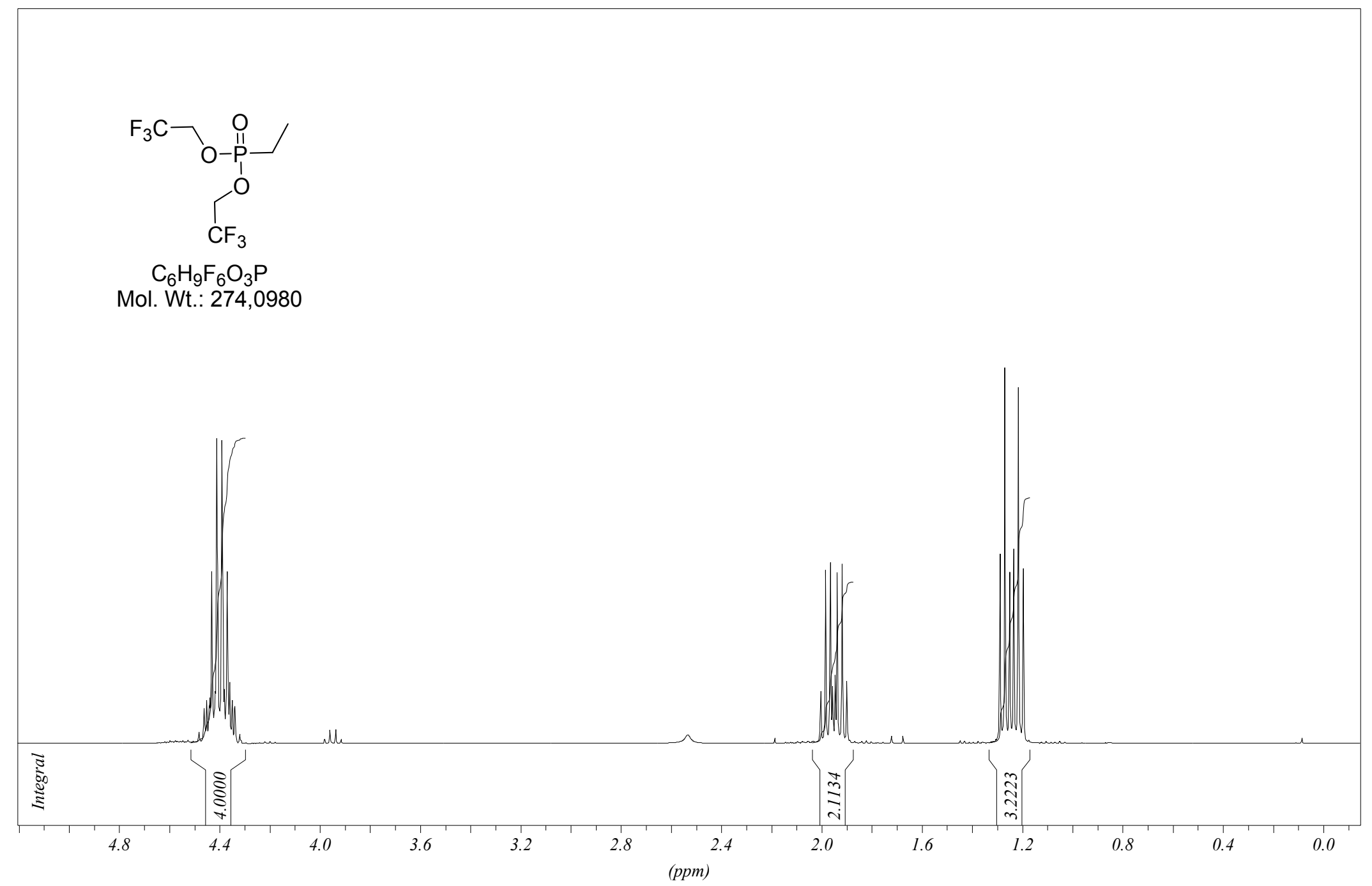




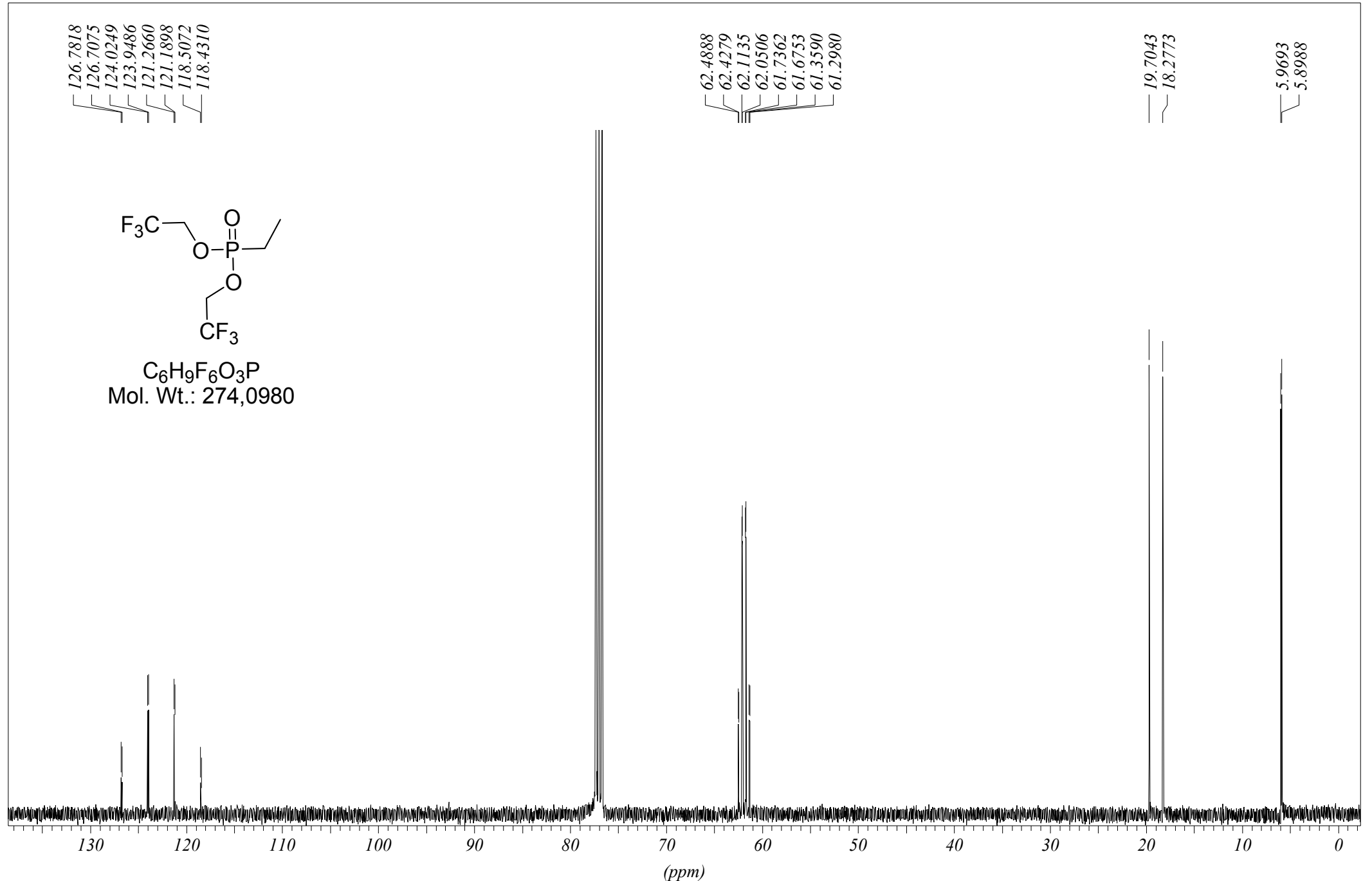




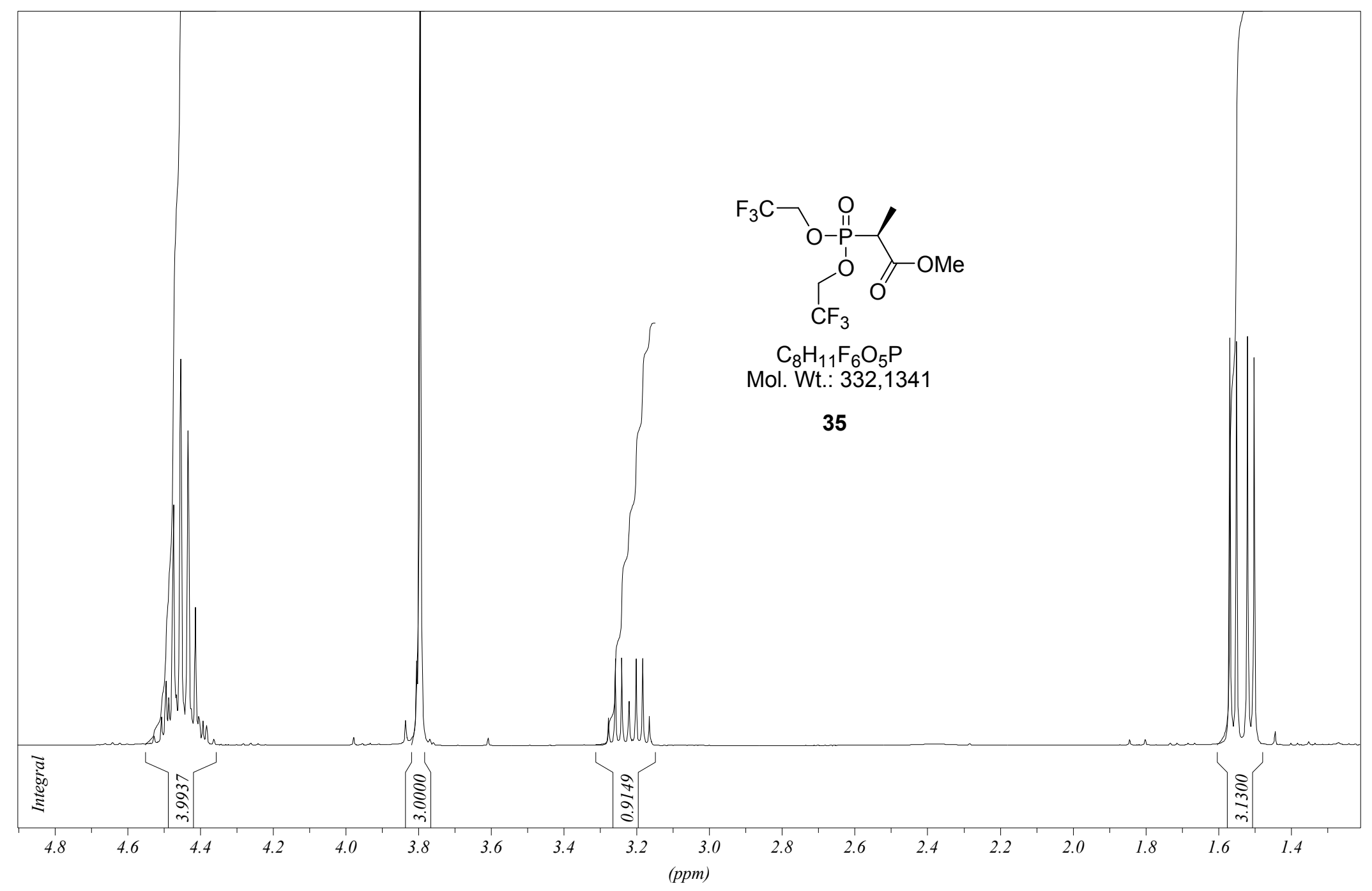




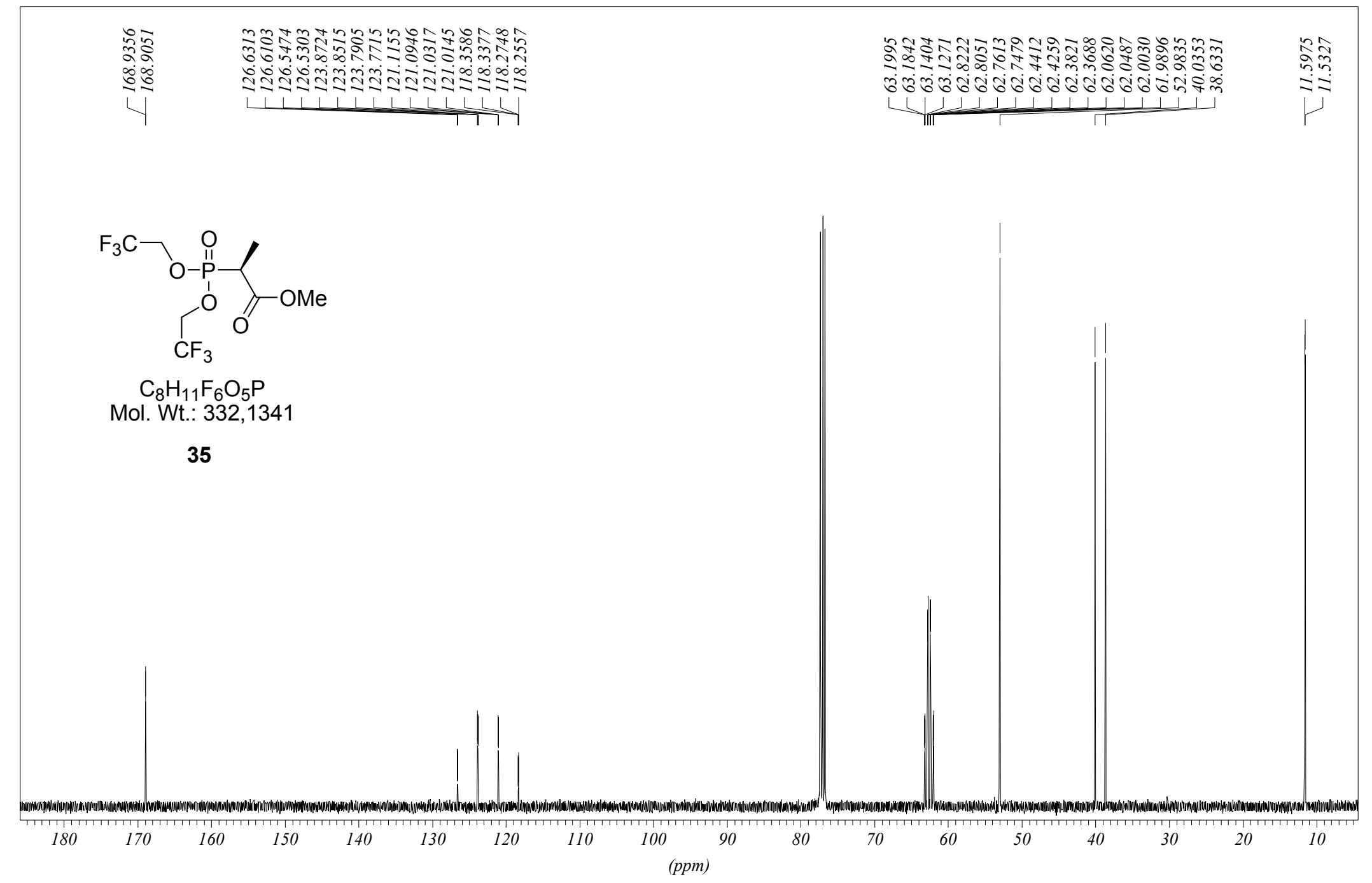




\section{Lebenslauf}

Nicola Rahn (geb. Pögel)

\section{Persönliche Daten}

Geburtsdatum: $\quad$ 10.08.1979 in Hildesheim

Staatsangehörigkeit: deutsch

Familienstand: verheiratet

\section{Ausbildung}

1986 - 1990 Grundschule in Harsum

1990 - 1999 Gymnasium Marienschule in Hildesheim

$1999 \quad$ Abitur

1999 - 2004 Chemiestudium an der Leibniz Universität Hannover

2001 - 2002 Halbjähriges Praktikum bei Prof. K.P.C. Vollhardt an der UC Berkeley

2004 Diplom in Chemie

Titel der Diplomarbeit: „Synthese von Polyketidfragmenten durch

Epoxidumlagerung“ (Prof. Dr. Markus Kalesse)

2004 - 2007 Promotion unter der Leitung von Prof. Dr. Markus Kalesse

Titel der Doktorarbeit: „Die Totalsynthese von Chlorotonil A“ 